\title{
DEVELOPMENT OF A WEB-BASED COLLABORATIVE PPGIS TO SUPPORT MUNICIPAL PLANNING
}

By

\section{Muhammad Atif Butt}

\author{
Master of Applied Science, Ryerson University, Toronto, Canada 2007 \\ Master of Computer Science, Hamdard University, Pakistan 2001 \\ Master of Information Technology, Grace College, Pakistan 1999 \\ Master of Business Education, The Punjab University, Pakistan 1998
}

\author{
A thesis \\ presented to Ryerson University \\ in partial fulfilment of the \\ requirements for the degree of \\ Doctor of Philosophy \\ in the Program of \\ Civil Engineering
}

Toronto, Ontario, Canada, 2015

(C) Muhammad Atif Butt 2015 


\section{Author's Declaration}

I hereby declare that I am the sole author of this dissertation. This is a true copy of the dissertation, including any required final revisions, as accepted by my examiners.

I authorize Ryerson University to lend this dissertation to other institutions or individuals for the purpose of scholarly research. I further authorize Ryerson University to reproduce this dissertation by photocopying or by other means, in total or in part, at the request of other institutions or individuals for the purpose of scholarly research.

I understand that my dissertation may be made electronically available to the public. 


\title{
Development of a Web-based Collaborative PPGIS to Support Municipal Planning
}

\author{
Muhammad Atif Butt \\ Doctor of Philosophy, 2015, Civil Engineering, Ryerson University
}

\begin{abstract}
The use of computing technologies to support workflows related to the planning and development of a municipality dates back to the late 1950s. The main boosts of computing technologies and applications, Geographic Information System (GIS) and Geographic Information Technology (GIT), in relation to a planning and decision-making context, became evident when the use of Public Participation GIS (PPGIS) started in the 1980s.

Collaboration is an important part of many tasks involving people from different organizations, in which maps often play a central role in informing and improving debates and facilitating the decision-making process. It allows diverse stakeholders to share and view maps or spatial images interactively over the Web in real-time, among other platforms. The geospatial collaboration technology not only provides an effective solution to decision makers, but also facilitates scientific and public debates with real-time geospatial information. More recently, some efforts have been made using open map services to develop simple map-sharing applications. However, little has been done on designing and developing such online open source tools in the context of municipal planning and management. Further, a literature review indicates the lack of scientific publications on empirical studies of their practical applications.
\end{abstract}

Web-based PPGIS applications, among others, have now been widely recognized as an efficient and integral part of sound planning and development processes to support public participation. However, GIS alone cannot make the planning process more participative. Demands for Web-based PPGIS tools, integrated with other information and computer-supported cooperative work (CSCW) tools, have rapidly become increasingly important for supporting collaborative participation during a decision-making process. Therefore, the establishment of public participation in GIS-based applications is an optimistic step taken by the researchers that are progressively working on municipal planning projects that incorporate public participation.

The main aim of this research is to provide a Collaborative PPGIS (Co-PPGIS) to enhance public participation in municipal planning related workflows. A research prototype has been developed and its usability is evaluated by adopting the evaluation criteria for the research prototype, as only proper testing will demonstrate whether the prototype is usable or the Co-PPGIS design framework is successful in meeting end-users' requirements. 


\section{Acknowledgements}

First of all, I would like to express my great appreciation and thanks to my supervisor, Dr. Songnian Li, for his valuable advice and ideas as well as constructive suggestions and productive comments on my thesis. I also thank him for providing me the great opportunity to continue my study in the field of GIS and to work with him as a research assistant during my years at Ryerson University. I greatly appreciate his efforts to provide me financial supports throughout the course and my thesis studies. Without his support, I would never have completed this thesis work.

I would like to express my great admiration and gratitude to the following people who gave me a great helping hand in the completion of this PHD research:

Dr. Ahmed Shaker, Dr. Michael A. Chapman and other faculty and staff members in the Department of Civil Engineering, for their academic and technical help and support. Dr. Wanhong Yang, the external examiner, for his insightful inquiries and constructive comments.

Rachel Harpley, Kim Kritzer and Dianne Mendonca for their administrative support.

Shahram Sattar, Wei Huang and Wai Yeung Yan, other graduate students in the program of Civil Engineering at Ryerson University, for their help. The study time with them always recalls my beautiful and happy recollections.

Last, but not least, I wish to express my thankfulness to my late parents and to my wife and sons for their unconditional love and support with patience, and encouragement. Without their help and understanding, I would never have completed this thesis. 


\section{Table of Contents}

Author's Declaration ___

Abstract____

Acknowledgements ___ iv

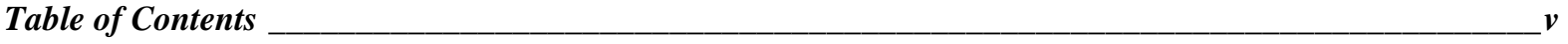

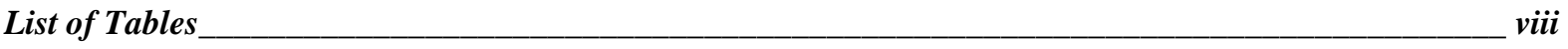

List of Figures_______ ix

List of Abbreviations and Acronyms ____

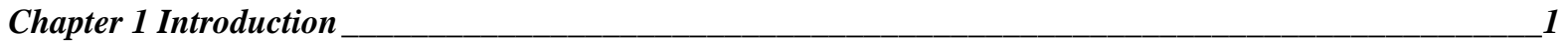

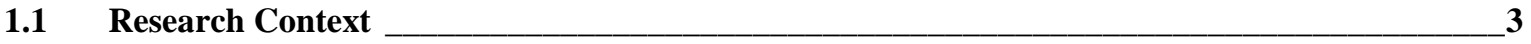

1.1.1 Central Idea ___ 3

1.1.2 Issues and Concerns in Existing Participation Practices and PPGIS ___

1.1.3 Web-based Real-time Co-PPGIS as a Potential Solution___

1.2 Research Objectives ___ 9

1.3 Overview of Research Methodology ___ 10

1.3.1 Review of Literature, PPGIS Frameworks and Technologies ___ 11

1.3.2 Requirements and Design Modeling of Co-PPGIS ___ 11

1.3.3 Prototyping_____ 12

1.3.4 Prototype Evaluation ___ 12

1.4 Organization of the Thesis ___

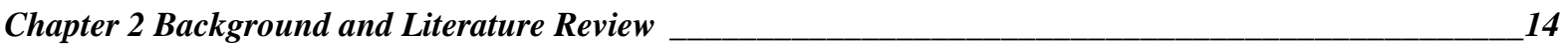

2.1 Concepts of Public Participation in Planning and Management ___ 14

2.1.1 Concepts of Public Participation ___

2.1.2 Degrees of Public Participation ___ 15

2.1.3 Existing Public Participation Practice during Municipal Planning and Development ___ 19

2.2 Public Participation GIS (PPGIS) ___ 21

2.2.1 The Shift to Electronic Participation ___ 21

2.2.2 Web-based Public Participation GIS ___ 22

2.2.3 Role of CSCW and Groupware in PPGIS ___ 23

2.3 Examination of Selected Web-based Participatory GIS _ 25

2.3.1 Review of Selected Collaborative Participatory GIS ___ 27

2.3.2 Comparison of the Selected Online Collaborative Participatory GIS Applications___ 34

2.3.3 Summary of Closely-related Research Models ___ 39

2.4 Review of Usability Evaluation for Web-PPGIS ___ 44

2.5 Enabling Technologies Used in Web-PPGIS ____ 46

2.6 Discussion ___ 51

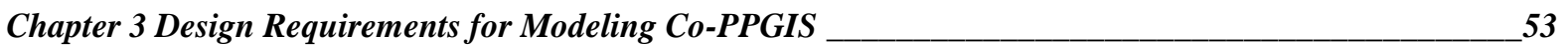

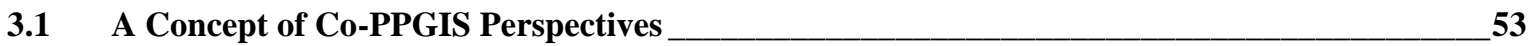

3.2 Co-PPGIS Conceptual Framework__ 57

3.3 Understanding a Co-PPGIS Meeting Infrastructure____ 61

3.4 Gaps in Existing Municipal Planning Practices and Possible Improvements using Co-PPGIS_65 
3.5 Identify Direct Relationship between Arnstein's (1969) Ladder of Participation and Co-PPGIS

3.6 Identify Direct Relationship between Weidemann and Fermers (1993) Ladder of Participation and Co-PPGIS Components 68

3.7 Methodology Adopted for Requirements Engineering of Co-PPGIS__ 70

3.7.1 RE Process of Co-PPGIS _ 70

3.7.2 Co-PPGIS Context Model _ 73

3.7.3 Functional Requirements of Co-PPGIS ___ 77

3.8 Co-PPGIS High-level Architecture and Flow Models ___ 80

3.9 Summary _ 85

Chapter 4 Prototype System Development____ 86

4.1 Overview of Co-PPGIS Prototype Application Development___ 86

4.2 Co-PPGIS Synchronous Participatory Environment (CSPE) High-level Architecture ___ 87

4.3 Co-PPGIS Asynchronous Participatory Environment (CAPE) High-level Architecture __ 90

4.4 Co-PPGIS Component Service-oriented Architectures___ 91

4.5 Prototype Implementation _ 102

4.5.1 Co-PPGIS Asynchronous Participatory Environment Blog Component ___ 103

4.5.2 Co-PPGIS Synchronous Participatory Environment (CSPE)__ 106

4.5.3 Co-PPGIS: Collaborative Map-sharing Component ___ 108

4.5.4 Walkthrough of Co-PPGIS Map-sharing Component Services___ 111

4.6 Demonstration of the Prototype using Mock-up Scenarios - Co-PPGIS Implementation in Planning and Management related Activities__ 122

4.7 Discussion _ 127

4.7.1 Intervening Problems and Issues __ 127

4.7.2 Technological Issues ___ 128

4.8 Summary _ 131

Chapter 5 Usability Evaluation of Co-PPGIS using Mock Case Study ___ 132

5.1 Proposed Usability Evaluation Methodology for Co-PPGIS _ 132

5.2 Generating Usability Matrix ___ 136

5.3 Case Study: Evaluating the Usability of Co-PPGIS Map-sharing System - As a Participatory

Municipal Planning Support Tool___ 137

5.3.1 Selected Co-PPGIS Prototype Web Interfaces for Scenario-based Testing ___ 138

5.3.2 Usability Evaluation Criteria of Co-PPGIS _ 139

5.3.3 Usability Evaluation Methodology for Co-PPGIS Map-sharing Interface___ 140

5.3.4 Summary __ 153

Chapter 6 Conclusions and Recommendations____ 154

6.1 Realization of Research Objectives ___ 154

6.2 Discussion of Thesis Outcomes __ 156

6.3 Contribution and Significance___ 158

6.4 Recommendation for Further Studies___ 160

References____ 162

APPENDIX 1 Participant's Basic Information Pre-Test Questionnaire _ 170

APPENDIX 2 Feedback Questionnaire (Post-Test Questionnaire) ___ 171 
APPENDIX 3 Tabular Display of Results Depicting User Profile \& Performance

APPENDIX 4 Spearman Rank Correlation

APPENDIX 5 Two-Way ANOVA

177

APPENDIX 6 Sequence Diagrams of Co-PPGIS Services

APPENDIX 7 Usability Aspects to Measure Social Adoptability of Co-PPGIS

APPENDIX 8 List of Selected Application for Evaluation 


\section{List of Tables}

Table 2.1: Issues and Concerns in Existing Practice of Public Participation...........................................................20

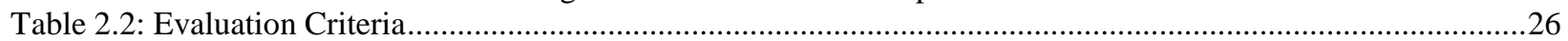

Table 2.3: Comparison of Selected PPGIS Applications (Butt and Li 2014) .........................................................35

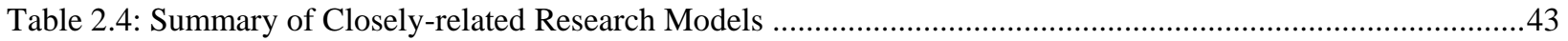

Table 5.1: Specification of Usability Measuring Criteria Matrix ..........................................................................136

Table 5.2: Spearman Correlation between Usability Indicators and User Characteristics.......................................149

Table 5.3: Spearman Rank Correlation between User Characteristics and Contentment Indicators .........................151

Table 5.4: Tukey HSD - Average Clicks to Perform a Single Task (All Groups) ......................................................152

Table 5.5: Tukey HSD - Average Clicks to Perform a Single Task (GIS vs. Other Groups) ....................................153 


\section{List of Figures}

Figure 1.1: Issues and Concerns in Existing Practices of Public Participation ..........................................................5

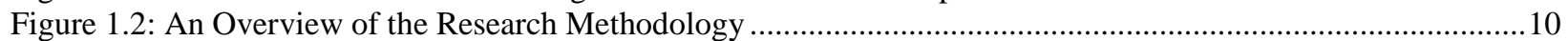

Figure 2.1: Modified Conceptual Diagram of E-government Framework (McClure 2000) ........................................15

Figure 2.2: Modified Ladder of Public Participation (Arnstein 1969) .....................................................................16

Figure 2.3: Modified Public Participation Ladder (Wiedemann and Fermers 1993) ................................................17

Figure 2.4: Modified Five Reasons of Participation by CIAC (http://www.oregon.gov) ..........................................18

Figure 2.5: Traditional and Web-based Participatory Processes (Butt and Li 2014) ...............................................22

Figure 2.6: Virtual Slaithwaite Project (last accessed in January 2014: http://www.ccg.leeds.ac.uk/slaithwaite/) .....27

Figure 2.7: Landscape Plan Königslutter am Elm (last accessed in January 2014: http://www.koenigslutter.de/) .....29

Figure 2.8: Argumentation Maps (last accessed URL: http://www.agile-

online.org/Conference_Paper/CDs/agile_2005/papers/66_Carsten\%20Kessler.pdf)........................................31

Figure 2.9: GIS-enabled Online Forum (http://www.i-cherubini.it/mauro/blog/2007/06/26/) ................................32

Figure 2.10: GIS-enabled Online MapChat (URL: http://www.mapchat.ca) ...........................................................

Figure 2.11: Modified Argumentation Map Model (Rinner 2006) .....................................................................40

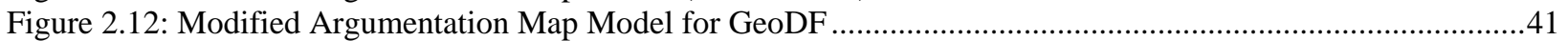

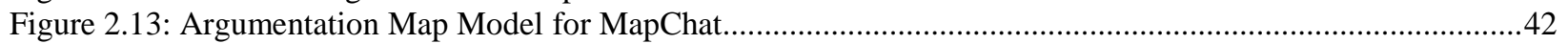

Figure 2.14: Modified Categories of Free and Non-Free Software (http://www.gnu.org/philosophy/categories.html)

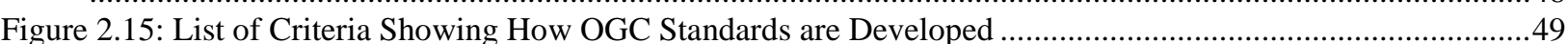

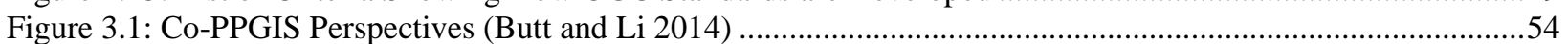

Figure 3.2: Co-PPGIS Workflow Processes and Service Abilities (Butt and Li 2014) ............................................56

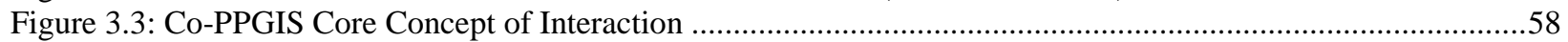

Figure 3.4: Co-PPGIS Goals with respect to Core Elements of Interactions (Butt and Li 2014) .............................59

Figure 3.5: Stages of Theoretical Framework of Co-PPGIS ............................................................................60

Figure 3.6: Public Meeting Scenario at Three Stages/ levels of Interaction ...........................................................61

Figure 3.7: Co-PPGIS Synchronous and Asynchronous Concept for Planning and Management (Butt and Li 2012)62

Figure 3.8: Logical Perspective in Relation to Municipal Planning using Co-PPGIS (Butt and Li 2012) ..................64

Figure 3.9: Identifying Relation between Existing Participation Practices and the Suggested Co-PPGIS ..................65

Figure 3.10: Modified Arnstein's Ladder Applied to Co-PPGIS Components (Butt and Li 2014)...........................67

Figure 3.11: Wiedemann and Fermers Ladder (1993) Applied to Co-PPGIS Components-pyramid which enables the

Exchanging of Information at Different Participation Level among Public and Decision-maker.....................69

Figure 3.12: Workflow of the Requirements Engineering Process for Co-PPGIS ................................................72

Figure 3.13: Co-PPGIS Actors (Stakeholder) Onion Model Rings Show Stakeholders' Ability to Influence the

System in Terms of Their Level of Participation and/or Decision-making Processes .......................................75

Figure 3.14: Context Diagram of the Co-PPGIS (Butt and Li 2012)................................................................76

Figure 3.15: List of Functional Requirements for Participants ..........................................................................

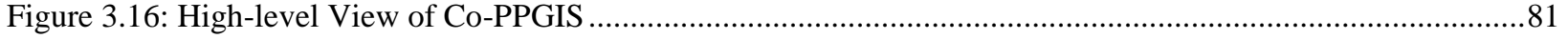

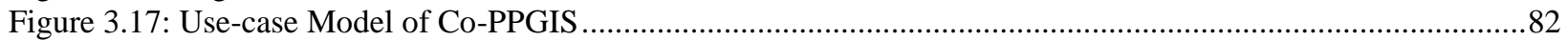

Figure 3.18: Co-PPGIS Main Functional Processes in the Form of Data Flow Model ..............................................83

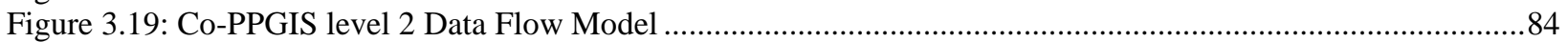

Figure 4.1: High-level Architecture of Co-PPGIS Synchronous Participatory Environment.....................................89

Figure 4.2: Logical Architecture of GIS-based Discussion Forum....................................................................90

Figure 4.3: Flow Diagram of Co-PPGIS Component Services.................................................................................91

Figure 4.4: Information Flow Model of Login Management Service ..................................................................93

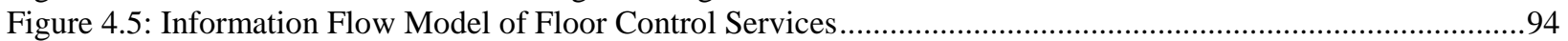

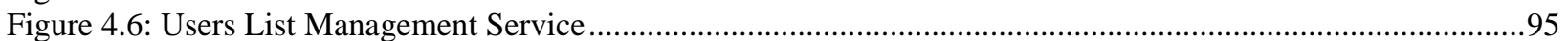

Figure 4.7: Information Flow Model of Map-sharing Service............................................................................95

Figure 4.8: Android-based Geo-Tagging of Incidents on a Shared View of Map …….............................................96

Figure 4.9: General Chat Service using BlazeDS and Flex ……........................................................................97

Figure 4.10: Map Feature-based Chatting against Marked Incidents on a Shared View of Map ..............................97

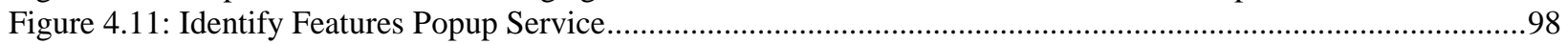

Figure 4.12: Geometry Shapes Geo-tagging of Incidents on a Shared View of Map .................................................99

Figure 4.13: Multimedia Geo-tagging Related to Incidents during Map-sharing Environment ...............................100 
Figure 4.14: Bookmark Service for Saving Map Extent with Annotation .....

Figure 4.16: Spatial Data of York Region (Town Markham Amendment Plan) is Overlaid on the Google Map (can be accessed at http://coppgis.com/wolpgis-f/?p=54).....

Figure 4.17: Co-PPGIS GIS-based Discussion Forum Component (Online access URL: http://coppgis.com/prism)

Figure 4.18: CSPE Interface with Real Public Meeting Document Sharing and Basic Map-sharing Streams (Online access URL: http://coppgis.com:8080) ..........................................................................................107

Figure 4.19: Co-PPGIS Map-sharing Component (online access at http://coppgis.com/geomeet4) .......................109

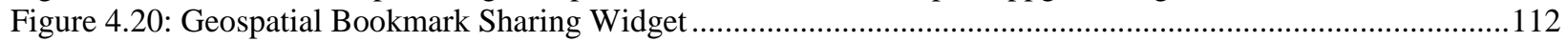

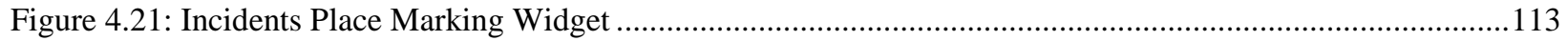

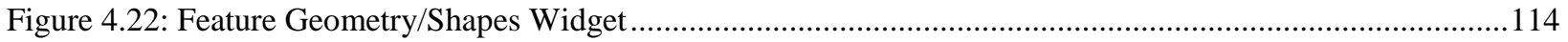

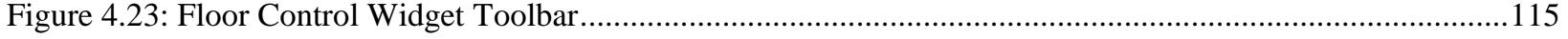

Figure 4.24: Show/ Hide Pointer Tools of Floor Control Widget....................................................................115

Figure 4.25: Deactivate and Activate Tool of Floor Control Moderation Widget ..................................................116

Figure 4.26: Send Request Tool and Activate Request Tool of Floor Control Widget ........................................117

Figure 4.27: Feature-based Multimedia Adding Widget .......................................................................... 118

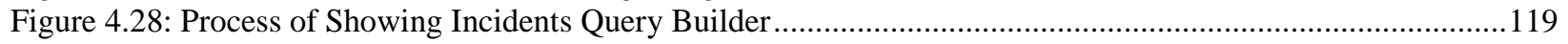

Figure 4.29: Process of Showing Incidents Report Builder ..................................................................120

Figure 4.30: Process of Enabling Video Camera Popup during Co-PPGIS Map-sharing Participatory Environment

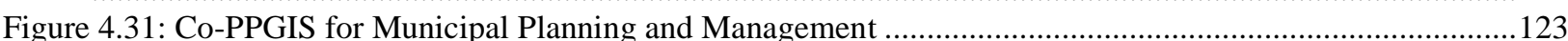

Figure 4.32: Co-PPGIS for Preparing Master/Land use Plan through Collaborative Approach............................124

Figure 4.33: Co-PPGIS for Suitable Park Area Identification ................................................................126

Figure 5.1: Usability Evaluation Flow Diagram for the Assessment of a Co-PPGIS Prototype Application............132

Figure 5.2: Hard Copy Map (on Left) and Google Map (on Right) (Source of Hard Copy Map: http://www.york.ca)

Figure 5.3: Co-PPGIS Map-sharing Interface Overlaid the York Region Proposed Amendment Plan (ROPA 2) in the City of Vaughan. Web URL to Access Synchronous Co-PPGIS Map-sharing Web Interface:

http://coppgis.com/geomeet1

Figure 5.4: Spatial Data of York Region (Town Markham Amendment Plan) Overlaid on the Google Map (Base Layer) Using Co-PPGIS Component Discussed in Section 4.2.1, URL to Access CAPE Blog: http://coppgis.com/wolpgis-f/?p=42

Figure 5.5: Usability Evaluation Framework Adopted for Co-PPGIS Prototype ..................................................141

Figure 5.6: Measurement Tools Used in This Study to Determine Usability Indicators ...................................145

Figure 5.7: Graphical Analysis of User Characteristics ....................................................................... 147

Figure 5.8: Graphical Analysis of Usability Indicators Derived from User Performance ..................................148

Figure 5.9: Graphical Analysis of Correlation Results of User Characteristics with Usability Indicators ...............150

Figure 5.10: Correlation (Graph) of IT Knowledge with Usability Indicators 


\section{List of Abbreviations and Acronyms}

\begin{tabular}{|c|c|}
\hline AJAX & Asynchronous JavaScript and XML \\
\hline ANOVA & Analysis of Variance \\
\hline API & Application Programming Interface \\
\hline Co-PPGIS & Collaborative PPGIS \\
\hline CAPE & Co-PPGIS Asynchronous Participatory Environment \\
\hline CASE & Computer Aided Software Engineering \\
\hline CIAC & Citizen Involvement Advisory Committee \\
\hline CMS & Content Management System \\
\hline CSPE & Co-PPGIS Synchronous Participatory Environment \\
\hline CSS & Cascading Style Sheets \\
\hline $\mathrm{CSCW}$ & Computer Supported Cooperative Work \\
\hline DFM & Data Flow Model \\
\hline DHTML & Dynamic Hypertext Markup Language \\
\hline DIV & Division Element \\
\hline ESRI & Environment Source Research Institute \\
\hline GeoDF & GIS-enabled Online Discussion Forum \\
\hline GeoVPMS & GIS-enabled Virtual Public Meeting Space \\
\hline GI & Geographic Information \\
\hline GIS & Geographic Information System \\
\hline GIT & Geographic Information Technology \\
\hline GPA & General Public License \\
\hline GTA & Greater Toronto Area \\
\hline GUI & Graphical User Interface \\
\hline HCI & Human-Computer Interaction \\
\hline HTML & Hypertext Markup Language \\
\hline HTTP & Hypertext Transfer Protocol \\
\hline ICT & Information and Communications Technology \\
\hline IFM & Informational Flow Model \\
\hline JSP & JavaServer Pages \\
\hline LAN & Local Area Network \\
\hline OGC & Open Geospatial Consortium \\
\hline OSI & Open Source Initiative \\
\hline OSS & Open Source Software \\
\hline OOSE & Object Oriented Software Engineering \\
\hline PDF & Portable Document Format \\
\hline
\end{tabular}


PHP

PHPBB

PGIS

PPGIS

PSS

QA

RDBMS

RTMP

SDLC

SDSS

SDK

SPSS

SQL

UML

UMN

URL

WFS

WMS

WWW

XML
PHP Hypertext Pre-processor

PHP Built-in Board

Participatory GIS

Public Participation Geographic Information Systems

Planning Support System

Quality Assurance

Rational Database Management System

Real-time Messaging Protocol

Software Development Life Cycle

Spatial Decision Support System

Software Development Kit

Statistical Package for the Social Sciences

Structured Query language

Unified Modeling Language

University of Minnesota

Uniform Resource Locator

Web Feature Service

Web Map Service

World Wide Web

Extensible Markup Language 


\section{Chapter 1 Introduction}

Municipal planning and development is an important and complex decision-making practice for every local/municipal government. Nowadays, major concerns of researchers, practitioners, and/or government officials are finding ways to increase public participation during planning and development-related workflows. Such issues may be resolved by enhancing Web-based public participation during planning practices. According to Huxhold (1991), good decisions are the best composition of good knowledge and information. Based on a survey by Lowndes et al. (2001), public meetings still are one of the major means of improving public participation in municipal planning related activities. It is true to some extent that public participation relies on efficient notification, participation and frequent feedback sessions. In the era of a modern technology, holding public meetings or establishing Geographic Information System (GIS), Web Technology and World Wide Web (WWW)-based public information centres virtually would be considered as the enhanced ways of gaining public input for the planning and development of a municipality (Tang 2006).

As stated by Meredith (2000), appropriate ways of gathering data and information, robust connection to decisionmaking process, and better tools for getting input into the decision-making process can play a vital role in public participation. It has been perceived that the recent advancement in the communication technologies of the global village, WWW, GIT, and GIS have changed many facets of the traditional way of public participation (Allen 2003). According to Sieber (2006), PPGIS can be defined as a system that brings the academic practices of GIS and mapping to the local level in order to promote knowledge and information. She refers to two perspectives that are currently debated in PPGIS use. The first is top-down (as top-down PPGIS), in which the public are involved less in active participation and it is used only as means of mapping individuals based on socio-economic and demographic criteria with the purpose of analyzing the spatial differences in access to social services. It plays an important role by making adjustments for the deficiencies and improvements in public management. The second perspective is bottom-up that is referred to as a counter method of PPGIS. Its purpose is to work with the public in order to let them learn the technologies; and in turn produce their own GIS.

PPGIS provides a Web interface for spatial/attribute analyses with public input, which leads to better decisionmaking (Wong and Chua, 2001). It enables all the citizens to participate freely in municipal development projects by exploring geospatial data over the WWW (Evans et al. 1999; Hackney's GIS Team 2004; Orlando GIS 2004). Moreover, a growing number of Web-based public participation GIS tools and Planning Support Systems (PSS) have been recently developed to support public participation and decision-making practices in various planning processes. Many of these tools have been used with the integration or collaboration of other decision-making tools 
in order to achieve the maximum benefits. In most cases, such systems are based on existing commercial and proprietary software tools, while some are still in the research prototype stage. More recently, open source solutions have rapidly been adopted in the development of Participatory GIS (PGIS) applications. PSS is also designed for planners according to their requirements, but primarily focuses on coupling computer analytical tools and simulation models with visual displays for land-use, transportation development options, and environmental consequences of the available choices and their alternatives ways (Brail et al. 2001).

To promote this kind of collaborative system, multi-user collaboration has increasingly been integrated into many tasks by involving people from different organizations in which maps often play a central role for providing visual information to support collaborative decision-making over the web. It can be achieved with the help of the rapidly expanding range of Web-based technologies. In addition, a synchronous approach has been developed to support collaboration among stakeholders/users (Chang 2010; Chung et al. 1994; Begole et al. 1999); however, little research has been done on designing and developing synchronous collaborative applications such as Open Source Software (OSS)-based online map-sharing tools in order to support real-time collaboration. By examining the researchers' contributions from the literature review, this study seeks to outline the significance of the implementation of valuable and adequate methods/procedures, tools, and techniques to fill in the existing gaps (i.e., issues in current public participation practices). Multi-user synchronous communications or discussions among the stakeholders often improve the understanding regarding any municipal project by frequent feedback sessions for effective and better decision-making (Evans et al. 1999; Ventura et al. 2002; Tang 2006; Bryant and Wilcox 2006; MacEachren et al. 2001; Li et al. 2007; Jankowski and Nyerges 2001 a; Jankowski and Nyerges 2003).

This research aims to develop a real-time Co-PPGIS map-sharing infrastructure, which includes both synchronous and asynchronous approaches to public participation, with the option of integrating local data for enhancing input of multiple stakeholders during discussion or feedback sessions. It focuses on not only real-time map (or geospatial information) sharing, but also integration of other open source-based groupware solutions, which are helpful in promoting public participation culture in municipality-related workflows and others sectors. The objective of this study is to make sure that the Co-PPGIS will help stakeholders to increase their participation in any project and/or support decision makers in reaching a final and better decision efficiently. In this context, the enhanced Co-PPGIS integrates social collaboration tools, Web-mapping functionalities, and Human-Computer Interaction (HCI) principles. In brief, Co-PPGIS is the integration of various types of open source based modules and open map services (i.e., Google, Yahoo, and Bing, etc.) to provide collaborative real-time map-sharing infrastructure to support and/or enhance public input during planning and decision making-related activities. 
An examination of existing practices of getting the public to participate in municipal development projects reveals that insufficient prior access to information (before public meeting starts) and lack of effective, innovative communication channels other than attending public meetings still remain the major hurdles in the enhancement of public input ( $\mathrm{Li}$ et al. 2004). The real-time collaborative GIS, GIS-supported groupware, Information and Communications Technology (ICT), and Internet-based CSCW concepts are increasingly used during participationrelated tasks allowing synchronous behaviour among concerned groups of stakeholders anywhere and anytime. Moreover, Web-based PPGIS tools have now been recognized as an efficient component of sound planning processes among others. However, any PSS cannot exist alone without GIS (Klosterman 2001). Therefore, PPGIS must integrate with other existing information technologies for the better solution of existing challenges related to municipal planning. In addition, as part of the research, the prototype's usability has been evaluated by providing a case study involving concerned participants with planning issues. The main purpose of this usability evaluation is to find out the productive results, which will conclude that Co-PPGIS to provide cost effective solutions and significant support in enhancing public participation, as well as improving a better decision-making process after the integration and development with Internet-based GIS and OSS technologies.

\subsection{Research Context}

\subsubsection{Central Idea}

This research represents an effort to develop an integrated Co-PPGIS map-sharing infrastructure (to improve existing PPGIS application) that facilitates online public participation in municipal planning and management processes. Special attention is paid to the integration of different Web-based information and groupware technologies with GIS functions, to provide tools for: 1) efficient participation mediation and frequent feedback, 2) automated participation and decision-making procedures; and 3) synchronous and asynchronous collaboration among stakeholders for better understanding, communication, and effective decision-making. In addition, a research prototype has been developed, based on collaborative approaches such as a synchronous participatory approach, for the implementation of Web GIS-based collaborative map-sharing mechanism. This synchronous approach provides a real-time map-sharing mechanism, which was developed by adopting similar functionalities provided by CSCW and/or groupware tools. On the other hand, the asynchronous approach was developed using Web GIS collaborative forums-based techniques to support public input or discussion. The objective of both approaches is to gain public participation in all phases of municipal planning context. Although the Co-PPGIS supports both GIS-based asynchronous and synchronous participation approaches, more emphasis was placed on synchronous participation, which provides innovative ways of involving the public in the planning process using a real-time virtual public meeting platform (Wong and Chua 2001; Li et al. 2007; Butt and Li 2012). 


\subsubsection{Issues and Concerns in Existing Participation Practices and PPGIS}

This research addresses the problems, to a certain extent, within existing PPGIS and public participation practices during municipal planning processes and management tasks. During the existing practice of the municipal planning, public participation is slightly limited, as it depends upon number of notifications sent and number of participants in attendance at the meeting. The reason for this deficiency is that the communication channels are less effective and interactive with the existing practice, especially in the case of exploring spatial data among a group of individuals. During the public meeting, planning workflows and models are presented to participants, which are made of paper and pasteboard, hard copy maps, perspective drawings, and combinations of two or more photographs, in order to publicize a design planning solution (Kingston 2002; Li et al. 2004). Despite the growth in Information Technology (IT) and development of various PPGIS frameworks, there are still some issues and concerns related to current public participation workflows of municipal planning as well as participatory GIS-based applications developed, which need to be rectified. Figure 1.1 summarizes the issues and concerns identified by evaluating the current practices of municipal planning, and the PPGIS found in the literature. 


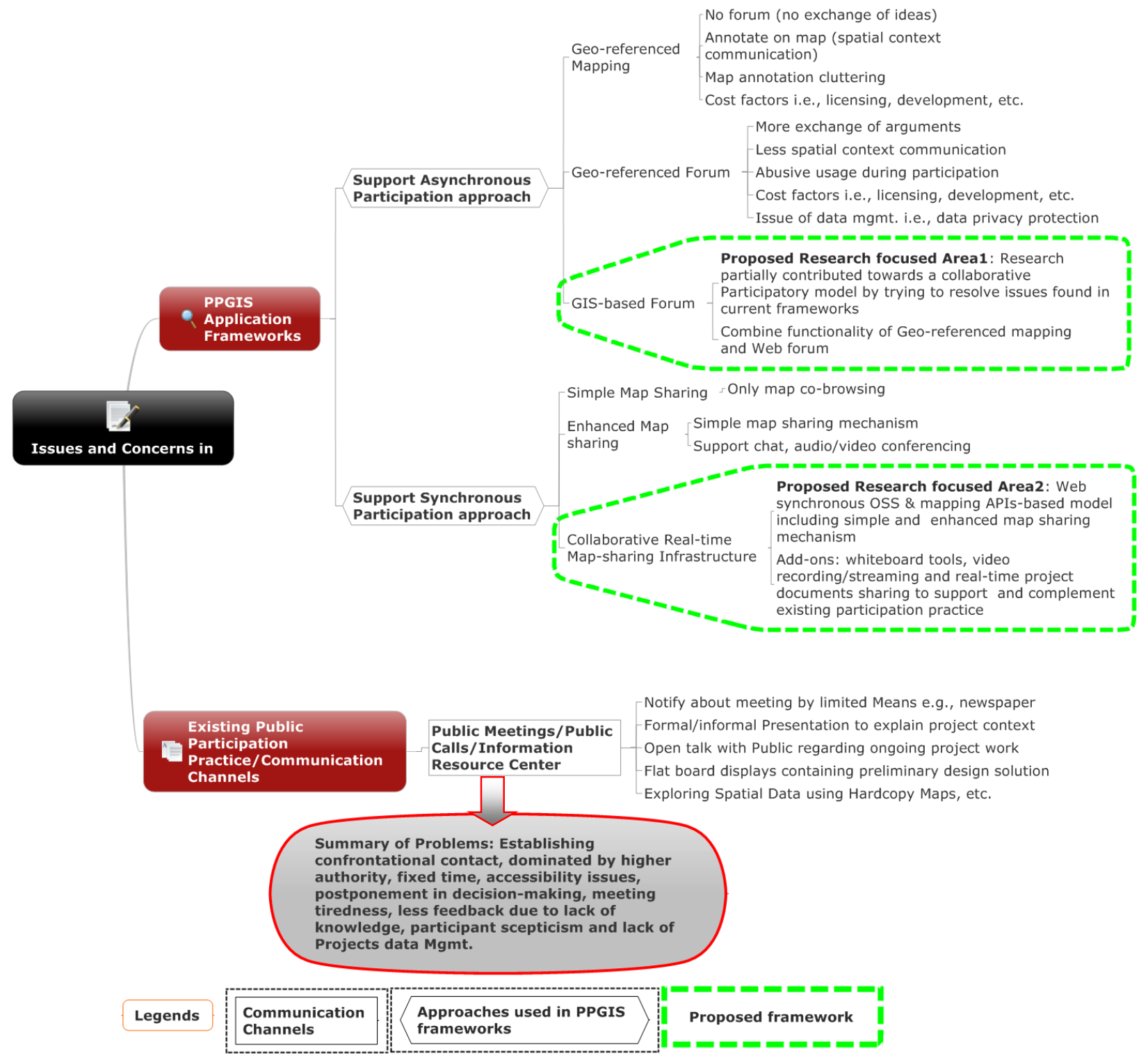

Figure 1.1: Issues and Concerns in Existing Practices of Public Participation

The bottom portion of Figure 1.1 shows the communication channels and summary of their related problems in relation to current public participation practice. For example, public meetings may come up with limited notification channels and awareness mechanisms, insufficient access to the information required for public input, establishing confrontational contact, higher authority saturation, meeting tiredness, fixed time and accessibility issues, lack of a more effective, innovative platform for communications and exchange of ideas and information, which all-together 
result in delays in decision-making. The upper region discusses issues connected to major participatory approaches supported by current/existing PPGIS applications. It categorizes the PPGIS application frameworks that support synchronous and asynchronous participatory approaches. In the past, PPGIS applications supported only the asynchronous participatory approach, which introduced and supported GIS referenced mapping and forums-based techniques. The following discussions help identify issues and concerns in the existing PPGIS application frameworks that support asynchronous public participation.

Communication Channels: There is a lack of efficient communication channels for the enhancement of public participation during existing planning and development practices. For instance, exploring or accessing municipal project information resources in a spatial context during public meetings to discuss participant's point of views is still one of the concerns. Recent web-based PPGIS only supports participation needs to some extent. For example, public participants can view, edit, print, and download spatial or non-spatial data related to the ongoing and future municipal projects using a geo-referenced map. In addition, the public can share their input during the forum-based discussion anytime, anywhere. Both approaches are useful to facilitate discussion among participants, sharing of data such as maps and information related to municipal projects. According to Evans et al. (1999) and Ventura et al. (2002), only a few PPGIS allow the public or stakeholders to post their annotations and exchange their ideas efficiently, but they usually do not support participation on a real-time basis during ongoing public meetings. For instance, the Argumentation Map included participation forums to support multi-way communication among the participants and decision makers (Rinner 1999; Keßler 2004; Keßler et al. 2005; $\mathrm{Li}$ and $\mathrm{Ma}$ 2006). However, providing access to the municipal project's information before, during, and after the meeting is not enough for the public to be fully involved or engaged in the complete planning process. Thus, there is a need for a synchronous collaborative map-sharing meeting mechanism in order to enhance and support real-time public participation during municipal planning workflows.

Cost: The implementation of Web-based PPGIS applications in small municipalities, especially using commercial technologies, is somewhat costly for local governments. Therefore, insufficient budget often becomes one of the major hindrances in the design, development, and implementation of GIS-based public participation applications in such municipalities. Therefore, the needs for empirical implementation experience using a framework that can potentially reduce the cost of PPGIS design and implementation is required.

Abusive Usage and Data Privacy Protection: Developers of PPGIS applications have carried out some protective measures, such as setting rights and privileges of participants, which have the ability to impede any abusive usage, and use of offensive language and unpleasant multimedia contents during online forum-based participation. However, sharing and privacy protection of municipal project data is still one of the concerns, especially when sharing spatial/attribute data of municipal projects among public participants. 
Lack of Support for Quick Decision-Making: Another purpose of designing and implementing PPGIS (besides increasing public participation) is to collect the public input, comments, and correspondences in a timely manner, which may be helpful in reducing the span of a new municipal planning project. Public participation does not automatically lead to better decisions, however. Decision-making authorities may use public feedback for making effective decisions and/or improve decision-making by ensuring that their decisions have been based on shared knowledge and experiences (Blaschke 2004). Although this issue has been addressed, to some extent, it requires further investigation by researchers together with actively involved end users or authorities.

Projects Information Management: Current PPGIS applications lack ability of managing the project information (i.e., notification, project progress, tracking status, informative documents, reports that may be used for future references and development activities) related to municipal planning. This leads to time-consuming or unreliable approaches of transferring data and other municipality project-related documents among project participants.

The proposed areas of interest in the design and development of a research model are shown with dashed lines (highlighted as green in colour) in Figure 1.1. In addition, the proposed areas depict the research contribution and/or enhancement of the existing PPGIS frameworks by providing the enhanced GIS-based forum and virtual meeting infrastructures. Furthermore, the above issues must address the effective output or success of developing or implementing an application framework, in which the public and municipal bodies can participate and share their ideas in both synchronous and asynchronous fashions during municipal planning and decision-making contexts.

\subsubsection{Web-based Real-time Co-PPGIS as a Potential Solution}

The real-time Co-PPGIS considers public participation as a continuous input that feeds valuable information into all processes related to municipal planning and management. It provides the potential integration of PSS, PPGIS, realtime $\mathrm{CSCW}$, and groupware into existing public participation practices to empower local communities, increase public participation, and broaden the use of geospatial information by the public for better decision-making.

The major functions of the real-time Co-PPGIS include:

1. Collaborative browsing, editing and manipulating of spatial as well as textual data related to municipal planning and management (in both asynchronous and synchronous participatory approaches);

2. Collaborative sharing or dissemination of municipal projects information;

3. Real-time communications (map/spatial feature-based chatting, rich multimedia content sharing, audio/video conferencing, online virtual public meeting space, mobile/tablet device-based instant, anytime, anywhere data sharing and/or android device-based geo-tagging); and 
4. Enhanced municipal planning process workflows for better-coordinated public participation, collaborative and effective decision-making.

Similarly, the implementation of such a collaborative PPGIS infrastructure may be comparatively easy to realize in order to deliver the required functional/non-functional support. As a proof of concepts, Co-PPGIS is trying to develop a cost effective, interactive, and user-friendly application with the implementation of the following attributes:

- Requirements, Analysis and Design using Unified Modeling Language (UML ${ }^{1}$ ): The UML-based requirements can be captured by having dialogues with municipal bodies about their needs, understanding, and visualizing the existing workflows of municipal planning in relation to public participation and decision-making as well as by evaluating existing PPGIS application frameworks.

- Virtual Resource of Information: The Co-PPGIS provides a platform of electronic resources of documentations of municipal regions (i.e., documents, maps and pictures) related to the under-construction and future projects, minutes of the meetings, by-laws or any other detailed information/documents.

- Communication Channels (asynchronous and synchronous communication): The Co-PPGIS can be bundled with a variety of communicative and participatory channels: exploration of spatial data, GIS-based forum communication, feedback sessions, notification, focus group discussion/collaborating Web meeting, videobased chatting and conferencing with meeting presenters/facilitators, and mapping Application Programming Interface (APIs). All these approaches provide easy accessibility to meeting places and data related to available and future municipality projects for public participation in order to understand the better state of their thoughts and opinions using a single GIS-enabled platform.

- User-friendly Interface: The Co-PPGIS prototype is designed by following sets of rules defined by HumanComputer Interaction (HCI) to make it fully useable and user-friendly for the public to get a maximum benefit. Moreover, to come up with the successful user-friendly and interactive interfaces, the prototype designing stage can be developed by the following HCI rules:

1. Strive for Consistency: Using Cascading Style Sheets (CSS) consistent color, layout, background, capitalization, and fonts will help ensure interface consistency.

2. Offer Informative Feedback: Using Dynamic Hypertext Markup Language (DHTML)-based visual effects (e.g., dynamically color changing of buttons, backgrounds and presenting extra information with tool tips) the system will be able to respond in an effective way to every user's action.

${ }^{1}$ The UML is a unified modeling language which is designed to provide a standard way to visualize the design of a system and helps to understand the system requirements in the field of software engineering to comprehend software development lifecycle with its building blocks i.e., requirement, analysis, and design. 
3. Offer Error Prevention and Simple Error Handling: Server and client side scripts written in PHP Hypertext Pre-processor (PHP) and JavaScript languages will be used to make this feature available. For instance, JavaScript-based attractive popup windows will be used for error handling and prevention during the user interaction (i.e., wrong password input, etc.) with a spatial database.

- Usability in Business Process Management - Content Management System (CMS): Due to the design and implementation of CMS-based modular component, the research prototype provides the convenience to the facilitator for managing Web contents and business processes online.

- Platform Independent Environment: The Co-PPGIS prototype is designed to run on any kind of operating system using compatible web browsers.

- User's Perspective - Good Response Time: It is one of the core requirements of the end-users before deployment of the system. An object-oriented, component-based modeling technique is used to boost up the required response time.

- System's Perspective - Maximum Throughput: The distributed database concept is used to get a maximum throughput from the Spatial Database Management System (SDBMS). The major reason of using the objectrelational SDBMS (e.g., PostGIS) is to get the maximum (quick) rate of transferring spatial data while developing any GIS-based application framework.

- Relatively Cost-Effective Solution: A prototype is designed and implemented using a unified modeling approach to demonstrate a GIS-based architecture with utilization of various open source GIS and other OSS tools to make it cost-effective for smaller budget municipalities.

Successful implementation of each above key attribute as an input function during the prototype development life cycle provides a favourable and desired design output in the end.

\subsection{Research Objectives}

Over the past few years, most of the PPGIS models have been focusing on supporting asynchronous participation approaches, e.g., public participation using geo-referenced forums that support geo-referenced mapping (Rinner 2001; Balram 2004; Li et al. 2007). Efforts have also been taken in seeking and developing improved and effective infrastructure of public participatory GIS efficiently. The primary research goal of this study is to develop a Webbased collaborative PPGIS infrastructure in order to support municipal planning through a better decision-making process. In order to achieve this goal, this research focuses on accomplishing the following objectives: 
1. To design a real-time Co-PPGIS that can employ open source geospatial data, standards, software tools and Web services.

2. To develop and implement a prototype based on the collaborative, Web GIS-based architecture, which includes a GIS-based forum, a notification/feedback component, mapping APIs-based spatial component, Web blogs and collaborative Web map-sharing infrastructure that helps the stakeholders to share their multiple ideas in real-time scenarios without leaving their work location.

3. To evaluate the usability of the Co-PPGIS prototype using a case study scenario: a mock-up scenario testing approach related to municipal planning to identify design evaluation, to define possible improvements, and to model potential impact of the synchronous/asynchronous collaborative mechanism for public participation in municipal planning processes.

\subsection{Overview of Research Methodology}

This section presents an overview of the research methodology. Comprehensive study of user's requirements or understanding of the related literature from both researchers and end user's perspective is essential to ensure a successful design, development and implementation of an effective system. Figure 1.2 depicts the synopsis of the research methodology used in this thesis that is comprised of five building blocks.

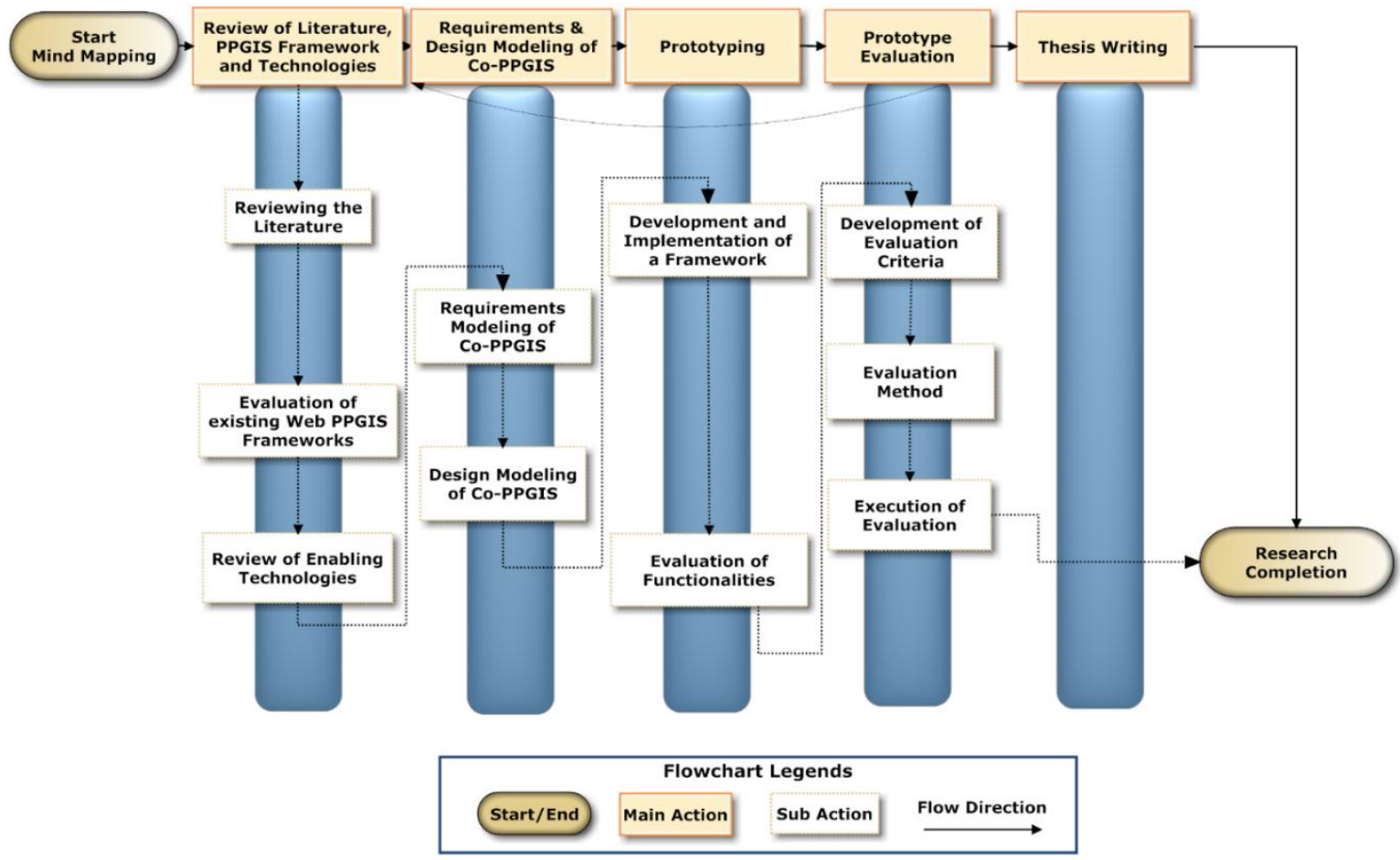

Figure 1.2: An Overview of the Research Methodology 
In this research, the major steps have been implemented along with its sub-activities to achieve the research objectives and goals. The steps performed to accomplish research objectives are described briefly as follows:

\subsubsection{Review of Literature, PPGIS Frameworks and Technologies}

Reviewing the Literature: The research started by focusing on a detailed literature review to build necessary knowledge for the proposed areas of study. Mainly, this step of the research tries to build preliminary knowledge, which is ultimately helpful in achieving the objectives of the research. It is organized by gathering relevant literature for the research, especially to obtain a general understanding of the existing planning practices and the collection of technical specifications related to the frameworks of Web-based PPGIS. With the help of a better understanding of PPGIS and municipal planning practices, their constraints and requirements can be modeled by involving the public.

Evaluation of Existing Web-PPGIS Frameworks: The existing PPGIS application frameworks are evaluated and compared by following the statement "more detailed analysis ends up with effective outcomes" (Bennett et al. 2005). The main idea of this phase is to identify the problems/gaps in existing PPGIS application frameworks. In addition, this will help in assessing and identifying the factors that will be helpful for supporting further analysis and modeling of the proposed solutions. The main tasks performed in this step are: (1) acquire more in-depth understanding of the nature of existing participation practices and municipal planning and development related workflows; (2) build understanding and knowledge about limitations and requirements of existing PPGIS; (3) review and identify existing online PPGIS system's functionalities; and (4) identify the collaboration requirements of participation planning.

Review of Enabling Technologies: Before making a selection of technologies to be used in the development of the system, it is better to understand first what is needed, what is attainable and what would be suitable or the best fit for the required/preferable design model. Moreover, the comparative analysis of the pros and cons of the enabling technologies is performed in this step.

\subsubsection{Requirements and Design Modeling of Co-PPGIS}

This step of the research methodology is mainly focused on achieving the first objective.

Requirement Modeling of Co-PPGIS: Another important step is to design requirement model (requirements specification) using Object Oriented Software Engineering (OOSE). It is stated that collaborative GIS designs share many common ideas with object-oriented systems. The use of OOSE is innovative and helpful for modeling a collaborative PPGIS design ( $\mathrm{Li}$ et al. 2004; Booch 2005; Ma (2006); Balram and Dragicevic 2006a, 2006b). The 
requirements are gathered by evaluating the existing Web-PPGIS applications and by analyzing the related planning processes. In order to do so, the following actions need to be taken for the design of a successful requirements model of a research framework:

- Obtain insights of existing public participation practices by gaining understanding through a literature review connected to research context, and if necessary by having dialogues with municipal bodies as well as attending public meetings.

- Compare functionalities, participatory approaches and research models supported by various online PPGIS applications in relation to these requirements (see Sections 2.3.2 and 2.3.3).

Design Modeling of Co-PPGIS: This part of the research captures all the necessary building blocks for the analysis and designing of a GIS-based public collaborative participatory infrastructure. Based on the outcomes, the real-time Co-PPGIS prototype components are designed using UML.

\subsubsection{Prototyping}

This step of the research methodology is focused on achieving the second objective.

Development and Implementation of Co-PPGIS: A Co-PPGIS prototype is developed and implemented as a proof of concept at this stage.

Prototype Initial-level Functionalities Check: At this step, functionalities of user-friendly interfaces are evaluated and checked to realize the integration between different components of the Co-PPGIS. If necessary, the system is showcased to the end-users/stakeholders to gather preliminary feedback/evaluation.

\subsubsection{Prototype Evaluation}

This step of the research methodology is to address the third objective. The purpose of this step is to evaluate the usability of the Co-PPGIS prototype components with the help of a mockup case study scenario. In order to carry out the evaluation, a set of evaluation criteria is established and the evaluated method needs to be developed.

\subsection{Organization of the Thesis}

This thesis is organized into six chapters. Chapter 1 provides an overview of the present PPGIS research with its significance. It also describes the goals, objectives and an overview of research methodology adopted during this research. 
Chapter 2 discusses the related theories and principles of public participation within the contexts of municipal planning and decision-making. It provides the background knowledge to the present research followed by the discussion related to the usage and benefits of the open-source software technologies.

Chapter 3 discusses the requirements and analysis modeling of Co-PPGIS using object engineering software engineering approaches including the intended use, target user group, and user requirements. It also presents the design requirements for modeling Co-PPGIS (e.g., a concept and understanding of Co-PPIGS along with its architectural design).

Chapter 4 depicts the design methodology of Co-PPGIS service-oriented architecture. The details about the implementation and demonstration of the prototype are discussed. It also covers the demonstration of the prototype Co-PPGIS to support real-time map-sharing mechanism using mock-up case scenarios.

Chapter 5 describes the usability evaluation of prototype using a mock case study. Chapter 6 concludes and summarizes work completed in this research, followed by the research contribution and significance and then suggestions or recommendations are discussed by summarizing the opportunities for future research. 


\section{Chapter 2 Background and Literature Review}

This chapter mainly focuses on the importance of public participation in decision-making processes during municipal planning and developments. Public or local stakeholders can be involved more effectively through the integration of GIS technologies into these processes. Traditional methods of public participation (i.e., public meetings) are integrated into some PPGIS projects to collect public ideas, values and preferences (Hopkins et al., 2004). Collaborative use of GIS-based services not only involves the public and planners in the decision-making process, but also assists in exchanging ideas and building local spatial knowledge. It provides an opportunity to local stakeholder to get instant access and visualize the spatial information while participating in the decision-making process (Healey 1997). Butt and Li (2014) states that increased public participation can lead to better and effective decision-making. In addition, decision-making and public participation processes have a direct relationship. For example, better decision-making processes can also lead to an increase in a user's participation.

\subsection{Concepts of Public Participation in Planning and Management}

This section briefly describes: 1) the concepts of public participation, 2) degree of public involvement, and 3) existing public participation practice during municipal planning related activities.

\subsubsection{Concepts of Public Participation}

The concept of public participation can be realized through an interactive and iterative process of public meetings and reviews, of which the public or the interested agencies are notified via public notifications. This kind of process provides opportunities for public participation, through public meetings/focus group discussions, interviews, telephone inquiries, letters, emails and faxes. Essentially, the objective of any participatory process is to identify the needs and availability of the people, and bring them forth in a consensus. Moreover, participatory processes often come with an integrated platform to promote a two-way learning process among the local citizens, political commitment/corporations and local authorities regarding any municipal project. For instance, public participation in e-government provides one of the primary participation avenues, which is designed to make it easier for citizens, business interests, municipalities, other authorities (private agencies), non-governmental organizations and government agencies to access government information and services to create new policies for better decisionmaking. 
Figure 2.1, developed based on McClure (2000), shows different components of e-government framework. In addition, it conceptually describes the e-government framework, in which all stakeholders are interacting with each other electronically to support the planning and development process in an effective and efficient way.

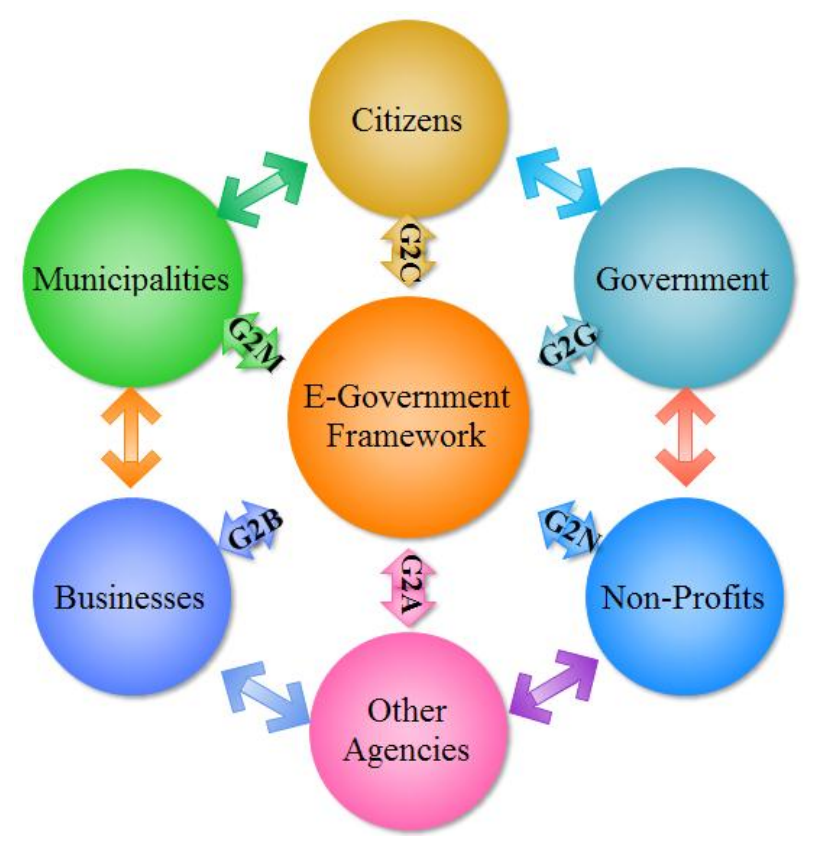

Figure 2.1: Modified Conceptual Diagram of E-government Framework (McClure 2000)

\subsubsection{Degrees of Public Participation}

Degree of public participation refers to the extent to which the public or local stakeholders are involved in a decision-making process. It can be described with the help of a ladder of participation.

Public participation ladders are widely used by many researchers to describe and create a better understanding of the degree of public participation. Firstly introduced by Arnstein (1969), a ladder structure is used to depict the different levels of power or rights given to citizens. In addition, the ladder explains how power is re-distributed between citizens and power holders using a public participation strategy within the planning and development process. This ladder leads to a better outcome of the planning process concerning the public participatory approach (Arnstein 1969).

The bottom rungs of Arnstein's ladder (manipulation and therapy) represent the non-participative status of citizens since they cannot participate in planning and development processes at this level. The top rungs of the ladder (delegate power and citizen control stage) represent full citizen control with managerial power. These citizens 
handle the responsibilities of final planning, policymaking and decision-making related tasks (Arnstein 1969; Carver 2001).

Figure 2.2, developed based on Arnstein (1969), shows eight rungs on the ladder of public participation. Moreover, for the ease of orientation and better understanding, the characteristics of important rungs are shown on Arnstein's ladder of participation.

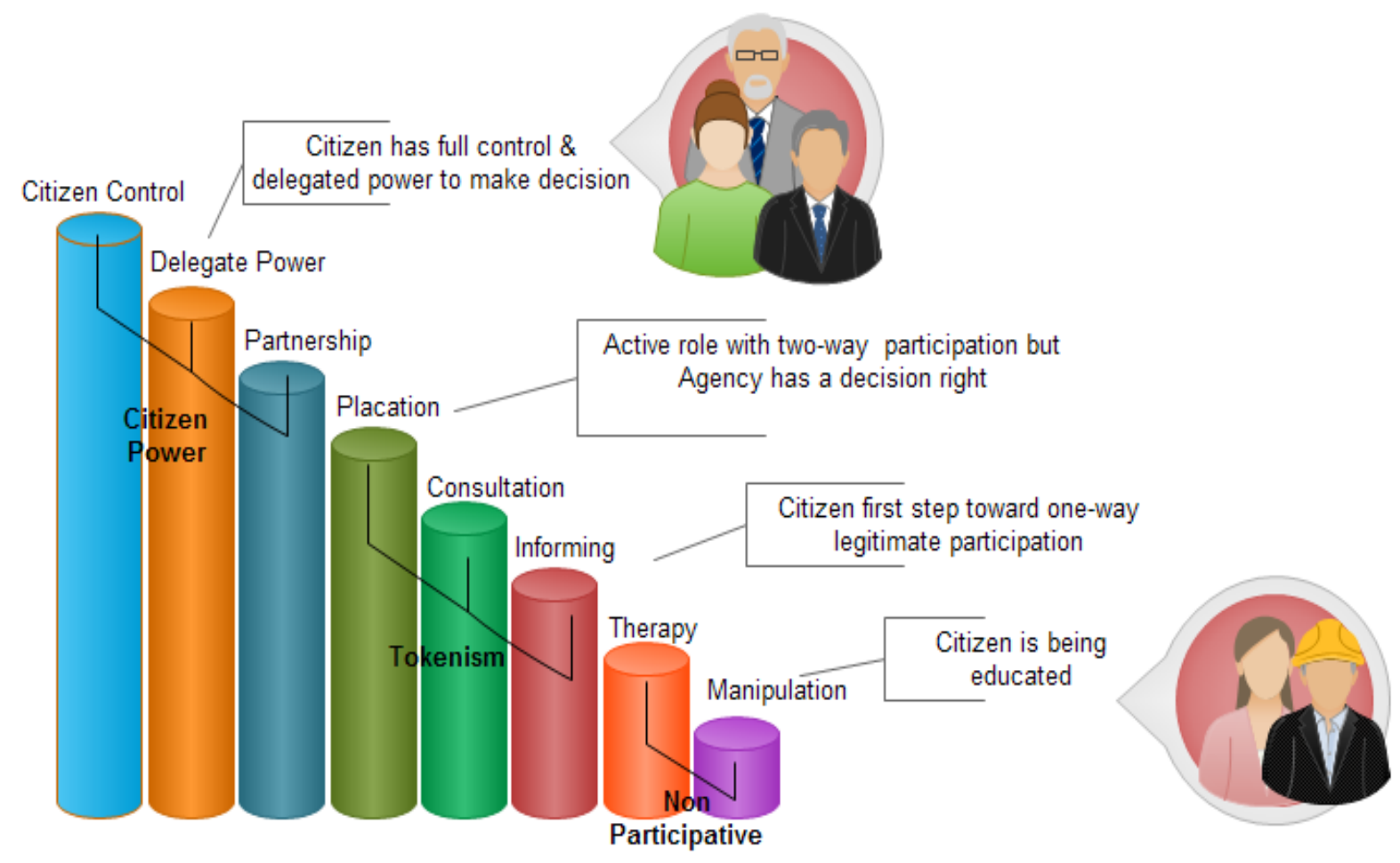

Ladder of Participation

Figure 2.2: Modified Ladder of Public Participation (Arnstein 1969)

Based on Arnstein's ladder, Weidemann and Fermers (1993) reproduced a six-rung ladder of public participation, which deals with the people' rights/powers and the decision-making authorities. 
Figure 2.3, developed based on Weidemann and Fermers (1993), shows different rungs and important characteristics of Weidemann and Fermers's ladder of public participation.

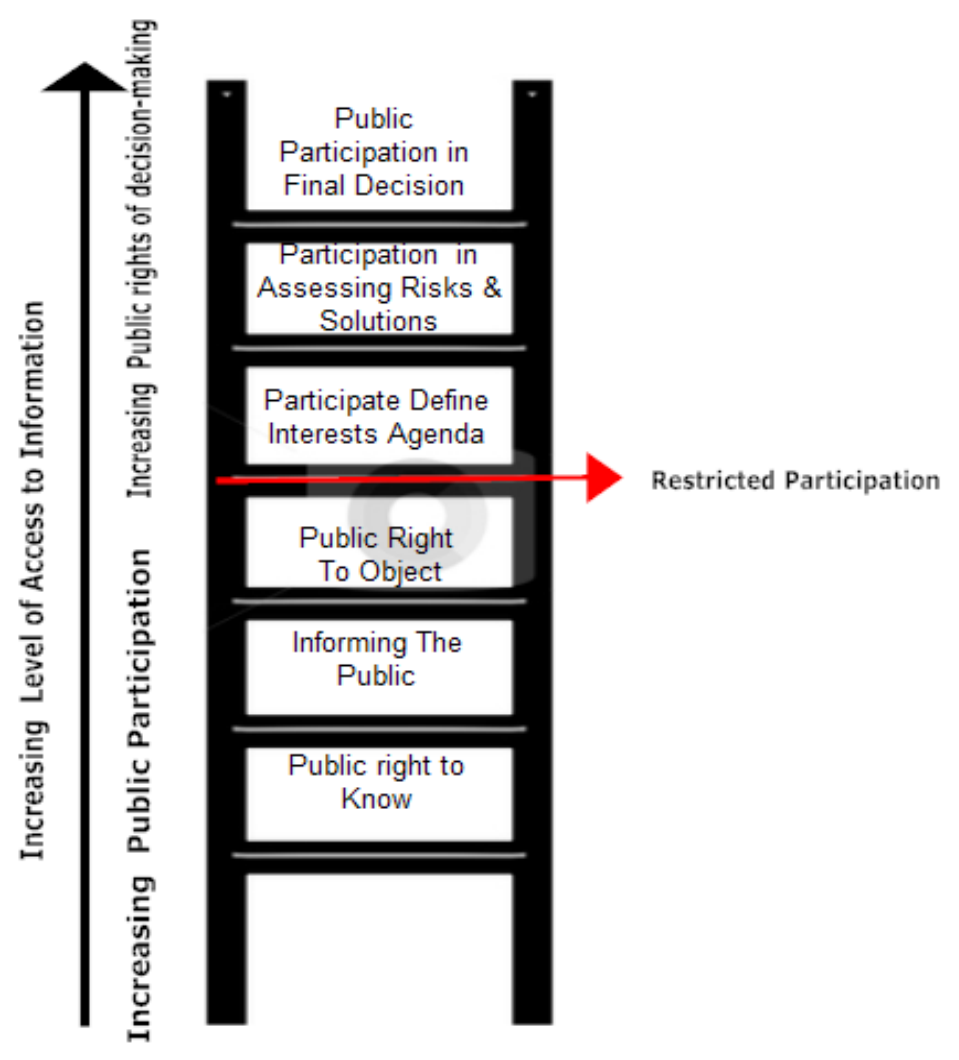

Figure 2.3: Modified Public Participation Ladder (Wiedemann and Fermers 1993)

Arnstein's ladder provides significant public participation by involving citizens at different levels with respect to their knowledge and expertise (Butt and Li 2014). However, it was criticized because it only focuses on the power relations within the consultation/ participation process and fails to highlight the significant consultation aspects such as "how much understanding of people's needs and values is enough"; "how much people can really be involved in decisions that are often highly technical"; and finally, "how much participation is needed to be effective?" (Shipley and Utz 2012; Tritter and McCallum 2006). Wiedermann and Femer's ladder of participation focuses on administrative participation with collaboration of governmental agencies within a spectrum from education to joint decision-making to create new policies (Wiedemann and Femers 1993). Simply stated, knowing these degrees of the public participation (i.e., increases the level of access to information) make it possible to cut through the 
exaggeration in order to better understand the increasingly strident demands for participation, and the gamut of confusing responses from the decision authorities (Wiedemann and Fermers 1993). Therefore, public participation should be considered a continuous input process that feeds significant information into all processes related to planning and development.

Furthermore, the Oregon State Citizen Involvement Advisory Committee (CIAC) provides reasons (Lynn and Busenberg 1995; CIAC 2008) that describe the significance of public participation during planning and development processes followed by discussions of recognized theories of public participation ladders developed by Arnstein (1969) and Wiedemann and Femers (1993). The Oregon State CIAC provides five major reasons why citizens should have the opportunity to participate in planning and development and how these processes increase its versatility along with the public participation as a key input source (Lynn and Busenberg 1995; Vari 1996; Sullivan 1998; Sadagopan 2000). Figure 2.4, developed based on CIAC (2008), illustrates the reasons of the CIAC in a brief and self-explanatory way.

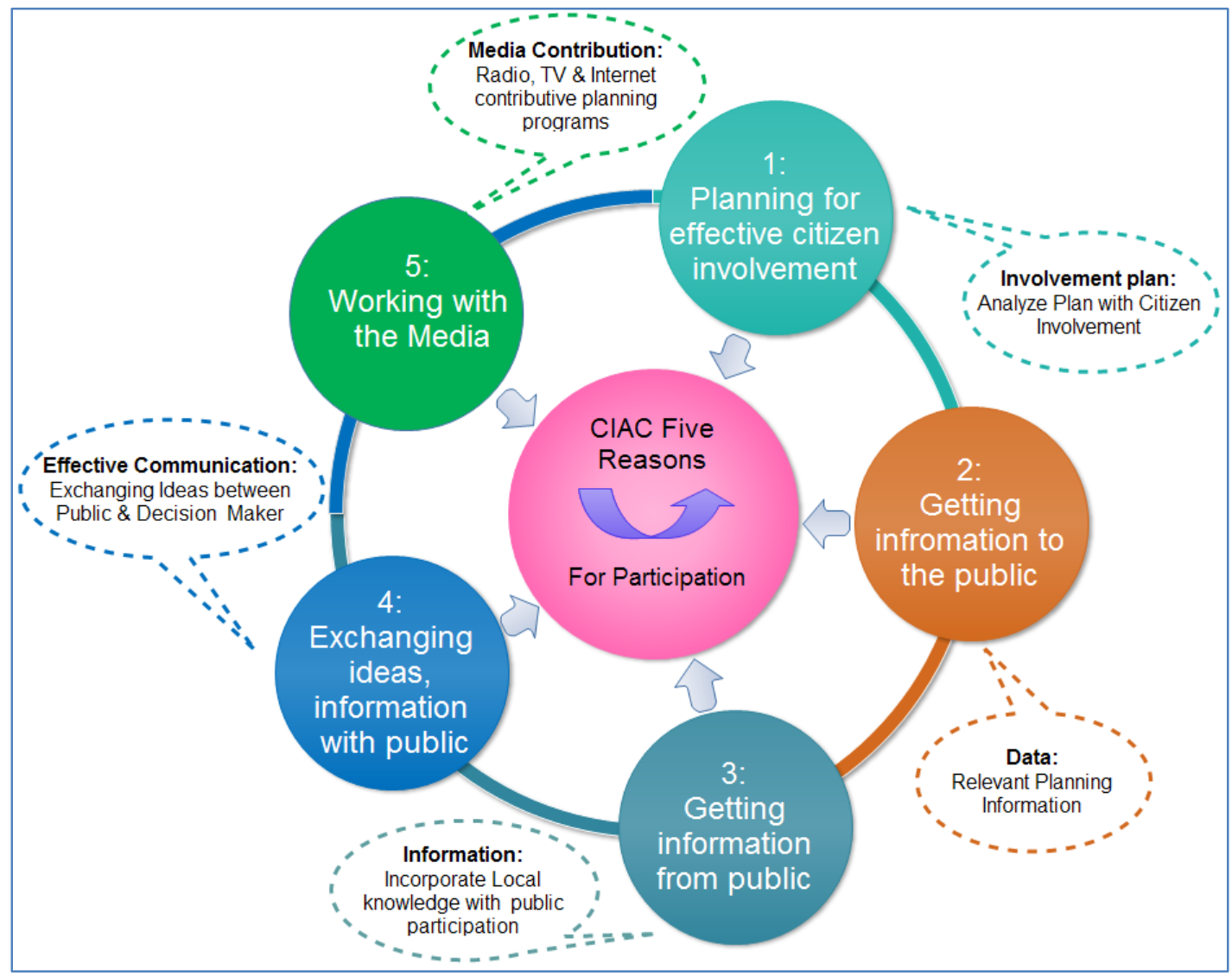

Figure 2.4: Modified Five Reasons of Participation by CIAC (http://www.oregon.gov) 
The CIAC (Figure 2.4) states that the strategy for citizen participation, getting information to and from the stakeholders, and exchanging ideas with e-media contribution effectively and efficiently facilitate the path that leads to strong public participation.

\subsubsection{Existing Public Participation Practice during Municipal Planning and Development}

The planning process in every field of life has certain goals or objectives. For instance, public participation in developing a large-scale or small-scale municipal plan has some objectives, such as to democratize the planning process, to assist in the conveyance or dissemination of ideas and to support the decision-making process. According to traditional methods, public participation in the municipal planning and management processes ranges from neighbor notifications, interviews, exhibitions, public meetings/focus group discussions, and public enquires through telephone, letters, mails, fax or public hearings (Kingston 2007; Kingston 2002; Li et al. 2004). Therefore, decision makers/planners show their plans on PowerPoint slides/point boards, which is still one of the commonly used participatory approaches, in order to publicize the need of a proposed solution during public meetings (Lowndes et al. 2001).

Holding public meetings to gather public feedback during planning and development-related workflows for better decision-making is one of the common practices in the Western world. For instance, in Europe and North America, it is in the by-laws of many municipalities and local governments to require a certain level of public participation in their decision-making processes (Li et al. 2006). Based on a survey (Lowndes et al. 2001), public participation relies on effective notification, participation, and feedback through public meetings, which are still one of the most identified forms of public participation. 
Table 2.1 shows the issues and concerns in existing practices of public participation during planning and development-related processes.

Table 2.1: Issues and Concerns in Existing Practice of Public Participation

\begin{tabular}{|c|c|c|}
\hline Issues & \multicolumn{2}{|l|}{ Concerns } \\
\hline Notification & \multicolumn{2}{|c|}{ Limited means e.g., newspaper, flyer etc. } \\
\hline \multirow{3}{*}{ *Communication Channels } & \multirow{3}{*}{$\begin{array}{l}\text { Public Meetings / Public } \\
\text { Calls/ Information } \\
\text { Resource Center }\end{array}$} & Formal/informal presentation \\
\hline & & Open talk with public \\
\hline & & $\begin{array}{l}\text { Flat board displays containing preliminary } \\
\text { design/ model solution }\end{array}$ \\
\hline Exploring Spatial Data & \multicolumn{2}{|l|}{ Using hardcopy maps, etc. } \\
\hline \multirow[t]{2}{*}{ Access of Information } & \multicolumn{2}{|c|}{ Less feedback or public participation } \\
\hline & \multicolumn{2}{|c|}{ Lack in projects data management } \\
\hline \multicolumn{3}{|c|}{$\begin{array}{l}\text { *Establishing confrontational contact, dominated by higher authority, fix time, feedback lack anc } \\
\text { accessibility issues }\end{array}$} \\
\hline
\end{tabular}

Table 2.1 depicts an examination of existing public participation practices especially related to notification, communication channels, access of information, and exploring spatial data of municipal projects. The main issues of traditional public participation practices are: (a) limited notification channels and awareness mechanisms, (b) insufficient access to the information required for public input, and (c) lack of a more effective, innovative platform for communications and exchange of ideas and information. According to Baker et al. (2005), the success of public meetings depends on several critical factors, including "effectively notifying and educating the public about the agenda before the hearing, carefully planning and management of the meeting, giving a clear and media-rich presentation of the issues, and conducting an appropriate follow-up".

Public participation can be considered efficient and effective when large numbers of participants understand the message and actively give valuable feedback in the shortest period of time. For efficient public participation, better access to information, better connections to decision-making process and better tools for getting input into a decision-making process are important (Meredith 2000). Effective public participation also requires input from the public to the proponent; output of technical information about the issues from the proponent to the public; and 
exchange of information, perceptions and values between the proponent and the public and among the various public groups in the shortest time period (Connor 1996).

\subsection{Public Participation GIS (PPGIS)}

Public participatory GIS (PPGIS) has been stimulated with the help of Internet and WWW technologies since 1990s (Talen 1999; Kingston et al. 2000; Keßler 2004; Zhao and Coleman 2006). Integration of web and GIS based technologies in a decision-making process to enable e-participation is known as Web-based PPGIS (Web-PPGIS). Electronic participation (e-participation) refers to the voice conferences, e-newsletters, emails, online polling and feedback through forms or conducting sessions, video chatting, online notifications, and discussion forums. PPGIS deals with variety of approaches such as GIS, web and the Internet that enables easily access to relevant spatial and temporal information to all the people in order to make a decision ( $\mathrm{Li}$ et al. 2006). The usage of GIS technology in PPGIS projects has been limited due to its high start-up cost and complex user-interface (Kingston et al. 2000). However, Web-PPGIS is cost-effective and provides a higher degree of flexibility (Meng 2009). It is very efficient due to integration of interactive technologies for the provision and maintenance of GIS information (Chua and Wong 2002).

\subsubsection{The Shift to Electronic Participation}

The shift from traditional to e-participation happened at about the same time when GIS had been greatly explored as a potential data exploration and better decision-making tool for supporting public participation. Traditional desktop GIS, however, does not have "right-to-the-point" tools to support interactions among group members such as shared graphics, group modeling, and group consensus building. In addition, it lacks the capability to support collaborative spatial decision-making and public participation. To address this issue, efforts have been made under the umbrella term PPGIS to identify how GIS could support public participation for different application processes that require public participation (Craig et al. 2002; Sieber 2006).

With the advancement of information technology, especially the Internet and the World Wide Web, "the use of information and communication technologies to broaden and deepen political participation by enabling citizens to connect with one another and with their elected representatives" (Macintosh 2004) is becoming more prevalent. This new e-participation paradigm uses a number of tools and models to realize equivalent e-processes of the traditional participation activities, such as open houses, public hearings, opinion surveys, interviews and notification mails. For instance, voice conferences, e-newsletters, emails, online polling and feedback through forms or conducting sessions, videos chatting, online notifications, and discussion forums are new forms of traditional methods. While 
groupware tools, such as email, Electronic Meeting Systems (EMS), online audio/video conferencing, listserv, discussion forums and white-boarding, are among early adoptions of e-participation.

In addition, web 2.0 tools such as Wiki, blogging, and social networks have recently been explored. Under this new paradigm, traditional participation processes need to be re-examined, and some may need to be extended, if not redesigned.

Traditional and Web-based participatory processes of existing public participation practices are shown in Figure 2.5.

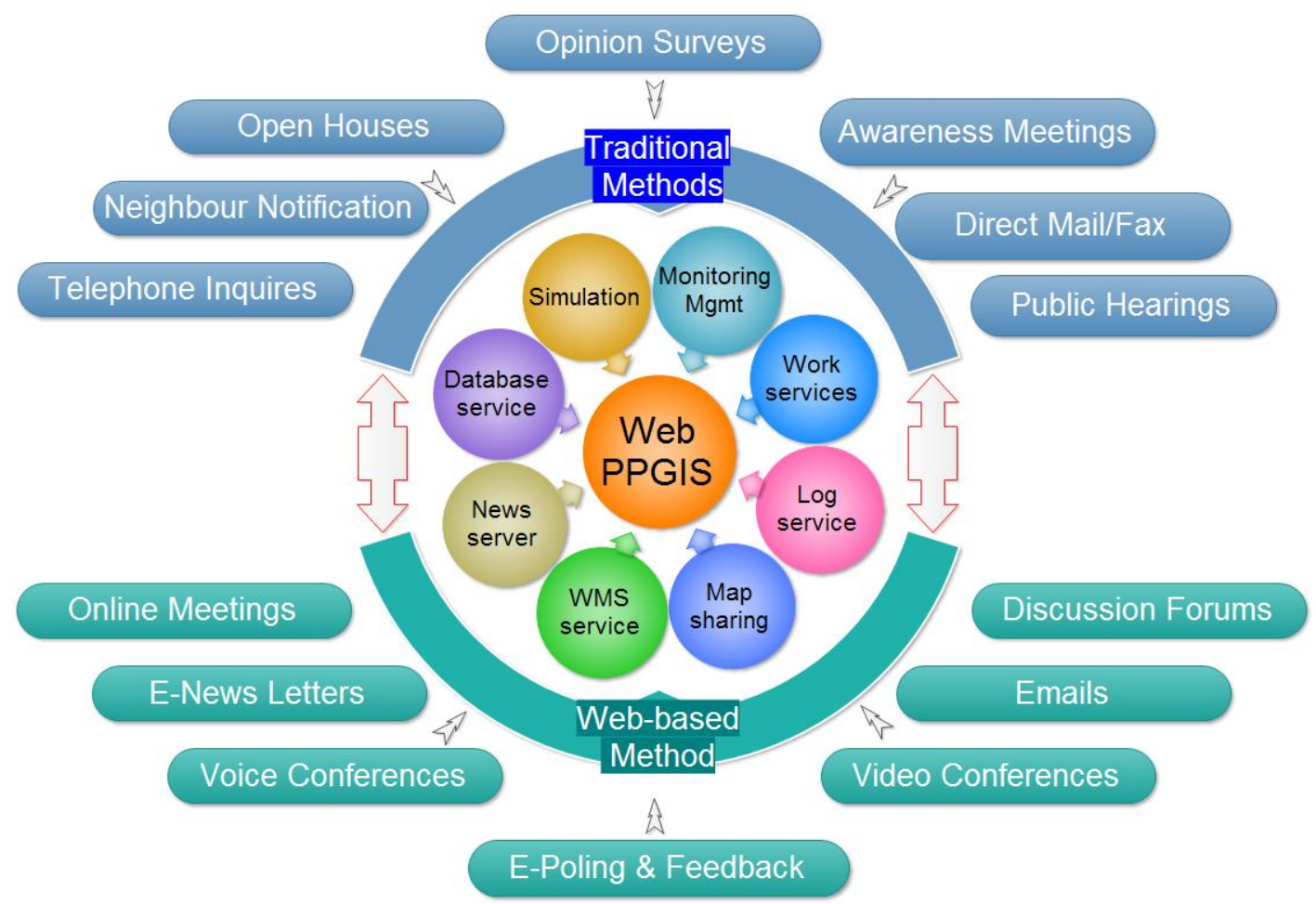

Figure 2.5: Traditional and Web-based Participatory Processes (Butt and Li 2014)

\subsubsection{Web-based Public Participation GIS}

Since 1992, a growing body of research has emerged in the use of geographic information systems, collaborative spatial decision-making systems, and web-based mapping to encourage and promote more informed public participation in government policy formulation and decision-making related to multiple projects (Obermeyer 1998). 
While early efforts focused on using GIS under controlled conditions to facilitate group decision-making (Jankowski and Nyerges 2001b and 2003), more recent developments (Kingston et al. 2000; Voss et al. 2004) have employed Web mapping with frequent feedback sessions (e.g., "geo-referenced" annotations) as a means of engaging citizens.

Laurini (2004) defines the web-based participatory GIS application such as web-PPGIS, "as a medium for exchanging information, ideas, and maps between all actors". Web-based participatory methods are more convenient (in the sense of time, location and information), and can be either unidirectional, bi-directional or multi-directional. The unidirectional approach has been known as the customary web participatory approach (Bryant and Wilcox 2006; Lemos 2006). In this approach, web pages are designed with only static contents, and therefore, support only one-to-many interactions. The bi-directional web participatory approach supports many-to-many interactions. Webbased collaborative GIS and GIS-enabled discussion forums are examples of bi-directional/multi-directional participatory approaches (Tang 2006). Furthermore, the bi/multi-directional participation requires a more collaborative and interactive participation process with the support of properly designed collaboration and userfriendly tools. Web-PPGIS research focuses on asynchronous use of maps in the participation processes. More recently, the importance of incorporating capabilities of real-time data sharing and exploration of spatially enabled alternatives and feedback have been discussed (MacEachren et al. 2001; Li et al. 2007). In brief, a key component of such a capability is to enable participants to collaborate on a shared map view in real-time while discussing the planning scenarios and their impact in detail. Moreover, when it is combined with other groupware tools such as chat, whiteboard, and audio/video conferencing, its benefits/advantages of enhancing effective public participation can be realized.

\subsubsection{Role of CSCW and Groupware in PPGIS}

CSCW began as an effort by technologists to learn from anyone who could help them to understand a group activity and how one could use tools and technology to support people in their work (Jones et al. 1997). The major capabilities these CSCW applications provide include: 1) informal and formal sharing and dissemination of several types of information, 2) collaborative viewing, editing, sharing and manipulating shared objects (in both synchronous and asynchronous mode), and 3) real-time communications (conferences and electronic meetings).

The CSCW technologies let people in remote areas interact with each other virtually by sharing data (files and documents) through information, voice and video links at the same time (Scientific 1990; Antunes et al. 2009; Abdalla and Li 2010; Baecker 1993). Numerous research efforts using Internet-based groupware technologies have been reported in the literature (e.g., Newton et al. 1995; Kao and Lin 1998; Rojas 1999, Li et al. 2006, Chang 2010, 
Butt and Li 2012, Butt and Li 2014). Nevertheless, they are also involved in the solution of different production problems related to manufacture, construction, and project management.

The term groupware is conventionally used to interpret those applications supporting the CSCW environment. It is the technical implementation of computer-supported collaborative work (Chang 2010). Interests in linking groupware with PPGIS have mainly focused on limited asynchronous collaboration, such as email and discussion forums. Rinner (1999, 2006a) and $\mathrm{Li}$ et al. (2007) realized the need to support collaborative discussions by introducing asynchronous-based geo-referenced mapping frameworks in which the discussion thread of each individual was linked with one or more elements on the map. These techniques offer a limited way of exploring spatial data or map information collaboratively, while this study focuses more on providing ways in which participants solve spatial problems together using synchronous based communicative platforms.

GIS applications are not well designed for collaborative activities (e.g., decision-making) (Armstrong 1994). A few GIS-based public participatory applications, with groupware and/or CSCW functionalities, have been developed using proprietary software approaches (e.g., PCI geo-conference). PCI geo-conference is an enterprise solution that allows multiple users in form of groups to share and view maps, terrain and imagery interactively over the Web, in real-time. Presently, efforts are made using open map services to develop simple map-sharing interfaces or applications. Several prototypes that facilitate anywhere (real-time) collaboration were designed and developed as a result of recent advancements in GIT, which support large spatial databases, groupware technologies and Web-based GIS (Churcher 1999; Jones et al. 1997; Dragicevic et al. 2004; Boulos et al. 2010). For instance, the Spatial Group Choice, a Spatial Decision Support System (SDSS), was developed by Jankowski et al. (1997) to support the CSCW environment. Similarly, the GroupARC was proposed and developed by Churcher (1996), which provides a tool for collaboratively viewing and annotating map/spatial data by geographically scattered people.

The prototype Real-time Environment Information Network and Analysis System (REINAS) designed and developed by Pang (1995), includes functionalities that are useful in the analysis of geospatial data. A Virtual Emergency Operations Center prototype was developed to provide a collaborative virtual environment that enables interactivity among participants while executing synchronous, script-driven tests and simulations (Fiedrich and Burghardt 2007). Chang (2010) developed the Synchronous collaborative 3D GIS to support synchronous collaboration efforts among geographically distributed people for enhancing collaborative decision-making. A GIS Design Server that allows the project team to better collaborate on a project through sharing the project's documents, spatial data and communications centralized in one secure location to work concurrently on a single seamless spatial database, was also developed (Longley et al. 2010). Furthermore, the GIS Design Server enables a 
collaborative spatial data warehousing solution. The Vehicle through Electronic Network (VENTEN) as a Disaster Geographical Information System serves as a central database and analysis tool for remotely sensed disaster data, and provides a communications channel to disaster management personnel. Thus, VENTEN provides effective application of disaster management using remote sensing and GIS technologies for disaster reduction, including route planning, distribution of rescue teams, quick response time and supporting the frontline relief operations (Boot et al. 2002).

\subsection{Examination of Selected Web-based Participatory GIS}

In order to further exam the details of existing PPGIS applications, five out of the ten reviewed online PPGIS applications, including Virtual Slaithwaite, Königslutter am Elm, Argumentation map, Map Chat, and GeoDF, were selected (see Appendix 8, Table I for the complete list of applications examined). They were further evaluated based on a number of evaluation criteria (see Table 2.1). The selection criteria are as follows: contribution to the field, relevance to the proposed research, and whether or not they are available online and functional with GIS mapping functions integrated for better participation and decision making. In addition to the general criteria, applications must also meet some specific requirements: 1) providing public access to the relevant tools and data and collect feedback to facilitate participation; 2) operational with GIS-based discussion functionalities at the time of evaluation; and 3) preferably Web-based user-friendly application (e.g., meet HCI criteria specified in Section 1.1.3).

Since evaluation and review are both important components of future enhancement and sustainable improvement of any PPGIS or non-PPGIS application, a literature review has been conducted on PPGIS technology in order to find out whether established criteria for the current evaluation purpose are available. Several researchers have already used different sets of criteria for evaluating online PPGIS applications. Therefore, the established criteria are somewhat similar to the criteria that were adopted by previous researchers, such as Chang (2010), Meng (2009), Zhao and Coleman (2006), Rinner (2006a), Li and Ma (2006) and Tang (2006). Furthermore, where applicable, the criteria developed by these researchers were adapted into the present evaluation.

The criteria listed in Table 2.2 were used for assessing whether a selected PPGIS application (see Section 2.3.1) could meet the communication requirements for public participation during municipality planning. The established criteria for this research study include the following: intended use, sharing of documents, exchange of information, real-time messaging with collaborative map-sharing support, effective communication of spatial context (i.e., discussion forum support), security implementation, collaborative decision support system, synchronous participation, GIS functionalities, public notification, and user-friendliness and interactivity of the user interface. 
These usability evaluation aspects (criteria) are discussed briefly in Table 2.2.

Table 2.2: Evaluation Criteria

\begin{tabular}{|c|c|}
\hline Criteria & Explanation \\
\hline Intended use & $\begin{array}{l}\text { The end users or one who are going to use at the end } \\
\text { to provide their feedback. }\end{array}$ \\
\hline of documents & Share, store, organize, and display discussions threads. \\
\hline $\begin{array}{lll}\begin{array}{l}\text { Exchanging } \\
\text { (participation) }\end{array} & \text { of information } \\
\end{array}$ & $\begin{array}{l}\text { Enable all participants to view ideas expressed by } \\
\text { other participants. } \\
\text { Support multi-way flow of information (i.e., from the } \\
\text { authority to the public; from the public to the } \\
\text { authority; and among the public). }\end{array}$ \\
\hline $\begin{array}{l}\text { Real-time messaging with } \\
\text { collaborative map sharing support }\end{array}$ & $\begin{array}{l}\text { aborative map sharing } \\
\text { functionalities }\end{array}$ \\
\hline $\begin{array}{l}\text { Effective communication of spatial } \\
\text { context with or without forum- } \\
\text { based support and multi-way } \\
\text { discussion forum support }\end{array}$ & $\begin{array}{l}\text { Asynchronous and synchronous communication } \\
\text { support of spatial context with or without initializing } \\
\text { forum-based discussion. Store and display the spatial } \\
\text { context of participants' views on a map along with the } \\
\text { corresponding expressions recorded by other media. } \\
\text { Provide structured organization of the integrated } \\
\text { discussion threads to facilitate understanding of } \\
\text { participants' worries. }\end{array}$ \\
\hline Secur & Login mechanism to protect abusive usage and spams \\
\hline $\begin{array}{l}\text { Collaborative decision support } \\
\text { system }\end{array}$ & $\begin{array}{l}\text { Collaborative decision support system to enhance } \\
\text { decision making }\end{array}$ \\
\hline Synchronous participation & $\begin{array}{l}\text { chronous participation using map and } \\
\text { a }\end{array}$ \\
\hline GIS functi & $\begin{array}{l}\text { functions and analysis, i.e., buffer } \\
\text { is }\end{array}$ \\
\hline Public Notification & a, i.e., e-newsletter, and auto email. \\
\hline $\begin{array}{l}\text { User friendliness and i } \\
\text { of the user interface }\end{array}$ & $\begin{array}{l}\text { Interactivity of the user interface by applying HCI set } \\
\text { of rules. }\end{array}$ \\
\hline
\end{tabular}

In brief, the application should be functional with GIS-based mapping/argumentation functionalities for finding better comparative results because some of these applications are being enhanced on an ongoing basis (Butt and $\mathrm{Li}$ 2014). Bevan (1995) stated that comparing usability of systems that have similar main functions should be considered a part of successful implementation of the system design. The examination of the existing PPGIS applications allowed us to learn what the limitations of the current practice and application frameworks. In addition, the selection and evaluation of the following PPGIS applications help to decide what kind of GIS mapping functionalities, as well as participatory approaches, should be included in the research prototype for the expected 
outputs. The established criteria (as shown in Table 2.2) were used for producing the review and comparative results for assessing some selected online PPGIS applications (see Section 2.3.1 and 2.3.2).

\subsubsection{Review of Selected Collaborative Participatory GIS}

\section{i) Virtual Slaithwaite}

The Virtual Slaithwaite project developed as PPGIS to identify the user's views regarding how they would like their municipality to be developed in the future. It was initiated by the Leeds School of Geography and funded by the British government. The purpose of this system was to take valuable suggestions from the citizens. Figure 2.6 shows the interface of the digital version of the system, which provides the following functionalities to the public: 1) exchange of spatial information; 2) map navigation tools, and 3) a feedback component to submit general comments.

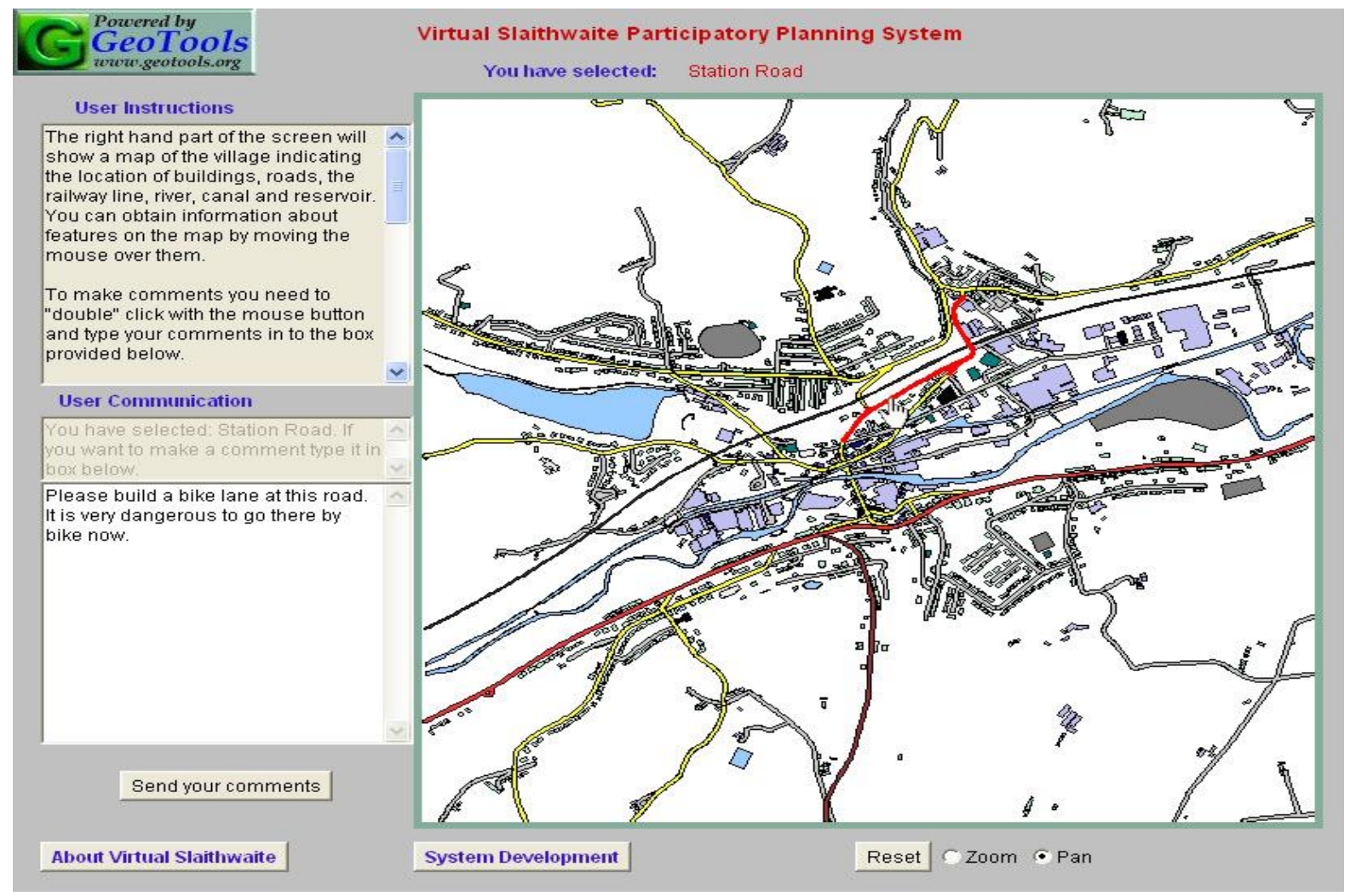

Figure 2.6: Virtual Slaithwaite Project (last accessed in January 2014: http://www.ccg.leeds.ac.uk/slaithwaite/) 
The system was designed to accumulate citizens' ideas and comments. The users had a window to share their thoughts on any point or location on the map using the 'add text' tool. These comments are represented by small dots and can be viewed by any user (Evans et al. 1999). The user can use some other GIS mapping tools' functionalities. For example, map navigation can be performed with zoom and pan tools. Furthermore, the user can also perform a spatial query to retrieve the spatial data of the village (Slaithwaite). Based on Evan et al. (1999) and Carver (2001), the Graphical User Interface (GUI) of the system is user-friendly.

There were also a few drawbacks. First, the system performance during the display of spatial data was a little slow due to the absence of a map server. Moreover, maps are generated with the help of PERL scripting language modules, which is not considered a generic and efficient solution. Second, the comments were less organized or structured. For example, the public cannot reply further to any previously created topics/comments. In brief, the GIS-based system does not provide options for feedback on any topic so discussion among the multiple stakeholders.

\section{ii) Interactive Landscape Plan Königslutter am Elm}

The interactive landscape planning system "Königslutter am Elm" is an online public participation geographic information system sponsored by the Federal Agency for Nature Protection (FANP, Lower Saxony, Germany; http://thuja.land.uni-hannover.de/). This online PPGIS provides access to the stakeholders with several types of information regarding landscape planning for the area Königslutter am Elm. It was developed to complement the traditional public participatory approach.

The intended use of this PPGIS application was to facilitate citizen participation by providing a GIS-enabled discussion platform. The online PPGIS platform in Königslutter provides citizens email communication to the project team, a map annotation module, a discussion forum, and feedback forms for the interactive maps (WarrenKretzschmar and Tiedtke 2005). Although this GIS-based application has almost the same GIS mapping functions, as the previously discussed system, it provides an effective solution for small budget municipalities due to its cost-

effective use of OSS technologies. The University of Minnesota (UMN) Map Server is used to generate maps and simple images as map layers. In addition, the MySQL database server is used to handle textual comments and spatial data. Both OSS technologies are widely used nowadays. 
The interactive interface of the system is shown in Figure 2.7.

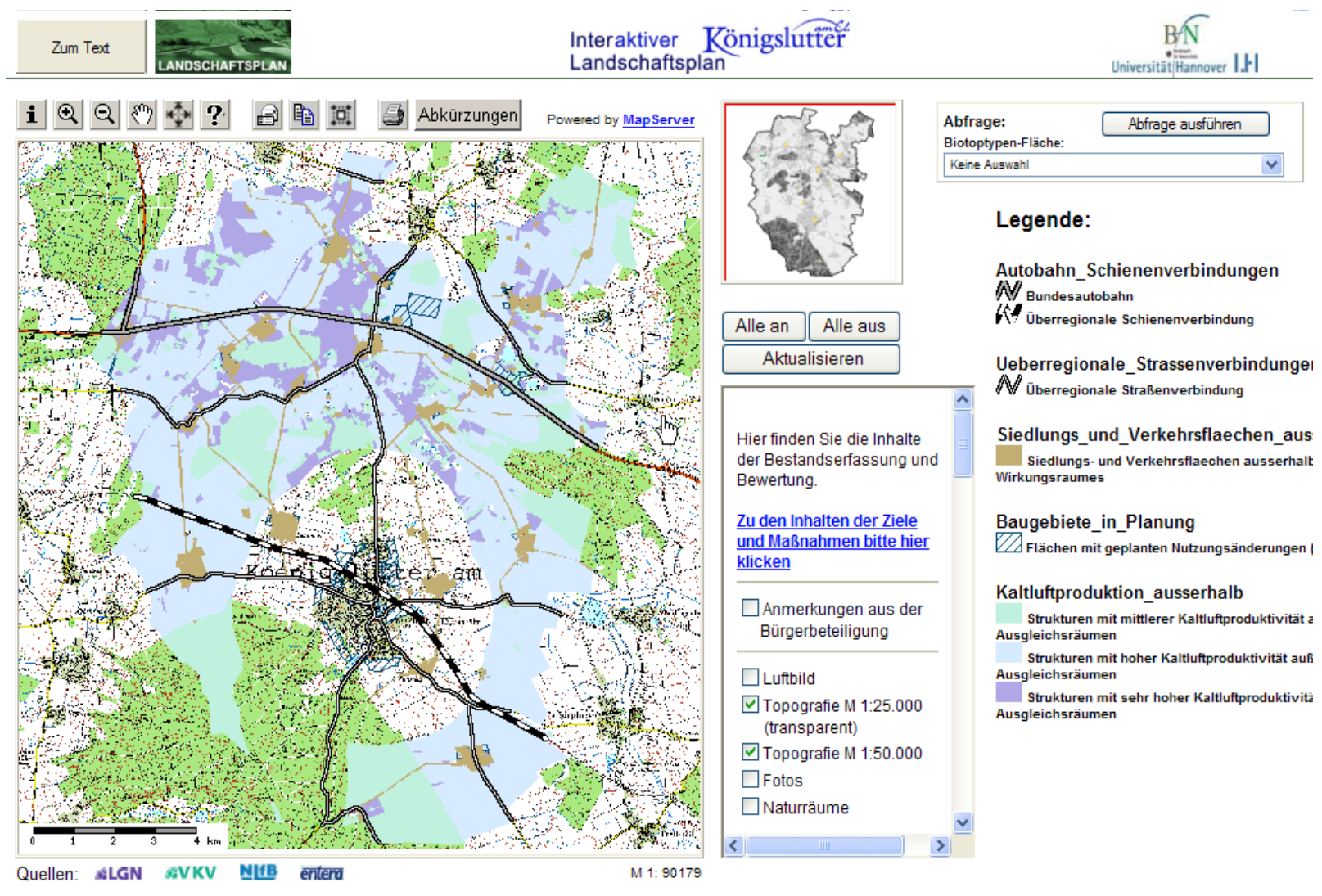

Figure 2.7: Landscape Plan Königslutter am Elm (last accessed in January 2014: http://www.koenigslutter.de/)

Although the Königslutter am Elm system is more economical compared to the Virtual Slaithwaite system, the oneto-many participatory approach still makes this system less effective. Information is streaming between the public and the authorities, but there is no support of multi-user collaborative discussion forum functionality.

This PPGIS application implements a basic argumentation method that has been used in public participation procedures, allowing the public to place annotations and comments on a map. Annotations are placed on the corresponding real-world geographic locations/geographic reference objects on a map. Although the Königslutter am Elm system enables the public to comment on map reference objects, it does not support online forum style discussions. In addition, one annotation can only refer to one geographic reference feature of a map. It also is not 
possible to make graphic reference objects on the base layer of a map (Keßler 2004; Rinner et al. 2008). No doubt, with a few more functions (i.e., support of multi-way discussion forum) would make this system more effective.

\section{iii) Argumentation Map}

Argumentation map was introduced and developed by Rinner $(1999,2001)$ for discussions based on geospatial data through cartographic visualization and query functionality. The intended use of this PPGIS prototype was to facilitate both end users and content providers.

This GIS-based application has a user-friendly interface and public participation methods. For example, its argumentation model supports a multi-way collaborative discussion among its stakeholders, graphics objects, geographic elements, and argumentation elements. The argumentation map is often structured into discussions with contributions by individual participants responding. Argumentation map supports many-to-many relationships among its elements: argumentation elements (participant comments), and geographic map objects/features (point, line and polygon). It supports multi-discussion, which means an argumentation element can reference several geographic features on maps and a geographic map feature can be referenced by several argumentation elements (Rinner 2006a). In brief, the argumentation map provides a link between a mapping component and a discussion tool. This prototype was designed using open-source software (OOS) technologies and APIs. The Java Applet was used to implement most of its functionalities, which make effective use of both the discussion forum and the map. The server-side component of the prototype is developed using Java Servlets. Maps were generated using the GeoTools Lite Libraries and an open-source Java API. Users can search different discussion contributions using a keyword. 
The interactive interface of the prototype is shown in Figure 2.8.

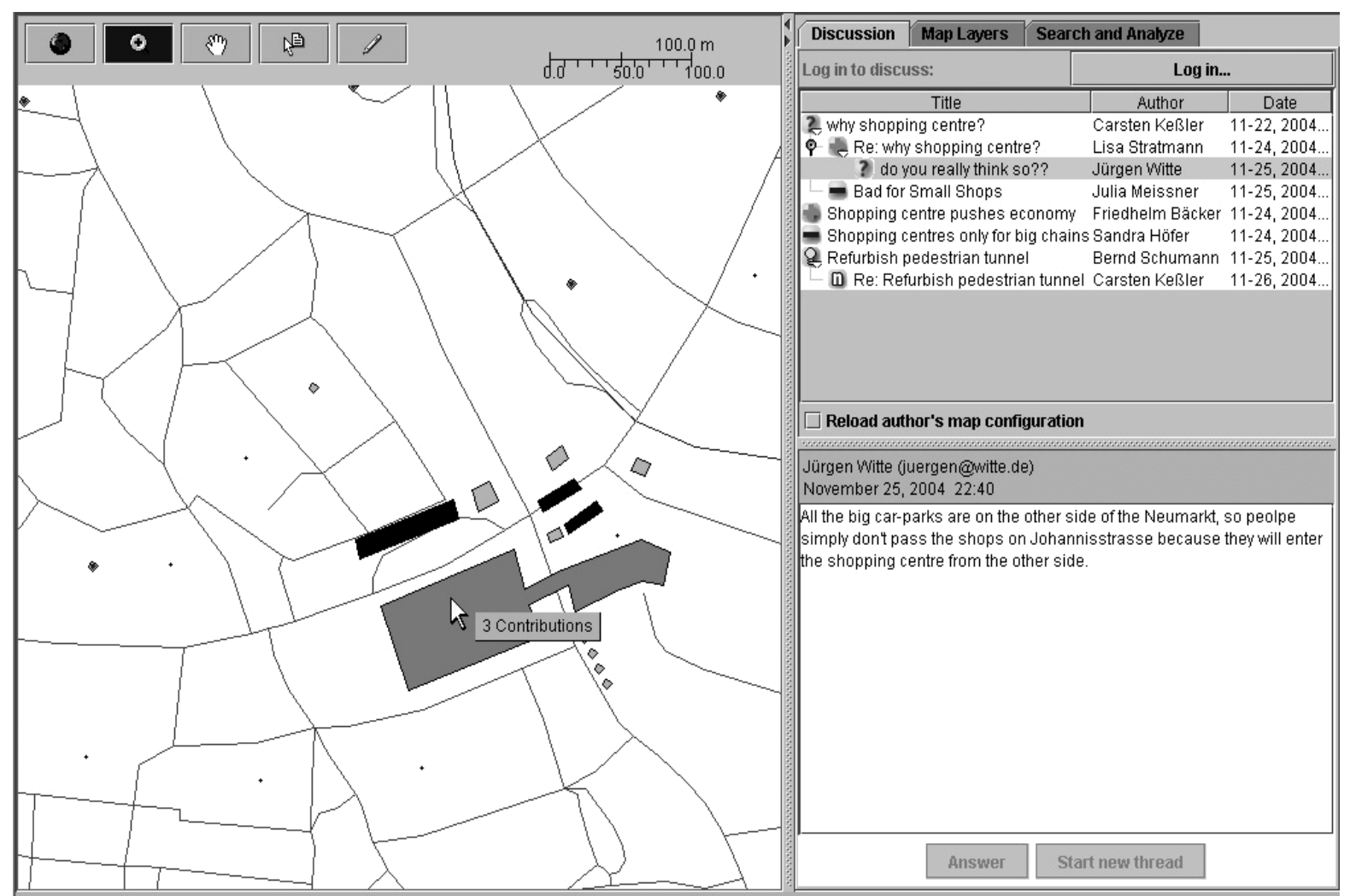

Figure 2.8: Argumentation Maps (last accessed URL: http://www.agile-

online.org/Conference_Paper/CDs/agile_2005/papers/66_Carsten\%20Kessler.pdf)

This system is cost effective because of its OSS-based framework implementation using Java APIs and Servlets programming, especially for smaller municipalities who have lower budgets. The system performance may be improved for generating maps in the presence of the MapServer/GeoServer, which has a built-in map cache service that is used for producing maps quickly as compared to GeoTools libraries. This innovation would also help to improve response time to the spatial queries.

\section{iv) $\underline{G e o D F}$}

The GIS-enabled Online Discussion Forum (GeoDF) is an online public participation GIS that was introduced and developed by Tang (2006). Tang (2006) used the concept of multi-way collaborative discussion similar to Argumentation Map (Rinner 1999) discussed earlier. 
The intended use of this GeoDF prototype was to facilitate the public participation for collaborative decisionmaking. This GeoDF application has forum style discussion support for public participation, which is developed using OSS-based PHP Built-in Board (PHPBB). It is supported by geographic map features, such as sketches, zoom, pan, and graphic annotations with text, and shows areas of the hottest discussions to support the full expression of users' and experts views. The PHPBB is a flat-forum bulletin board solution which was integrated with the commercially available GIS architecture (ESRI ArcIMS) to enhance the GIS mapping functionalities of the GeoDF. Other than using different technologies and programming languages, the Argumentation map and GeoDF both follow the same model of forum style discussion support during the development of their collaborative participation modules.

The main interface of a GIS-enabled online discussion forum is shown in Figure 2.9.

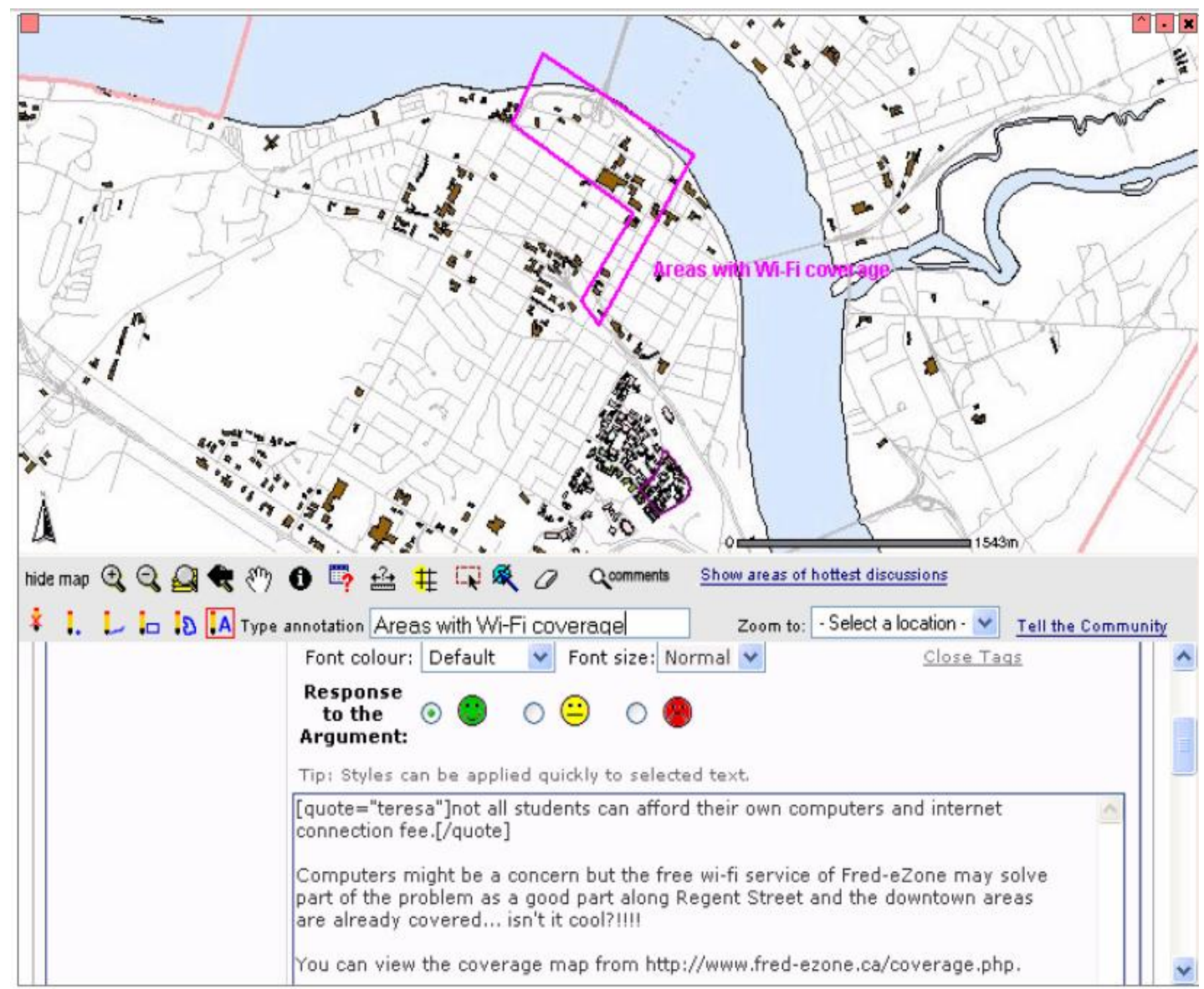

Figure 2.9: GIS-enabled Online Forum (http://www.i-cherubini.it/mauro/blog/2007/06/26/) 
GeoDF is considered functional with an asynchronous public participatory approach because of the implementation of multi-way collaborative discussion through which both the textual and spatial information is flowing between the map elements, argumentation elements and multiple stakeholders. Increasing numbers of participants may create some issues related to the readability and usage of map objects. Providing a functionality of 'map bookmarking' to save multiple map extents (map areas) with the discussion for each municipal project may resolve this issue. In addition, more future work may be required with real-time multi-participant discussion support that may enhance participation methods of PPGIS. Real-time multi-participant discussion support can be defined as one map element (i.e., area of study) related to any municipal planning project can be discussed by many participants on real-time basis at the same time from distant locations.

\section{v) MapChat}

MapChat is a Web-based application that consists of real-time protocols for sharing maps and imagery among geographically distant participants and/or decision makers (originally designed by Hall and Leahy 2006). Participants can discuss freely, in question and answer format, using its real-time chatting (geo-chatting) feature. Workspace consists of different vector layers. This workspace option is such a beneficial and sophisticated tool because it accomplishes urgent response and quick actions in municipal planning, management and emergency scenarios. MapChat facilitates drawing and other mapping tools for participants to point out the deficiencies/improvements related to ongoing municipal projects, which are also commonly shared on the main map interface. An individual can give his or her comments and suggestions for better decision-making in order to minimize or overcome the effects of an event. For example, in emergency management related activities, it may provide an efficient platform for Non-Government Organizations (NGOs) and other emergency relief teams to gather rapid response and useful information related to the occurrence of relevant incidents that involved multiple stakeholders. Moreover, this tool may provide concrete foundations to decision authorities in order to make future strategies and handle any situations related to municipal planning and emergency management. MapChat, as its name suggests, means one is actively chatting on a map. One of the main features of MapChat is to provide georeference based chatting in which all map-based discussions are geo-tagged to real-world spatial objects/features. It is also a PPGIS that has the ability to conduct online discussions over a map between different participants.

Participants in MapChat sessions use lightweight, easy-to-deploy client software to take part in collaborative discussions based on asynchronized map views. Using this OSS-based tool, they are in effect performing "geocollaboration", in the form of live, map-based Internet conferences. 
The intended use of the MapChat application is to arrange meeting sessions among people from distant locations in a very efficient way (see Figure 2.10).

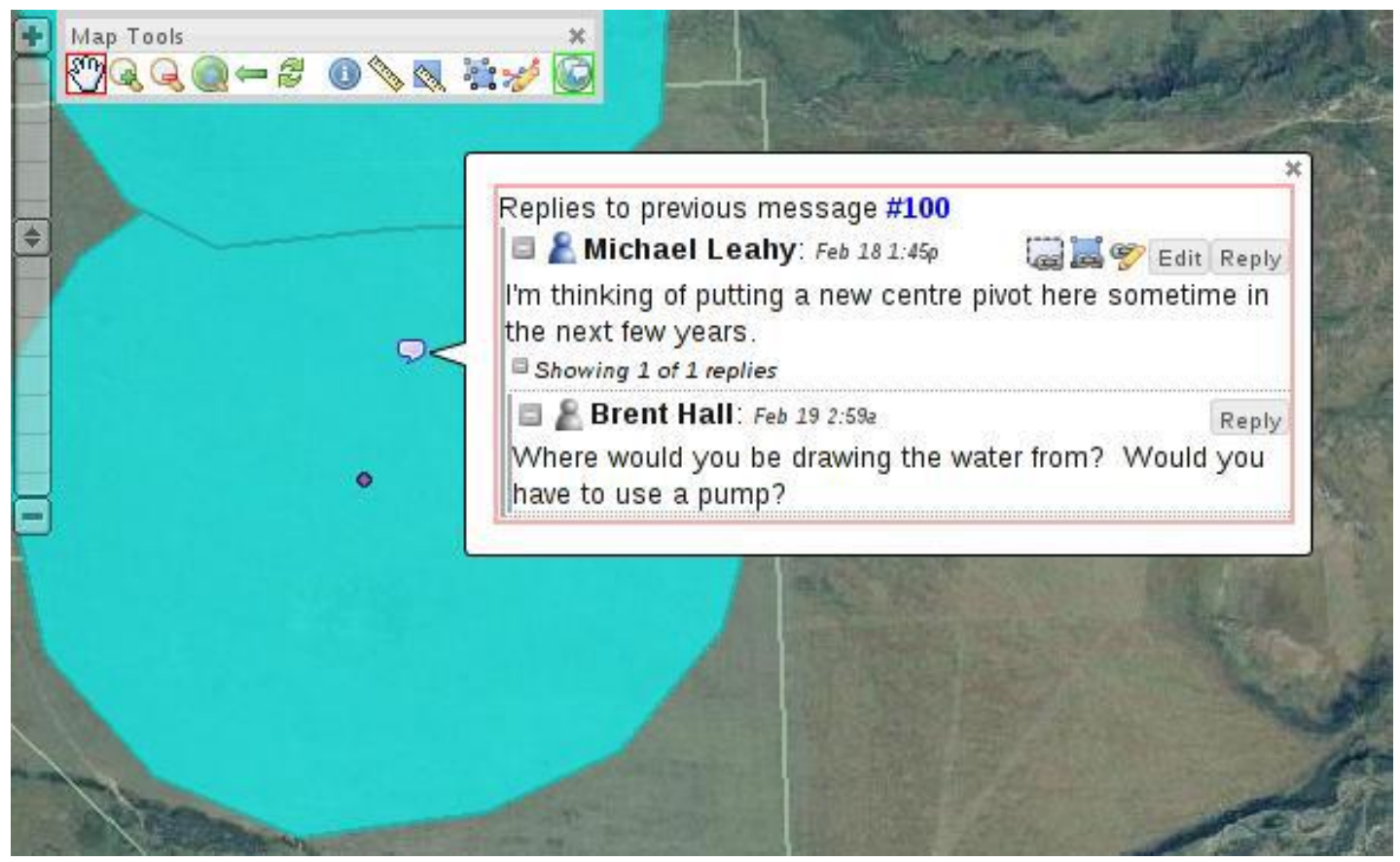

Figure 2.10: GIS-enabled Online MapChat (URL: $\underline{\text { http://www.mapchat.ca) }}$

Moreover, the intended use of this PPGIS application was to facilitate public participation during municipal planning and management-related activities. This MapChat application has multi-way discussion forum support with its real-time chatting feature. Similar to the GeoDF, in case that a large number of participants participate in the same area of study, MapChat's readability may be affected while marking an area with multiple graphic reference objects. With the integration of a real-time map-sharing mechanism, groupware and CSCW support, this prototype can be made more efficient.

\subsubsection{Comparison of the Selected Online Collaborative Participatory GIS Applications}

The evaluation of the existing PPGIS applications helped researchers to identify limitations of the current practices and application frameworks. It also suggested communication channels/functionalities and participatory approaches in the research work. As discussed early in Section 2.3, the evaluation criteria were based on a number of principles: intended use, sharing of documents, exchanging of ideas, real-time messaging with collaborative map support 
system using GIS functionalities, effective communication of spatial context with or without forum-based support, security implementation, collaborative decision-making culture, and interactivity of the user's interface.

The purpose of designing GIS-based systems is to enhance public participatory approaches in the planning and development-related discussions. The intended use of these systems was to facilitate public participation in an efficient and effective way. Except for Virtual Slaithwaite, which supports vector data, other applications facilitate raster image maps. Furthermore, in interactive landscape and GIS-enabled online discussion forum (GeoDF) maps were produced by using map servers (ESRI ArcIMS and University of Minnesota Map-Server), whereas in Virtual Slaithwaite and Argumentation prototype maps were produced by using PERL scripts and Java API's. A comparison chart was created based on the above evaluation criteria and presented in Table 2.3.

Table 2.3: Comparison of Selected PPGIS Applications (Butt and Li 2014)

\begin{tabular}{|c|c|c|c|c|c|c|}
\hline $\begin{array}{l}\text { Features/ } \\
\text { PPGIS }\end{array}$ & Function List & $\begin{array}{l}\text { Virtual } \\
\text { Slatith }\end{array}$ & MapChat & $\begin{array}{l}\text { Interactive } \\
\text { Landscape }\end{array}$ & $\begin{array}{l}\text { Argoo } \\
\text { Map }\end{array}$ & Geo DF \\
\hline \multicolumn{7}{|c|}{ Info Centre/Management } \\
\hline & $\begin{array}{l}\text { Document } \\
\text { Transfer: }\end{array}$ & 0 & $\mathrm{O}$ & $\mathrm{O}$ & 0 & $\mathrm{O}$ \\
\hline & $\begin{array}{c}\text { Search } \\
\text { Document: }\end{array}$ & 0 & 0 & 0 & $\begin{array}{c} \\
\text { Search } \\
\text { Discussion } \\
\text { Contribution }\end{array}$ & $\begin{array}{l}0 \\
\text { Manually } \\
\text { Search }\end{array}$ \\
\hline & $\begin{array}{c}\text { Meeting } \\
\text { Documents: } \\
\text { By laws, Meeting } \\
\text { Minutes, Meeting } \\
\text { Location Finder } \\
\text { using Map, } \\
\text { Agendas etc. }\end{array}$ & 0 & 0 & 0 & 0 & $\begin{array}{l}\quad 0 \\
\text { Consulted } \\
\text { Document } \\
\text { (PDF) } \\
\text { Supported } \\
\text { data }\end{array}$ \\
\hline & $\begin{array}{c}\text { GIS Data } \\
\text { Download: }\end{array}$ & 0 & 0 & 0 & 0 & 0 \\
\hline \multicolumn{7}{|c|}{ Communication/Information Delivery } \\
\hline & Email: & 0 & 0 & O & 0 & 0 \\
\hline & Map Attachment: & 0 & 0 & O & 0 & 0 \\
\hline
\end{tabular}




\begin{tabular}{|c|c|c|c|c|c|c|}
\hline & & & & Print PDF & & \\
\hline & Newsletter: & 0 & $\mathrm{O}$ & 0 & 0 & $\mathrm{O}$ \\
\hline & Feedback Polls: & $\mathrm{O}$ & $\mathrm{O}$ & $\mathrm{O}$ & 0 & 0 \\
\hline & $\begin{array}{l}\text { Discussion } \\
\text { Forum: }\end{array}$ & $\begin{array}{c} \\
\text { Initiate } \\
\text { Discussion }\end{array}$ & 0 & $\begin{array}{c}0 \\
\text { Initiate } \\
\text { Discussion }\end{array}$ & 0 & O \\
\hline & $\begin{array}{c}\text { View Participants } \\
\text { Feedback: }\end{array}$ & O & O & O & O & O \\
\hline \multicolumn{7}{|c|}{ Synchronous Participation \& Collaborating Decision-making } \\
\hline & $\begin{array}{c}\text { Real-time } \\
\text { Messaging: } \\
\text { Meeting } \\
\text { Attendees \& } \\
\text { Facilitator }\end{array}$ & 0 & O & $\mathrm{O}$ & 0 & 0 \\
\hline & $\begin{array}{c}\text { Real-time } \\
\text { Map-sharing: }\end{array}$ & 0 & $\mathrm{O}$ & 0 & 0 & 0 \\
\hline & $\begin{array}{c}\text { Video } \\
\text { Conferencing: }\end{array}$ & 0 & $\mathrm{O}$ & 0 & 0 & 0 \\
\hline & Web Meeting: & 0 & 0 & 0 & 0 & 0 \\
\hline & $\begin{array}{l}\text { Video Chatting } \\
\text { (IRC): }\end{array}$ & 0 & 0 & 0 & 0 & 0 \\
\hline & $\begin{array}{l}\text { E-White } \\
\text { Boarding: }\end{array}$ & 0 & 0 & 0 & 0 & 0 \\
\hline & Screen Sharing: & 0 & 0 & 0 & 0 & 0 \\
\hline \multicolumn{7}{|c|}{ Notification/ Scheduling } \\
\hline & $\begin{array}{c}\text { Meeting Event } \\
\text { Handling: }\end{array}$ & 0 & 0 & 0 & 0 & 0 \\
\hline & $\begin{array}{c}\text { Event } \\
\text { Notification: }\end{array}$ & 0 & 0 & 0 & 0 & 0 \\
\hline & Calendaring: & $\overline{0}$ & 0 & 0 & 0 & 0 \\
\hline & $\begin{array}{c}\text { Share } \\
\text { Scheduling: }\end{array}$ & 0 & 0 & 0 & 0 & 0 \\
\hline \multicolumn{7}{|c|}{ GIS Functionalities } \\
\hline & Exploring Spatial & 0 & 0 & 0 & 0 & 0 \\
\hline
\end{tabular}




\begin{tabular}{|c|c|c|c|c|c|c|}
\hline & $\begin{array}{l}\text { Data (with Forum } \\
\text { support): }\end{array}$ & $\begin{array}{c}\text { Not Fully } \\
\text { Supported } \\
\text { Forum activities } \\
\text { i.e., Starting } \\
\text { Topics, Forums } \\
\text { etc. }\end{array}$ & & $\begin{array}{c}\text { Not Fully } \\
\text { Supported } \\
\text { Forum } \\
\text { activities i.e., } \\
\text { Starting } \\
\text { Topics, } \\
\text { Forums etc. }\end{array}$ & & \\
\hline & $\begin{array}{c}\text { Exploring Spatial } \\
\text { Data } \\
\text { (Non- Forum): }\end{array}$ & 0 & 0 & 0 & 0 & 0 \\
\hline & $\begin{array}{l}\text { Organization of } \\
\text { Map data: using } \\
\text { Spatial Database }\end{array}$ & $\mathrm{O}$ & 0 & -- & -- & $\begin{array}{c}\text { O } \\
\text { Geo- } \\
\text { database \& } \\
\text { MySQL } \\
\text { used for } \\
\text { Textual } \\
\text { Data }\end{array}$ \\
\hline & Map Navigation: & 0 & 0 & 0 & 0 & 0 \\
\hline & Map Printing: & 0 & 0 & 0 & -- & \\
\hline & Spatial Query: & 0 & 0 & 0 & -- & 0 \\
\hline & $\begin{array}{c}\text { Commenting } \\
\text { Tools: Sketches, } \\
\text { Annotation }\end{array}$ & 0 & 0 & 0 & $\begin{array}{c} \\
\text { Annotation }\end{array}$ & 0 \\
\hline \multicolumn{7}{|c|}{ Security Implementation } \\
\hline & $\begin{array}{c}\text { Login } \\
\text { Authentication: }\end{array}$ & 0 & 0 & 0 & -- & 0 \\
\hline & Forum Security: & $\mathrm{O}$ & 0 & $\mathrm{O}$ & -- & -- \\
\hline & $\begin{array}{l}\text { User Rights \& } \\
\text { Privileges: }\end{array}$ & 0 & 0 & 0 & $\overline{--}$ & -- \\
\hline \multicolumn{7}{|c|}{ User Friendliness } \\
\hline & $\begin{array}{c}\mathrm{HCI} \\
\text { Implementation: } \\
\text { Strive of } \\
\text { Consistency }\end{array}$ & 0 & 0 & 0 & 0 & 0 \\
\hline
\end{tabular}




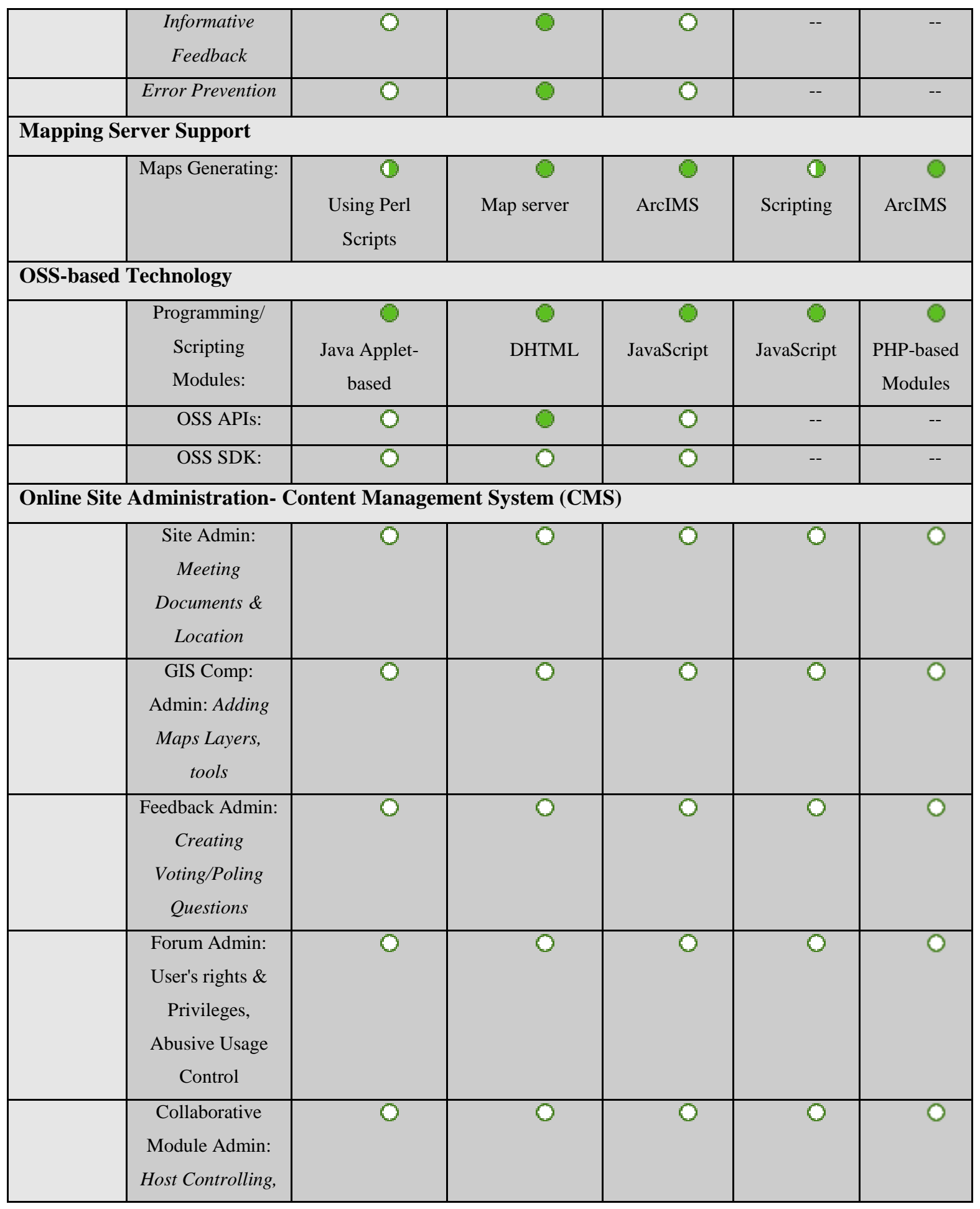




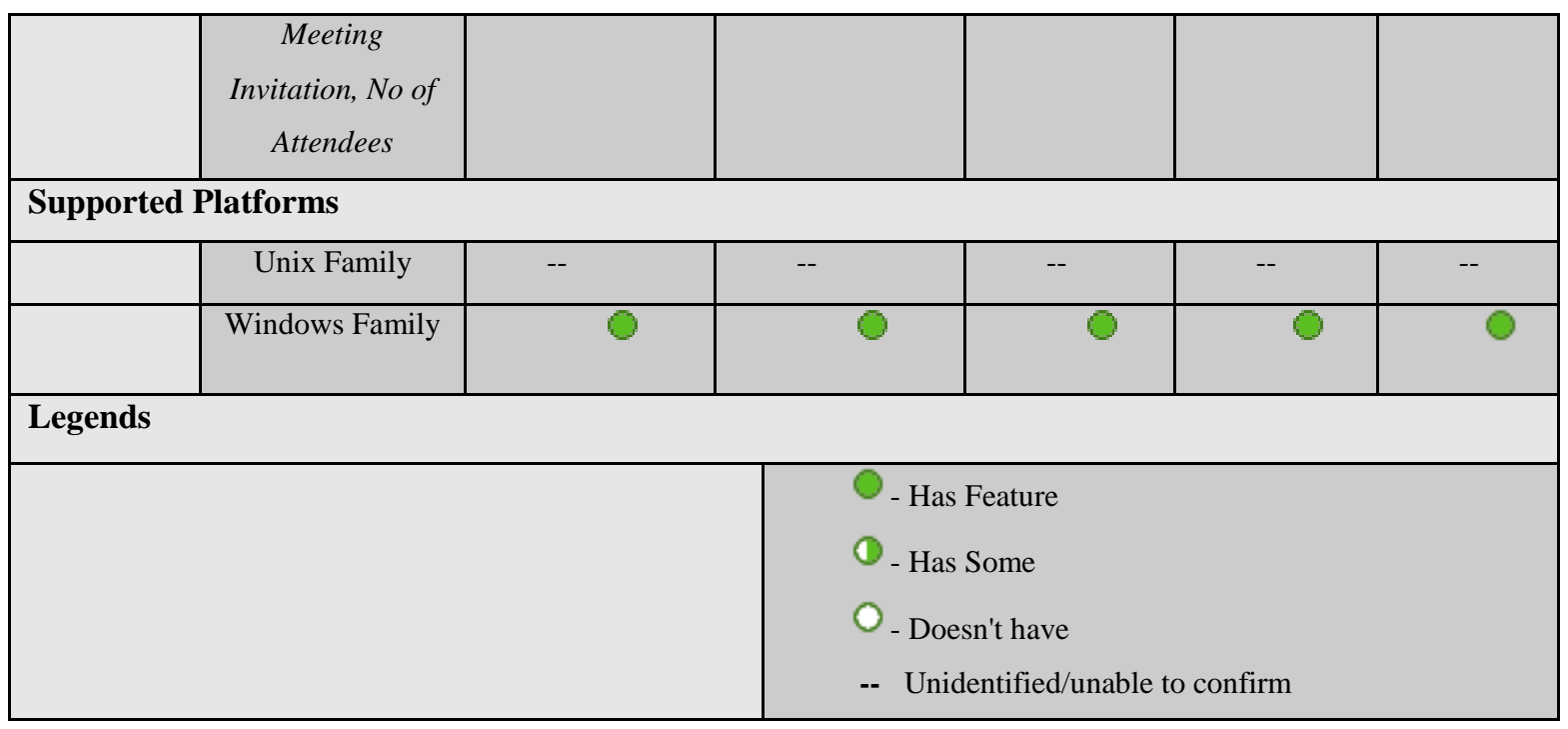

The systems discussed above do not support multi-way discussion forums for public participation. Only GeoDF, MapChat, and Argumentation Map have forum-style functionalities for the exchanging of ideas. Both MapChat and Argumentation map prototypes were designed using open source software technologies; and therefore, provide a relatively cost-effective solution as compared to others (Tang 2006). Mapchat has a built-in geo-chatting feature, which provides participants the opportunity to chat against specific graphic features drawn on the map for municipal planning purposes. All system interfaces seem to be user-friendly to some extent. For example, the main interfaces of Argumentation map and Virtual Slaitwaite were designed using Java applets programming, while other system's interfaces were designed using Dynamic Hyper Text Mark-up Language (DHTML) and Java Scripts. Some of the features which may be required and have not been implemented in any of the systems are: 1) synchronous participation and collaborating decision-making, 2) notification/meeting scheduling, and 3) online project data/contents management.

\subsubsection{Summary of Closely-related Research Models}

The three research models discussed below were considered relevant to the present research study. The argumentation model was introduced by Rinner (1999), in which he introduces argumentation maps as an objectoriented model for geographically referenced discussions. 
As shown in Figure 2.11, it describes the relationships between an argumentation element (discussion), a geographic reference object (map feature) and user defined graphic reference objects (sketches) (Rinner 2006b).

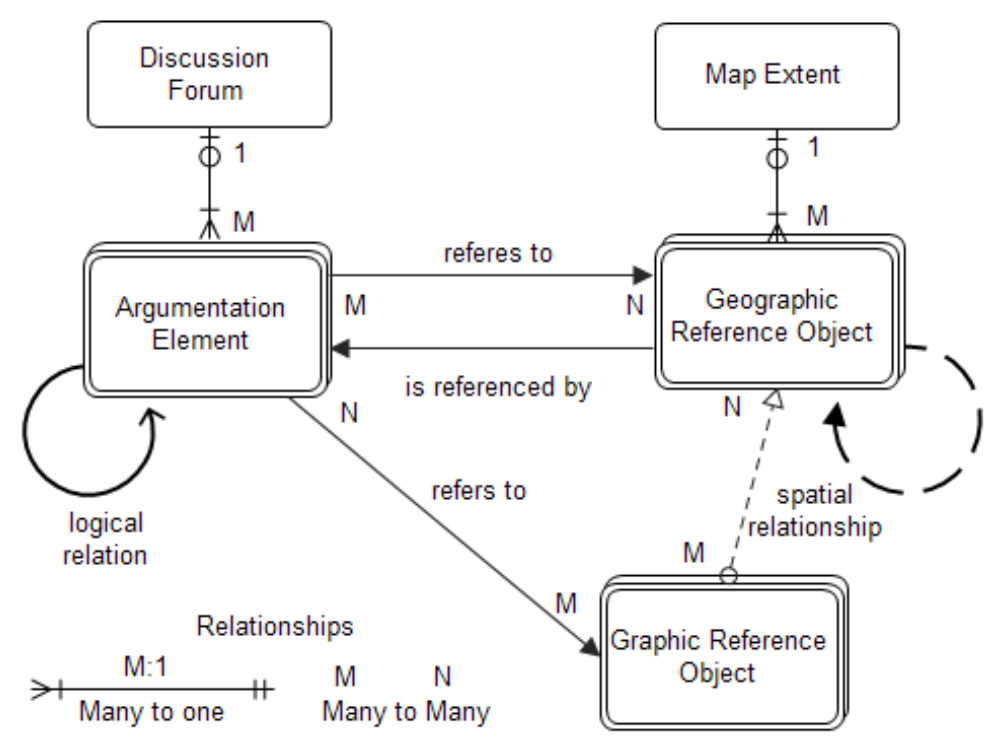

Figure 2.11: Modified Argumentation Map Model (Rinner 2006)

The argumentation model, consisting of object classes, can support many-to-many relationships. For instance, a geographic object can reference several argumentation elements and an argumentation element can be referenced by several geographic objects. Moreover, as shown in Figure 2.11, the objects have self-relationships to other objects of the same class. For instance, geographic reference class objects may have spatial relations to each other, and argumentation elements class objects may have logical relations to each other (Rinner 2006a). The argumentation model provides a prototype for participatory planning and management scenarios with a special focus on the use of standards to confirm interoperability (Keßler et al. 2005). The discussion component was developed using open source programming languages (i.e., JavaScript and Java applet). The map component relies on open-source Java API for maps (i.e., GeoTools libraries).

Similar models were introduced and adopted by Tang (2006) and Hall and Leahy (2006) but different technologies were used to develop the research prototype GeoDF and MapChat. As discussed in the previous section, Tang integrated PHPBB with commercial-based ESRI ArcIMS to develop a GeoDF prototype. Figure 2.12, developed based on Tang (2006), shows different components of GeoDF model. 


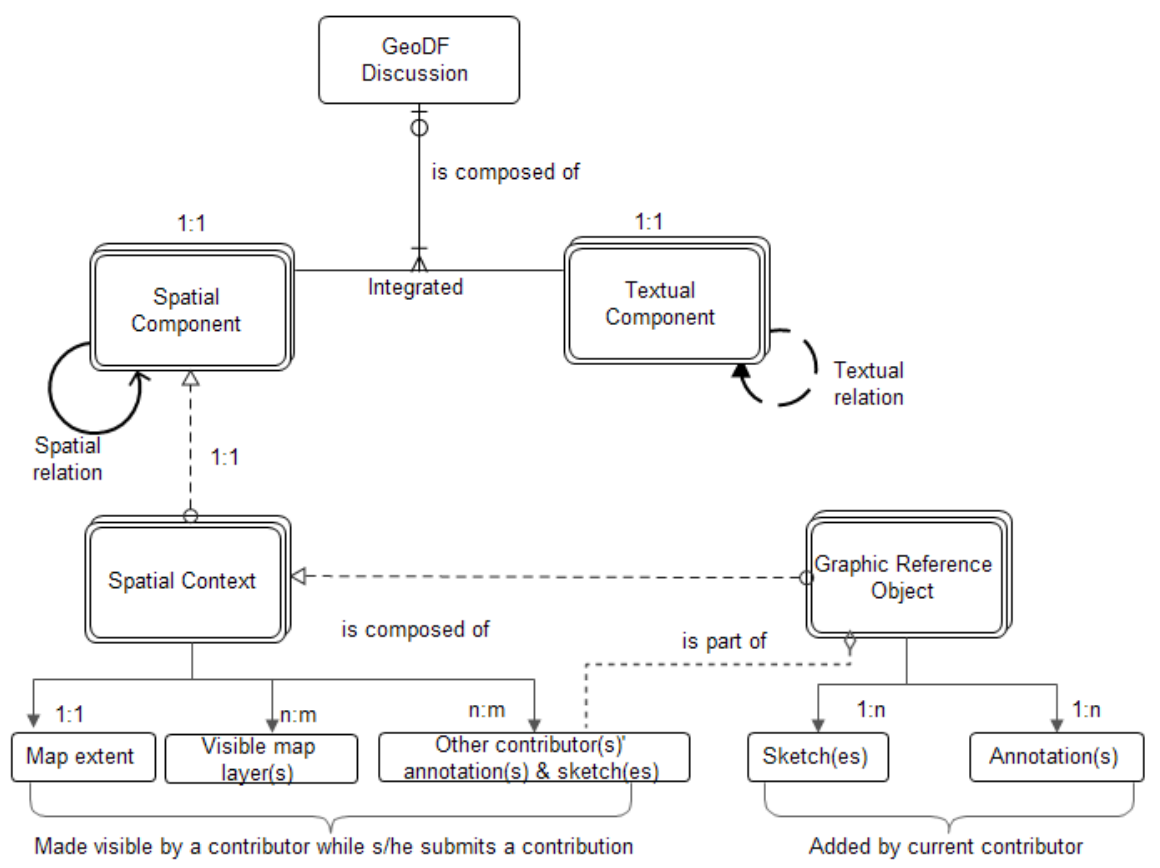

Figure 2.12: Modified Argumentation Map Model for GeoDF

Each discussion is composed of two major components (i.e., the spatial and textual components). The textual component refers to a contributor's understanding expressed as text. The spatial context, which is a part of spatial component, is a combined term for the geographic features, map extent, location, and spatial relationships embedded in GeoDF discussion threads. The discussion threads are the thoughts, views, or feedback submitted by a participant via GeoDF. In other words, the spatial context is mainly composed of graphic reference objects (i.e., sketch(es), annotation(s), and other contributor(s)' sketches) and annotations along with other two spatial elements, namely the map extent, visible map layer(s) (Tang 2006).

The MapChat argumentation model employs similar classes and objects for spatial and textual relationship in comparison with the previously discussed models. A new real-time map discussion class was introduced in this model, which provides the functionality of real-time chatting in connection with each graphic reference object. The MapChat argumentation model contributes to an open-source application infrastructure. For instance, it employs open standards in terms of the overall system specification. It uses JavaScript and PHP-based open source coding, and it uses a flexible architecture to provide the integration of other modular tools (Hall and Leahy 2006). 
Figure 2.13, developed based on Hall and Leahy (2006), shows different components of MapChat application.

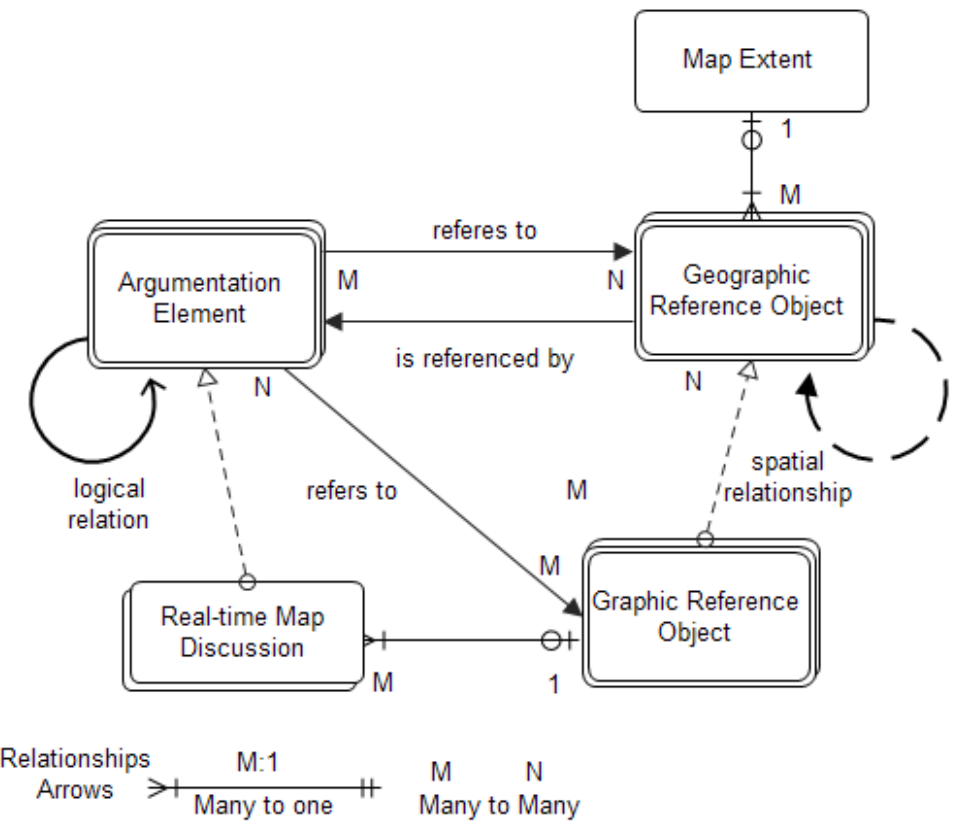

Figure 2.13: Argumentation Map Model for MapChat

These models share common features: introducing open standard-based object models, sharing the same map extent during discussion, asynchronous participatory approach for map-based discussion and making many-to-many relationships among its elements. They all provide structured discussions, about different map features and/or geographic/graphic reference objects, among several geographically distant meeting participants to facilitate an asynchronous spatial data-sharing approach. However, with the asynchronous spatial data-sharing approach, it is not possible to identify an argumentation element or refer to the real-world object simultaneously among different participants. The MapChat has real-time chatting discussion functionality, which cannot be implemented with other two models that used discussion threads with reference objects for geographic referencing. Although merging realtime chat with discussion threads provides a powerful and a flexible way of handling geographically referenced discussions, participants may need to train themselves with this composite function to benefit from this flexibility. 
Table 2.4 presents a summary of some research prototypes based on these models.

Table 2.4: Summary of Closely-related Research Models

\begin{tabular}{|c|c|c|c|}
\hline No & Prototype & Description & URL \\
\hline 1 & $\begin{array}{l}\text { Argumentation } \\
\text { Map (ArgooMap) }\end{array}$ & $\begin{array}{l}\text { The GIS-enabled Online Argumentation Forum } \\
\text { (ArgooMap) has been introduced by Rinner } \\
\text { (2006a). The prototype ArgooMap was designed } \\
\text { to support discussion on maps and forum threads. } \\
\text { Annotations are geographically referenced and } \\
\text { linked to geographic features. Discussion } \\
\text { contributions are structured to facilitate user } \\
\text { understanding of the evolution of ideas. }\end{array}$ & $\begin{array}{l}\text { Downloaded at: } \\
\text { http://sourceforge.net/pr } \\
\text { ojects/argoomap/ } \\
\text { Demo is not available } \\
\text { online } \\
\underline{\text { http://141.117.104.183/ }} \\
\underline{\text { argoomap/test/about.ht }} \\
\underline{\mathrm{m}}\end{array}$ \\
\hline 2 & GeoDF & $\begin{array}{l}\text { The GIS-enabled Online Discussion Forum } \\
\text { (GeoDF) has been introduced by Tang as a } \\
\text { prototype system for spatially-related discussions } \\
\text { (Tang 2006). The prototype intends to assist the } \\
\text { general public to participate in the ongoing } \\
\text { discussion of spatially-related issues. Discussion } \\
\text { contributions are structured to facilitate user } \\
\text { understanding of the evolution of ideas. They are } \\
\text { geographically referenced and linked to } \\
\text { geographic features. }\end{array}$ & $\begin{array}{l}\text { Demo is not available } \\
\text { onltp://www.vrm.ca/docu } \\
\text { ments/ACFAS09_tang. } \\
\text { based at available } \\
\text { literature on the Web. }\end{array}$ \\
\hline 3 & MapChat 1 and 2 & $\begin{array}{l}\text { The prototype MapChat supports an asynchronous } \\
\text { argumentation model with a real-time chatting } \\
\text { feature, which was introduced by Hall and Leahy } \\
\text { (2006). MapChat } 1 \text { and } 2 \text { are the Web-based } \\
\text { applications that consist of real-time protocols for } \\
\text { chatting, an asynchronous participatory approach } \\
\text { of sharing maps and imagery among }\end{array}$ & http://mapchat.ca \\
\hline
\end{tabular}




\begin{tabular}{|c|c|c|}
\hline & & $\begin{array}{l}\text { geographically distant participants and/or decision- } \\
\text { makers (originally designed and conceptualized by } \\
\text { Hall in 2003). The system was designed to support } \\
\text { discussion on maps and geo-referenced threads } \\
\text { (this feature is also known as geochatting). }\end{array}$ \\
\hline
\end{tabular}

\subsection{Review of Usability Evaluation for Web-PPGIS}

Usability evaluation of PPGIS has been comparatively less reported, especially in the context of quantitative assessment and its relationship between system usability and user characteristics (Haklay and Tobón 2003; Sidlar and Rinner 2007; Haklay and Zafiri 2008). More empirical and conceptual research is required (Jankowski and Nyerges 2001a). The methods of usability evaluation provide a sound base for the PPGIS evaluation (Haklay and Tobon 2003). Usability evaluation deals with a systematic collection of data and information related to system use for a specific task in a specific environment (Preece et al. 2002). The purpose of usability evaluation is to ensure the accessibility of computer technologies to a wide range of users (Zhao 2007). Usability deals with issues such as understanding how people use computer systems in order to develop or improve their design, and matching users' requirements so that they can carry out their tasks safely, effectively and enjoyably (Preece 1993). Usability can be evaluated in terms of efficiency, effectiveness, user satisfaction, performance speed, learnability and rate of errors (Nielsen 1993; Shneiderman 1998).

The literature provides a list of user characteristics that have a significant effect on usability. Experience, skill, education, cultural background, disabilities, software training, and user physical attributes (age and gender) are some characteristics that directly or indirectly affect usability (Thomas and Bevan 1996; Maguire et al. 1998; Jordan 1998). Moreover, Jordan (1998) suggested "a product which is usable for one person will not necessarily be usable for another". However, user friendliness and ease of system use are the main characteristics of any software (Haklay and Tobón 2003).

\subsubsection{Usability Definition, Objectives and Methodology}

Definition: Usability may be defined as the ease of use and acceptability of a prototype by the attended users who are required to carry out specific planning related workflows. Sullivan et al. (2005) also defines usability evaluation as a procedure of investigating users/participants that work together on a single platform through a Web-based 
application to determine how easily participants interact with the application interface. The components of usability testing may include:

1) the usability evaluation has particular objectives during the test of designing;

2) the participants are representative of stakeholders/general public;

3) participants work on real tasks;

4) participant's movement and computer interaction are observed and recorded; and

5) the data that is collected from the usability testing is examined to identify problems or difficulties and suggest recommendations for the improvement of the system interaction with stakeholders.

Objective: The adoption of usability testing criteria as means to evaluate the prototype proves helpful. It is widely used in object-oriented software engineering for testing computer-based software's interface design and functionalities. Moreover, this approach is useful for online GIS-based participation frameworks, which are designed using complex functionalities (e.g., GIS mapping functions) as compared to simple Internet-based applications. In short, extensive evaluation of the usability before deployment of the application is one of the reliable ways to ensure the sustained use of PPGIS by multiple stakeholders. In this research context, the objectives of a usability evaluation of web-based PGIS and/or PPGIS are to provide intended users an easy to use and interactive system whose ultimate goals are to enhance the decision-making processes through a collaborative platform, thereby increasing public participation.

Participants and Data Capture Techniques: For evaluating usability of web-based collaborative PPGIS, online participation is preferable to collect the qualitative/quantitative information. With the help of online Web-based participation, a broader range of participants belonging to different areas of education and expertise may be involved in evaluating the system usability. In order to collect data related to user performance, different techniques such as personal observation of user during testing, video and audio recording, users' comments and feedback, and a user interview and questionnaire survey are employed for usability testing (Sweeney et al. 1993).

Methods of Usability Evaluations: Inspection and user testing methods are two main types of methods for usability evaluation (Banati et al. 2006). In order to assess the usability of a software product, heuristic assessment and cognitive walk-through inspections are used. Such methods involve system experts or developers to test the product. In the user testing method, users use the product to accomplish the specific task. User testing methods specify users' performance and degree of satisfaction when users interact with the software (Butler 1996).

For usability testing (Rinner 2001; Keßler 2004; Rinner et al. 2008; Boroushaki and Malczewski 2009), every move of the user may be recorded with the help of usability tools; in turn, acquiring useful and detailed information related to the actual usage of Web-based PPGIS applications (Atterer et al. 2007). Meng and Malczewski (2009) studied the 
usability of Agroomap, in which every action, such as scrolling a page, website opening, system logging, button click, mouse move, time of events, IP address of user and filling form, in a particular order, were recorded with the help of UsaProxy software. The information that is produced during public participation is extremely important for a usability evaluation of a system. In order to collect a user profile, a user must fill out the questionnaire at the end of participation. The questionnaire is mainly comprised of demographic and socio-economic status of user such as gender, age, educational qualifications, experience related to GIS, web surfing and public participation in planning process and degree of satisfaction of using software are all important to note. The measures of usability were effectiveness, efficiency, satisfaction and learnability. To measure the effectiveness, users are expected to perform a number of tasks and express their concerns about specific issue during online participation (ISO 1998). Efficiency may be measured by calculating the time of a user to accomplish any given tasks on first attempt and by comparing time of different users. Learnability and satisfaction deal with user understanding and their positive attitude to the use of software (ISO 1998; Sidlar and Rinner 2007). The learning time of a user was quantified in order to measure learnability by noticing the time spent on the tutorial, and then, to perform a given task. Satisfaction was evaluated by asking questions related to the use of application.

\subsection{Enabling Technologies Used in Web-PPGIS}

This section focuses on how open-source software (OSS)-based technologies can be used for developing a Webbased PPGIS in order to support planning and development-related workflows. Smotritsky (2004) emphasized the details of each software product that would assist end-users and developers in the decision to choose open source technologies over proprietary solutions. He further discussed Web GIS by using Mapserver and listed hints and supportive packages for installing, troubleshooting and web link referencing to provide access to documentation of the available product. Turner (2005) evaluated the GIS software options and concluded that closed source options are limited due to restrictive licenses while an open source is better because codes are not hidden on developer sites and depict that what is going on exactly. Anderson and Moreno-Sanchez (2003) outlined five concrete issues of proprietary web-based PGIS software and concluded that OSS products for software documentation that were used in their experiment were considered more powerful, easy to learn, easily accessible and with a good level of user support that would be helpful in a decision-making process.

The requirement of a high budget during analysis, design and implementation of the GIS-based participation framework is one of the main impedances among municipalities for deploying their geospatial data over the World Wide Web. An alternative solution, which is considered as cost effective, especially for small municipalities for the implementation of GIS-based public participation processes nowadays, is the use of Open-Source Software (OSS) 
technologies. Some web-mapping application frameworks (i.e., GeoMoose, Fusion, and Mapbender similar to the Apache Software Foundation, Linux platform, the MySQL database application and many others) are successful open-source software projects. Several researchers have contributed to the study and development of OSS-based Web-PPGIS to support public participation and enhance decision making-related processes during municipal planning. PPGIS prototypes such as MapChat and AgrooMap have been developed by Hall and Leahy (2006) and Rinner (1999, 2001) using OSS-based technologies to support asynchronous participation approaches. Similarly, Butt and $\mathrm{Li}$ (2012) developed a prototype of an integrated online GIS-enabled virtual public meeting space (GeoVPMS) system based on OSS technologies. The GeoVPMS framework integrated the open map services and open source based solutions to enable Web-based tools to support public participation in decision-making and municipal planning processes. The prototypes designed and developed based on OSS technologies and free OS-GIS provide cost effective solutions for small municipalities. The advantages of the OSS technologies are discussed in next section.

Open-Source Software (OSS) refers to software that has been made available under a free software license with the rights to run the program for any purpose (Bill and Korduanp 2004). Open-source software has a special type of license, such as the General Public License (GPL), ensuring free distribution, change and access to the source codes. Tsou and Smith (2011) state free software is different from open-source software and is usually distinguished through the license it has. Software like MapServer, does not incur any costs for updates and maintenance, whereas the proprietary software ArcServer can cost as much as (under ArcGIS Server Workgroup standard license fees ${ }^{2}$ ):

- Initial License Fee and First Year's Maintenance: \$5,500 approx. (Available in 30-, 90-, and 365-day terms and range from $\$ 600$ to $\$ 2000$ based on term length and cores per server).

- Annual Maintenance Fee from Year 2 onwards: range from $\$ 2000$ to $\$ 3300$ approx.

- Initial Training Costs (desktop GIS only) range starts from $\$ 500$ approx.

${ }^{2}$ Information related to the license fees is obtained from the following websites (last accessed in Jan 2015): http://www.ogs.state.ny.us/purchase/prices/7600020751prices.pdf or http://des.wa.gov/SiteCollectionDocuments/ContractingPurchasing/esri/ESRI PriceList Sept2014.pdf 
Figure 2.14, developed based on Tsou and Smith (2011), illustrates the categories of free and non-free software.

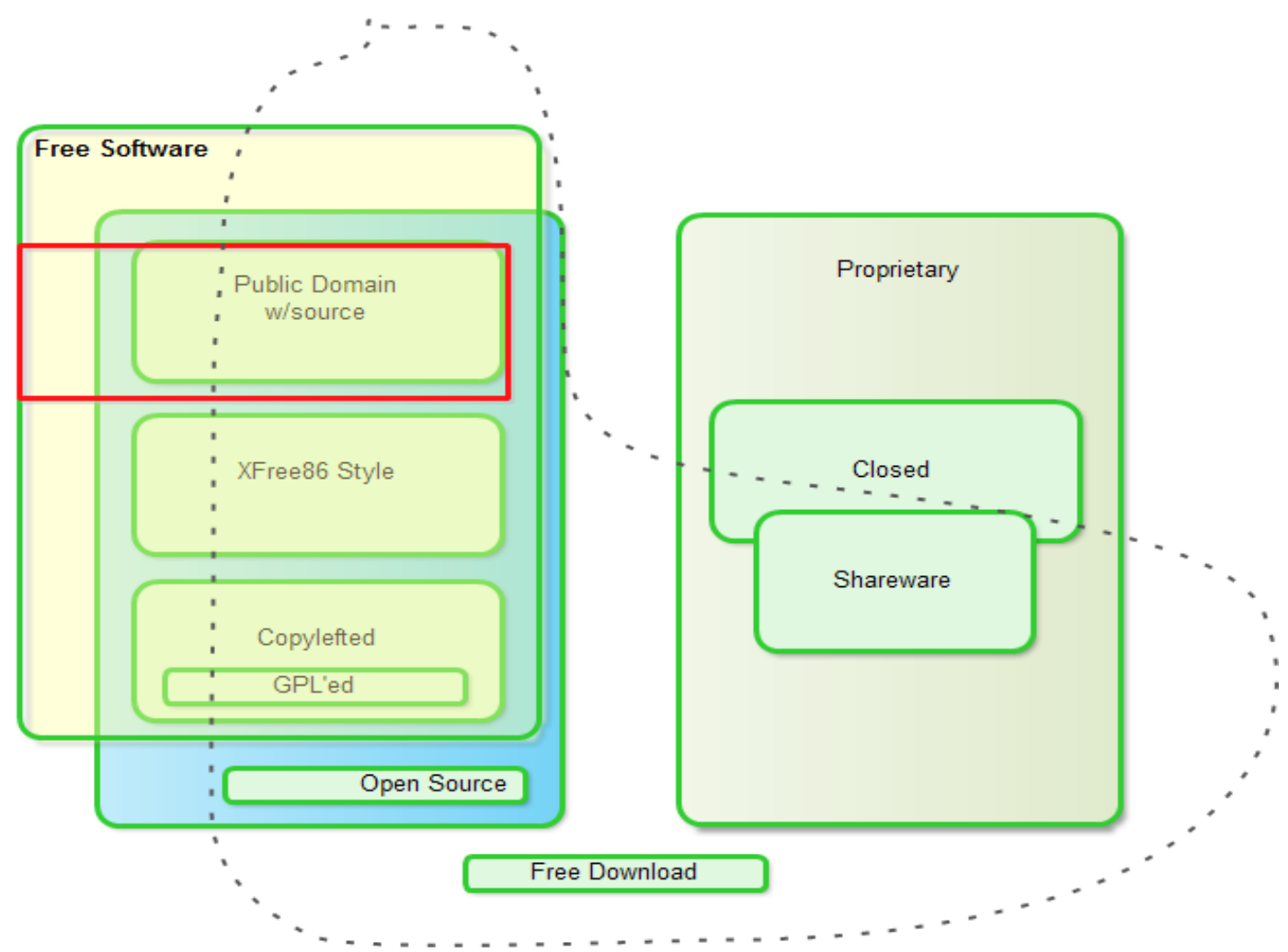

Figure 2.14: Modified Categories of Free and Non-Free Software (http://www.gnu.org/philosophy/categories.html)

In Figure 2.14, the red box highlights public domain software that is open source, some of which are freely downloadable. Some proprietary software may be freely downloadable too (e.g., Google Earth). Copylefted is the term given when a copyrighted work is given freedom to be used and distributed freely. Many non-profit forums work actively to organize and encourage the use of open-source software. The development process for open-source software includes peer reviews, standard control and an active community ensuring a high quality and reliability of the resulting end product. Community helps provides a resource area for the open-source software available, as well as maintains standards, and provides licenses, legal coverage, funding and easy access. Open Geospatial Consortium (OGC) is an international consortium, consisting of more than 420 companies, government agencies, research organizations, and universities. OGC plays a role in a consensus process to develop a publicly available geospatial interface for exchanging and processing geospatial information through defining format, standards and services. 
It is responsible for defining open standards, which are defined in Figure 2.15.

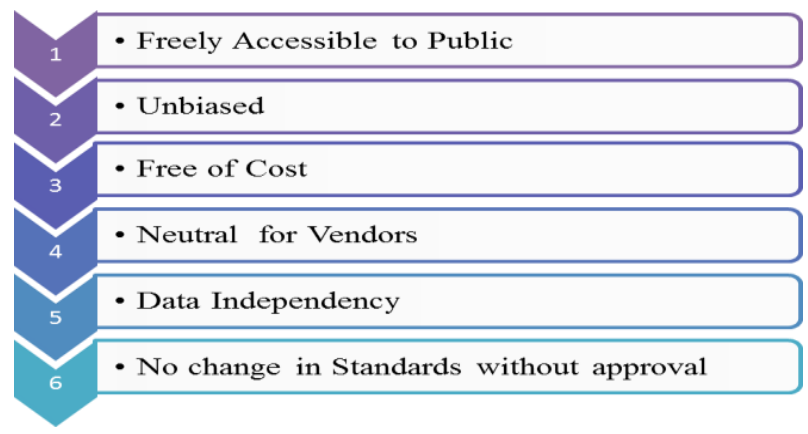

Figure 2.15: List of Criteria Showing How OGC Standards are Developed

Software developers use these criteria to build interfaces and encoding (into their products and services) that make sharing of information possible among geo-processing systems from different developers, whether employed by proprietary product vendors, independent integrators, application developers or active in open source projects. Similarly, the Open-Source Initiative (OSI) is a "non-profit corporation with global scope formed to educate about and advocate for the benefits of open source and to build bridges between different constituencies in the open-source community" (OSI 2007).

\subsubsection{Advantages of OSS-based Technologies Towards PPGIS}

In the software development process, the use of OSS technologies helps a lot to obtain several benefits like low cost, reliability, freedom, auditability, support of active users, and security/stability. Nowadays, a large number of organizations recognize the ultimate benefits of merging OSS as an essential part of their business development plan. As in recent times, most of the GIS communities and GIT industries are considering the incorporation of OSS technologies during the development of GIS-based programs, methods, processes, tools and techniques (Mapserver 2004). Based on OSS technologies, PPGIS application development is cost effective, secure, easily accessible, and easy to learn (Lakhani and Von 2003). The requirement of a high budget during the analysis, design and implementation of the GIS-based participation framework is one of the main impedances for small municipalities to deploy their geospatial data over the World Wide Web (Li et al. 2006). The advantages of OSS technologies, which may be helpful and can be used by the developer in the development of PPGIS-based collaborative applications, are as follows:

- Non-discriminatory: OSS are available to anyone, any organization, anytime, anywhere with no restrictions. Anyone can download the source code and modify it for its betterment. 
- Cost Effective: OSS is publicly available without any charges and unencumbered by patents and other intellectual property. The primary goals of OSS technologies are to provide cost effective solutions for low budget organizations as most OSS projects are available free of charge and royalty.

- Reliability: Broadly speaking, in the computer science field, we can measure software reliability with the absence of defects, such as data loss or sudden failure. Sometimes, the OSS is more reliable if it has fewer bugs or mistakes, which are usually eliminated with the involvement of different work groups due to their collaborative efforts, feedback and discussions.

- Auditability: The source code of an OSS-based application can be audited or inspected by any interested individual/firm to make it compatible. Therefore, all concerned departments can also be auditors with full liberty (Grassmuck and Volker 2004).

- Support of Active Users' Mailing List: All the active users developing any framework based on OSS technology can identify problems with the help of one another and expect that other members will fix them and deliver their solution easily and freely. Recently, the open source community has become very powerful in the GIS domain.

- Availability: OSS is easily available as some of the software developers' communities are also working as part of the OSS communities to get the benefit of having tools available from the communities to promote public participation.

- Security/Stability: As there is a big community of OSS users/developers, OSS application has been demonstrated to be secure because of fast tailoring and frequent examination of source code by different developers.

- Vendor/Data Neutral: OSS is a vendor neutral in terms of their content and implementation concept and does not favor any vendor over another. The standards are independent of any data-storage model or format. Free open source, without limitations to its distribution and access for public use and maintenance, helps make an open source a good candidate for public participatory GIS applications.

- Documented: Mostly, OSS is defined, documented and approved by a formal, member-driven consensus process. The consensus group remains responsible of changes, and no single entity controls the standards. The OGC's open standards are specifications for interfaces and encodings that enable interoperability between geo-processing systems from different developers, whether employed by proprietary product vendors, independent integrators, application developers or active in open source projects.

In brief, the above-mentioned benefits are a broad-spectrum and can be applied to any software development process, including IT, GIT, and GIS. 


\subsection{Discussion}

Multi-collaboration is important in participatory planning practices. A number of researchers have paid attention to Web-based PPGIS frameworks that support collaborative experiences among geographically dispersed participants during participation processes. The design and implementation of the asynchronous participation approach-based research prototypes - e.g., MapChat, ArgooMap, Argumentation Map and GeoDF by Hall and Leahy (2008), Rinner et al. (2008), Keßler (2004) and Tang (2006) - support the decision-making process, increase stakeholders participation to provide frequent feedback, and broaden the use of map data by the participants. However, these prototypes have functional similarities in relation to the model developed by Rinner (1999). For instance, the argumentation model supports a geo-referenced based forum mapping technique, which means that each element of discussion and/or argument has been linked with a geographic reference object on a map. Similarly, some other researchers such as Chang (2010), Jiansheng (2007), Zhao (2007), Ma (2006), Gu (2004) and Li (2002) have worked to encourage participation approaches using a collaborative synchronous framework, Web-based collaborative GIS and open source software solutions. A synchronous participatory approach was developed to support collaboration among users (Chang 2010). However, it has not been used in a Web-PPGIS and/or municipal planning context, such as real-time spatial data sharing among public participants and/or policy makers during public meetings related to municipal planning workflows. In brief, little has been done on designing and developing such online map-sharing tools to support real-time synchronous collaboration.

The development of synchronous collaborative PPGIS applications will be one of the positive steps taken by researchers for the solution of municipal planning related problems (Butt and Li 2010). Research related to the design and implementation of synchronous collaborative PPGIS is still in an early stage of development. Thus, there is not much literature in this field. As a result, there have only been a few and/or inadequate empirical studies

addressing the synchronous collaborative Web-PPGIS and groupware-based spatial decision-making designed to facilitate collaborative work (MacEachren 2000; Boroushaki and Malczewski 2010). The rapid changes in technology, especially, in the field of GIS, OSGIS, GIT, CSCW, groupware, Internet computing technology, virtual reality, geographic visualization, geo-collaborative technology and others, will have a significant influence on the need to innovate a collaborative synchronous Web-PPGIS.

By examining the researchers' contributions from the literature review, this research seeks to outline the significance of ensuring the implementation of valuable and adequate methods, techniques and tools (i.e., map/data sharing mechanism discussed in next chapter) to improve the municipal planning process workflows. The objective is to ensure that the proposed research infrastructure, with the synchronous and asynchronous approaches to support 
sharing of information, will help to improve/increase public participation and/or lead decision-makers to a final and effective decision in a timely fashion.

\section{Summary}

The Web-PPGIS initiates a new idea by using online geo-referenced participation forums, which are rapidly becoming a useful means for the stakeholders in the planning and development processes. It also helps to explore spatial context, at least in those places or environments where public participation is required (Simão et al. 2009; Li et al. 2007; Li and Ma 2006; Rinner 2006; Tang and Coleman 2005; Hackney's GIS Team 2004; Orlando GIS 2004; Meredith 2000; Evans et al. 1999; Jankowski et al. 1997). High-performance computing technologies, ICT, groupware and collaborative GIS are facilitating the advancement/innovation of PPGIS for improving GIS-enabled public participation within the planning and development context (Faber et al. 1998; Ghose 2001; Jankowski and Nyerges 2001a; Craig et al. 2002; Allen 2003; Balram et al. 2003; Ban 2003; Balram and Dragicevic 2006a; Jankowski 2009). Several researchers have made tremendous contributions to the study of PPGIS. Up to this point, most of the PPGIS applications, such as AgrooMap, MapChat and GeoDF have been focusing on supporting asynchronous participation approaches (i.e., public participation using geo-referenced forums).

This research considers public participation as an input during municipal planning processes for effective and efficient decision-making. It advocates the idea that collaborative PPGIS, real-time CSCW and groupware technologies offer an excellent means for bringing/gathering the stakeholders, public and organizations anytime anywhere during the planning process to promote collaborative decision-making. The main goal is the use of Webbased collaborative PPGIS as a tool for sharing ideas/knowledge among different interested groups or stakeholders such as planners, decision makers or higher authorities, and most importantly, the public.

The next chapter describes the architecture and design of the real-time Co-PPGIS infrastructure to show its efficiency and reliability in different situations related to municipal planning. 


\section{Chapter 3 Design Requirements for Modeling Co-PPGIS}

The success of designing and deploying a web-based Co-PPGIS, in order to enhance the degree of public participation to support collaborative decision-making during municipal planning and management workflows, mainly depends on comprehensive understanding of: (1) the concepts of public participation in planning and management - including basic concepts of participation, degree of public participation, and existing participation practices during municipal planning, development and management; and (2) functional and non-functional requirements, identified from existing PPGIS, and closely related research models, which have been developed to support the public participatory processes during municipal planning and management (discussed in Chapter 2).

This chapter begins with an overview and explanation of a Co-PPGIS concept, which describes Co-PPGIS perspectives. The second section describes the Co-PPGIS conceptual framework, which implements the concepts of real-time synchronous and asynchronous participatory approaches to facilitate the decision-makers to make decisions in time by incorporating public input during the municipal planning process. It provides an introductory source of information to: 1) understand a Co-PPGIS meeting infrastructure, 2) identify the gaps in existing public participation practices and possible improvements that can be made by adopting the suggested Co-PPGIS, 3) identify direct relationships between the Arnstein (1969) and Weidemann and Femers (1993) ladders of participation and Co-PPGIS concept, and 4) deal with the requirements modeling for Co-PPGIS (i.e., methodology adopted for requirements engineering of Co-PPGIS followed by a discussion on its high-level architecture and data flow models).

\subsection{A Concept of Co-PPGIS Perspectives}

In many developed countries, municipal and local government by-laws require certain level of public participation in their planning and decision-making processes. Besides, the management of geospatial data, especially during the planning, development and management of municipality, city and/or state, remains a challenge (Li et al. 2007). CoPPGIS is a real-time geospatial Web conferencing infrastructure, which provides planning and management related spatial/non-spatial information to higher authorities, decision makers, and government bodies on a real-time basis. In this research, the proposed Co-PPGIS is focusing on municipal projects through promoting a GIS-enabled virtual meeting concept. 
The Co-PPGIS concept is described from five perspectives (see Figure 3.1), which are briefly discussed below:

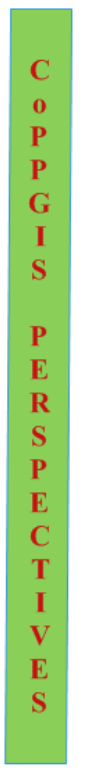

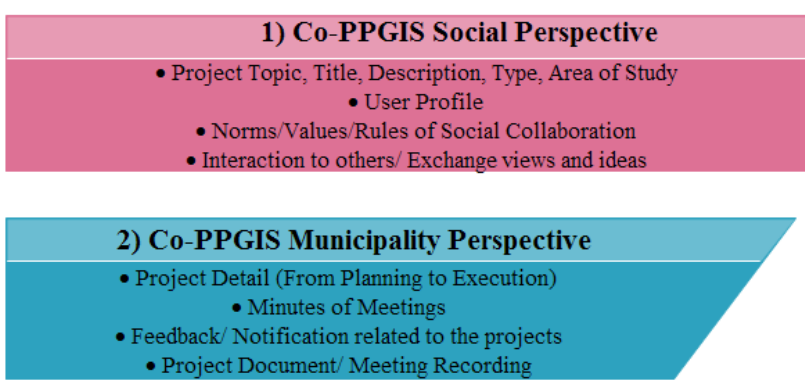

4) Co-PPGIS Geo-Spatial Perspective

- Spatial Data (Area of Interest, Geographic Data)

- Municipal project spatial context better understanding

- Synchronous and Asynchronous Spatial/Map data Sharing

- Tools (Zoom in Zoom out, draw, Navigation on Map, Highlight parcel)

5) Co-PPGIS Virtual Meeting Environment Perspective - Location free

- Live Feedback/Better Collaborative Decision Making

- Real-time Public Participation (Sync and Async Participation approaches)

- Complementing to the Participation and GeoSpatial Perspectives
3) Co-PPGIS Participation Perspective

Concept refers to Synchronous
and asynchronous public participatory support

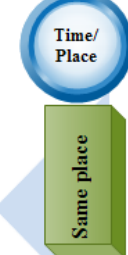

\begin{tabular}{|l|}
\hline \multicolumn{1}{|c|}{$\begin{array}{c}\text { Same time } \\
\text { (Sync Participation) }\end{array}$} \\
\hline $\begin{array}{l}\text { Face-to-face (public } \\
\text { meeting, public } \\
\text { conferences) and } \\
\text { virtual meeting } \\
\text { environment }\end{array}$ \\
\hline
\end{tabular}

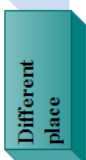

Synchronous CSCW

environment

(groupware, video-

and audio

conferencing tools)

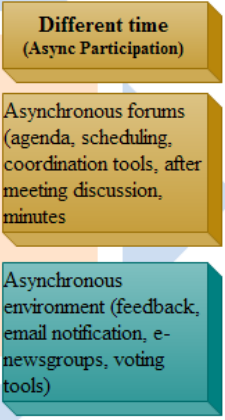

Co-PPGIS Participation

Perspective w.r.t. Time Place

$$
\text { Matrix }
$$

Modified after (Johansen 1998, Dix 1996)

Figure 3.1: Co-PPGIS Perspectives (Butt and Li 2014)

Co-PPGIS Social / Socio-spatial Perspective: The social/socio-spatial perspective in the Co-PPGIS is to show and highlight a project topic (i.e., name/title, small description, type of project, area of study), which helps stakeholders to participate in the relevant project or matter. Submission of a user profile before joining the meeting assists stakeholders to identify the status of all participants. There are some norms, values and rules for social interaction between participants and the existing infrastructure. As the participants join the meeting session, their interaction level rises. They exchange their views and ideas, which lead to better decision-making for municipal projects.

Co-PPGIS Municipality Perspective: In any municipal related project, the concept of all information about a project at one place is very important. This helps to differentiate the status of one project from another project in the same domain. The proposed Co-PPGIS municipality perspective has the facility to collect and maintain data (e.g., planning information, drawings/maps, minutes of previous meetings, meeting recordings, feedback form, notification, project documents) at one place where the participants can easily get the information at the level that they want.

Co-PPGIS Participation Perspective: The Co-PPGIS participation perspective refers to synchronous and asynchronous public participatory approaches during municipal planning related workflows. It encourages 
participants to be involved in a municipal project based on their own convenience. A group of participants can share their views using maps, drawing tools, rich multimedia, and project documents through a synchronous approach, in which multiple participants can see what the other person is doing at the same time without wasting a second. A video chat is the best example of a synchronous approach, in which everyone can see and understand what the other is performing. Supporting indirect communication among stakeholders is also available through an asynchronous approach, in which it is not necessary to see what the other is saying at the same time. Filling a feedback form and sending an email message are examples of indirect communication among stakeholders. The Co-PPGIS participation perspective provides both direct and indirect communication facilities for ensuring participation of stakeholders according to their convenience. The Co-PPGIS participation perspective also defines the ways in which participants collaborate with each using Co-PPGIS Asynchronous Participatory Environment (CAPE) and CoPPGIS Synchronous Participatory Environment (CSPE) (e.g., using GIS-based discussion forums and GIS-enabled virtual meeting platform). The same Co-PPGIS concept can be described according to the temporal and spatial dimensions used in a time-place matrix of Johansen (1988) and Dix (1998) as: 1) same time and same place (CSPE); 2) different time and same place (CAPE); 3) different time and different place (CAPE); and 4) same time and different place (CSPE).

Co-PPGIS Geo-Spatial Perspective: This concept relates to synchronous and asynchronous map-sharing between participants for a better understanding of the spatial context of a municipal project. The proposed prototype uses GIS technology through which a participant can envision an area of interest, navigate on the map, draw or highlight any patch on the map. Furthermore, the participants can invite others for real-time synchronous collaborative discussions with a shared map view on any area of interest. Moreover, they can discuss municipal planning and development related issues and propose solutions collaboratively for better decision making.

Co-PPGIS Virtual Meeting Environment Perspective: This concept is a combination and/or complement to the participation and geospatial perspectives. In this tiring life, it is not always possible for a stakeholder to come and attend a meeting at a fixed place. The time has changed, and technology has become more advanced and userfriendly. The proposed Co-PPGIS provides the option of a virtual meeting in which the physical attendance of a participant is not necessary. Participants can easily participate through this electronic meeting facility and share his/her views related to municipal projects. Participants can use video chatting with mapping flair to express their point of view. Participants can drop a message for a specific participant from anywhere (on a map) without the restriction of place and time. In this way, stakeholders can easily become involved in any project of their interest that is being developed for a municipality. There is an immense need to develop such collaborative infrastructures in developing countries like Pakistan, and India where resources are minimal. In brief, a Co-PPGIS virtual meeting 55 
environment is an online meeting mechanism used to support group collaborations on textual information, rich multimedia, project documents as well as geographical information, such as maps and imageries, and capturing and sharing of local/domain knowledge, in real-time during municipal planning and management.

Figure 3.2 shows Co-PPGIS virtual meeting workflow processes, service abilities, and situations when its functional capabilities are useful.

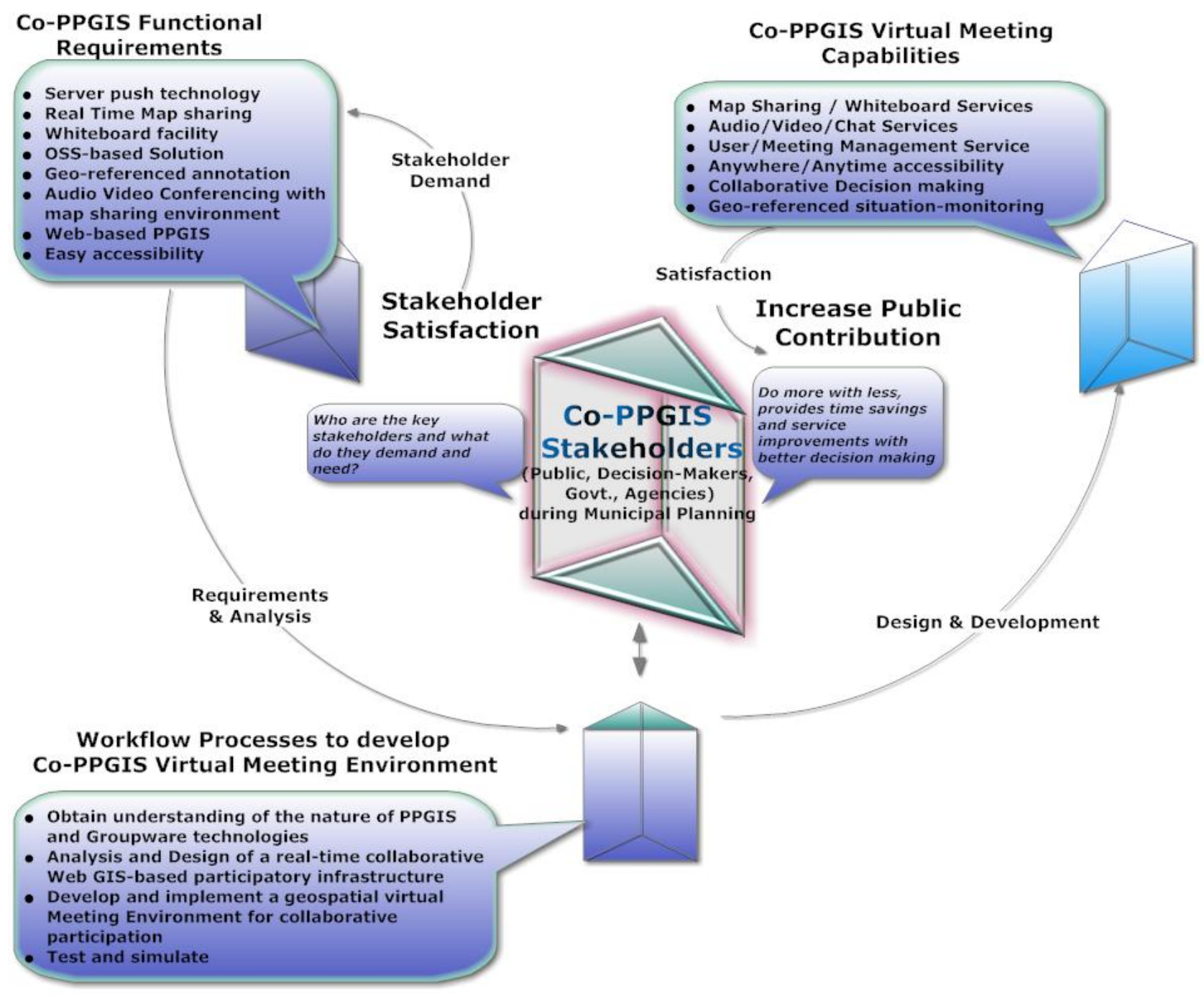

Figure 3.2: Co-PPGIS Workflow Processes and Service Abilities (Butt and Li 2014) 
The virtual meeting environment allows for the integration of geospatial data from different sources in the form of web services, and collaborators' input through geo-referenced annotations. It includes integrated components such as map-sharing, audio/video conferencing, real-time chatting, geo-referenced textual and graphical annotation and user/session management (the details of these components are explained in Chapter 4).

\subsection{Co-PPGIS Conceptual Framework}

A concept is a plan, intention or a mental image of a thing, entity or a class, whereas a framework is defined as a structure which provides support to a number of components and serves as a wrapper (Butt and Li 2014). In other words, a conceptual framework is a plan or structure of interlinked concepts, which provides support to build understanding of a certain phenomenon or process.

Co-PPGIS can be defined conceptually as the uses and applications of Geographic Information (GI) and/or GIS technology used by the public, both as individuals (asynchronous) and grass-root groups (groupware synchronous), for participation in the municipal processes (data collection, mapping, analysis and/or decision-making) (Butt and $\mathrm{Li}$ 2014). It particularly focuses on municipal planning and management paradigm, such as the provision of everything to large scale community (i.e., general public), to gain public participation and to acquire the involvement of small scale stakeholders (i.e., decision-makers and higher authorities) in the collaborative decision-making process. CoPPGIS works as a tool and opportunity to explore geographic locations of opinions and to compare technical evaluations across the determined or non-determined spatial extent (De Meo et al. 2011).

Section 3.1 illustrates five different perspectives of Co-PPGIS, one of which is the virtual meeting environment perspective, which allows for more synchronous public participation in the process of analysis, sharing thoughts, and collaborative decision making. The main emphasis of the Co-PPGIS concept is on who is participating (individual or group interaction), in what they participate (mapping, analysis, and decision-making) and how they participate (means of participation i.e., annotation, arguments, comments, audio/video streaming, rich multimedia and document sharing, etc.). The aim of this attempt is to formalize the conceptual framework of Co-PPGIS developed for incorporating effective public participation through collaborative discussion and decisions in planning related problems. This Co-PPGIS conceptual framework is basically a blend of asynchronous and synchronous participation approaches to collaborate effectively for any spatial planning phenomenon/problem. Co-PPGIS uses GI-Technologies along with Geo-information to facilitate people (i.e., public, developers, decision authorities and researchers) in group decision making processes. 
Figure 3.3 depicts the core concept of Co-PPGIS.

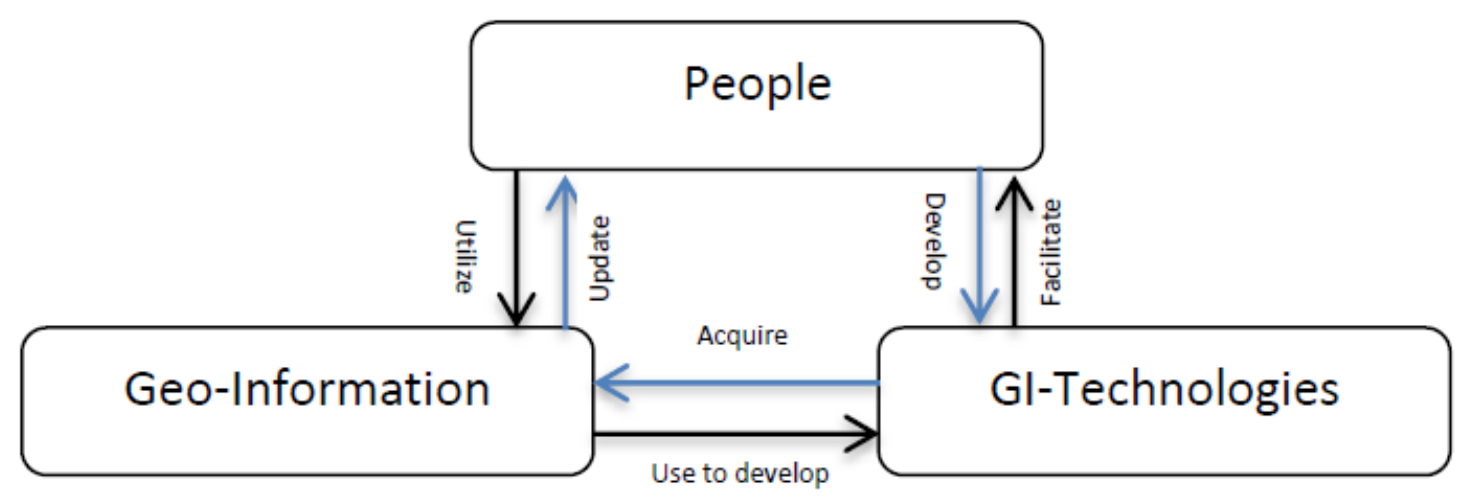

Figure 3.3: Co-PPGIS Core Concept of Interaction

The main concept of synchronous and asynchronous participation approaches to Co-PPGIS is to reduce socio-spatial and technical and technological barriers throughout the globe using a time-place matrix (e.g., a group of individuals can interact with each other at same time anywhere irrespective of any other (social, technical and knowledge) factor). Co-PPGIS's virtual meeting environment uses real-time map-sharing as a sort of synchronize room-based mechanism in which a virtual room is considered a predominant metaphor for organizing flexible interaction amongst participants; and thus, forms an interactive virtual online collaborative map-sharing environment. It is an innovative and interactive approach to facilitate public participation and collaborative decision-making that is different from traditional thread-based PPGIS collaborative mechanisms For instance, asynchronous PPGIS are not real-time in sharing the maps and discussions among public/stakeholders. Any municipal project, decision or results of a planning activity can be discussed by the public at different spatial location, with different mentality, geographical and technical knowledge.

The main purpose to design this Co-PPGIS framework is to enhance the current PPGIS framework and to facilitate public involvement in the existing practice of municipal planning in order to grasp multi-dimensional events, comments and reviews that can be viewed collaboratively on a map somewhere globally via the WWW (i.e., converting visual thinking (private realm/domain of any individual) to visual communication in real-time).

Principally it is an effort to give voice to unspoken. Digital objects or information sources in this Co-PPGIS are technical planning maps, planning documents, guidelines, picture, audio, video, and discussions/comments/reviews as electronic messages, conferencing and online map-sharing. Any types of supporting documents, maps, rich multimedia are accessible and exchangeable among participants during the group discussion. 
Figure 3.4 shows the goals of Co-PPGIS with respect to its core elements of interactions (i.e., spatial, social, and technology).

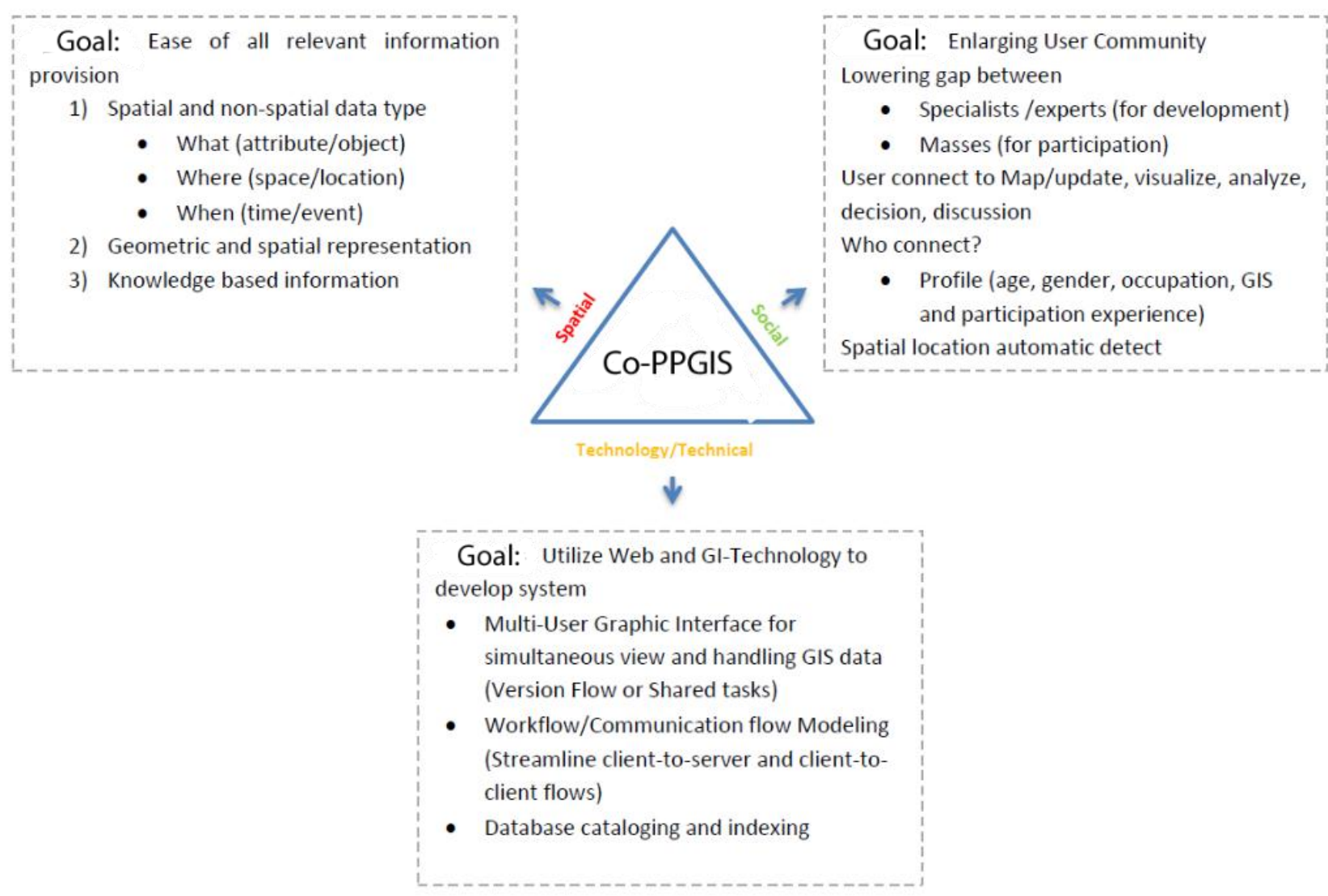

Figure 3.4: Co-PPGIS Goals with respect to Core Elements of Interactions (Butt and Li 2014)

The basic advantage of this Co-PPGIS framework is to have immediate and/or gradual feedback by a whole group of individuals involved in planning related activities. The Co-PPGIS facilitates participants to navigate on the map (synchronously and asynchronously map-sharing), explore (the functionality and purpose), analyze (the system and phenomenon under consideration), and finally participate through annotating, viewing, knowledge sharing, and commenting. The Co-PPGIS archive for finished discussions could be made available for further considerations.

Figure 3.5 represents the stages of the theoretical framework of Co-PPGIS. Theoretically, the framework of the CoPPGIS includes identification as well as defining spatial and non-spatial data structure, objects and sources needed 
under a virtual meeting environment along with their required physical/social characteristics. The role of the user is pre-defined by administrator, with the interaction of users among themselves and the objects, and finalized under the workflow model. For finalizing elements of collaboration, various incorporating elements of the system are analyzed and decided for implementation. For example, the Co-PPGIS system includes composing, connecting and influencing elements (see Figure 3.5).

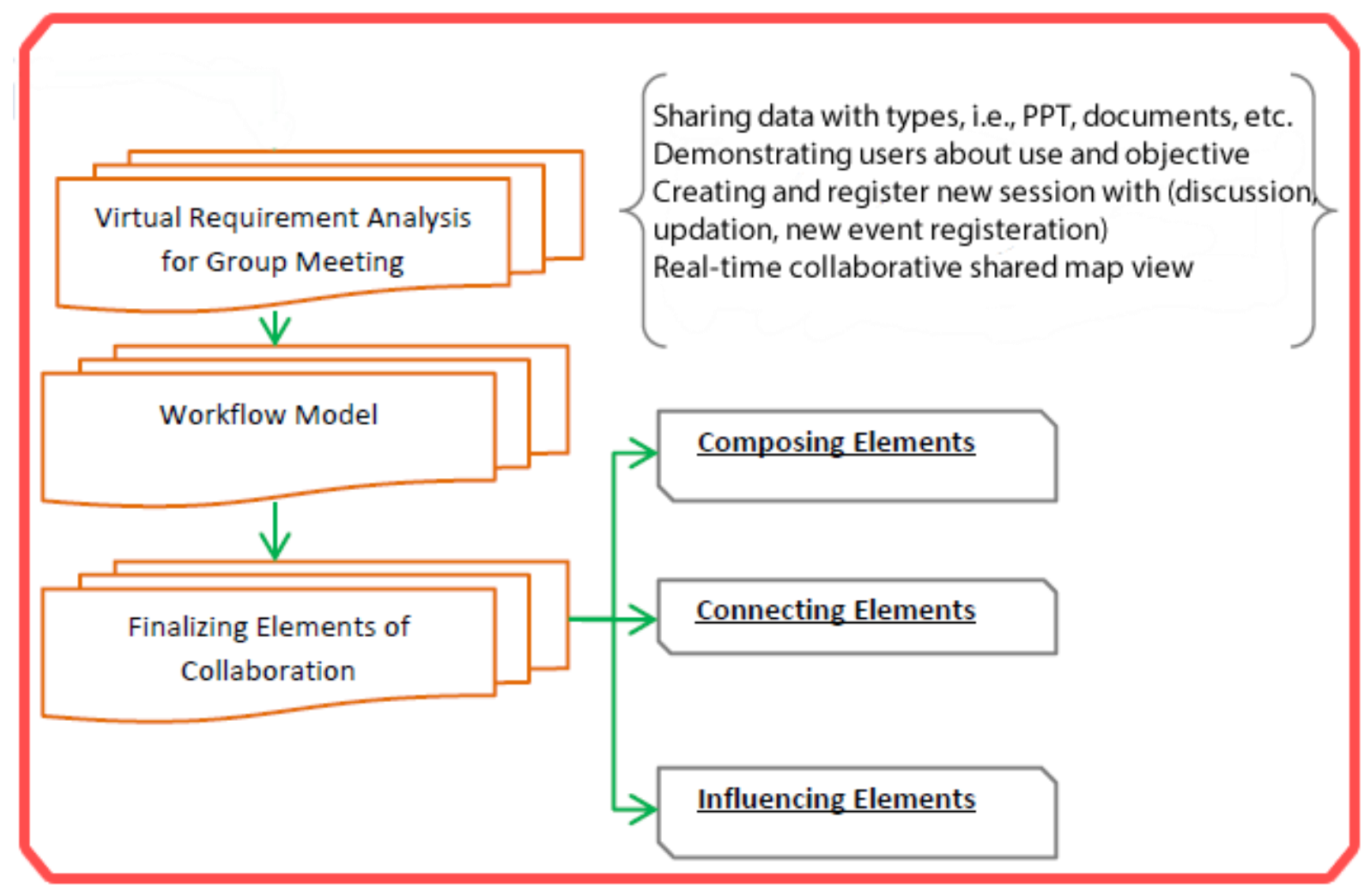

Figure 3.5: Stages of Theoretical Framework of Co-PPGIS

Composing elements are elements forming the Co-PPGIS system in its form that include data, software, and mechanism. Connecting elements are the methods of communication in Co-PPGIS that include access and participation ways (i.e., map-sharing, chatting, rich multimedia sharing, document sharing, audio/video conferencing, draw graphics/annotation, and display charts). Influencing elements are those that may or may not influence the system and include cultural, social, technological or technical aspects. 


\subsection{Understanding a Co-PPGIS Meeting Infrastructure}

The proposed Co-PPGIS is a multifunctional GIS-enabled, collaborative virtual meeting participatory infrastructure, which integrates different information technology tools to support participation and collaboration activities before, during and after public meetings. It centers on public meetings held during municipal planning-related activities. Before public meetings, the major activities focus on information access, communication and collaboration of stakeholders. During the meetings, real-time access to the meetings and their presentations become major tasks. After the meetings, the focus shifts to the presentation of syntheses of public participation, access to decisions, and receiving of feedbacks. The proposed Co-PPGIS is a combination of a synchronous and asynchronous mechanism of participation, interaction, communication, and mainly spatial data sharing among participants that has been implemented in several sub-components discussed later in the prototype implementation chapter.

Figure 3.6 shows different level of interaction of stakeholders (i.e., before, during, and after public meeting) with the Co-PPGIS virtual meeting infrastructure.

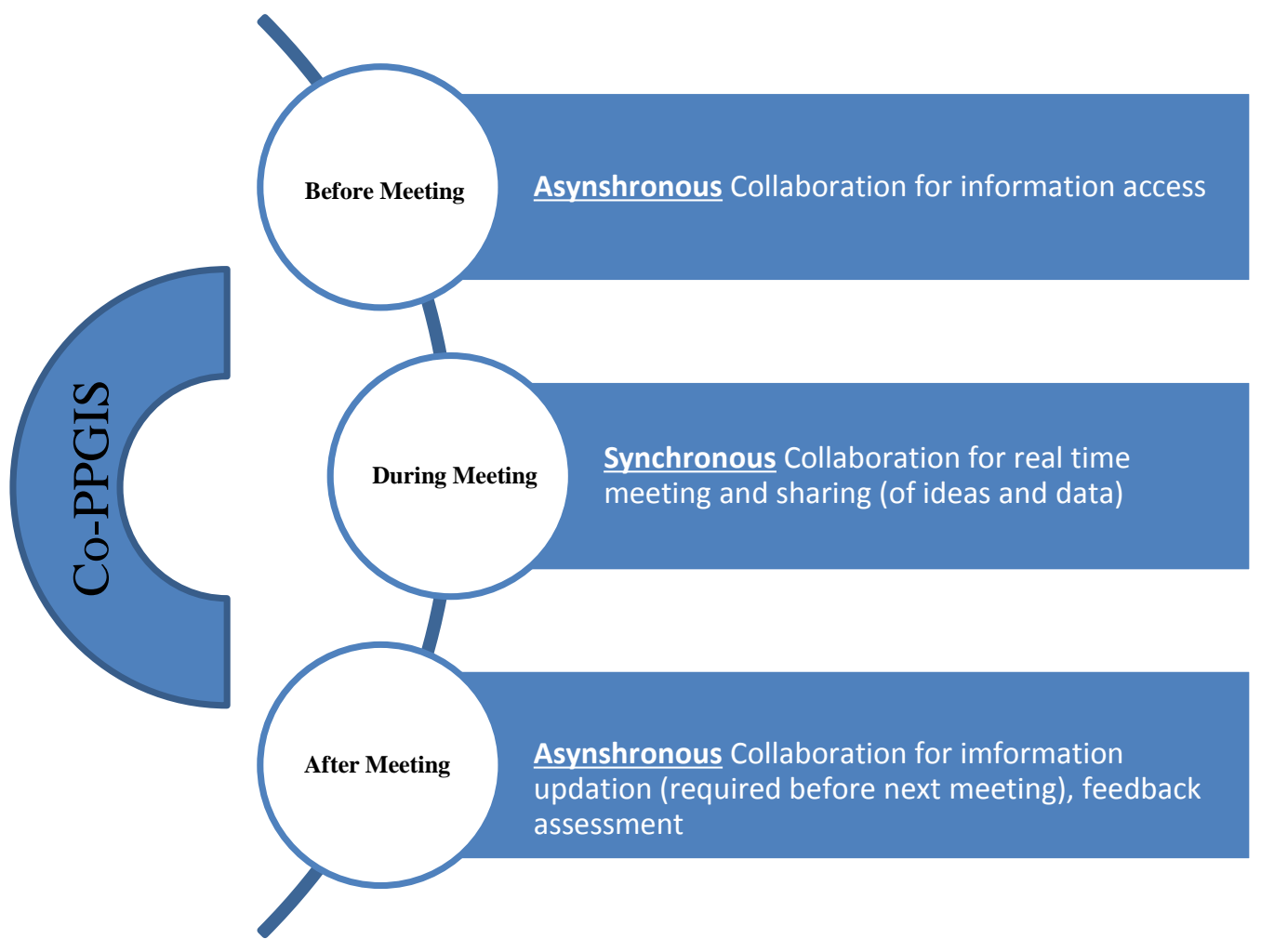

Figure 3.6: Public Meeting Scenario at Three Stages/ levels of Interaction 
In order to understand a desired system completely, Co-PPGIS may be categorized as and/or mainly composed of two major application infrastructures.

Figure 3.7 shows two major Co-PPGIS synchronous and asynchronous concepts for the municipal planning and management related activities which are describes as follows:

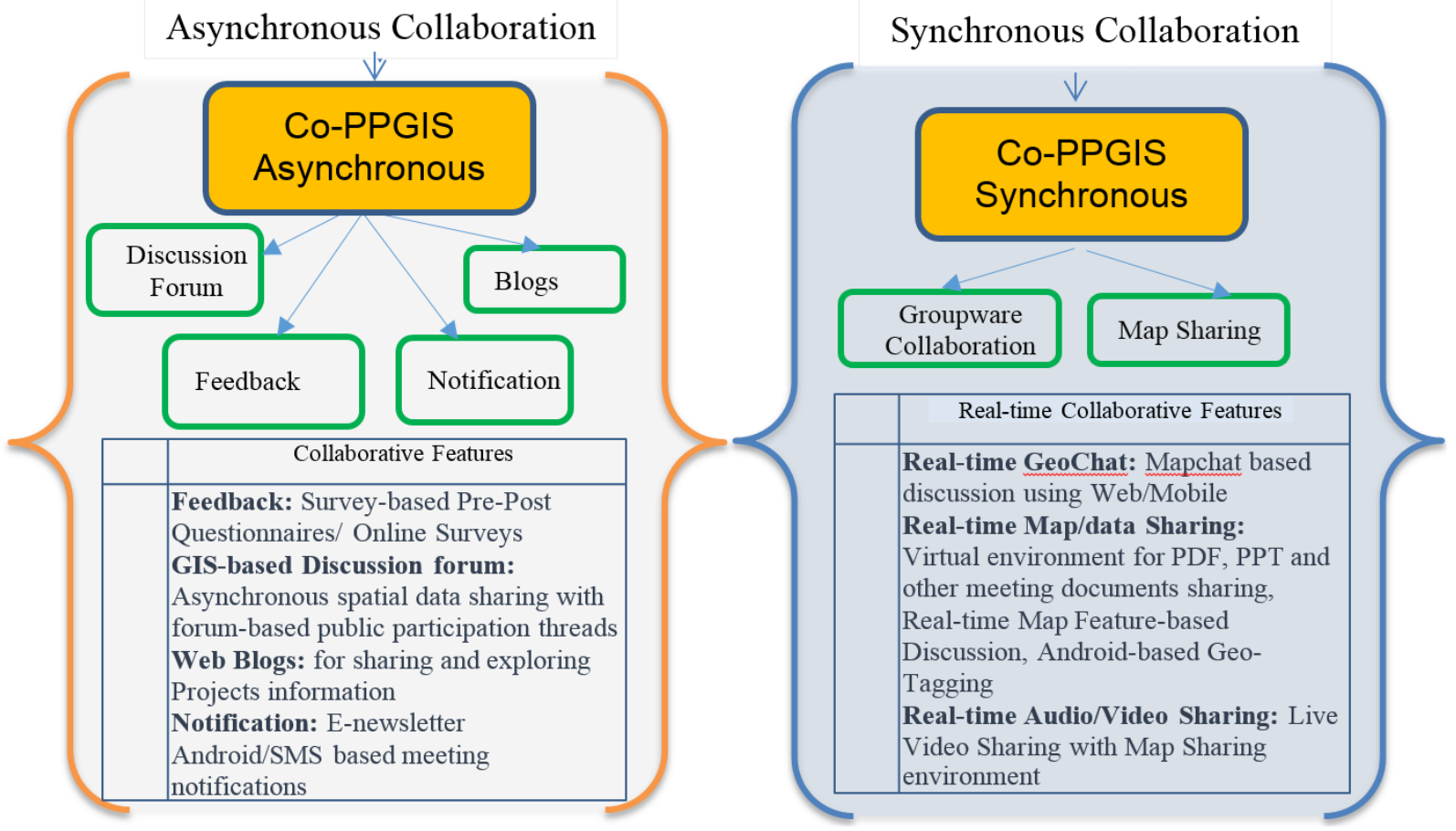

Figure 3.7: Co-PPGIS Synchronous and Asynchronous Concept for Planning and Management (Butt and Li 2012)

1) Co-PPGIS synchronous participatory map-sharing environment: the synchronous collaborative GIS processes are implemented by applying CSCW or groupware application principles. In this way, participants can share comments, ideas or suggestions after exploring spatial data by using digital multimedia tools and technologies.

In brief, decision-making authorities can easily manage multiple projects by assigning room permission levels (i.e., public, private and scheduled meeting rooms) on a project-by-project basis. They can also use real-time map-based sessions for ideas and information exchange, clarification of concepts and gaining essential public input before making any final decision related to municipal planning and development. 
2) Co-PPGIS asynchronous participatory environment: Web-based GIS geo-referenced discussion forum and/or GIS Blog techniques are used to support public input and discussion.

Another logical perspective of Co-PPGIS in connection with municipal planning workflows is shown in Figure 3.8. Each module of Co-PPGIS has its own functionality that contributes in raising public participation at each level of the planning and development process. The main functionalities include: 1) discussing and annotating maps anytime, anywhere by everyone whether participants are in the office, at home or on the road; 2) access to relevant planning and development documents from any browser; 3) effective and efficient decision-making process; 4) project planning efficiency and collaboration with others to consolidate, manage and communicate project plans, share necessary documents, and update project status; and 5) enhancing participants' options for communication (e.g., real-time map-sharing and project documents sharing) through which the attendees can collaborate and share their ideas in real-time.

As shown in Figure 3.8, the Co-PPGIS asynchronous environment includes a municipal project information blog module, which manages and provides access to information related to an ongoing municipal planning and development project (i.e., notifications of public meetings, meeting outputs, public input, and project related documents) to the public and other stakeholders. 




Figure 3.8: Logical Perspective in Relation to Municipal Planning using Co-PPGIS (Butt and Li 2012)

Furthermore, the Co-PPGIS asynchronous module allows participants to explore map data using the spatial data handler component and to discuss issues related to municipal planning through a GIS-based forum. The Co-PPGIS synchronous module, together with open map API's and real-time collaborative map-sharing modules, provide realtime access to the public meeting to those who are not able to attend physically. This research focuses not only on developing a real-time map (or geospatial information) sharing mechanism, but also integration of open sourcebased groupware solutions in a Co-PPGIS synchronous participatory environment to facilitate participants with CSCW functionalities, such as screen sharing, video conferencing, and document sharing. 


\subsection{Gaps in Existing Municipal Planning Practices and Possible Improvements using Co- PPGIS}

This section describes a brief understanding of the proposed Co-PPGIS and the possible improvements using CoPPGIS by identifying gaps in the existing municipal planning process workflows. These gaps are attained by: 1) literature review; 2) observing existing public participation practices; 3) reviewing/comparing functionality of existing PPGIS applications and at the end identifying the functionality gaps between those collaborative PPGIS applications, which formed the essential basis for Co-PPGIS requirement analysis and architectural design (briefly discussed in Section 3.7 and 3.8).

Figure 3.9 portrays the existing participation practices, methods and proposed research infrastructure of asynchronous and synchronous collaborative participatory approaches. It also describes how the proposed CoPPGIS will innovate, improve, promote, support and contribute to the existing public participation practices.

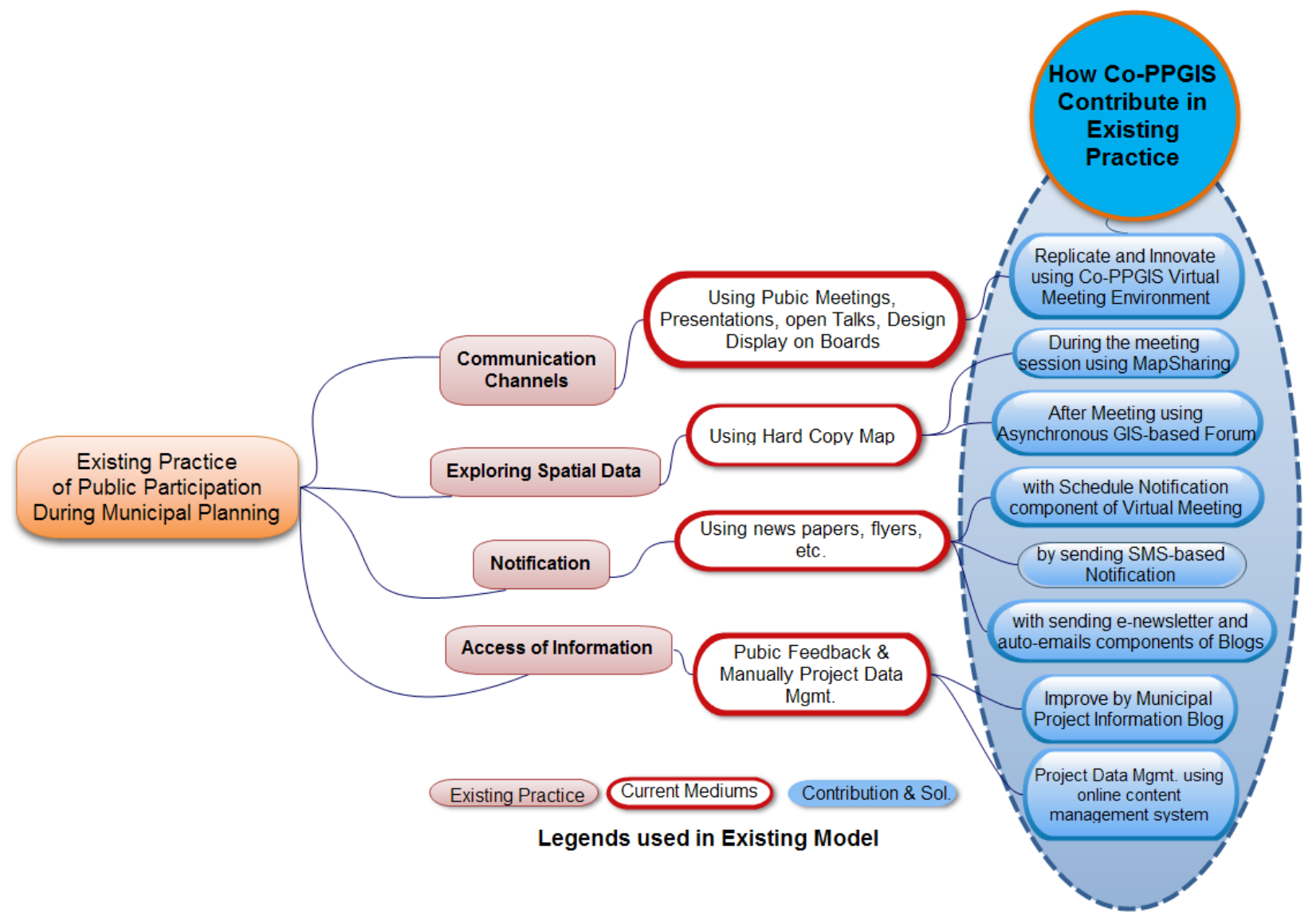

Figure 3.9: Identifying Relation between Existing Participation Practices and the Suggested Co-PPGIS 
Figure 3.9 also shows the research gaps in current communication mediums found during the literature review and suggests how Co-PPGIS contributes to the existing practice to enhance public participation in municipal planning and development projects. This may include:

1) the issue of less effective communication due to fixed-time meeting schedules, accessibility issues, lengthy presentations and open talks with authorities can be complemented by using a Co-PPGIS meeting environment that provides anywhere/anytime/anyone accesses with real-time participation support;

2) the less effective way of exploring spatial data in the meeting sessions currently being used (i.e., using hard copy maps) can be improved either by a spatial component of a GIS-based forum or by a real-time map-sharing collaborative component of the Co-PPGIS, which enhances the degree of public participation along with spatial data exploration during the real-time virtual meeting sessions;

3) a less effective procedure of sending notification related to ongoing municipal development projects can be automated using the meeting scheduling/notifying and/or the e-newsletter components of the Co-PPGIS blog;

4) access to information related to a municipal project's level of data can facilitate using the project information blog, which displays the project's notice detail, minutes of the meeting, presentation, document, location, and all useful information related to ongoing or future municipal projects; and

5) the lack of support to quick decision-making can be promoted using Co-PPGIS. It improves public participation or input as well as helps disperse decision-makers to work concurrently on a real-time basis to finalize the decision in a timely manner, which ultimately reduces the time span of planning as well as chance of failure.

In brief, access to the information required for public input, physical presence in public meetings, and well-designed technical presentations are major factors affecting the efficiency and effectiveness of public meetings during municipal planning.

\subsection{Identify Direct Relationship between Arnstein's (1969) Ladder of Participation and Co- PPGIS}

Figure 3.10 identifies the relationship between the level of participation practices and the proposed Co-PPGIS. It shows the relationship between a common set of prototype components of Co-PPGIS and the degree of public participation based on Arnstein's ladder (discussed in Section 2.1.2), which shows the exchange of information at different participation levels (i.e., citizen power, tokenism, and non-participation, etc.) between the public and decision-makers. 


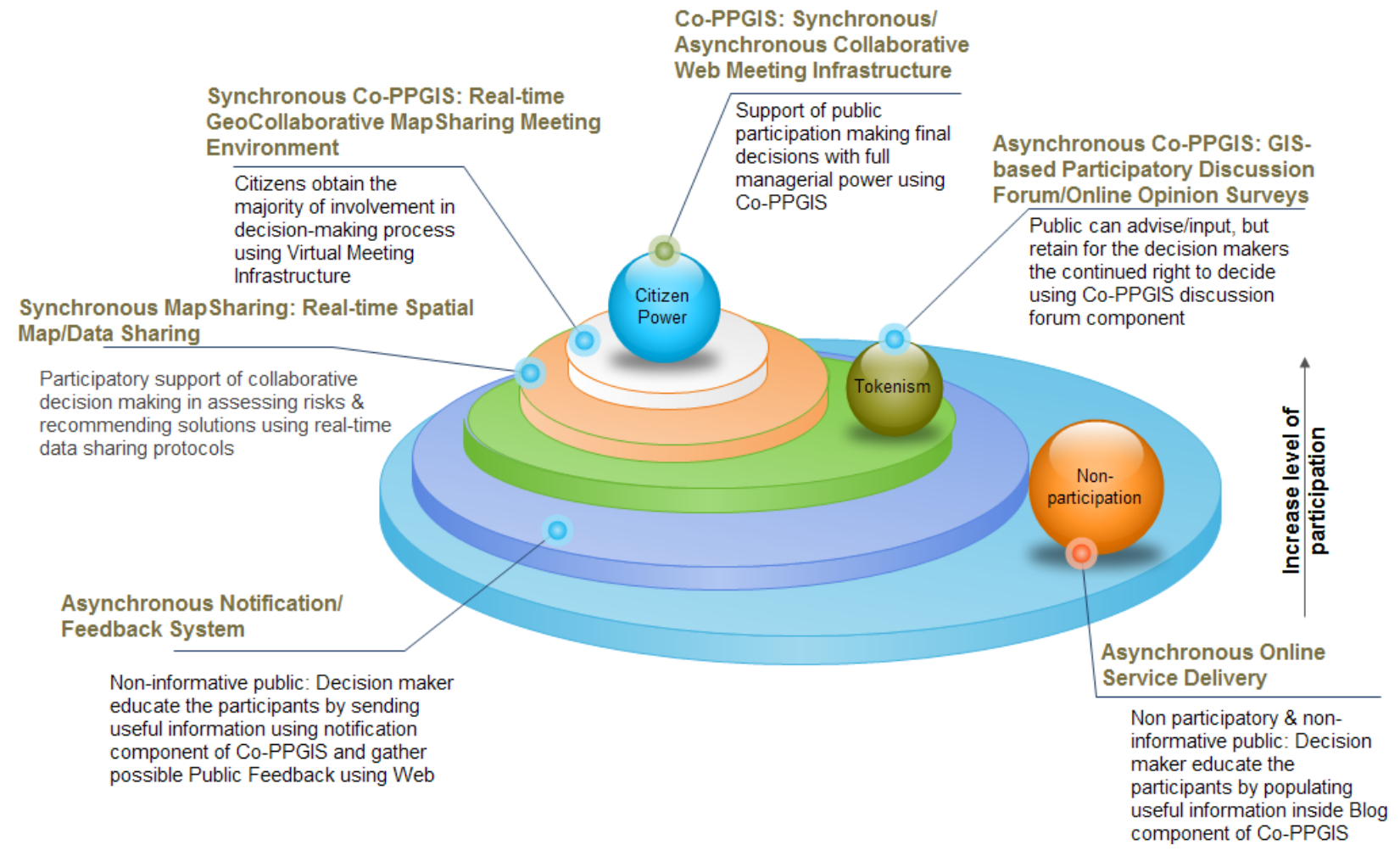

Figure 3.10: Modified Arnstein's Ladder Applied to Co-PPGIS Components (Butt and Li 2014)

Figure 3.10 illustrates that each public participatory level of Arnstein's ladder has a direct relationship with one or more Co-PPGIS components (i.e., notification, GIS-based discussion forum, real-time map-sharing, and virtual meeting environment). These components are designed and developed to contribute in raising public participation at each level of the municipal planning processes. Every component is created in relation to a rung in a ladder that gives rise to public participation with each step upward. Climbing up to the next level of a ladder enhances the degree of interactivity and participation. The top rung of the ladder represents citizen power where the participation among stakeholders becomes real-time and is facilitated through a more synchronized way of participation using the real-time map-sharing, spatial data and information sharing mechanisms supported by Co-PPGIS. The bottom level of the ladder represents the online delivery of public services through a notification/feedback component (e.g., sending announcements and/or informing the public about upcoming events related to the ongoing projects). This level refers to an asynchronous mode of Co-PPGIS. 


\subsection{Identify Direct Relationship between Weidemann and Fermers (1993) Ladder of Participation and Co-PPGIS Components}

The open geo-processing standards, collaborative participation and access to quality of open geospatial data are essential requirements of PPGIS development for planning and management-related processes. PPGIS and/or Geospatial tools ensure access to up-to-date quality data and instant planning in order to increase the degree of public participation, and ensure rapid decision-making during the planning and development process. In Western countries, the general public and decision-making authorities are the key stakeholders in any planning and development situation and require up-to-date and quality information prepared at a different level from all participants of different situations. This helps them make the best decisions among the possible alternatives in a municipal planning situation.

In municipal planning situations, stakeholders with different backgrounds, ideologies, and work styles are involved in each phase of municipal development life cycle (Cutter 2003). Often, it is difficult to gather the concerned departments on a single platform to perform multiple tasks simultaneously. Fortunately, Co-PPGIS provides a virtual meeting place to deal with these difficulties. For example, a common set of mechanisms developed to support real-time Co-PPGIS help in this regard by involving all the concerned agencies in all steps of public participatory planning related activities efficiently. Co-PPGIS components are developed to enhance the decision-maker's capability to deal with complex problems and implement better plans for small municipalities that have fewer resources to purchase expensive license-based PPGIS applications. Moreover, Co-PPGIS components enable the exchange of information gathered from multiple sources to keep all departments up to date, which is a necessary requirement in the planning and development situation.

In Section 3.5, Figure 3.10 shows the relationship between Arnstein's (1969) ladder of participation and Co-PPGIS components. Similarly, Figure 3.11 illustrates the pyramidal relationship between a common set of participatory components of Co-PPGIS and the public participation ladder (see Section 2.1.2) of Wiedemann and Fermers (1993), which enables the exchange of knowledge at different participatory levels between public officials and decision makers. 
Figure 3.11 shows that each component of Co-PPGIS has a direct relationship with the step of a Wiedemann and Fermers ladder.

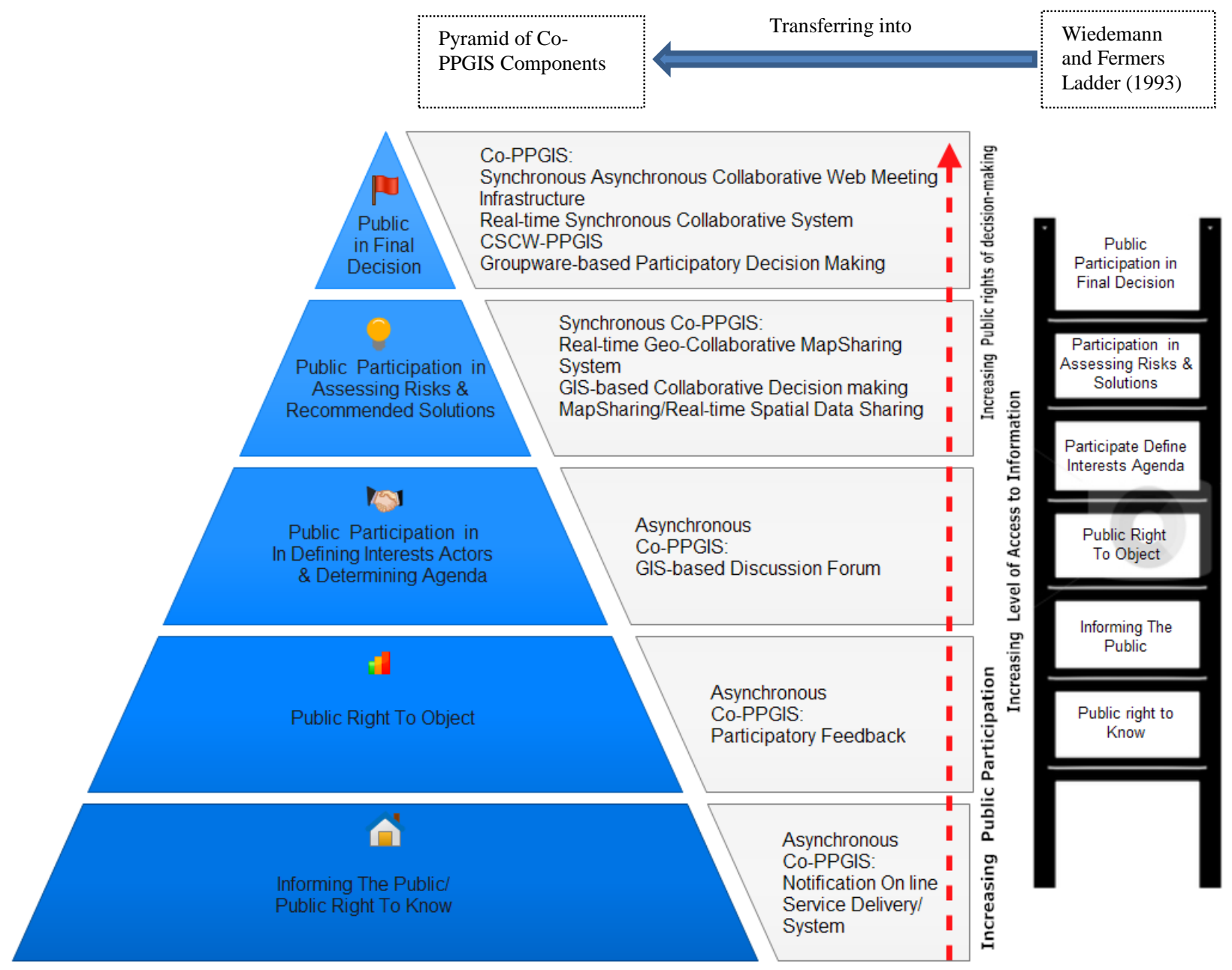

Figure 3.11: Wiedemann and Fermers Ladder (1993) Applied to Co-PPGIS Components-pyramid which enables the Exchanging of Information at Different Participation Level among Public and Decision-maker

It also depicts that how Co-PPGIS components-pyramid and the ladder both provide rise to public participation, increase access of information and provide public rights to decision-making as you ascend the pyramid. Co-PPGIS creates a link between collaborative participation and decision-makers and the public during Planning and Development (P\&D) workflows. Co-PPGIS infrastructure may provide a number of accomplishments to keep all the decision makers up-to-date by: a) creating status reports on construction project based upon the information collected from multiple sources, b) integrating informational exchanges, c) maintaining the list of participants 
involved in the diversified planning situations in order to utilize the available resources in a collaborative way, and d) preparing different scenarios before, during and after public participation to develop a better understanding for sharing up-to-date information and making better decision by minimizing the time factor.

There are several scenarios where planners, municipal bodies, and the public are working collaboratively investigating comprehensive information and knowledge-driven approaches. For example, Co-PPGIS scenarioanalysis tools can be used: 1) to find a health facility with buffer analysis to implement new neighborhood strategic planning, 2) to highlight a high crime area, 3) to explore new approaches to housing redevelopment, and 4) to find the best park location in the nearest neighborhood. Fortin (2008) states that the public, as one of the important stakeholders, requires: a) rapid access to a base map of those areas where the municipal amendment takes place with simple communication tools, and b) geo-collaboration tools for accessing/exploring spatial information of particular municipal project's data. All of these requirements can be facilitated using a Co-PPGIS infrastructure. All the key stakeholders can play their role in the situation rapidly and effectively, if the required information is provided to decision-makers and the public at the right time. In addition, the degree of poor decisions can be reduced with better planning.

\subsection{Methodology Adopted for Requirements Engineering of Co-PPGIS}

Requirements Engineering (RE) methodology is the stepping stone of all the later Software-Development Life Cycle (SDLC) stages in software development, but little research has been done to investigate the appropriateness of the available elicitation and analysis techniques in the domain and context of an online real-time geo-collaborative public participatory system. The absence of research and standardized RE process is more noticeable in the case of PPGIS applications that are employed during municipal planning. The general practice is to use a single technique for all applications irrespective of their domains. The selection depends on the analyst's familiarity with the technique instead of the system requirements. In recent decades, the WWW has made great advancements and has a huge scope and potential for use with real-time public participatory systems. Unfortunately, not enough research has been provided; and therefore, more conceptual and empirical research work is required for the online context of the system.

\subsubsection{RE Process of Co-PPGIS}

Jarke and Pohl (1994) defined a requirement engineering process as "A systematic process of developing requirements through an interactive co-operative process of analyzing the problem, documenting the resulting observations in a variety of representation formats, and checking the accuracy of the understanding gained". In requirement engineering, UML plays an important role during the analysis and design of object-oriented system 
such as GIS-based collaborative system and/or real-time PPGIS. It can be used for all stages of the development life cycle and across different implementation technologies.

Li et al. (2006) states "the municipality or city planning and development process is an extremely complex decisionmaking process" and the Object-oriented Software Engineering (OOSE) is an effective technique to manage the complexity of this process. It is true in this regard that the usage of OOSE, UML and Computer Aided Software Engineering (CASE) tools during the RE and Requirement Analysis (RA) of a Co-PPGIS infrastructure will result in a more effective and reliable outcome. Thus, all building blocks of the system life cycle will be recognized using object-oriented software engineering.

A real-time Co-PPGIS is meant to allow both synchronous and asynchronous communication during planning and development-related process workflows. If users are not able to participate using a real-time map-sharing environment, and/or using GIS-based forums, the system will not pass the acceptance criteria. For Co-PPGIS to be considered complete and in accordance with the needs of the users, both synchronous and asynchronous collaboration functionality is necessary. In addition, properly defined requirements in an effective RE process also lead to: a) faster development (since later stages can advance quickly); b) higher satisfaction (less rework and issues); and c) agreed and announced acceptance criteria (higher rate of completion). 
Figure 3.12 illustrates the general workflow of Co-PPGIS requirements engineering and analysis.

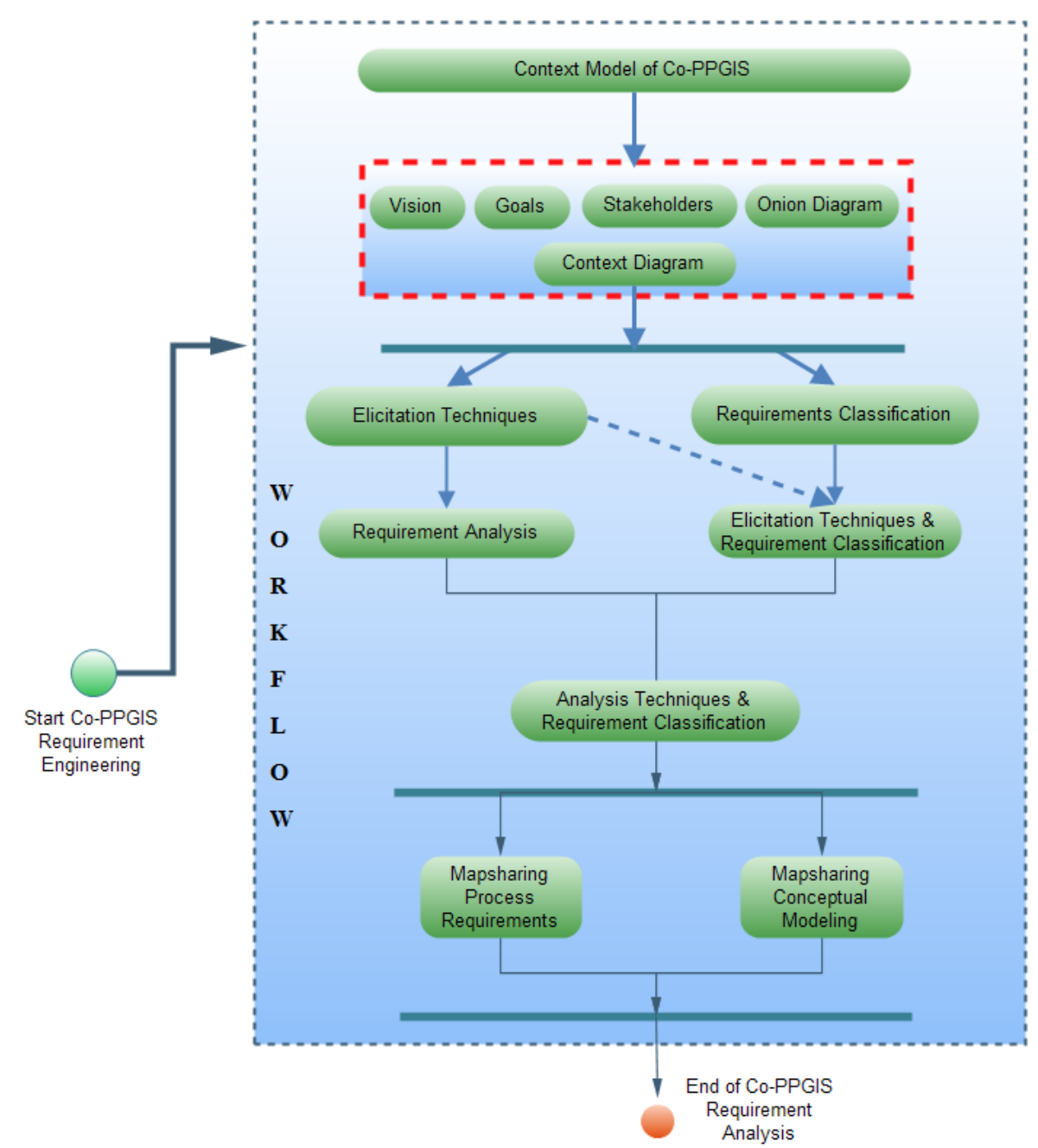

Figure 3.12: Workflow of the Requirements Engineering Process for Co-PPGIS

The context model of Co-PPGIS RE workflow simply describes its attributes starting with the vision, the goals and a list of stakeholders affected by the suggested system infrastructure that are necessary to develop the context of CoPPGIS. The context model is an essential element of RE that may also be used more widely in finding the functional requirements discussed in next section. As discussed earlier, identifying gaps in the existing municipal planning process workflows is also helpful in modeling the context of the Co-PPGIS, which ultimately generalize in the form of capability requirements. The RE context model can be defined in five elements which include vision, goals, 
stakeholder analysis, onion model and context diagram (see dashed area at the top in Figure 3.12 describing elements of context model).

Once the system context is defined, elicitation techniques based on UML, GIS and Web concepts are formulated, followed by a requirement classification, which highlights a set of requirements most important to a Co-PPGIS application. The elicitation techniques are linked to the requirement classification model. The work done on elicitation and requirements contributes to RA. Applying requirement analysis techniques is the next step in the RE process after gathering the initial requirement of the Co-PPGIS. The RA focuses on behavioral modeling diagrams, which are represented using sequence diagrams and are useful in explaining, elaborating and analyzing the CoPPGIS application components. A sequence diagram is a form of an interaction diagram, which is the most popular UML artifact for behavioral modeling and focuses on identifying the behavior within Co-PPGIS components. Appendix Six describes sequence diagrams for Co-PPGIS map-sharing component services.

\subsubsection{Co-PPGIS Context Model}

The context model incorporates the major components of the RE process and initializes using the formulation of vision statement, finalizing goals, stakeholders, onion and context diagram.

\section{a) Vision: Initialization of the RE Process}

The vision provides the earliest inspiration for the creation and development of a new system infrastructure. It is usually the first insight of the system and also ignites the requirement elicitation process by laying down a concept in the form of a vision. Furthermore, the vision is supplemented with goals and abstract ideas (Jarke and Pohl 1994), eventually describing the entire system infrastructure. The research suggested infrastructure for an online synchronous collaborative public participation system is stated below as an example of a vision of a PPGIS system, emphasizing public participation during municipal planning and management-related workflows.

\section{b) Goals:}

Requirement engineering is also described as a process which realizes system goals into a workable solution. Some of the goals of an online public participatory system include:

- Co-PPGIS recognized as a public participatory system that is democratic (use bottom-up approach), with a non-differential access to tools and technology for direct and effective user input.

- An online GIS system that incorporates tools and technology, which is usable and accessible to all users irrespective to their background and expertise, without the need of guidance by a specialist. 
- Using the latest Web 2.0 technology to provide dynamic and interactive rich media content, with a user centric approach for easy access to GIS-based services in synchronous and asynchronous modes and blog/forum-based discussion posting (asynchronous communication) (Roche et al. 2012).

- A public participatory system that effectively and efficiently provides support during the decision-making processes, with the level of participation and influence by stakeholders for balancing within the resource and time available to complete the proceedings (Schwedes and Werner 2010).

- A real-time participatory system that provides services to its users:

- Participatory: co-browsing, screen sharing, map-sharing, conferencing, feedback, polling, whiteboard, file uploading

- Communication Channels: email, virtual meeting, notification, focus group discussion, observation sheet, chat, social media, and public access to information to ongoing, new and completed projects through a common interface. Virtual e-meetings and discussion rooms to support municipal planning workflows are also examples.

- Multimedia: audio, video, webcam

$\circ$ GIS: resource maps, navigation tools, spatial and attribute data

- Administration: configuration and content management through Web

\section{c) Stakeholders:}

Co-PPGIS application has many diversified stakeholders, involving situations where stakeholders need to constantly interact, negotiate and make collaborative decisions. These decisions, in turn, can have a significant effect on a large community and general population. The diverse nature of stakeholders make it necessary that all participatory systems have a thorough user's analysis, with timely identification of all potential stakeholders and the affected systems (Schwedes and Werner 2010). Besides knowledge of all the relevant stakeholders, it is also important to identify the right stage and time, as well as the right level of stakeholder participation, in the process (discussed below in an onion diagram).

In UML, an actor (internal and external) specifies a role played by a user or any other system that interacts with the subject. For example, the maps provided by Google and other open mapping sources provide satellite imagery and street maps that act as base layers (external actors) of the system. Various Co-PPGIS components interact with this base layer to provide real-time map-sharing, collaborating web meeting infrastructure and a GIS-based discussion forum. 
Some of the internal actors of the system are as follows:

- Experts Users: moderator, super moderator

- Admin: for configuration, website and user management

- General public: the grass-root level masses (general public participants, ordinary citizens)

- Municipal and community planning authorities: domain experts, municipal bodies, environmental assessment, contractors/developers, NGOs, and researchers

- Development team: developers, managers, DBA

\section{d) Onion Diagram:}

The onion diagram (suggested by Alexander and Robertson 2004) distinguishes which stakeholder directly interacts with Co-PPGIS. The innermost circles show the most interaction with the system in term of participation, policy making and decision-making processes, while the outer rings progressively become more general (Schlossberg 1998). An onion model with the onion rings represents the placement of stakeholders with a Co-PPGIS application. The rings are built based upon stakeholders' ability to influence the system and/or to describe the relationship between the system and the stakeholders (Butt and Li 2015). Considering these criteria, an onion model is developed to illustrate the stakeholders who have primary interactions or strong relationships with the Co-PPGIS. Figure 3.13 shows an onion model with the onion rings, which represents the level of interaction of stakeholders with a CoPPGIS application.

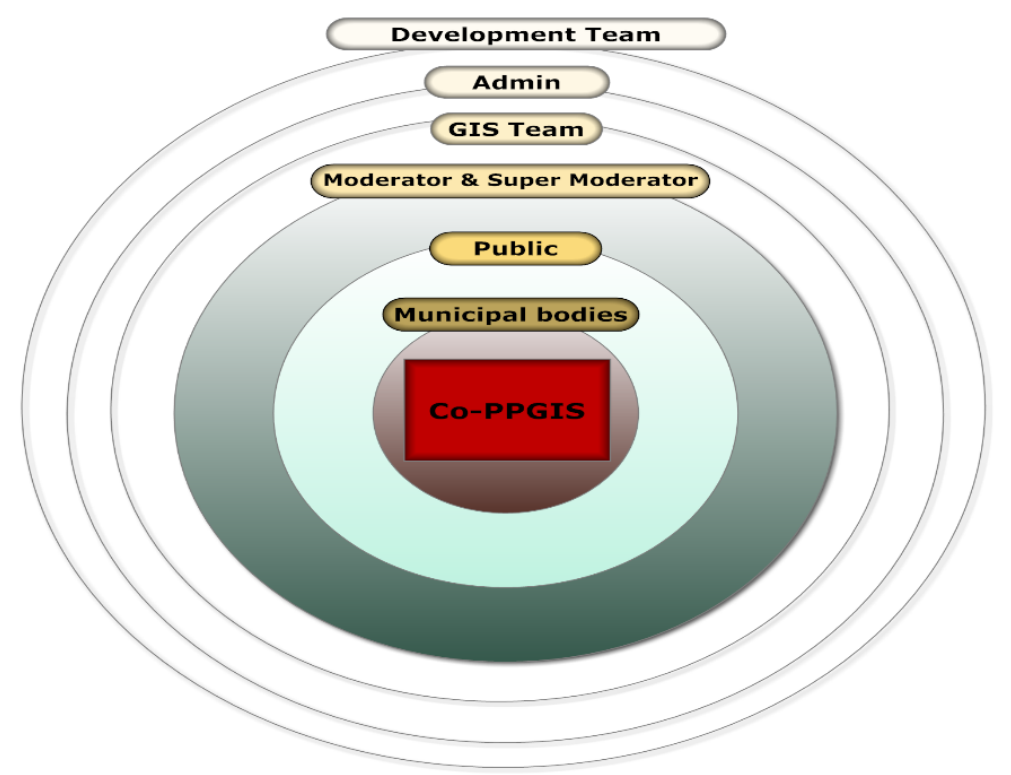

Figure 3.13: Co-PPGIS Actors (Stakeholder) Onion Model Rings Show Stakeholders' Ability to Influence the System in Terms of Their Level of Participation and/or Decision-making Processes 
The municipal planning authority, which has the responsibility of formulating laws and the final policy, has the positive influence (strongest relationship) on the system. Next comes the general public, who despite lacking the knowledge to make an effective municipal plan, are nonetheless important because they are the beneficiaries/end users of the system. Then, come the general participants (i.e., moderators, super moderators or others), who act as facilitators and do have some interest in the system. The GIS team, which sends information of the actual municipal plans to be displayed on maps and used in the system, come next. Then, the administrator, who configures and controls the web-based settings. Lastly, the development team, which essentially has the function of developing a system thoroughly explained through the requirement engineering process.

\section{e) Context Diagram:}

Initial inputs of RE (vision, goals, stakeholders and onion model) are combined to build a model of the system using a context diagram. A context diagram places the system as a black box and relates its functions for interactions with the outside world. It depicts how the data is exchanged between external users and the system like the proposed CoPPGIS. Considering the stakeholders and the inputs/outputs of Co-PPGIS, Figure 3.14 displays the context diagram with major functions, participants' participation and external data source identification.

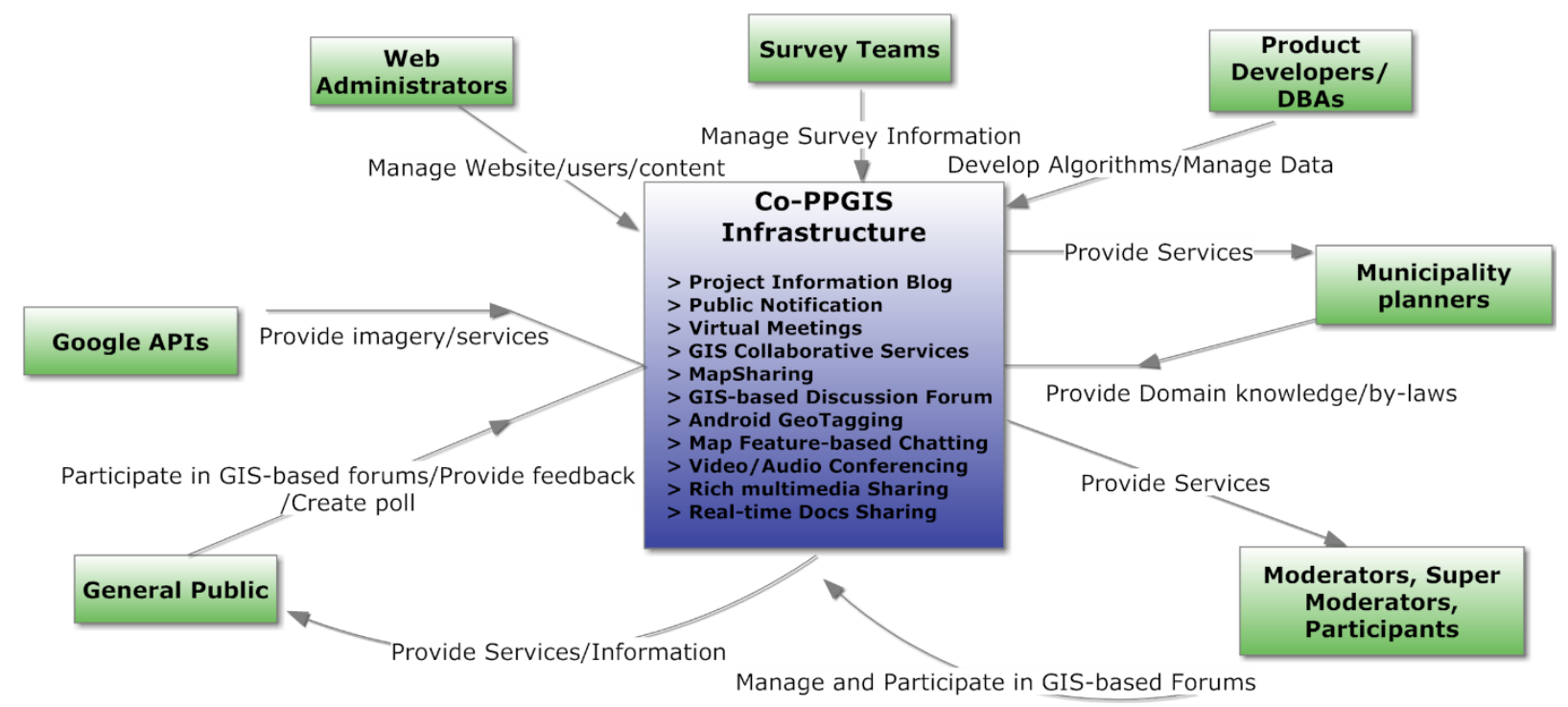

Figure 3.14: Context Diagram of the Co-PPGIS (Butt and Li 2012) 


\subsubsection{Functional Requirements of Co-PPGIS}

The functional requirements of Co-PPGIS are identified based on a literature review, the need of existing planning practices, dialogues with municipal planning bodies, and an evaluation of the current PPGIS frameworks, as well as defined by following the RE processes. The functional requirements are discussed briefly as follows:

Collaboration: A Co-PPGIS must allow direct collaboration between a participant and Co-PPGIS components through its user-friendly Web interfaces. In addition, the participant must be able to create, add, delete, save, update or modify, and view spatial and non-spatial information related to a municipal project.

Computation: Many municipal planning and development related issues need thorough computation to assess a large number of potential alternatives. Co-PPGIS computation tools need to be designed in a way that can provide an "initial" point to its end-users (i.e., the public and stakeholders), who can then develop new choices and alternatives to replicate their personal preferences. For example, implementing a heat map analysis tool in the Co-PPGIS infrastructure, in order to find out which areas of city are crowded or which areas have more criminal activities.

Collaborative Sharing: Municipal planning is a complex task requiring spatial data and real-time collaborative data sharing. In addition, the Co-PPGIS system needs to be designed by keeping in view stakeholder needs and requirements. As discussed earlier, PPGIS applications dealing with municipal planning have multiple diversified and varied stakeholders with different backgrounds and objectives. A Co-PPGIS should be developed to consider the preferences of stakeholders, who often have diverse cultural, political, and social backgrounds. For example, communication in the same language is required when a group of people are discussing or sharing thoughts. A CoPPGIS must be able to accommodate these kinds of amendments.

Intended Use: Co-PPGIS needs to be developed with the objective that the system will be used by the general public and other stakeholders (as discussed earlier). Highly usable interfaces are required to make this application usable even for non-experts.

Effective and Fast Communication: The users of Co-PPGIS should be able to brainstorm ideas, as well as present and exchange them. The system should provide the up-to-date, accurate and precise data for the area under discussion. Effective and fast communication among all stakeholders is required to ensure that the collected and stored information is disseminated to all those concerned within a timely period. 
Interactive Discussion: The Co-PPGIS should enable interactive discussions with map-sharing mechanisms and tools, aiding in better visualization, enhancing public participation, and better decision-making. Focused virtual meeting rooms, with defined participant roles and a democratic, friendly, and non-chaotic environment, should exist for an effective solution.

Interoperable System: In order to make a Co-PPGIS interoperable, standards need to be followed. For example, system achieving high performance, with the addition of hardware scalability, increases its capacity as a result of the addition of new hardware. Since the system will be used concurrently by potentially many users, the architecture needs to be scalable in order to provide acceptable performance when the load increases after a certain level. In addition, in case of implementing an interoperable solution during prototype development, Co-PPGIS components need to be developed and implemented as: 1) online Web-based client server architecture, based on interoperable map services (e.g., OGC KML service), which can be used in a map-sharing component and configured in both CSPE and CAPE to provide interactive virtual tours related to municipal planning related projects, 2) OGC open standards-based components (e.g., Co-PPGIS map-sharing component WMS standard) provide a simple Hypertext Transfer Protocol (HTTP) interface for requesting geo-registered map images from one or more distributed geospatial databases, 3) OOSE modular approach that was adopted during the Co-PPGIS components development, in which each component can work independently, integrated easily with others and reused for the further development, and 4) Human-Computer Interaction or HCI design approaches to make online user-friendly and interactive interfaces.

The functional requirements form the basis of the proposed design and architecture of the underlying system. They are more important as changing design and architecture of the system will require a complete overhaul of the underlying system. In order to satisfy the above dimensions, major functional requirements are further elaborated upon in term of Co-PPGIS functional requirements. 
Figure 3.15 summarizes some of the functional requirements for participants and/or stakeholders with respect to the

\section{Co-PPGIS.}

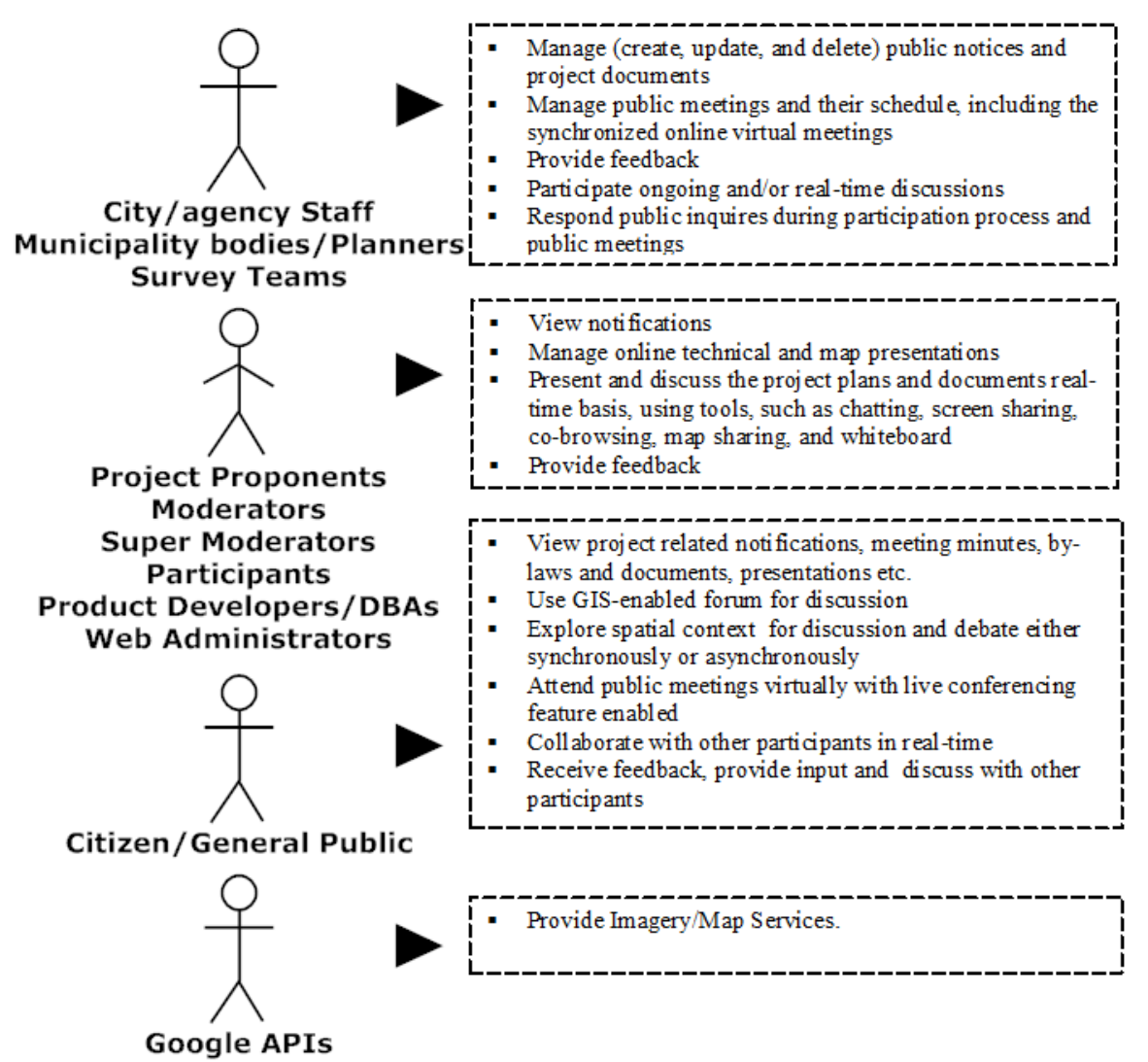

Figure 3.15: List of Functional Requirements for Participants

Figure 3.15 shows how different stakeholders interact with the system. For example, project proponents can view notice boards for public meeting information (e.g., public notices, meeting schedule and location). The public provide electronic communication support, which may include email, chat rooms, online survey, and threaded discussion forums. The project proponent may require video conferencing using real-time streaming of public meetings along with the support of real-time synchronous sharing of PowerPoint presentations, documents, map displays and chat. The public need a collaborative map-sharing mechanism with necessary GIS functions to allow participants to explore the planning scenarios collaboratively within the required spatial contexts and to enable the public meeting presenters to share their maps during public meetings. 
In addition, the public may need document repository and access support for all project-related materials, including regulations, planning documents, minutes, and presentations. The Co-PPGIS must use a server push technology to do synchronous participation such as real-time instant messaging, map-sharing, and geo-referenced pointing that can "point" at the shared view of a map. The Co-PPGIS synchronous and asynchronous participatory environment should allow more than one participant to use whiteboard function to draw geometry-based incidents; to share georeferenced annotations; to navigate together in the map (e.g., changing map scale and position, etc.) and to switch map view among different base map layers (e.g., street map, satellite, hybrid and terrain). The Co-PPGIS should provide easy access (e.g., participants can access the main interface of a prototype by using any browser such as Internet Explorer, Firefox, and Chrome).

\subsection{Co-PPGIS High-level Architecture and Flow Models}

This section describes the system architecture and design, which is a composition of some individual integral components that are used to develop the proof of concepts. Figure 3.16 illustrates a higher-level architecture of CoPPGIS. Based on this concept, Co-PPGIS supports not only a form of asynchronous participation, but also of realtime synchronous participation over the Internet in municipal development projects that are undergoing planning and require public participation. The Co-PPGIS is composed of different subsystems that are facilitated by a different group of participants to support a continuous public participation processes during municipal planning. Each of these subsystems may fulfill several capability requirements using different technology. For example, subsystems in accordance with participation may include an online GIS-based participation forum, audio/video conferencing, rich multimedia sharing, and real-time map-sharing. A Co-PPGIS introduces the concept of a realtime map-sharing mechanism to online public participation, collaboration and communication with the support of a Web-based GIS and a spatial database. Other subsystems, such as spatial components and blogs may serve various functions during the overall municipal planning process. The Co-PPGIS blog component manages several municipal projects within the same situation so that each municipality can have a single blog interface for the public to participate in all related projects open for public participation. 
The high-level architecture of Co-PPGIS is shown in Figure 3.16.

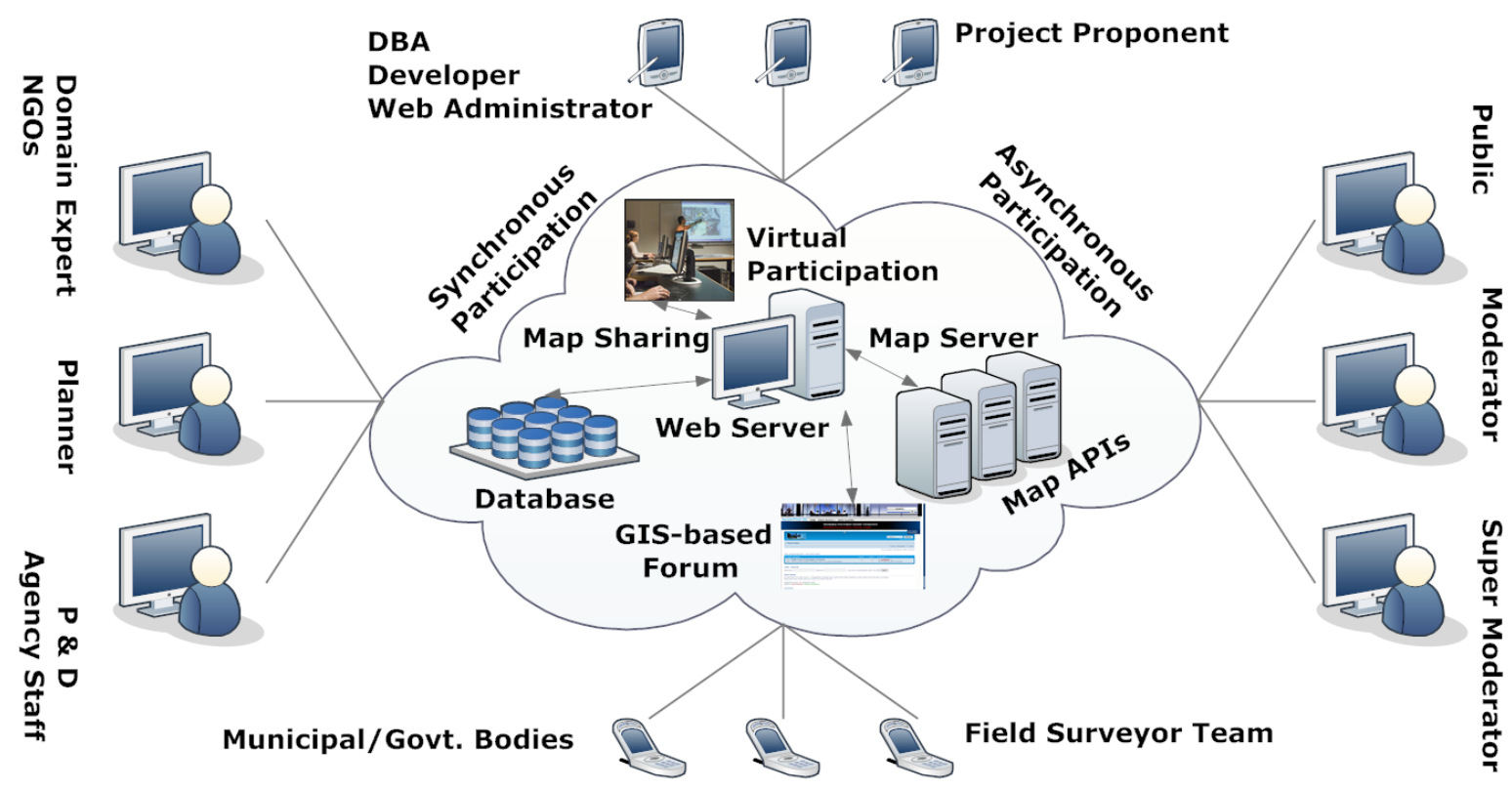

Figure 3.16: High-level View of Co-PPGIS

This conceptual architecture of Co-PPGIS shows that multiple participants (e.g., project proponents, local citizens, NGOs, domain expert, other community leaders, and planning and development agency staff - city staff, local planning staff, regional planning staff and other agency staff) are collaboratively involved and may have different requirements in terms of access to information, level of participation, and need for collaboration. Figure 3.17 shows a use case model of Co-PPGIS, which represents the external view of the prototype components by introducing and involving its main stakeholders. The use case model depicts the proposed components of the Co-PPGIS and shows communication between the participants and components. For instance, the model is categorized into two major use cases (i.e., CSPE and CAPE), which are recognized for understanding user dialogue with Co-PPGIS components and depicts the functionality that are built in the Co-PPGIS. 


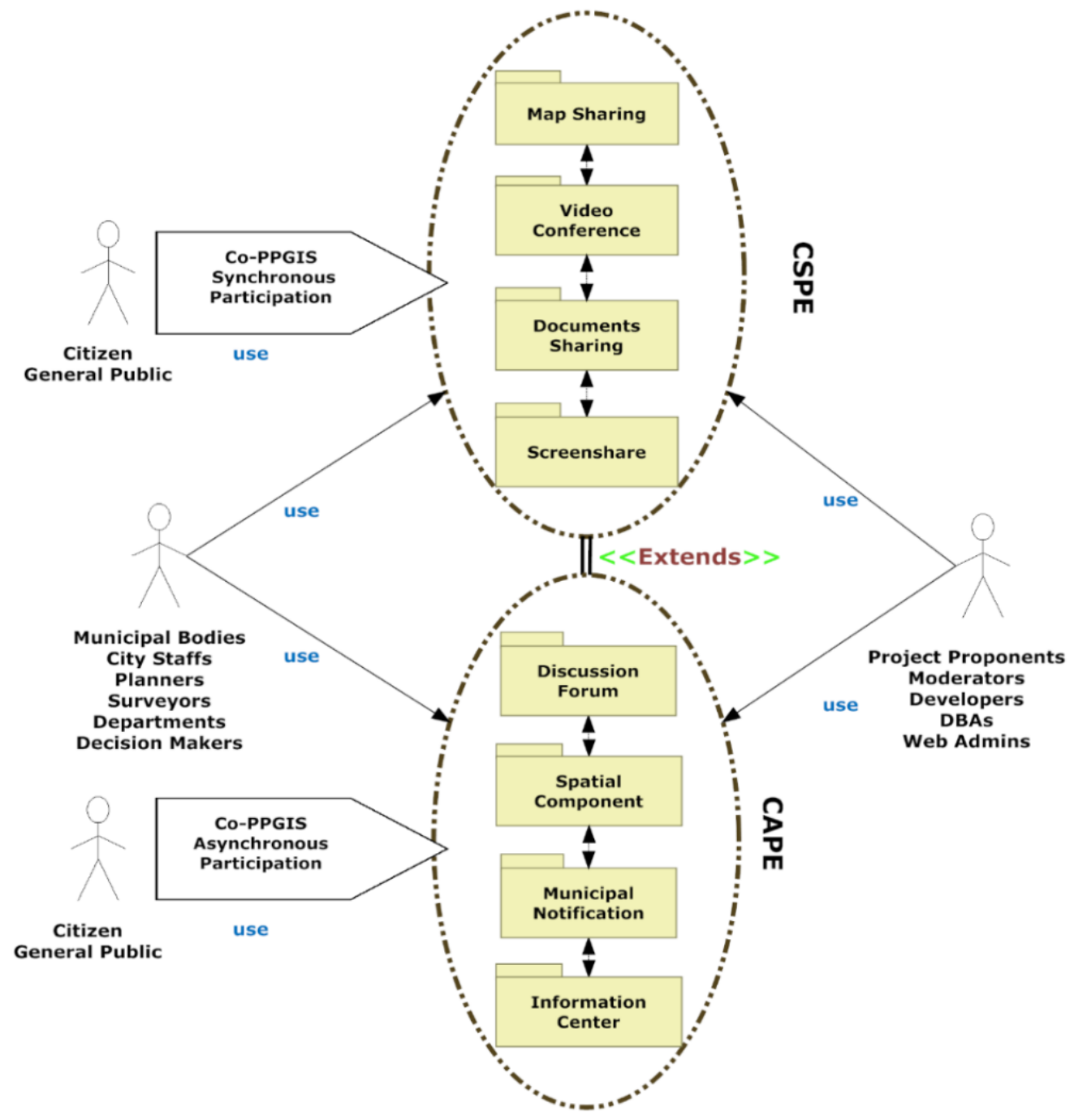

Figure 3.17: Use-case Model of Co-PPGIS

As discussed earlier, the planning and participation process of municipal planning and development projects normally involves different groups of participants. Figure 3.17 shows the Co-PPGIS involves group of participants by providing interaction with its components using a synchronous and asynchronous collaborative environment, which is developed to facilitate public participation and better decision-making.

The context diagram (discussed in Co-PPGIS context model in Section 3.7.2) is enhanced and elaborated in the context Data Flow Model (DFM) level 1, where functions related to the Co-PPGIS application are described. 
Figure 3.18 discusses the CSPE and CAPE major functions in the form of a data flow model that has two major modules, the main application and the admin module. Admin administer, the main interface and the users, whereas the main Co-PPGIS application allow users to sign in, participate in a meeting, use geospatial tools, and explore multimedia facilities with other participants.

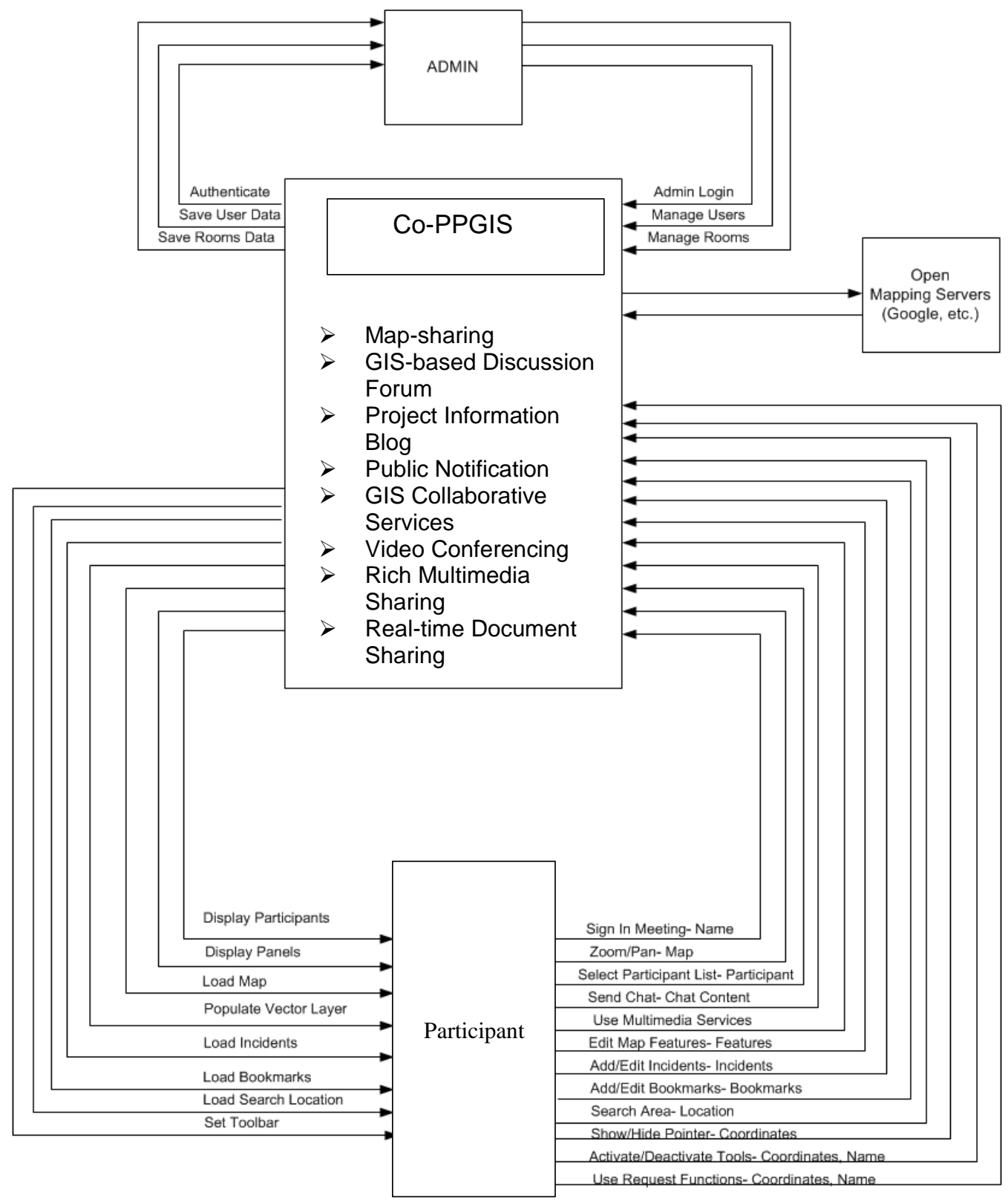

Figure 3.18: Co-PPGIS Main Functional Processes in the Form of Data Flow Model 
The context data flow model is further elaborated at level 2 of data flow model, with some of the inner processes highlighted. The processes and interfaces are included as a base map module, map services, admin, and chat modules and are accompanied with a logical description of data type is being stored (e.g., users, chat, geo-spatial, multimedia, and map features, etc.) (See Figure 3.19).

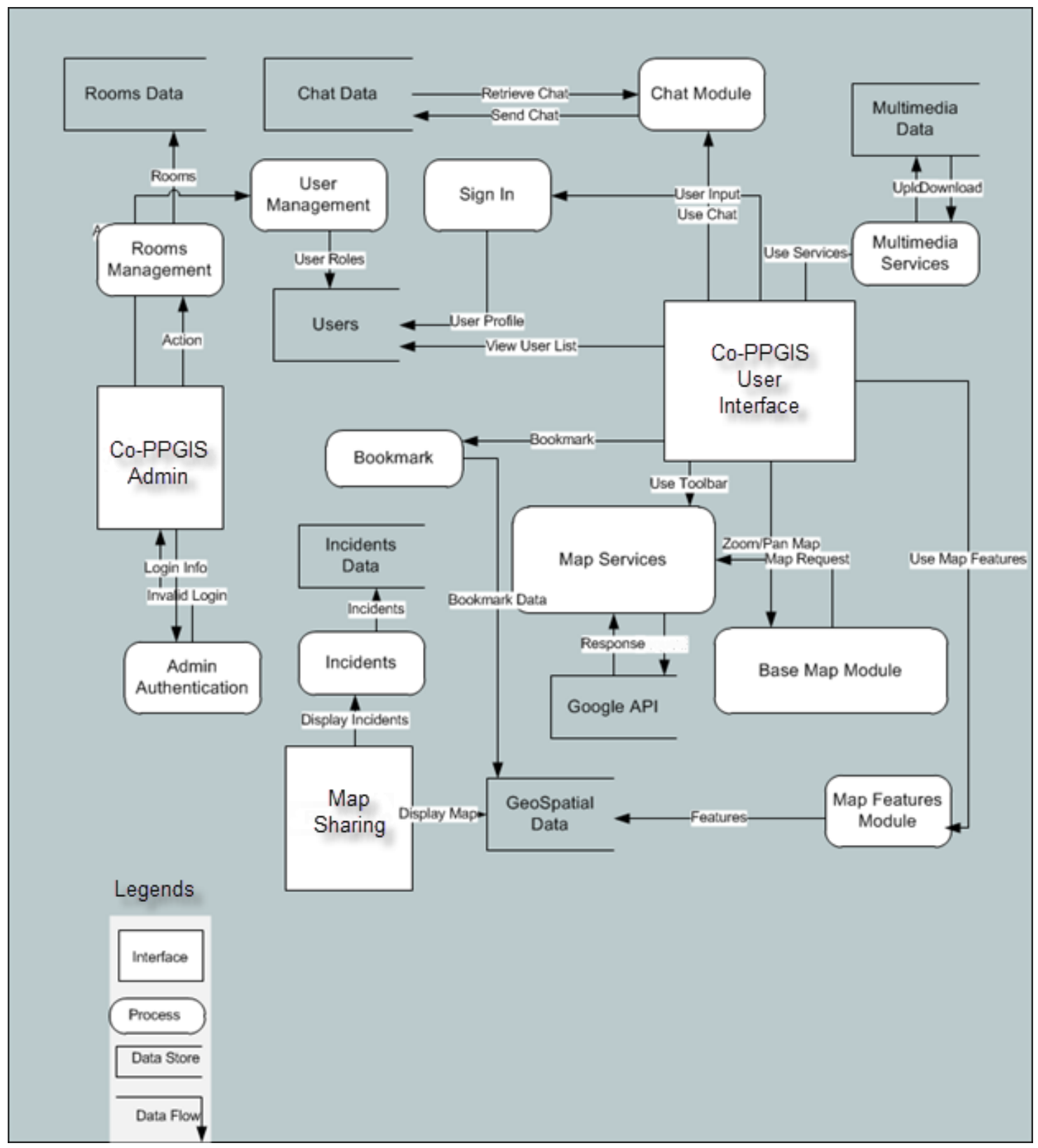

Figure 3.19: Co-PPGIS level 2 Data Flow Model 
These major functional processes are further elaborated and described in relation to their technology management, integration, and implementation context later in Section 4.3.

\subsection{Summary}

This chapter starts with a brief introduction of the Co-PPGIS concept by highlighting its significance during municipal planning and development, followed by a detailed understanding of the Co-PPGIS infrastructure. Gaps in existing municipal planning practices and possible improvements using Co-PPGIS are discussed, followed by higher-level architecture and the requirement engineering workflows, which explain the context model, starting with the vision of the system, the goals and a list of stakeholders affected by the Co-PPGIS infrastructure. The next chapter discusses the development and implementation aspects of the Co-PPGIS prototype. 


\section{Chapter 4 Prototype System Development}

This chapter discusses the Co-PPGIS prototype in terms of how the proposed prototype is implemented and realized by displaying the walkthrough of the user interfaces developed. The prototype Web interfaces are designed for the proof of concept to support Co-PPGIS as well as to realize the analysis and design model. This chapter consists of eight sections. Co-PPGIS brief overview, major participatory approaches and their architectures are discussed briefly in the first three sections. Section 4 explains the Co-PPGIS component-level, service-oriented architectures and map-sharing mechanism, which is the main module shared by both CSPE and CAPE. The fifth and sixth sections depict the implementation of the Co-PPGIS prototype components and walkthrough of the map-sharing module, followed by the demonstration of its implementation in planning and management related activities. The seventh and eight sections describe the discussions, problems and issues of prototype implementation and summary.

\subsection{Overview of Co-PPGIS Prototype Application Development}

From a user's point of view, Co-PPGIS is a GIS-enabled virtual environment where one can access information, participate in the planning and decision making process, collaborate with other participants, use discussion forums, attend public meetings virtually, and review the outcomes of the public participation processes. Co-PPGIS is a webbased geospatially-enabled infrastructure that integrates real-time and synchronous/asynchronous map-sharing, chatting, audio/video conferencing, geo-referenced map annotation, and user and meeting management in order to support discussions between multiple users in various geographical locations towards consensus building. The system infrastructure supports real-time integration of data from different sources through web map services APIs and encourages the integration of local knowledge.

The Co-PPGIS prototype is accessible through Web browsers, allowing widespread and easy access to the application through thin client architecture and does not require much technical expertise in the domain of GIS. The component-based architecture, involving objects and server/client side technologies of HTML, JavaScript, JAVA, FLEX and PHP, allows reuse and adaptation of components. Moreover, due to the flexible design and implementation of a prototype modular component of Contents Management System (CMS), Co-PPGIS provides the usability and convenience to the facilitator/moderator for managing Web content and business processes online.

For spatial data integration and retrieval, a PostgreSQL spatial database model (PostGIS) is adopted to obtain a reasonably better transfer rate of the spatial data, which is a big concern while developing any GIS-based collaborative frameworks. 
Open source GIS, groupware and other OSS tools are used to make a cost effective application for smaller budget municipalities. Besides, following the standards enforced by OGC on OSS-based applications, components, and interfaces, result in a better quality interoperable system, which can be integrated with other OSS solutions and customized for future enhancements.

Service-oriented architecture is followed to make a sustainable system. A modular design using components as services is used. New services can be created and enhanced through the integration of current services that make up the composite service chains.

A web 2.0 and object-oriented component-based modeling technique is used to boost the response time and interactivity of the system. Efficiency and quick response are one of the core requirements of a system. The performance issues are determined using the usability evaluation and testing approach that is discussed in Chapter 5 .

\subsection{Co-PPGIS Synchronous Participatory Environment (CSPE) High-level Architecture}

The Co-PPGIS synchronous participatory environment, which supports real-time map-sharing, is designed for a combined action or synergy of multiple components (discussed in Section 4.3). These components are responsible for a variety of unit functions using two tiers of communication protocols. The presentation tier, which runs over Hypertext Transfer Protocol (HTTP), is designed for real-time communication between users/stakeholders (e.g., decision makers) with the help of Web console.

The application tier, which constitutes internal architecture, is based on OSS-based components, modules and services. The CSPE backend architecture is internally comprised of three major components that are linked with each other for two-way communications using Adobe flex framework and JAVA scripting modules. These backend components, along with the HTTP server component, are briefly discussed below.

Real-time Multimedia Collaboration Services: provide the infrastructure that enables developers to add real-time collaboration capabilities (real-time push messaging) to their applications. Collaboration service is a JavaScript Object Notation over a HTTP based system that leverages Web 2.0 messaging and an interaction paradigm (Lemos 2006). It is usually commoditized by using a Red5 open source media server that uses a real-time multimedia protocol to support audio/video streaming (Wang and Xu 2010). 
Blaze Data Service (BlazeDS): provides real-time Web messaging capabilities (a complete publish/subscribe infrastructure allowing Flex clients and the server to exchange real-time messages) for flex-based Rich Internet Applications (RIA) using an ActionScript message format over HTTP.

Web Mapping Modules: handle the user's request on the maps displayed on users' screen by producing open map APIs (Google, Yahoo, and Bing). The synchronous behavior of maps is created to enable co-browsing (co-shared maps) among the participants' Web console by using an Adobe Flex framework, map API for flash and Action/Java script programming language. In addition, the open source Flex software development kit provides an integration link between real-time collaboration services and map APIs modules for the development and deployment of CSPE.

HTTP/ Web Server: handles the user's requests sent from a client-end Web interface (i.e., Firefox or Internet Explorer) to the Web server (Apache Tomcat) and then response back (i.e., an interface along with real-time mapsharing functionalities) to the Web client using HTTP protocols.

The enabling technologies required to develop CSPE are listed and described briefly as follows:

- Server side programming (Macromedia Extensible Markup Language/ Flex Server Script, PHP, JSP)

- Client side programming (HTML, JavaScript, Dynamic HTML, ActionScript, XML, Ajax)

- Web map server and tools (GeoServer)

- Database server (PostgreSQL/PostGIS)

- Real-time messaging server (BlazeDS)

- APIs (Flex SDK, Google map API V3, OpenLayer, GeoEXT v3.4, JQuery, EXTJS)

- HTTP/Web servers (Apache Tomcat) 
Figure 4.1 depicts the higher-level architecture in which synchronous-based participation takes place among the stakeholders.

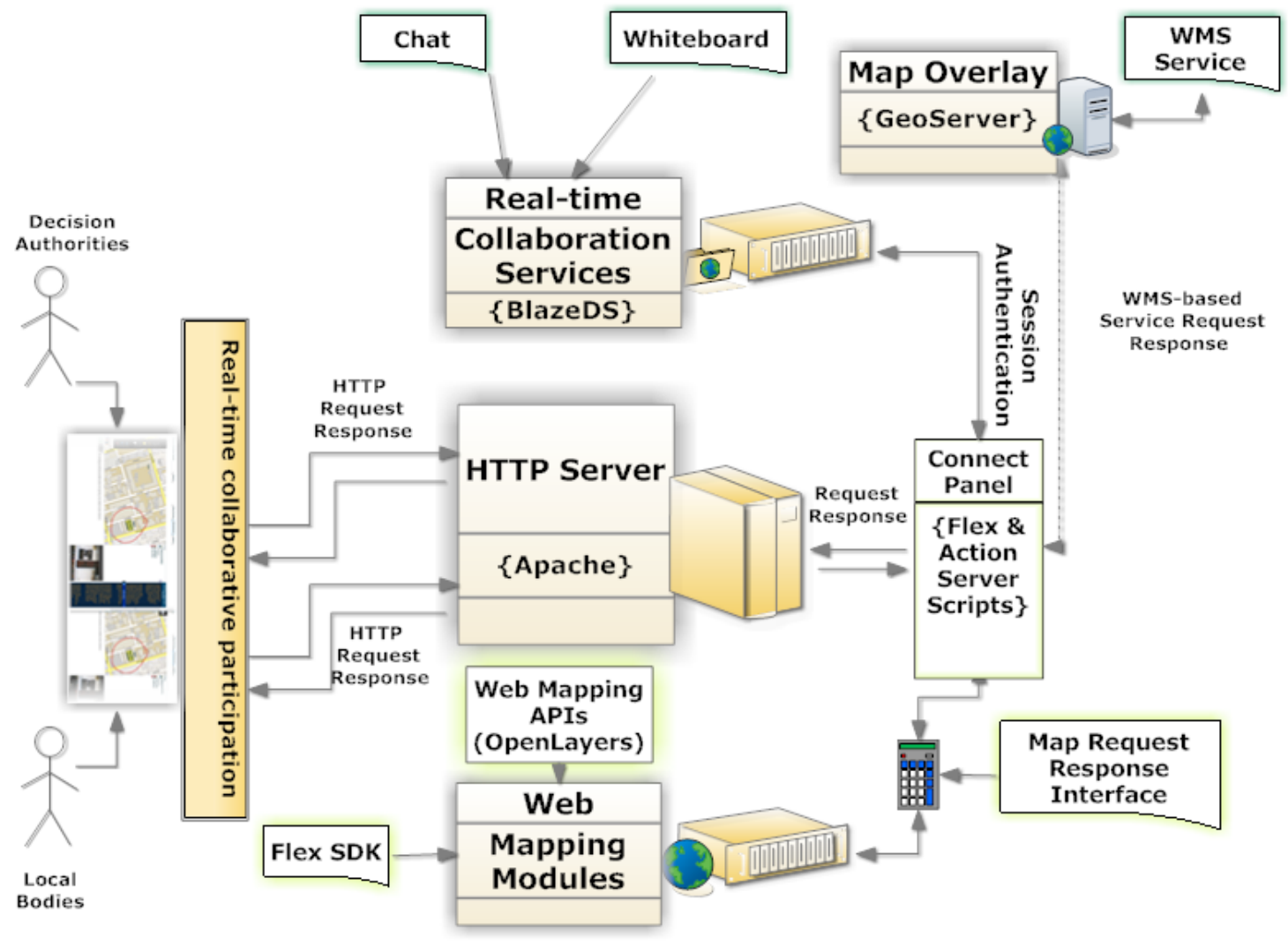

Figure 4.1: High-level Architecture of Co-PPGIS Synchronous Participatory Environment

CSPE uses Flex and JavaServer Pages (JSP) programming languages on the server side for real-time data sharing (e.g., map feature-based chatting, map-sharing, audio/video sharing, and drawing incidents/annotations on the map). Geospatial functionality is enabled by GeoServer (using PHP and JSP server-sided requests) for fetching map layers (WMS/WFS), whereas PostGIS/PostgreSQL stores and processes geo-spatial data. CSPE Web client is a Flash, Jquery, EXTJS/GeoEXT and Javascript-based container, which can be loaded and started in any kind of Hypertext Markup Language (HTML) and Flash plug-in supported browser. As Adobe Flash plug-in is available for all standard browsers (e.g., Internet Explorer, Firefox, Chrome), the user is advised to download and install on his/her computer the latest version of the Adobe Flash plug-in. 
The flash-based client and BlazeDS use Java remote and Web real-time messaging technology for communication, which allows the user to connect to back-end distributed data (PostgreSQL-PostGIS) and push data to Adobe Flexbased RIA. All clients' requests, for instance, access of BlazeDS real-time data messaging access of GeoServer/ MapServer-based WMS layers and producing base maps using OpenLayer API for map-sharing, can be transmitted on top of the HTTP, which is the common standard protocol used by HTTP servers for accessing HTML-based pages on the World-Wide Web.

\subsection{Co-PPGIS Asynchronous Participatory Environment (CAPE) High-level Architecture}

The Co-PPGIS Asynchronous Participatory Environment (CAPE) component mainly supports the asynchronous map-sharing approach for public participation.

An archetype of the CAPE GIS-enabled discussion forum is showed in Figure 4.2.

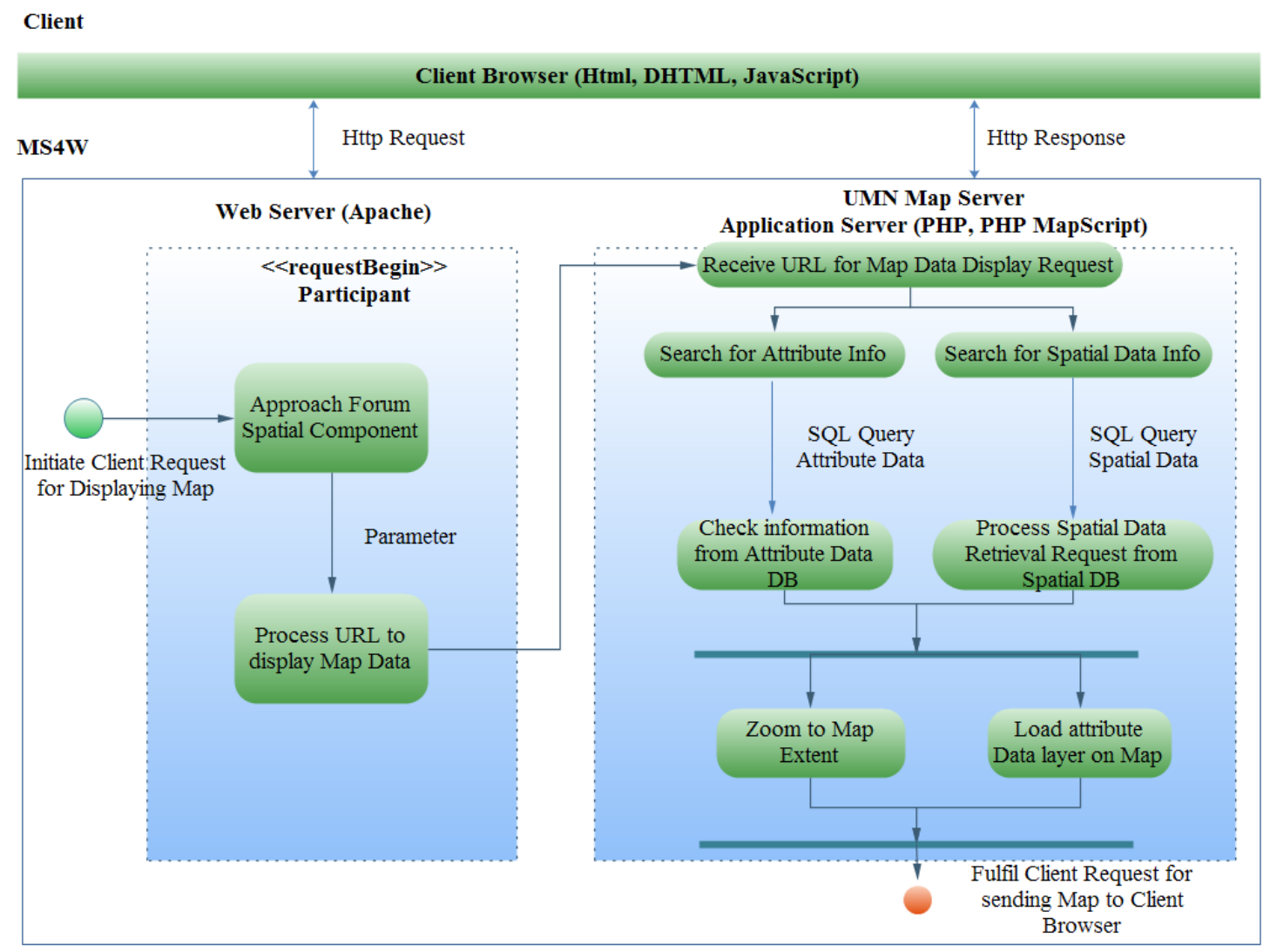

Figure 4.2: Logical Architecture of GIS-based Discussion Forum 
The organization of threaded comments in the CAPE component is similar to any discussion forum found on the Internet. The threaded forum messages are displayed in the main map panel. It initiates a concept of a GIS-enabled discussion forum in which public participants can enter and share ideas or information about spatially related aspects. In this component, a public participant can add, modify and/or view their own and other participants' comments. The GIS-based forum component is designed and developed using open source technologies. All of the forum textual and spatial data are stored in a PostgreSQL/ PostGIS database. This forum is embedded with another module, which handles all the GIS functionalities and can be initiated at any time during spatial context related discussions with other participants. All maps are produced using the University of Minnesota (UMN) MapServer.

\subsection{Co-PPGIS Component Service-oriented Architectures}

Figure 4.3 shows an overall flow between Co-PPGIS services, which are discussed in detail in this section.

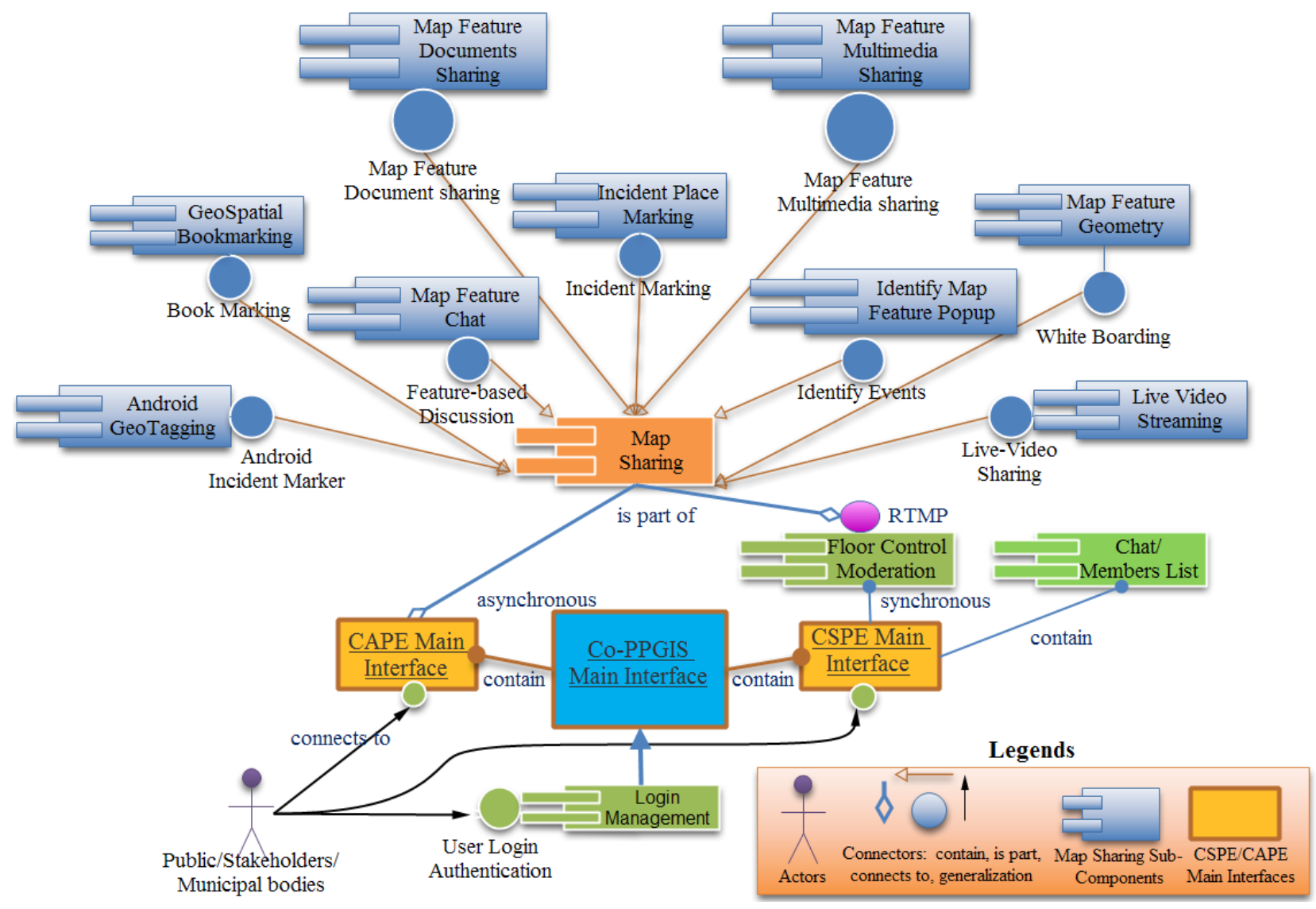

Figure 4.3: Flow Diagram of Co-PPGIS Component Services 
Figure 4.3 shows Co-PPGIS services and illustrates how stakeholders interact with each of its components. It depicts the components and service-oriented architectures of Co-PPGIS. CSPE and CAPE consist of several subcomponents and/or service modules (i.e., floor control moderation, android-based geo-tagging, live video streaming, member list and chat, annotate/draw map feature ${ }^{3}$, map feature-based chat, geo-spatial bookmark, place marker, identify map feature and map feature-based multimedia and document sharing). The central part (of the Figure 4.3) shows the Co-PPGIS main interface, which contains CSPE and CAPE components. As discussed earlier, CAPE was developed by adopting the multi-threaded forum-based concept. As shown in Figure 4.3, CSPE contains mapsharing and member list with a chat service. In addition, all components are combined and generalized ${ }^{4}$ into a mapsharing main interface. Moreover, a floor control moderation service, with the help and support of Real-time Messaging Protocols (RTMP) of BlazeDS and RED5, make available the real-time map and multimedia sharing features within CSPE. In addition, map-sharing mechanism services are developed to facilitate both synchronous and asynchronous participation approaches. The only difference in both mechanisms is that in synchronous mapsharing approach map and its related services are co-shared (having the same view) in real-time with other participants.

All of the component-based service modules are developed from scratch for the proof of concepts as a part of this study and are discussed in detail as follows:

\section{Login Management Service:}

There are two different ways to access the Co-PPGIS component services. For example, it can be set to direct or through login authentication depending upon the need and limited access of the municipal data to the general public. With direct access, the user can log in by selecting a screen name. In the login mechanism, only a registered user can $\log$ on in order to sharing data and services provided by the Co-PPGIS platform.

HTML Web pages are developed for user sign-in information, which initially is stored in the user profile database. The PHP has long been used with HTML forms to send parameters, such as name, email address, and receiving results. These HTML forms use the post method with server-side scripts to transfer user input parameters to the CoPPGIS application server. PHP scripts process these input parameters and pass them to the database for verification using the Structured Query Language (SQL).

\footnotetext{
${ }^{3}$ Map features can be categorized as objects, land, water, and other geographical features which can be represented using point, line and polygon and are important to the purpose of the map.

${ }^{4}$ Map-sharing generalization is the process in which different sub-components e.g., two or more sub-components services are combined into a generalized service interface. 
The user is then redirected to the Co-PPGIS's main interface from the login page if the result is verified from the database (Figure 4.4).

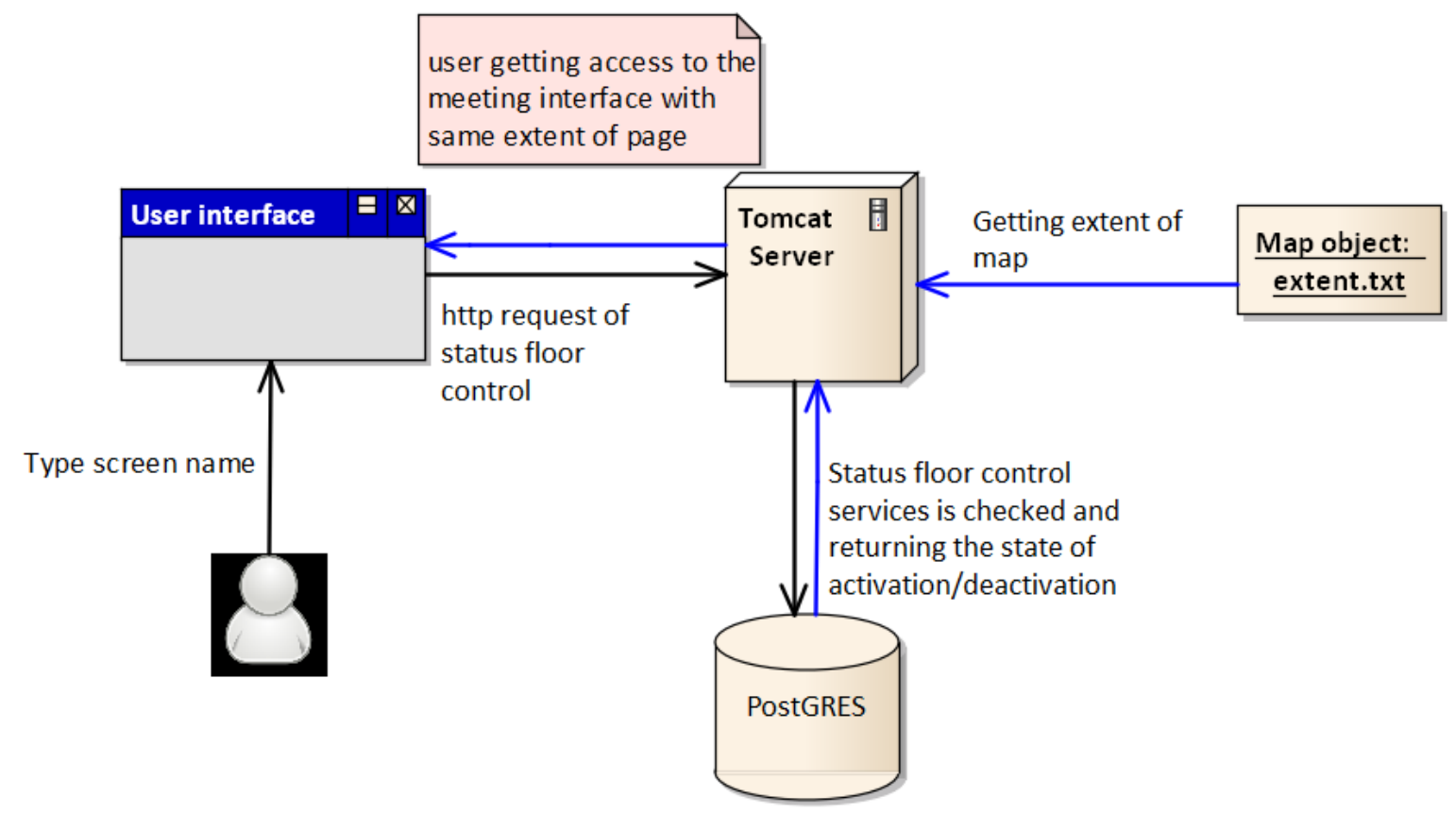

Figure 4.4: Information Flow Model of Login Management Service

\section{Floor Control Service:}

The floor controller processes the request when an administrator/moderator deactivates the main toolbars of the other attendees in order to stop a disturbance from other attendees during the Co-PPGIS collaborative meeting session. A message is sent to the BlazeDS server ${ }^{5}$ (through Flex server-side scripts function) and shared with other meeting attendees. Once the ID (unique key) of tool bar buttons is recorded and stored, users' map view and functionalities (i.e., use of toolbars, map panning and scrolling) will be deactivated. If any user wants to discuss anything or participate on the map interface, he/she must ask the permission from the moderator by sending a request through his/her application interface.

${ }^{5}$ BlazeDS is the server-based Java-remoting and Web messaging technology that support Real-time Messaging Protocols (RTMP) to push data in real-time to applications which are written in flex server-side scripting programming language. 
Figure 4.5 shows the information flow model of floor control service.

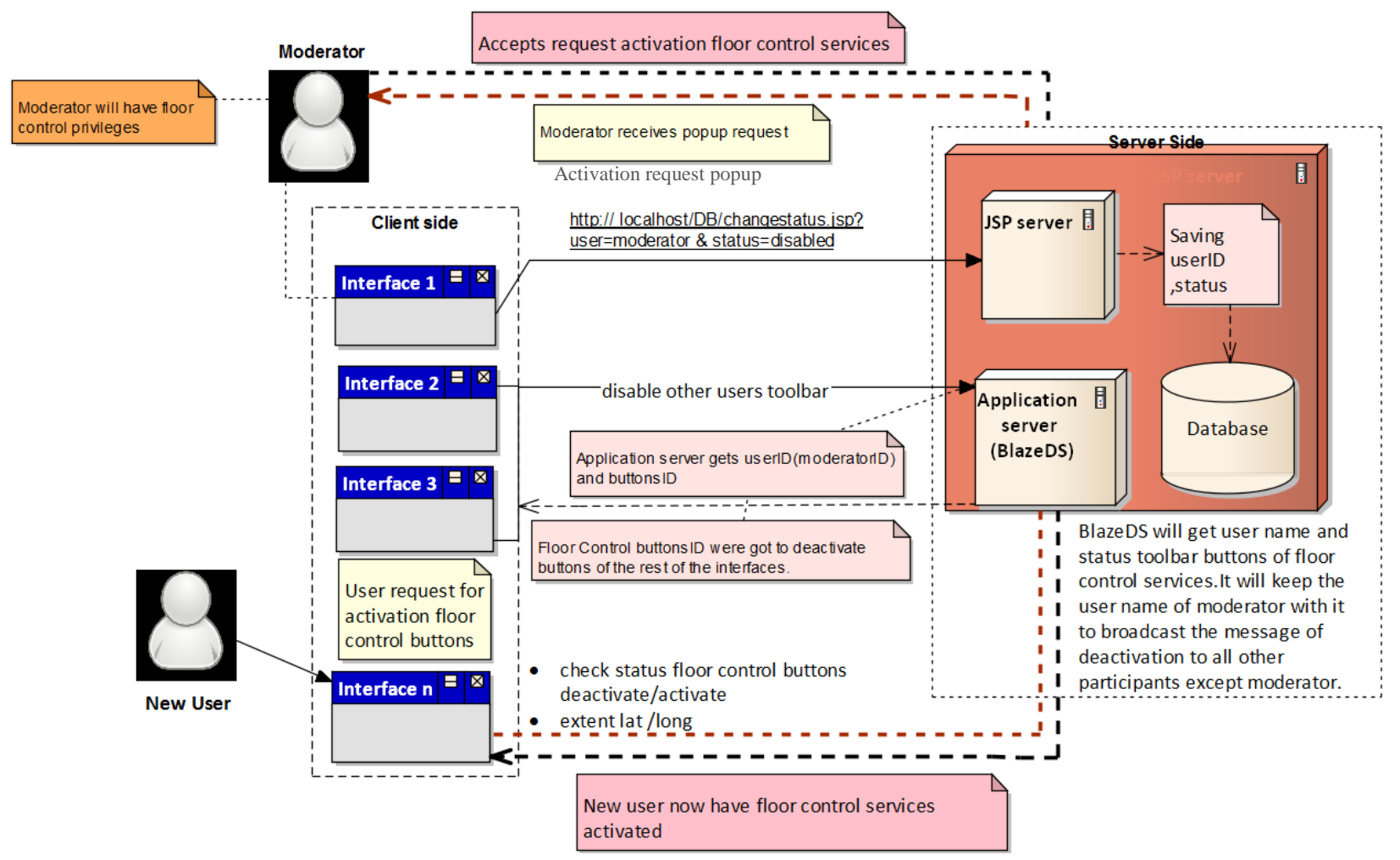

Figure 4.5: Information Flow Model of Floor Control Services

\section{Member List Service:}

When any user signs in, the flex controller saves the user name and pushes it into a user list panel that has been developed using Flex-based server-side scripting programming language. This widget is shared in HTML Division (DIV) element (DIV elements are the generic container that indicate a block of document content for flow and/or are used to define parts of a document) with other users using BlazeDS in order to synchronize and updates the users' names on the list panel. 
Figure 4.6 depicts the user list management service of Co-PPGIS map-sharing environment.

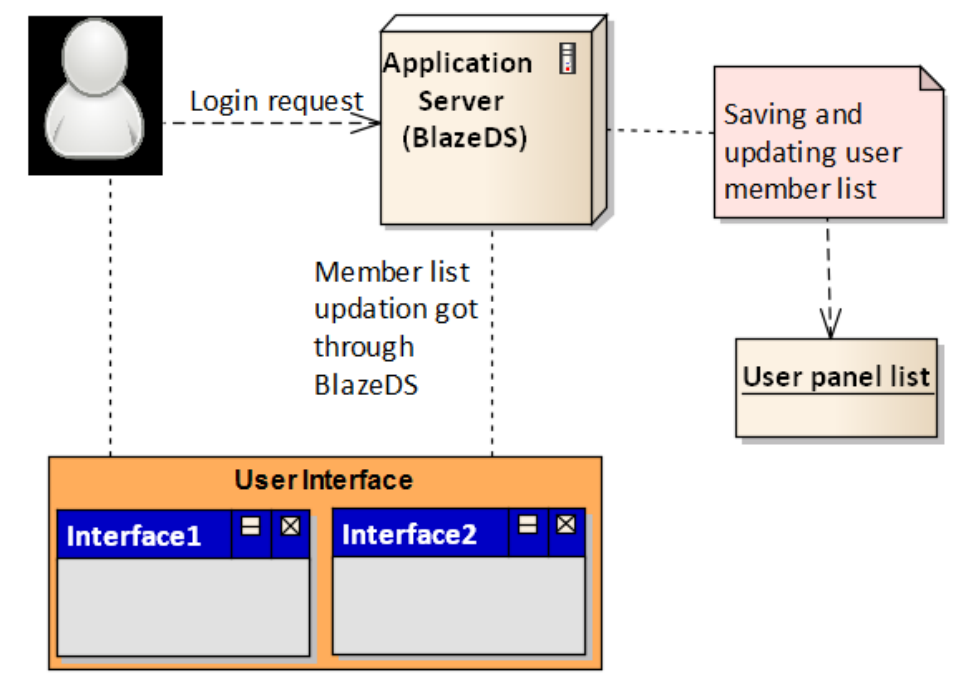

Figure 4.6: Users List Management Service

\section{Map-sharing Service:}

The map-sharing concept is developed and achieved using Flex and JavaScript programming languages. For example, in the map-sharing rendering environment, when a user changes the zoom or extent of the map, the value of set function is accessed or recorded using the JavaScript function and passed on to the flex server (BlazeDS), which in turn processes and responds to all users for map synchronization. As a result, every user shares the same map area (extent) on their interfaces (see Figure 4.7).

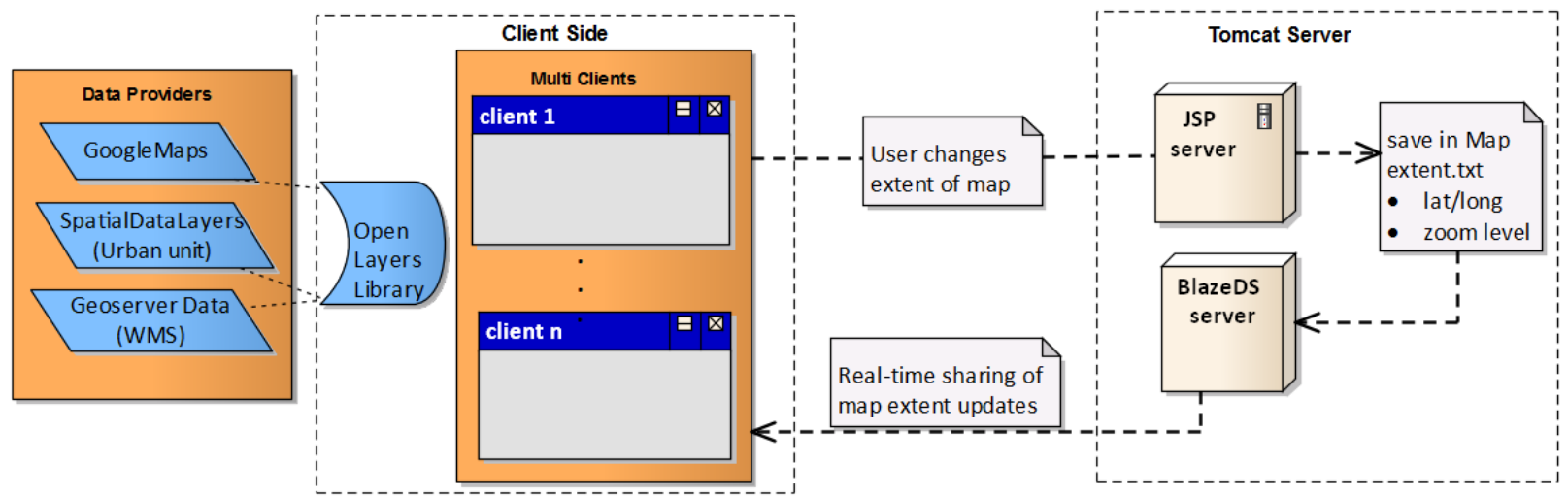

Figure 4.7: Information Flow Model of Map-sharing Service 


\section{Android-based Geo-tagging Service:}

An Android-based service provides a way to annotate/geo-tag areas of interest related to municipal planning and development from the field to capture real-time data (i.e., on-going issues and progress of a municipal planning project). Users can share and discuss their points of view and provide their input anywhere at any time using this service. For example, the user captures and updates the project related information through a Java-based application installed on Android-based devices. Textual information and multimedia data are converted to (Extensible Markup Language) XML format and stored in the database through Java Server pages (JSP) and XML writer. The XML parser reads the XML data and the information is displayed on the map using JavaScript programming language. Figure 4.8 depicts information flow model of android-based geo-tagging service.

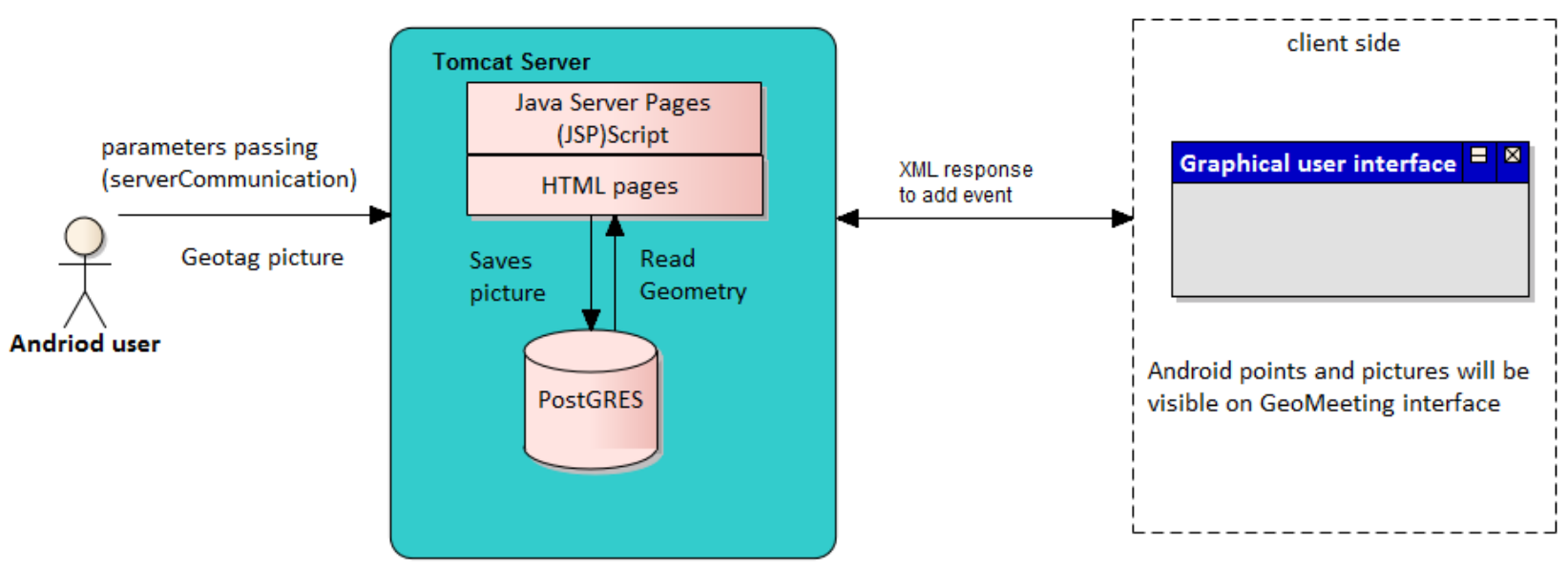

Figure 4.8: Android-based Geo-Tagging of Incidents on a Shared View of Map

\section{Chat Service:}

The chat object is developed using flex and action script programming languages. When a user types some text in the chat window, it is passed on to the flex server-side scripts and is shared among users using the BlazeDS realtime messaging service. 
Figure 4.9 depicts the general chat service of Co-PPGIS map-sharing environment.

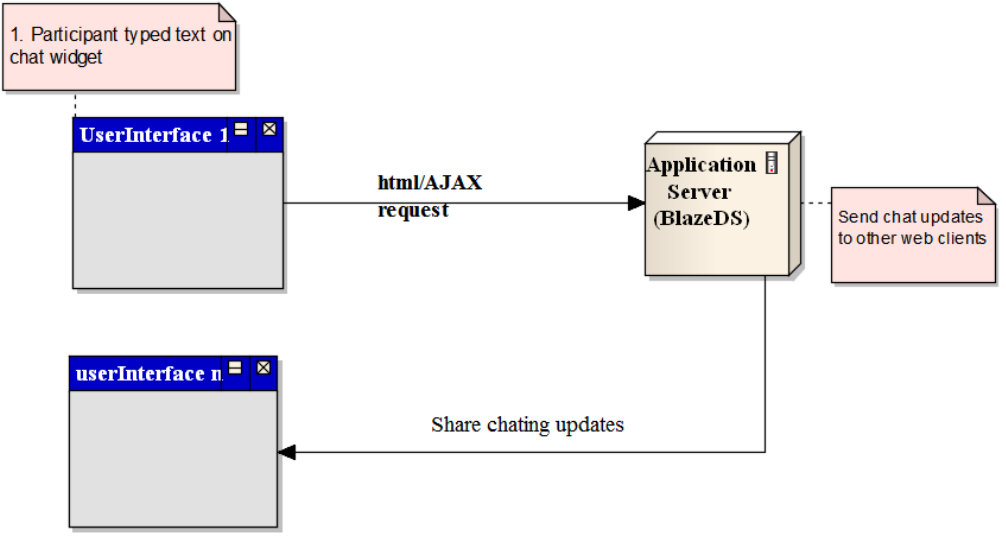

Figure 4.9: General Chat Service using BlazeDS and Flex

\section{Map Feature-based Chat Service:}

The map feature-based chat component is written in JSP and jQuery (a simplified form of JavaScript programming language). The pop-up window process controller receives the information entered by the user and passes it to the JSP server using a jQuery Get method. Later, JSP provides this information to the database through an SQL for the storage and retrieval of the message posted. Chat messages are shared in real-time among different meeting attendees using BlazeDS Server (Figure 4.10).

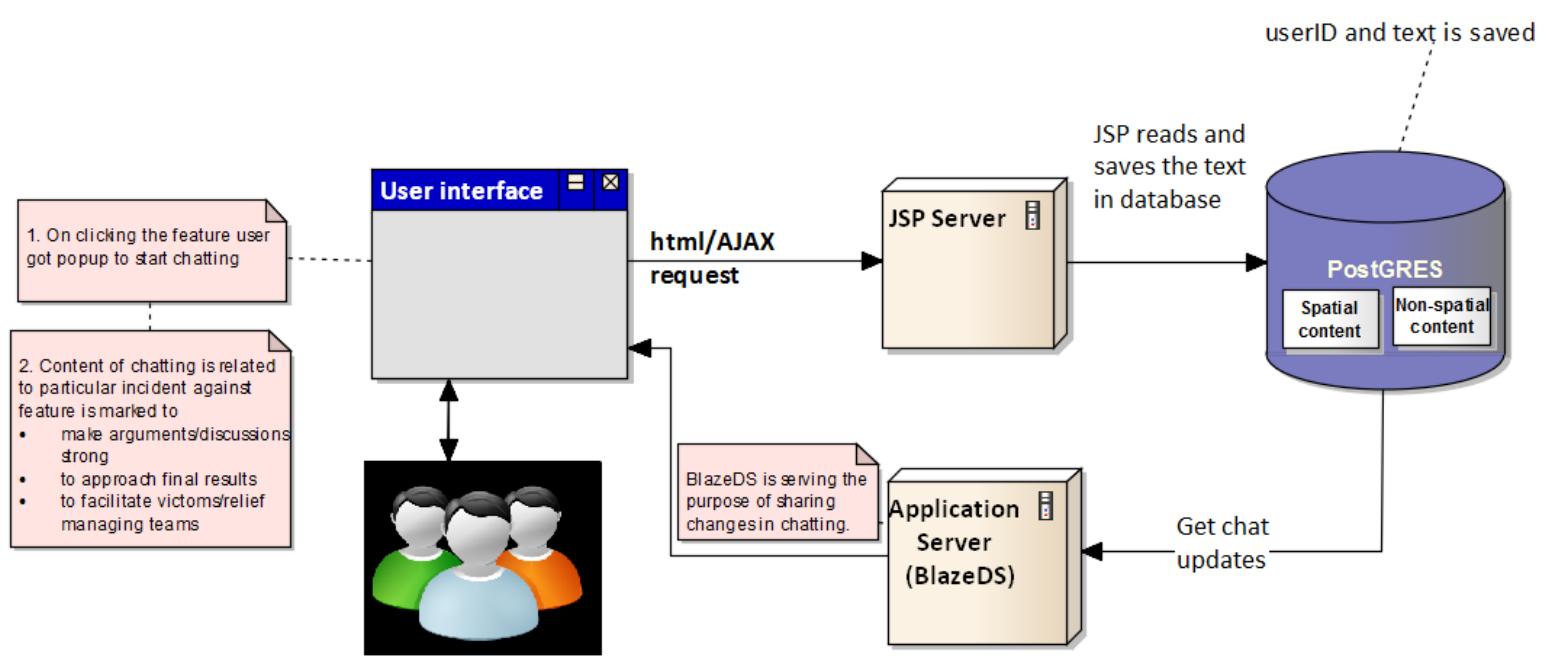

Figure 4.10: Map Feature-based Chatting against Marked Incidents on a Shared View of Map 


\section{Map Feature Identifving Popup Service:}

The identifying map feature pop-up is developed for identifying the selected feature properties. Upon feature selection, a JavaScript event calls for identifying and displaying all the features' attributes inside the pop-up window using HTML (attributes are displayed against each feature when the data of each layer is parsed using XML from the database).

Figure 4.11 shows information model of identify feature popup service.

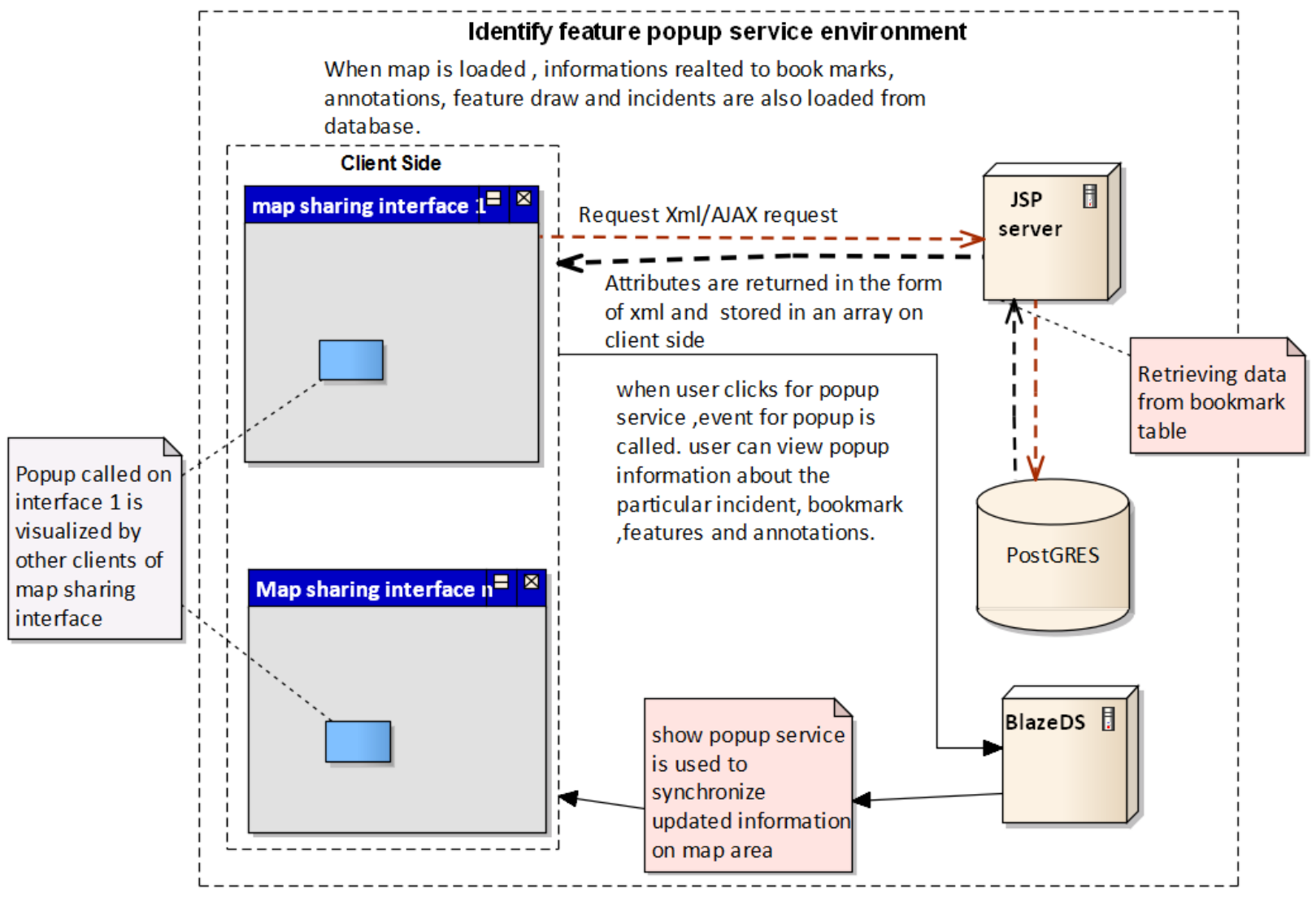

Figure 4.11: Identify Features Popup Service

\section{Geometry Map Feature Service:}

The geometry map feature service is written in JSP and JavaScript languages. When a graphical feature is drawn (such as point, line, polygon, circle, annotation, and hexagon) on a map, the geometry object of the feature is accessed or inherited through an open layer JavaScript library. If this library is saved on the web-server, then it can 
be read by client browsers. Otherwise, it can be accessed via the online URL. On the server side, a JSP written program can then read and parse (through Get method) the text and save geometry into a PostGIS (PostgreSQL) database. In response, a JSP component reads results (i.e., geometry and attribute's information) from the database using SQL and sends converted XML to a JavaScript program for parsing and displaying map shapes (Figure 4.12).

Another way of creating a basic GIS geometry function (i.e., point, line and polygon) is using Web Map Service (WMS), which is a standard protocol for serving geo-referenced map images over the Internet served through GeoServer (an open source map server for generating maps). The stored geometry and attributes are published to GeoServer and can be accessed or read in browsers through JavaScript and open layer functions. Every time, a new feature is created on a map, the JavaScript function takes the ID of each drawn feature as an event and passes it to the BlazeDS, which shares the feature among all the participants in the session.

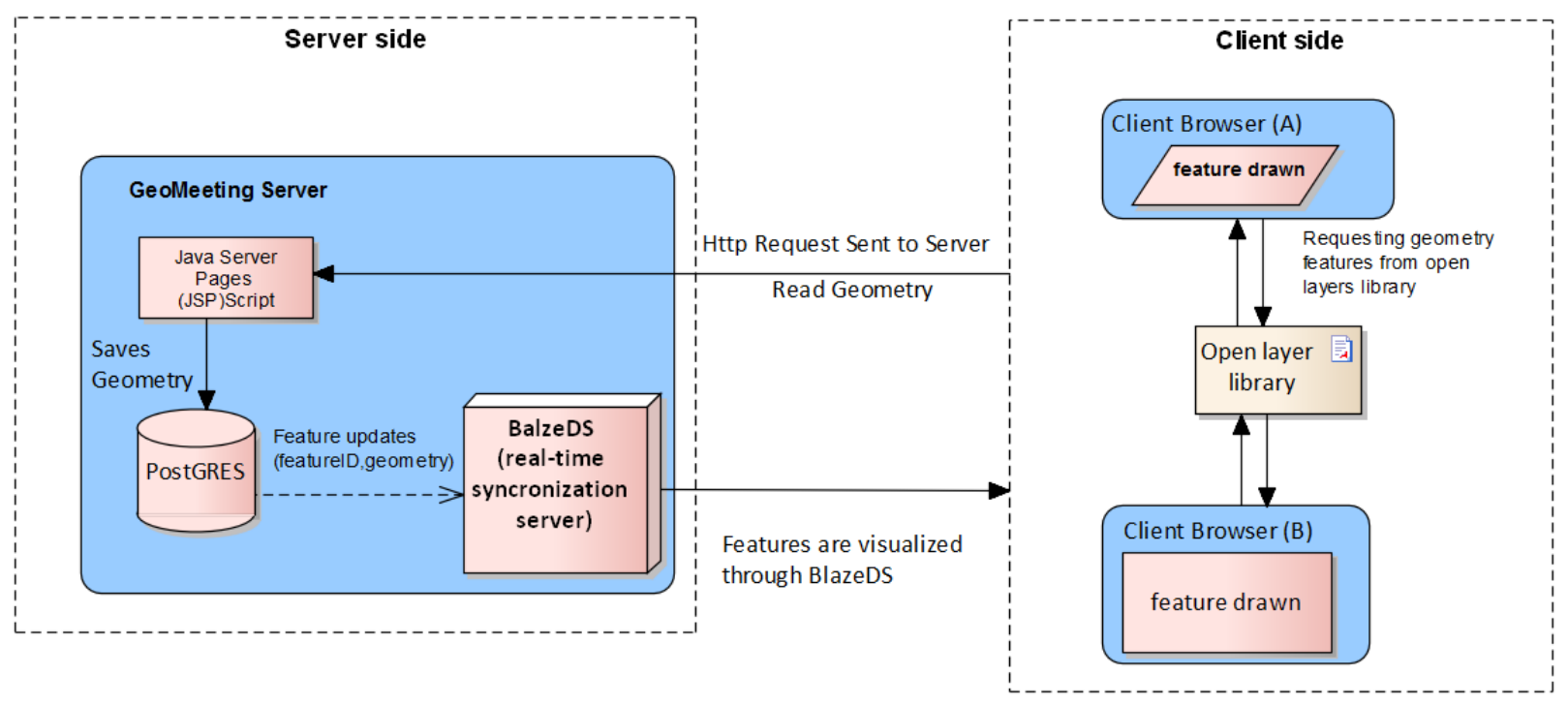

Figure 4.12: Geometry Shapes Geo-tagging of Incidents on a Shared View of Map

Some other basic GIS functions that are provided by this service include measure, identify, pan, zoom in, zoom out, zoom previous, forward, full extent, search, zoom, select by bounding box, and buffer.

\section{Map Feature Multimedia Sharing Service:}

Map feature multimedia sharing service is developed to attach multimedia objects (i.e., images, audio and video files) to each incident or geometric shape in order to share rich information among attendees. All multimedia files 
are saved directly on the web-server, whereas feature information and multimedia objects are retrieved using JSP. Multimedia is categorized based on its type, and each category is displayed in different GeoEXT (accordion) panels (Figure 4.13).

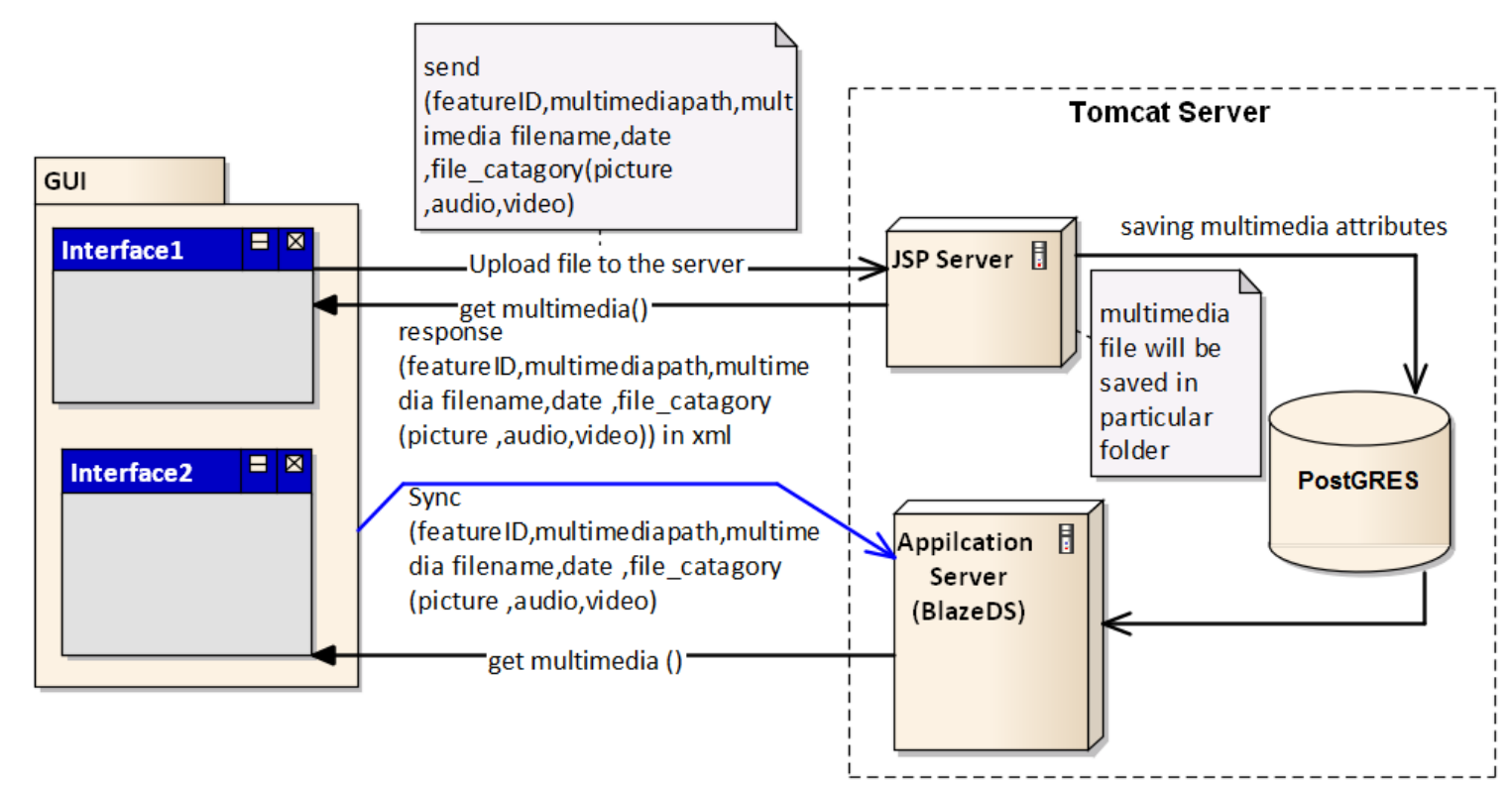

Figure 4.13: Multimedia Geo-tagging Related to Incidents during Map-sharing Environment

\section{Geo-Spatial Bookmark Service:}

The bookmark service is created to save the extent of the map where an important discussion was made by different participants. When a user clicks on the bookmark, the extent and zoom of the map are recorded or stored in the database using the JSP Get function, which can be shared in order to allow the users to understand other participants' contributions. 
Figure 4.14 shows the geo-spatial bookmark service of Co-PPGIS map-sharing environment.

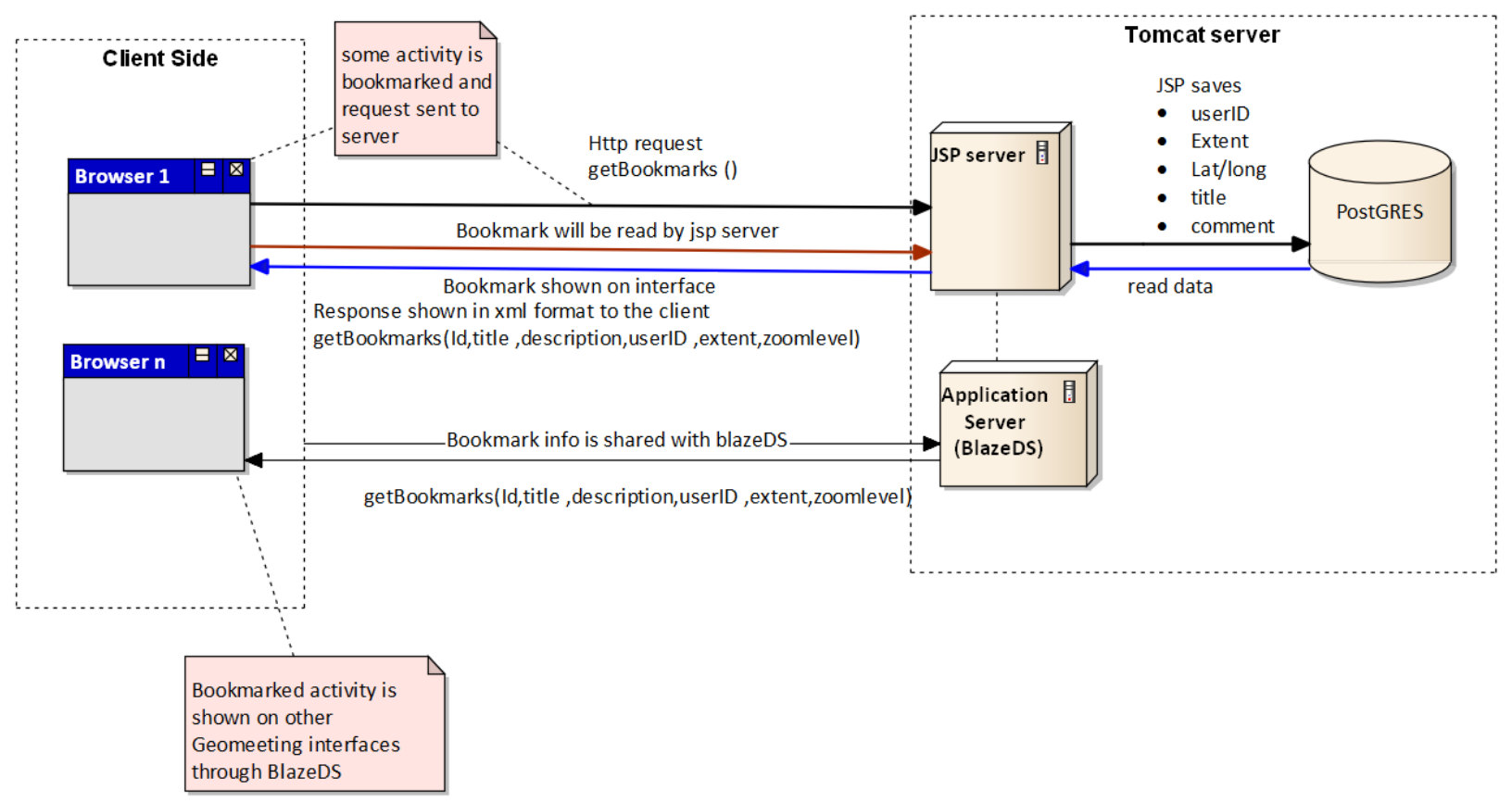

Figure 4.14: Bookmark Service for Saving Map Extent with Annotation

\section{Editing Toolbar Service:}

The Co-PPGIS prototype supports a very simple OpenLayer API-based editing toolbar, which can be initiated from the map interface to make geo-referenced annotation, such as point, line and polygon, to represent any real-world entities during a collaborative discussion. Geometrical annotations are stored into a PostGIS database. Annotation layers are then displayed and rendered with custom symbology to the meeting interface using GeoServer. Annotations are co-shared among participants using BlazeDS real-time data-sharing protocols. The developed mechanism of geo-reference based marking is so simple that any participant can easily focus on the meeting discussion while simultaneously making annotations. 
Figure 4.15 depicts the editing toolbar service of Co-PPGIS map-sharing environment.

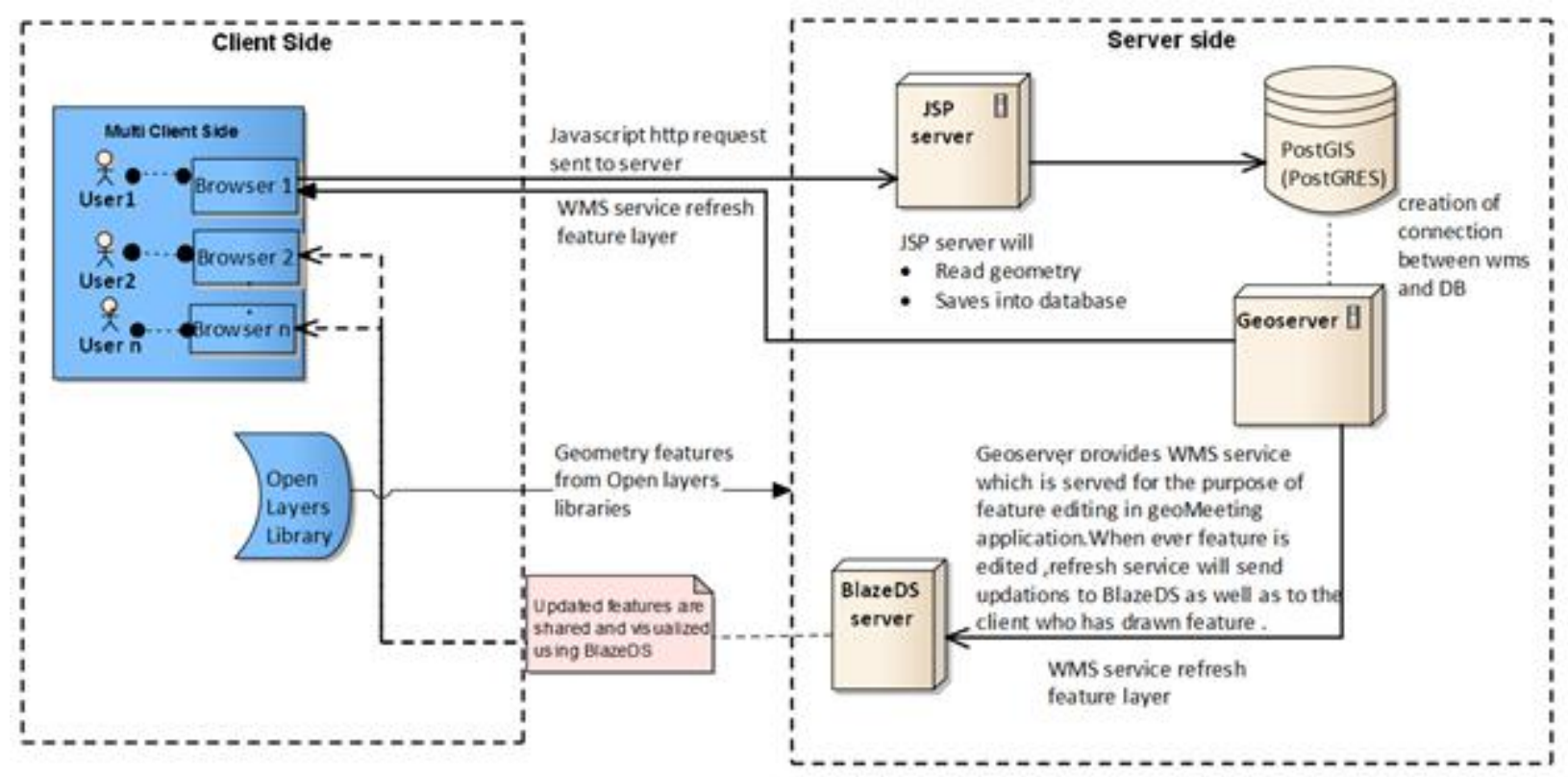

Figure 4.15: Editing Toolbar Service during Spatial Discussion for Drawing Annotation

\section{Live Video Service:}

With the live video-based interactive communication service, any person can share audio and/or video while inputting his/her ideas on a map. The Co-PPGIS prototype video service is developed using Flex, Action Script and JAVA programming languages. The Co-PPGIS Web client accesses the RTMP protocol of the RED5 streaming video server in order to share the video among participants. A GeoExt-based video pop-up supports a very simple and user-friendly interface so that everyone can focus on the Geo-collaborative discussion. The participants do not have to install anything (even to broadcast audio - a single click starts video conferencing between multiple participants. For more system user's behavioural understanding in terms of user interaction with the real-time services of the Co-PPGIS, see Appendix 6, which depicts all related UML-based sequence diagrams in connection to the above-explained services-oriented architectures.

\subsection{Prototype Implementation}

The initial prototype is developed to demonstrate some aspects that help to improve the participation process (i.e., awareness, information access, participation, and feedback). The following sections describe the implementation of 
asynchronous and synchronous participation approaches to the Co-PPGIS prototype, which is based on the services discussed in the previous section.

\subsubsection{Co-PPGIS Asynchronous Participatory Environment Blog Component}

The CAPE blog interface is developed based on an asynchronous participatory approach to provide GIS-based discussion forum support. The interface is designed for facilitating public and/or stakeholders to meet at common virtual place and record their input in relation to municipal planning-related discussions. It supports collaborative participation during the planning and decision-making process workflows. The prototype's blog interface with York Regional Municipality amendment plans information (the Google map and municipality vector data) is shown in Figure 4.16.

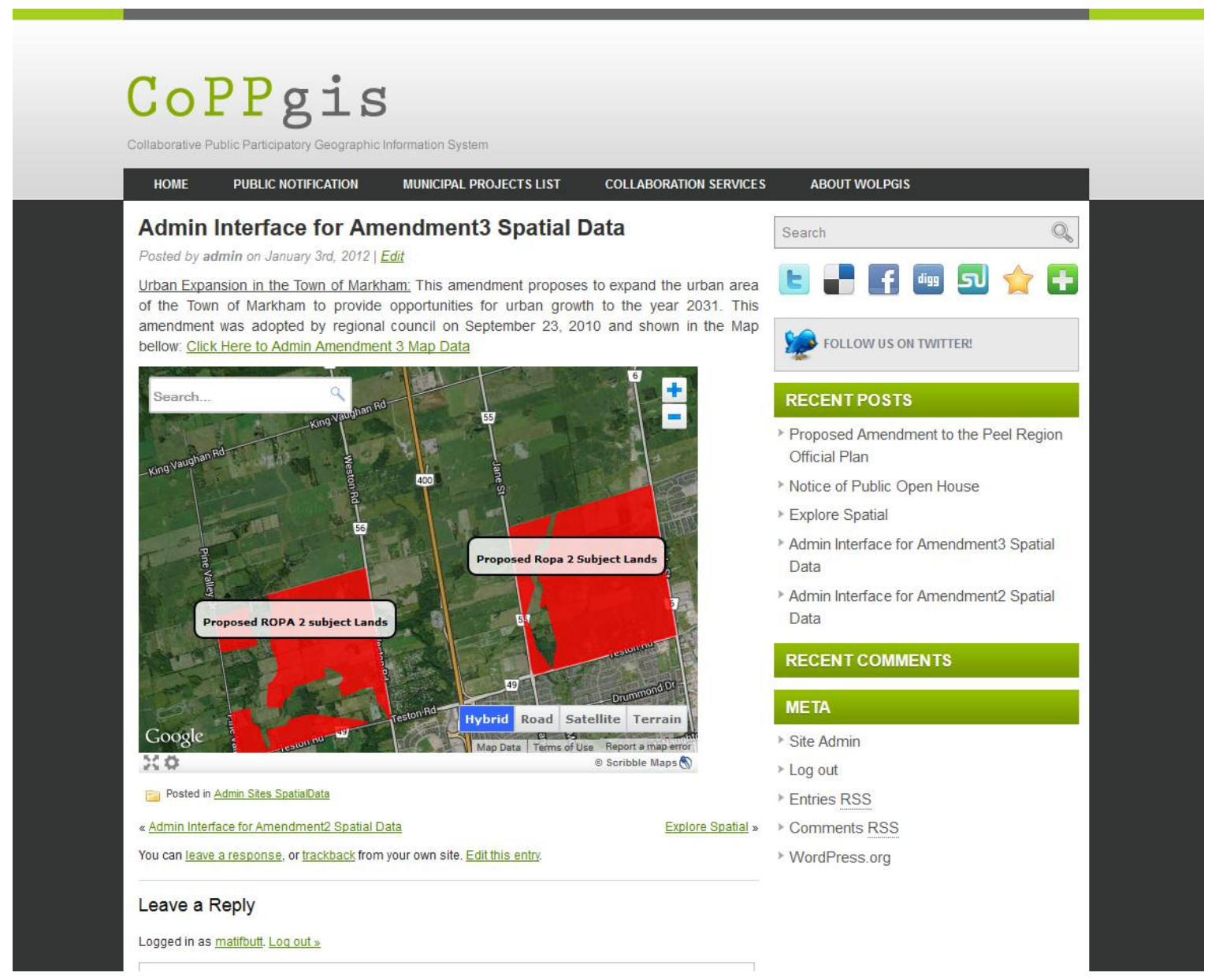

Figure 4.16: Spatial Data of York Region (Town Markham Amendment Plan) is Overlaid on the Google Map (can be accessed at http://coppgis.com/wolpgis-f/?p=54) 
This information is taken from the York Region's official site (http://york.ca) to create a mock-up test scenario. The usability evaluation process of the CAPE blog's spatial component is described in detail in Chapter 5. The purpose of this usability test is to evaluate how well the prototype can help facilitate the participants by providing a userfriendly interface.

Participants in the discussion are notified and invited to join the discussion in order to identify planning concerns with the urban expansion in the town of Markham. Spatial data of Amendment 3 can be annotated for collaborative discussion. Moderator/admin can contribute (Add, modify, delete features, etc.) on the map and other participants can view and submit their feedback collaboratively in order to improve this plan. Each participant can initiate discussion within the blog posts with which he/she is mostly concerned. This component provides participants with the ability to contribute and/or become involved in the planning and development process workflows. In addition, Web objects, including blogs, photos, videos, project information and notifications, are embedded into a map by the APIs of Google and scribble map service providers, where textual messages are associated with geographic information.

\section{- Municipal Project Awareness}

One of the requirements of Co-PPGIS is to manage multiple new or ongoing projects of municipal projects that involve high levels of public participation. Therefore, a common interface is designed for all projects to share the same characteristics, which requires the same set of functions to support collection of public inputs and collaboration. The interface of any project can be accessed through a project list. The project list also acts as a navigation tool to allow participants to switch between different project interfaces easily.

\section{- Information Access}

Access to project information enables participants to build a necessary understanding of the ongoing and future project under consultation, and is vital to successful participation, as the Web has proven an excellent platform for information dissemination. The Co-PPGIS prototype blog components manage documents, meeting agendas and minutes, bylaws and regulation documents, and the contact information about each project in one place. The public can easily browse and view the information organized here and if appropriate, replay to public meeting videos or previous collaborative sessions. The contact information includes the name, email, phone, and fax numbers of the moderators or authorities/stakeholders. 


\section{- Notification: Public Notice System}

The online public notice system plays two important roles: (a) notifying the registered participants of upcoming public meetings/events using a newsletter function and SMS, and allowing the interested citizens to explore projects' information and get prepared for the public meeting, and (b) providing a platform for continuous soliciting of public input and presentation of results. The notification component also provides a location mapping function in which a participant can find his/her way or direction to the public meeting place. For this direction service, open source APIs from Google Maps, with a mash-up of a PHP server-side scripting language is used.

\section{- Participation: GIS-enabled Discussion Forum}

In addition, the foremost spatial component of the prototype was implemented with basic GIS functions (i.e., zoom, pan, save map extent, load previous map extent) to explore planning and development scenarios within the geographical contexts of the projects. This component was especially designed for those stakeholders who are technically well-informed. This component allows them to examine alternatives at their own pace. For example, they can explore "what-if" scenarios using the simple Web-based GIS component. Figure 4.17 depicts the CoPPGIS GIS-based discussion forum component.

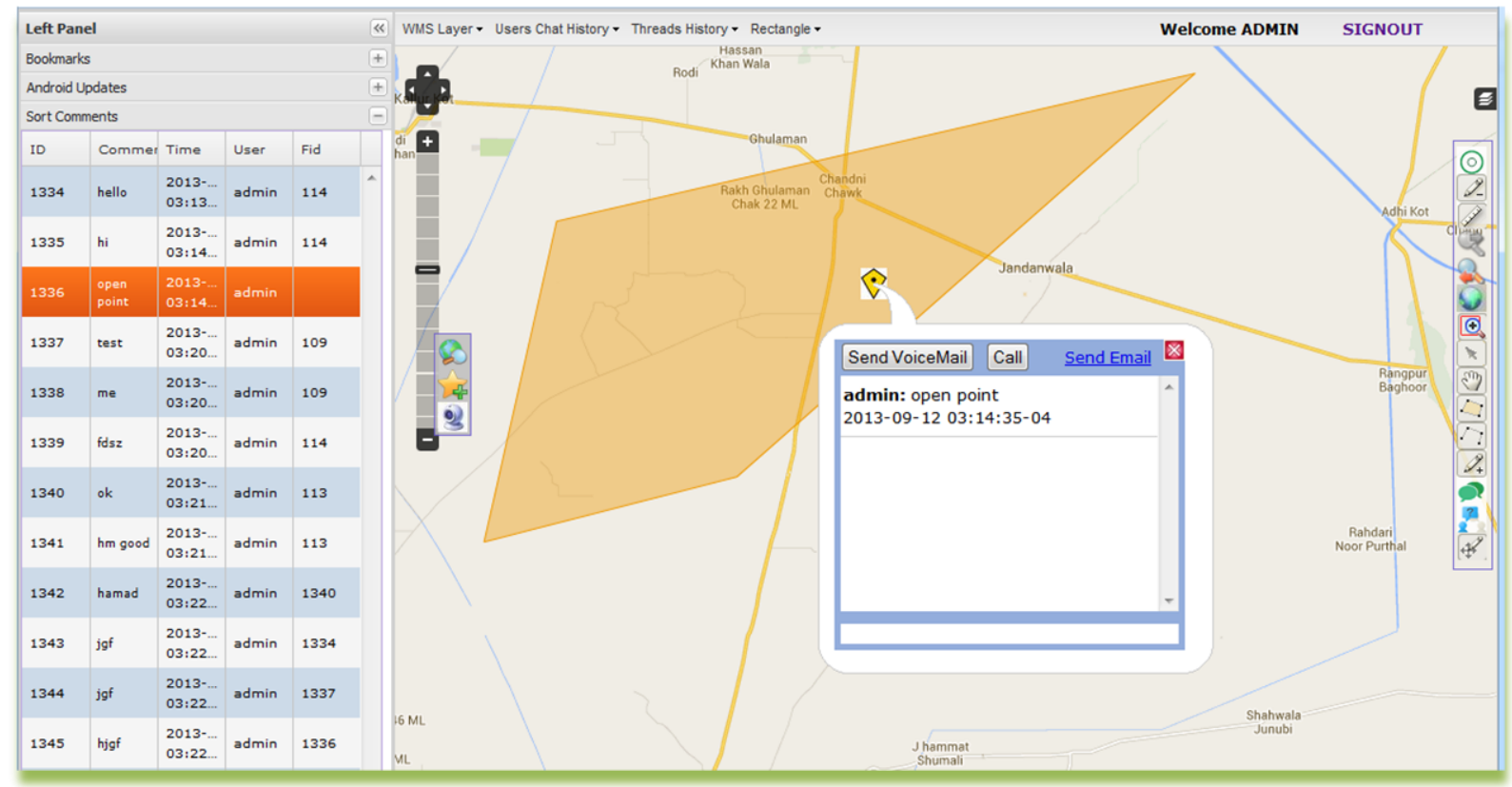

Figure 4.17: Co-PPGIS GIS-based Discussion Forum Component (Online access URL: http://coppgis.com/prism) 
The spatial component was further enhanced to include more functions required by municipal planning. In the component, spatial information was provided in the form of a vector data layer. Different tools, (e.g., zoom in, zoom out, whiteboard tools with their specific functionalities) were provided in the toolbar whereas, layer's information and results are displayed in the layer panel of the prototype's interface. To incorporate the general public, the spatial component was modified into a GIS-based discussion forum. One of the key characteristics of GIS-enabled or augmented discussion forums is to link threaded postings to a geo-reference map or the comments/documents posted by stakeholders. The GIS-based forum component has been developed using open source technologies such as EXTJS, GeoEXT, and OpenLayer Spatial Templates. Its organization of threaded postings is similar to any discussion forum found on the Internet.

The latest version of the Co-PPGIS asynchronous map-sharing component is showed in the Figure $4.17^{6}$. The forum allows participants to share and input their ideas anywhere/anytime using a typical web-based discussion forum, but with the extended capability to invoke a Spatial Data Handler to annotate on the map. The Spatial Data Handler is developed to import spatial data as layers from local and remote computers. Bookmark functionality enables the participants to save or load map extent with its past threaded discussion. A user can store the important documents related to municipal project, along with the map features, attach saved video/audio session to his/ her postings in the discussion forum and share with others. Other participants can view or edit the saved map information and project document, and then, repost it.

\subsubsection{Co-PPGIS Synchronous Participatory Environment (CSPE)}

The Co-PPGIS Synchronous Participatory Environment (CSPE) is a web-based geospatially-enabled conferencing system that integrates real-time and synchronous map-sharing, chatting, geo-referenced map annotation, user and meeting management for supporting discussions among multiple users geographically located at different places for consensus building on a problem. The system supports real-time integration of data from different sources through web mapping service APIs, and encourages the integration of local knowledge expressed by meeting participants.

Built based upon the conceptual frameworks of prior research prototypes, which primarily used proprietary technologies (i.e., Adobe Life Cycle Data Services, Adobe Cocomo, and Media server), this new version focuses on the redesign and re-development of the system using open source technologies as well as web map services based on

\footnotetext{
${ }^{6}$ Latest versions of spatial component can be accessed using username $=$ test, password $=$ test at: http://coppgis.com/prism, http://coppgis.com/cpgis, http://coppgis.com/cppgis, http://coppgis.com/ppgis, http://coppgis.com/pgis

${ }^{7}$ Previously designed spatial components can be accessed at: http://coppgis.com/wolpgisGSC_FF/wolpgisGSC_FFMain.php?site=example_bc_win http://coppgis.com/wolpgisgsc/map.phtml
} 
OGC standards and other open standards. CSPE is developed from scratch, integrating OpenLayer and Adobe flex technologies and following interactive incremental development approaches. Figure 4.18 depicts the implementation of CSPE, which shows the important sub components, (i.e., real-time project document sharing and map-sharing) related to the CSPE developed during this research study.

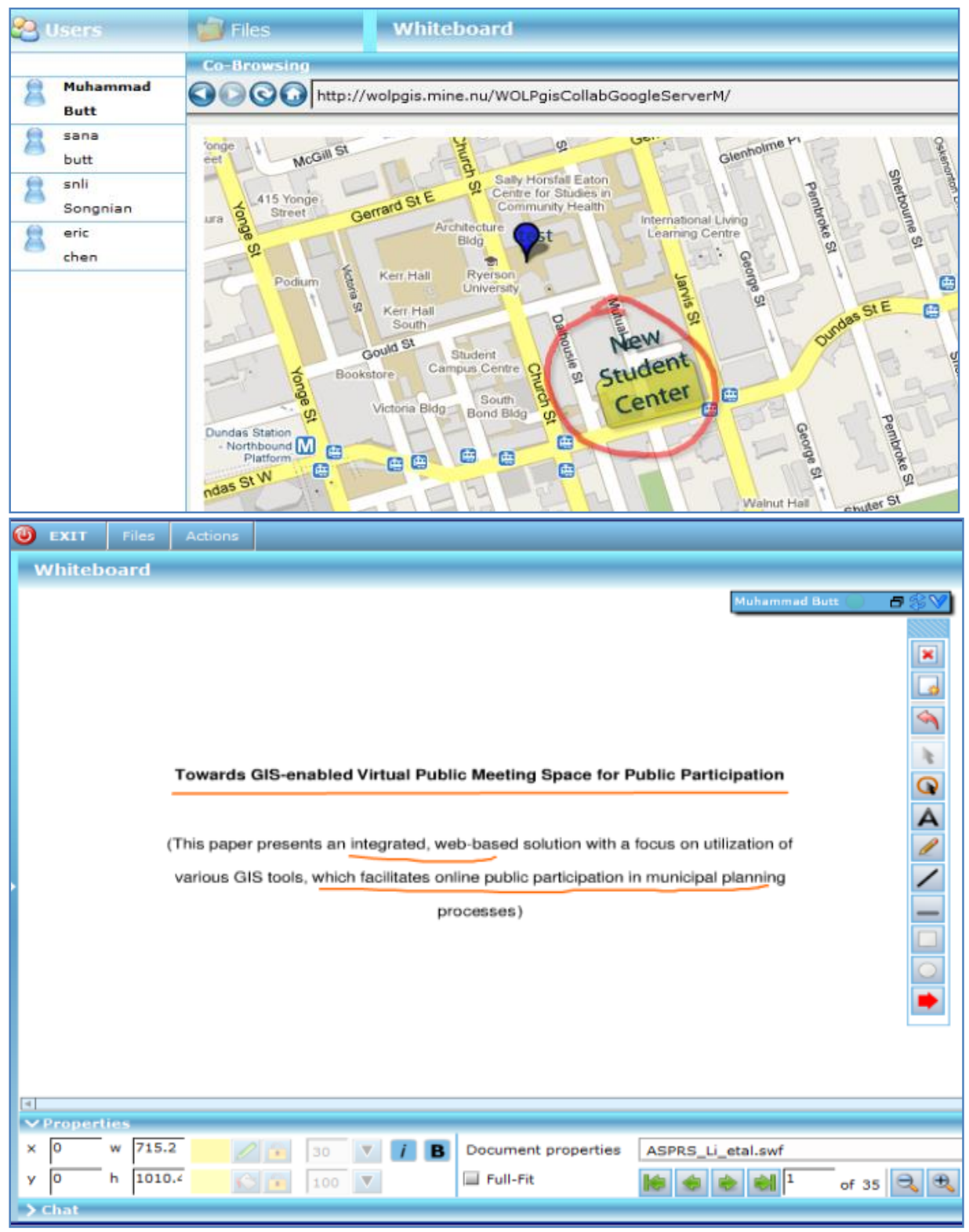

Figure 4.18: CSPE Interface with Real Public Meeting Document Sharing and Basic Map-sharing Streams (Online access URL: http://coppgis.com:8080) 
In Figure 4.18, the simple map-sharing component is shown, which has a real-time map-sharing feature with some basic whiteboard annotation tools. A picture is worth a thousand words. For many municipal planning-related discussions, a map or map-based display of different scenarios shared by participants greatly facilitates the discussion on some issues. For this reason, a CSPE simple map-sharing component was developed to allow participants to collaboratively explore the geographic context of the projects while discussing some issues. The advanced version of the CSPE collaborative map-sharing component is discussed in detail with its major functionalities walkthrough in next section.

Furthermore, this $\mathrm{CSPE}^{8}$ integrates a number of features, including simple map-sharing, chatting, audio, video conferencing and white boarding, into a single interface that can be invoked and run in a new window. It is intended to be used by a group of stakeholders who have a common topic, but different views on that topic. Access to the map helps them to make their points clear. Any stakeholder can initiate a collaboration session, invite others to join and give their valuable suggestions. All participants who join the session have the right to use whiteboard tools.

This CSPE is a supplement to real public meetings for those who cannot physically attend the meetings. The idea is to stream real public meetings online and provide facilitating tools to enable online participants to question and interact with each other, as well as to allow presenters to integrate their electronic presentations and maps into the meeting environment (see Figure 4.18).

\subsubsection{Co-PPGIS: Collaborative Map-sharing Component}

By incorporating the enhanced real-time map-sharing component into CSPE, the prototype interface allows the presenters to share map displays in the same way as PowerPoint slides are shared on screens in many other web conferencing systems. The presenters can also use the built-in whiteboard tools to select features, add annotations, and draw graphics. During the virtual meeting session anyone can be the presenter; however, only the presenter can initiate the tools and control how the map data should be displayed to facilitate their presentations. Co-PPGIS mapsharing functionality and interfaces were revised and improved periodically. The main idea was to provide usable interfaces to the public for their involvement during municipal planning and management related activities 9 . Moreover, some old functions were rectified, and some new features were added to make it more effective and

${ }^{8}$ Co-PPGIS Synchronous Participatory Environment can be accessed at: http://coppgis.com/WOLPgis-GeoMeetSocial/blogs/ http://coppgis.com/WOLPgis-GeoWebEx1/?page id=52, http://coppgis.com/WOLPgis-R1/

${ }^{9}$ Direct access to different versions of Synchronous Map-sharing Components: http://coppgis.com/geomeet1, http://coppgis.com/geomeet4, and http://coppgis.com/geomeet5 (these versions have some basic map-sharing functionalities and used during usability testing of the Co-PPGIS synchronous map-sharing mechanism in the Chapter 5) 
interactive. For instance, the participants can find the tooltip text by hovering over a mouse pointer on any tool's icon in order to make the map more balanced on different monitor sizes. The Co-PPGIS collaborative map-sharing component was developed from scratch; and hence, gradually improved to its advanced functionalities, which incorporates sharing of accessories of web meeting (i.e. sharing of documents, audio, video, picture, presentation).

Users may access directly the log-on page for the Co-PPGIS map-sharing component using a standard Web browser such as Mozilla Firefox 13+. Once the log-on page is shown, the user enters a participant name and joins the CoPPGIS map-sharing environment. After the meeting session is established, the Co-PPGIS map-sharing component will load its default interface. It may take time to load because web services are loaded from different sources depending on the Internet connection speed. The Co-PPGIS map-sharing component interface is shown in Figure 4.19 .

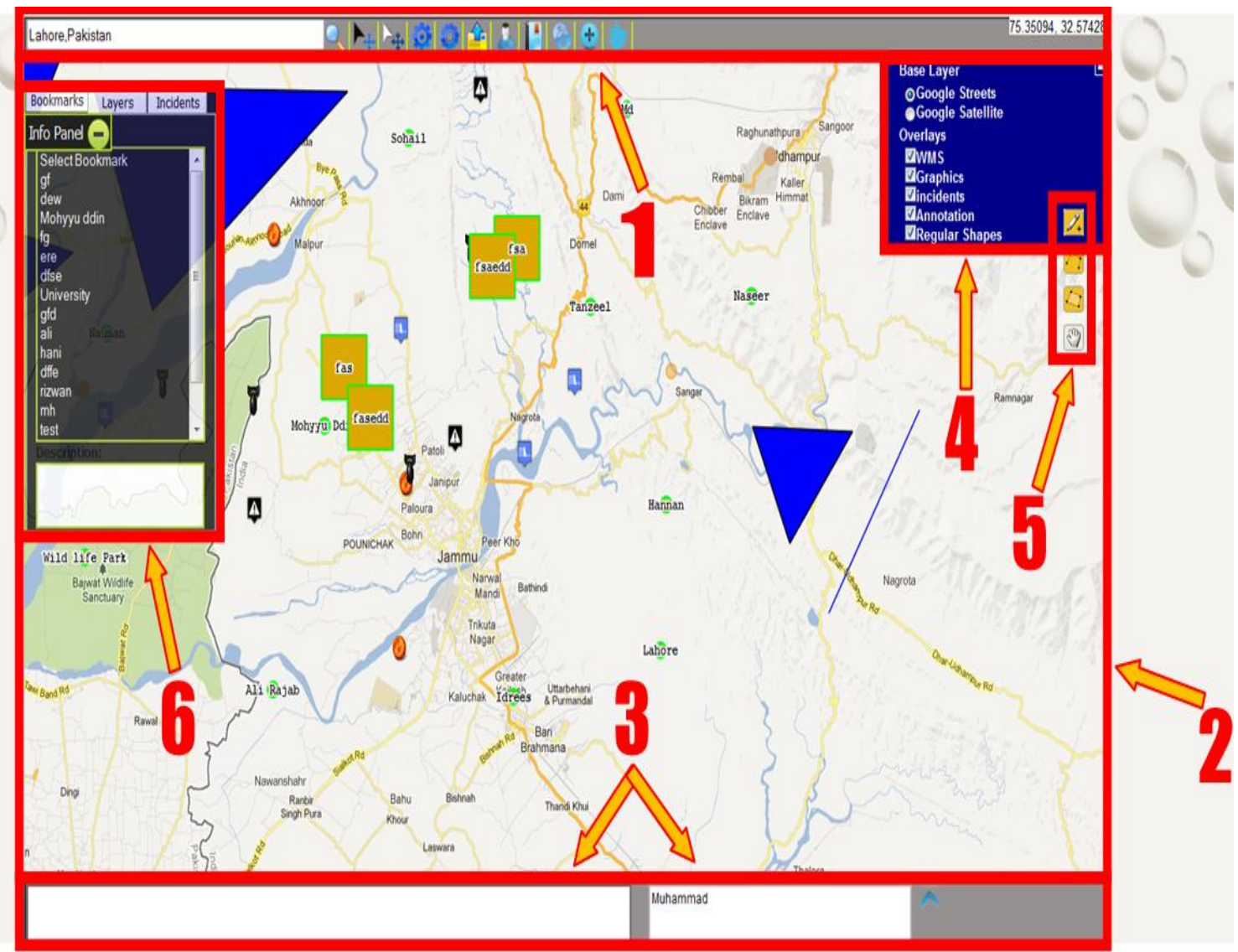

Figure 4.19: Co-PPGIS Map-sharing Component (online access at http://coppgis.com/geomeet4) 
One of the most crucial objectives of the Co-PPGIS map-sharing component is to provide an easy-to-use interface for inexperienced users to explore a spatial context collaboratively. This component interface consists of six major panels as marked (1 to 6) in Figure 4.19, which are discussed as follows:

1) Map Navigation Toolbar: consists of standard map navigation, floor control moderation, and some other mapping tools. Top navigation tools are briefly discussed and categorized as:

a) The standard map navigation toolbar provides basic functionalities such as searching, panning, and navigation. The search option provides a way to find a specific place of interest. The moderator can navigate participants to the area of discussion, or areas where some plans are portrayed to cope with different types of situations. All participant maps views will be re-centered or synchronized to enable the moderator and participants to discuss the same area.

Furthermore, a standard toolbar is provided with map zoom-in, zoom-out and pan functions.

b) Floor control moderation tools (i.e., deactivate floor, activate floor, request moderation, accept moderation) are used when a participant wants to hold a floor of discussion (as a moderator) and deactivate other participants' involvement on the map. Similarly, the moderator can show and hide the mouse pointer to others, which allows the moderator to point out certain features of maps and/or his/her point of view during the discussion.

c) Place marker/whiteboard tools are built for place marking in relation to municipal planning, such as marking a new roads network for controlling road congestion, specifying new park areas and/or school areas in smaller municipality on the map. Feature geometric shapes such as circles, triangles, pentagons, and hexagons, are also part of this place marker category, which is developed for depicting special areas of interest using advanced white-boarding techniques. Moreover, the bookmark tool helps in saving the particular area of the map with associated spatial comments and annotations, which allow the participants to share multi-user discussions with each other.

2) Map Viewing Area: covers a large portion of the screen and allows the meeting attendees to view spatial data, as well as associated comments and annotations.

3) Chat and Participant List Panel consists of two components: the chat component and the participants list. The chat component allows users to comment and have discussions with others while the participants' list window shows other active online participants in the collaborative meeting session.

4) Map Layers Selector Panel: allows users to turn on and off certain map layers in order to view overlaid layers clearly. During the collaborative participation sessions, map layers selection workflow is synchronized so that the changes happen on a real-time basis between different users. For instance, when user A turns the map layers off and on, user B must see the map layers' changes in real-time on his/ her Co-PPGIS map-sharing component 
interface. The base layer switcher allow users to switch between different base maps, including maps provided by OpenLayer, any Web Map Services (WMS) and the OpenStreet map. The editable overlays are the transparent layers storing textual and graphical annotations. The moderator is able to switch base map layers synchronously (e.g., to check or uncheck the radio button of each map layers switch base map view on real-time basis).

5) Standard Map Annotation Toolbar: includes point, line, polygon and panning tools.

6) Information Panel: provides the information related to previously stored bookmarks, map geometry features and android device-based marked areas.

\subsubsection{Walkthrough of Co-PPGIS Map-sharing Component Services}

In fact, map navigation tools provide the most powerful mapping functions inside the Co-PPGIS map-sharing environment related to its ability to annotate. This situation allows participants to get involved by entering their annotations and suggestions into maps; and in turn, developing a better understanding of spatial information. A walkthrough/demo of some of the processes in connection to the Co-PPGIS major map-sharing component's services (i.e., annotation and drawing new features widgets) is explained briefly as follows ${ }^{10}$ :

\section{i) Geospatial Bookmark Sharing Widget}

The functionalitiy of geospatial bookmark tool is similar to the bookmark feature available in commonly used Web Browsers. The geospatial bookmark is usually created by participants, and serves as a shortcut to a previously viewed geographic location. It is essential to post discussions on any issue as one can retrieve a map's geographical area linked with its annotation and description. The process for co-sharing map area with its annotation features and participants' discussion using the geospatial bookmark sharing widget is discussed as bellow:

$>$ First, the participant selects the area of interest on the map, and then, presses the 'Add Bookmark' button at the top of the map navigation toolbar.

$>$ Second, participants enter the bookmark information (i.e., title and comments) into the bookmark popup window.

$>$ Third, the bookmark title information is updated in the prototype information panel below the bookmark tab.

$>$ At the end, the map area is zoomed to the bookmarked map extent (area of interest) and shared with all the participants on a real-time basis.

\footnotetext{
${ }^{10}$ A walkthrough/demo in connection to Co-PPGIS major map-sharing components services can be viewed at the URL: http://coppgis.com/video
} 
The steps involve to bookmark an area of interest on the map using geospatial bookmark sharing widget is shown in Figure 4.20.

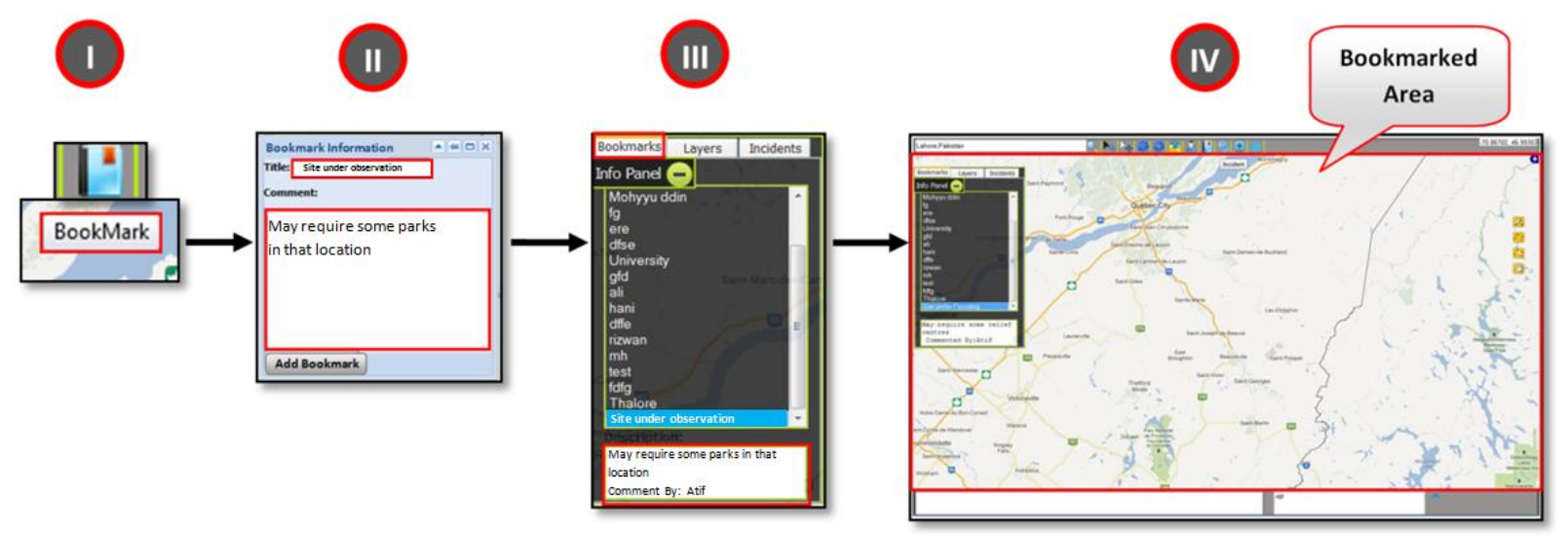

Figure 4.20: Geospatial Bookmark Sharing Widget

\section{ii) Incidents Place Marking Widget}

The process workflow for placing an incident marker into the map is as follows:

$>$ First, the user selects the 'Add Incident' button at the top of the map navigation toolbar.

$>$ Second, the user selects the area of interest on the map where he/ she wants to add the incident feature, then the type of incident feature (i.e., new park area, new hospital, parking lots, and amendment plan of a municipality region) is selected from the add incident features popup console, and finally, the description of the incident is entered. Once the user hits the add button, the incident will be added to the map view area, and the information list panel is updated with the title of the incident.

$>$ Lastly, if the user selects an incident title from the information panel, the map zooms to the map extent with the popup info besides the incident synchronously. 
The steps involve to mark an incident using place marking widget service is shown in Figure 4.21.

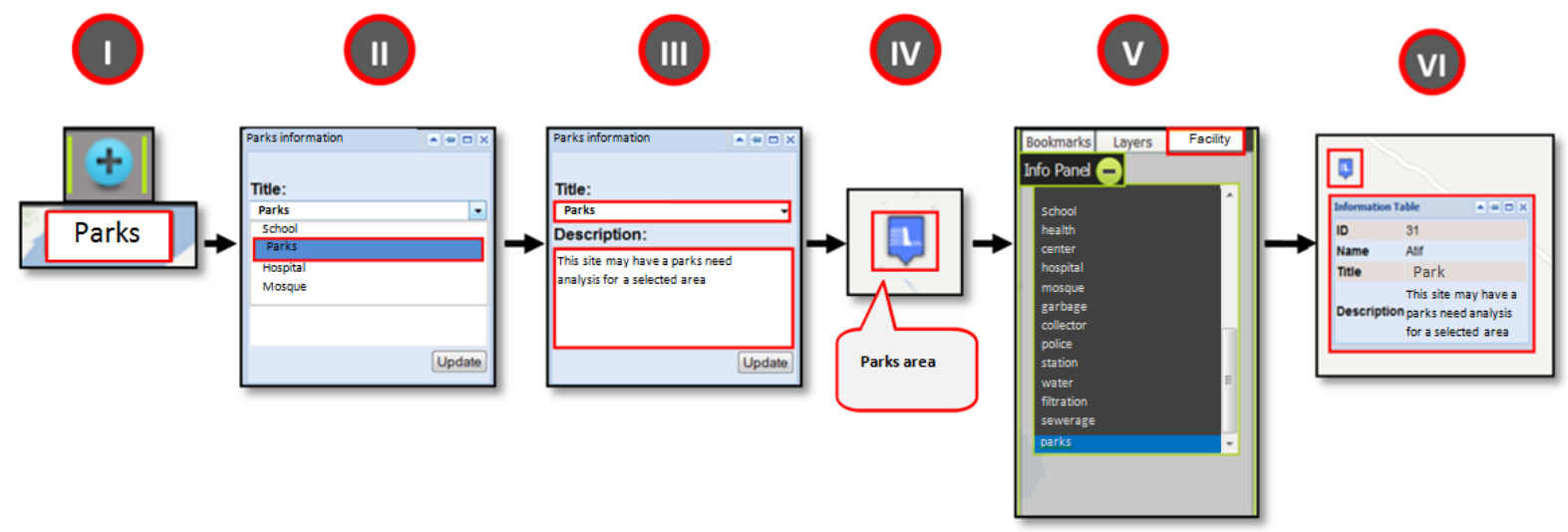

Figure 4.21: Incidents Place Marking Widget

The textual/geometrical annotation may be added at a transparent map layer, acting as a shared whiteboard. The annotations can be in the form of points, lines, polygons and place markers. These annotations are geo-referenced; meaning that they will be scaled as the map display is zoomed in and out resulting in no "distortion" of the annotation. The geo-referencing toolbar is designed and developed in order to depict the real-time supportive georeferenced based annotation on the map. The annotation tools are organized in a toolbar. To use the annotation tools, select the appropriate icon on the toolbar and then a location on the map to add a point and to draw a line or a polygon. The comment is then spatially linked with the user-defined drawing feature and stored in the spatial database. The Co-PPGIS map-sharing environment attendees may select a feature incident 'title' below the 'layers' tab of the information panel in order to view other participant's contributions in real-time. 
The steps involve to mark an incident using map feature geometry widget is shown in Figure 4.22.

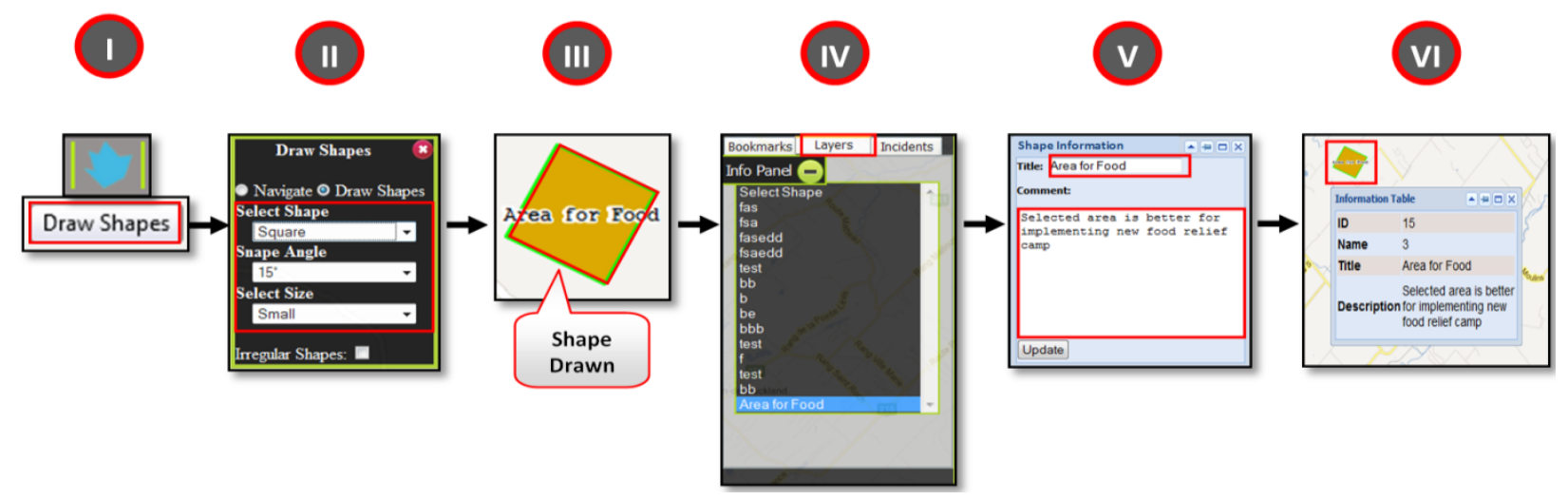

Figure 4.22: Feature Geometry/Shapes Widget

\section{iii) $\quad$ Android-based Geo-Tagging Widget}

The android-based geo-tagging concept is applied in the Co-PPGIS map-sharing environment. The use of an android device to annotate on the map and/or android geo-tagging concept provides another medium of communication among dispersed and co-located users. All types of stakeholders (i.e., field investigators, municipality management team, and government agencies,) who are involved in the planning and management process can remotely access the map-sharing interface, share and discuss their ideas, and geo-tag the areas of interest using an android device.

For example, after downloading and installing the MapSharing.apk application, which is developed to integrate android users with the Co-PPGIS map-sharing environment, to report municipal planning related issue instantly and to facilitate collaborative discussion. Each participant can mark a place on the map using an android device. Moreover, participants can visualize the android-based Geo-Tagging of other users concurrently within a shared map interface.

\section{iv) $\quad$ Floor Control Widget}

The floor control moderation mechanism enables the moderator to decide which participant has the privilege to take over control during the discussion. Floor control widgets (see Figure 4.23) are developed to avoid any kind of deadlock situation among attendees during the synchronous collaborative virtual meeting participation. The main idea is to encourage the use of moderation tools where two or more participants are involved in the discussion. The 
participant may require and/or want to become a moderator with full rights to draw and share contribution on the map and set other participants' rights as viewers only (i.e., all participation on the map is restricted).

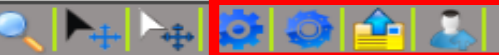

Figure 4.23: Floor Control Widget Toolbar

Detailed workflow of each tool on the floor control toolbar is explained in diagram (Figure 4.24- 4.28). The moderator can zoom/search for a particular municipal area within a city for geo-collaborative discussion. The Google map address geocoding API was used for the development of this search function. Mouse pointer tools are used for the activation and deactivation of mouse position (geo-referenced gesturing) on the participant's screen. The black pointer, 'show pointer' explains the moderator's action in order to be clear to the participants. It activates the geo-referenced pointer on the map that has a means of "gesturing to" the map features of other participants For example, the meeting moderator may need to point to the discussion place on the map. It helps in highlighting an issue which can be seen by all participants on a real-time basis. The white pointer, 'hide pointer', t.

hides moderation's movement of a cursor during virtual meeting. It deactivates the pointer sharing on the CoPPGIS map-sharing environment to other participants, which can be useful for single user/moderator work. It is very important for the participants to be able to view the moderator's actions (e.g., moving a mouse pointer to highlight the area of discussion). All other participants in the same virtual meeting session can see the mouse pointer alongside the user's information (Figure 4.24).

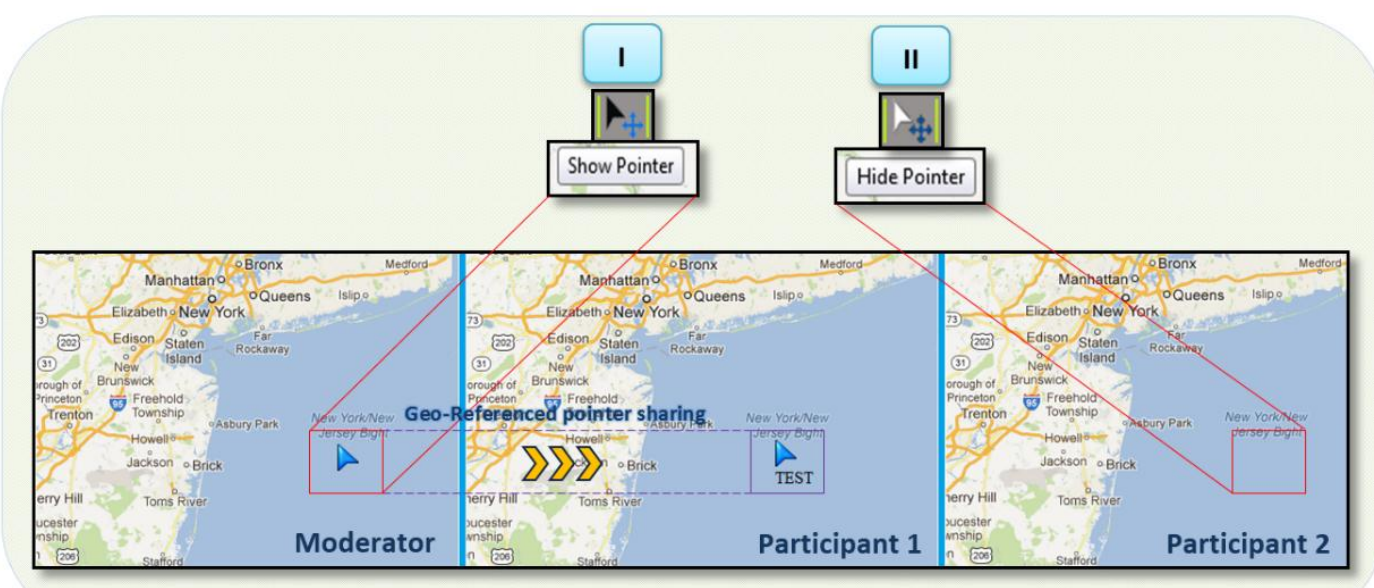

Figure 4.24: Show/ Hide Pointer Tools of Floor Control Widget 
Floor control tools (i.e., deactivate and activate floor) provide a smooth means of concurrent communication during the real-time virtual meeting session in order to solve any planning, management or decision-oriented problems instantaneously. Deactivate tools can be used by the moderator to deactivate participant tools (i.e., participant toolbars, map navigation controls, search, and other tools) during a discussion session as one of them acts as a moderator. The 'deactivate tool' can be of great importance during discussions because no participant will be able to disturb the moderator. Most collaborative operations, for example, viewing and zooming to the incident, making annotation, bookmarking and adding/ deleting incident, can be deactivated by the moderator using this tool. Other participants can see the sequence of actions performed by the moderator without any interruption during the geocollaborative discussion (see Figure 4.25).
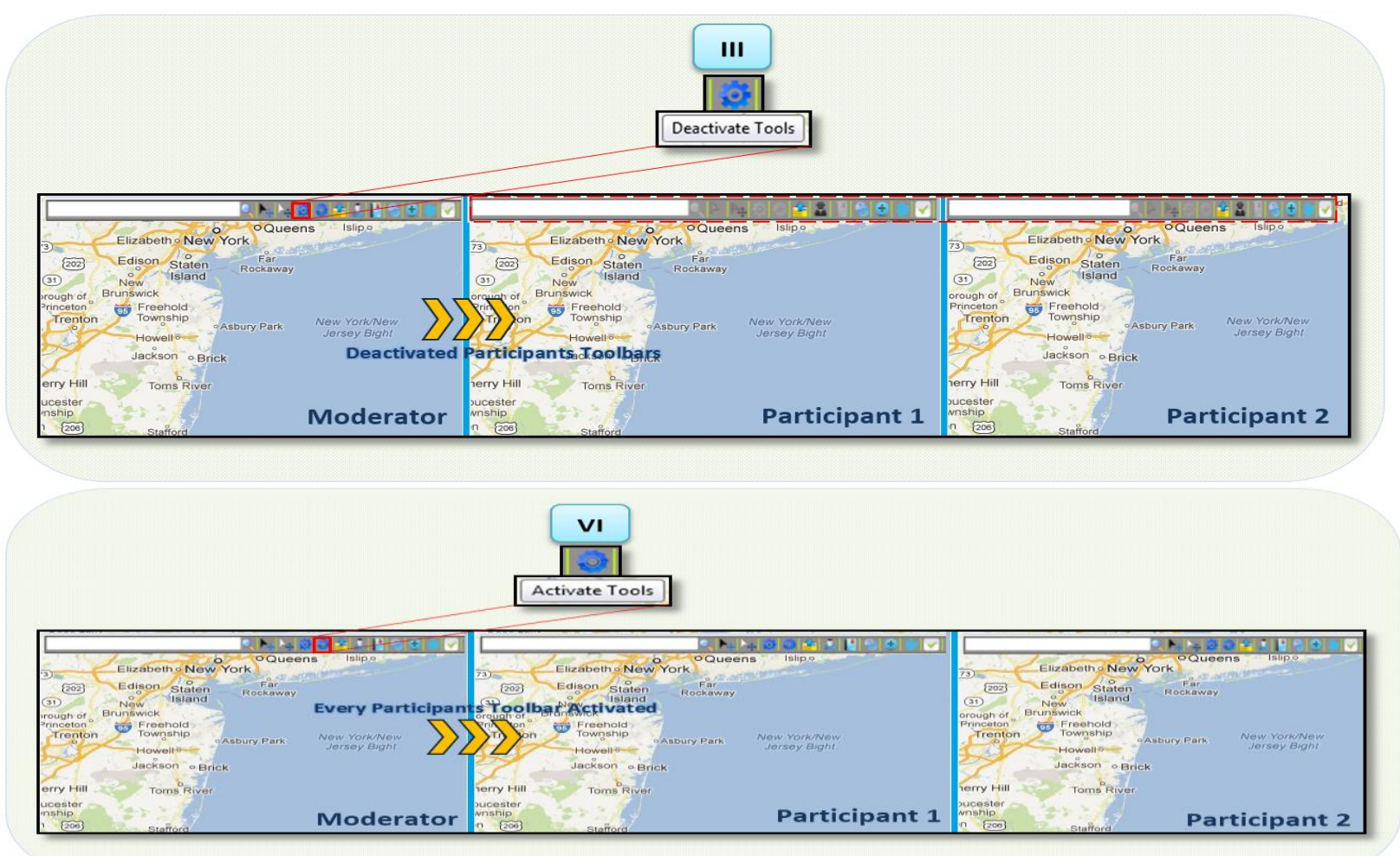

Figure 4.25: Deactivate and Activate Tool of Floor Control Moderation Widget

The 'activate tool' enables all meeting participants to see the moderator status throughout the meeting. During the deactivation status, participants can ask the moderator's permission to host the session. For example, if a discussion is underway and one participant wants to act as a moderator, the toolbars of the other participants are kept in 
deactivated mode. The send request tool can be used when one of the participants wants to interfere or give his/her suggestion regarding the ongoing discussion. The participant can send his/ her request to the moderator and give his/her feedback or suggestion. Requests are shown in the form of an alert box to a moderator by specifying which participant wants to give his/ her suggestion or feedback. In this scenario, the moderator can accept his/ her request by using the 'activate requester' tool that can activate the participant's toolbar. Figure 4.26 shows the process workflow in which participant 2 has requested the moderator's approval for his/her geo-enabled participation along with the workflow. In this case, the moderator has granted the right of moderation to participant 2, but participant 1 must keep his status in deactivation mode.
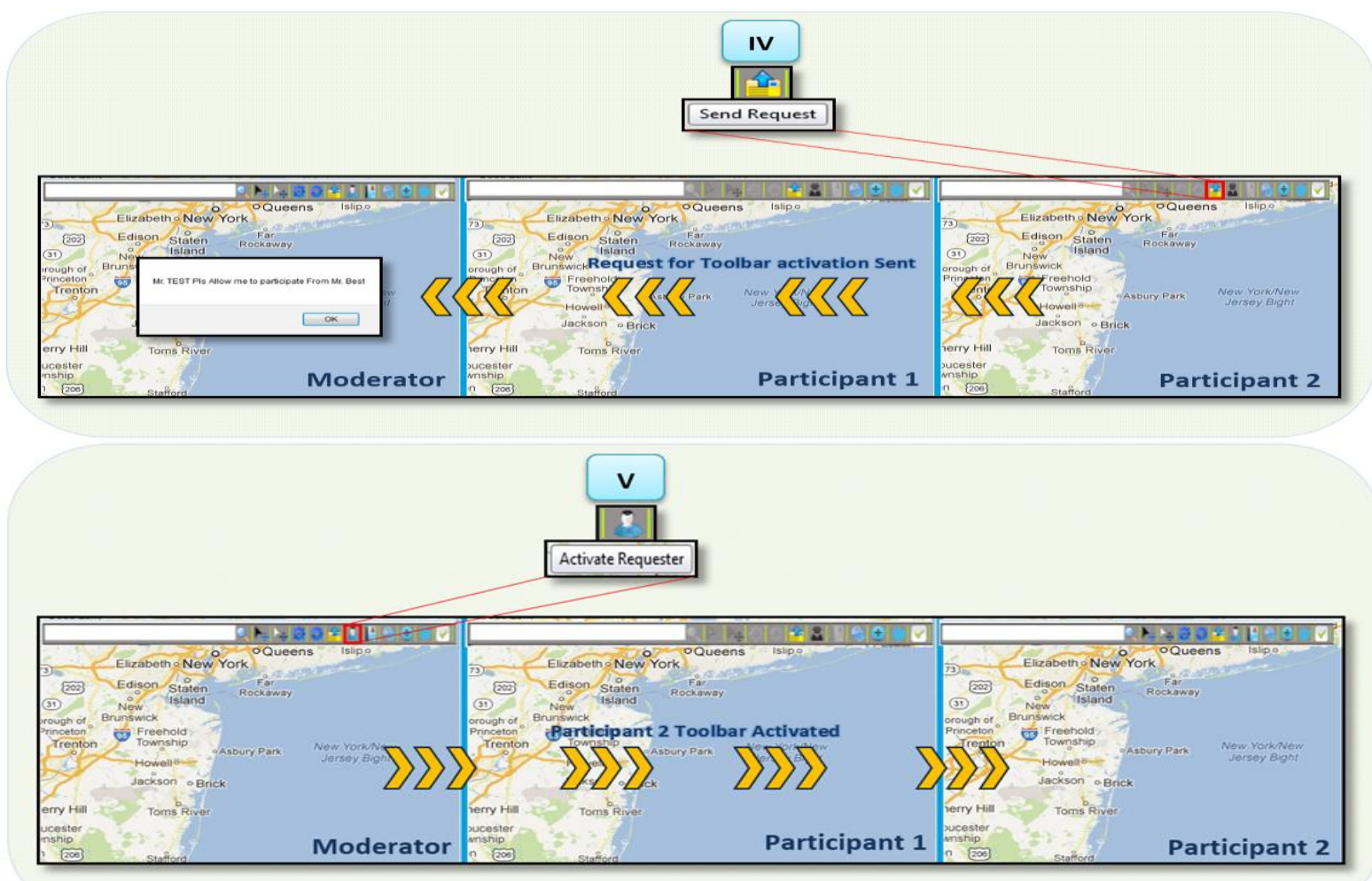

Figure 4.26: Send Request Tool and Activate Request Tool of Floor Control Widget

\section{v) $\quad$ Feature-based Chat Widget}

A number of ways that facilitate collaborative discussion among participants exist in the Co-PPGIS map-sharing component. Participants can put textual annotation on the features of a map. Another way is to make comments, or reply to comments made by other participants through the feature-based chat widget. This chat widget provides a 
useful chat function and the ability to view comments against features. During a virtual meeting, a user may be required to view the feature associated with a particular discussion. All messages are threaded against individual features (i.e., incident place marker, point, line and polygon); therefore, they provide rich discussion in relation to a particular incident marker. This process is one of the ways to build structured discussions, which are easily reviewed by other participants later/during the virtual session. The process workflow of a feature based chat widget is as follows:

$>$ In the first step, a participant must initially access the chat window by selecting the "Chat" tab within the identified popup window.

$>$ Second, the user enters a message against the feature drawn on a map.

$>$ After selecting the submit button, the message starts showing the information such as date, time and participant's name at the top of the chat window.

The procedure adopted to generate a response from the other participant is similar to that of other chatting software. For instance, the second participant can reply and post the messages on the chat window. Moreover, a reply message against the selected feature is automatically linked with the original message posted by last the user.

\section{vi) $\quad$ Feature-based Multimedia Sharing Widget}

The feature-based multimedia sharing widget is shown in Figure 4.27, which illustrates how multimedia objects can be added with a map marking tool (i.e., park feature). These multimedia objects can be viewed against attached feature.

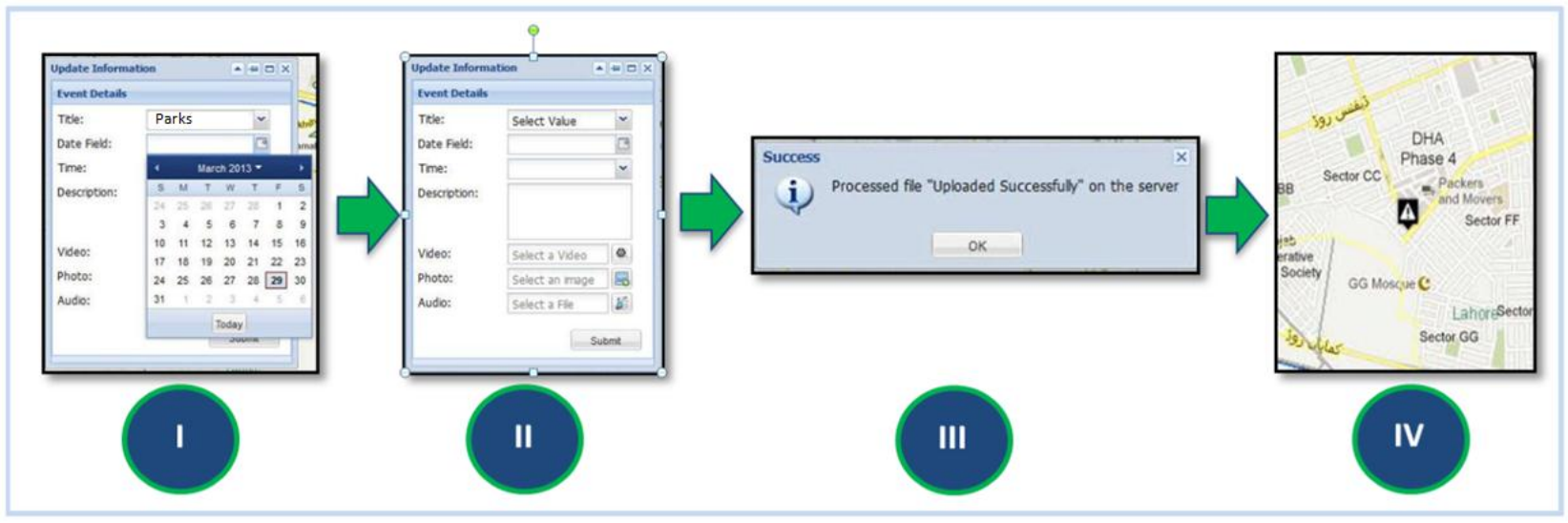

Figure 4.27: Feature-based Multimedia Adding Widget 
The feature-based multimedia sharing widget concept is similar to the idea of facilitating feature-based chatting in the Co-PPGIS map-sharing component that forms the medium of synchronous communication with customized, instant media sharing facilities. It provides a real-time collaborative environment between geographically dispersed participants, where each participant can GeoTag map features and share rich media (i.e., images, videos, audios and documents) and/or share messages with the other attendees of a meeting.

\section{vii) Incidents Query Builder}

The incident query builder of the Co-PPGIS map-sharing component is designed to allow multiple people to view the results of particular incident occurrence within a specific period. Participants can find any type of planning issue (e.g., incident markers placed for the requirements of new park's area in the municipality since over the last four months). Each query result is spatially linked to the incident marker geo-tagged on the shared map and provides a collaborative way for discussing spatially-sensitive issues.

Figure 4.28 shows the process of query builder tool, which require in order to show incidents (i.e., park needs) within specific dates.

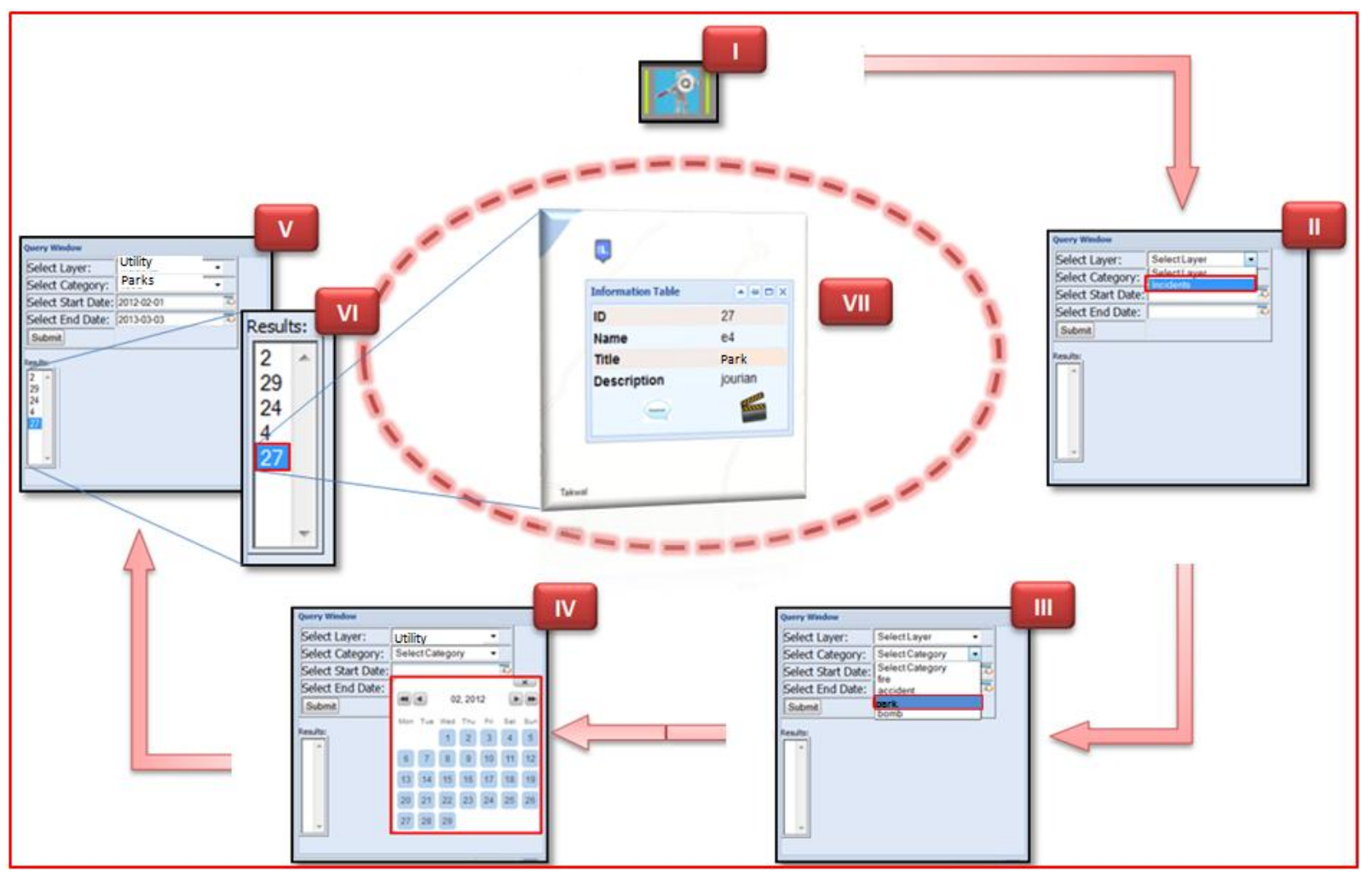

Figure 4.28: Process of Showing Incidents Query Builder 
The objective to develop this incident query widget is to facilitate participants to perform query-based analysis and knowledge mapping by interchanging views quickly on critical planning issues.

\section{viii) Incidents/Markers Report Builder}

The real-time incident reporting dashboard concept is implemented in the Co-PPGIS map-sharing component with a comparison graphs support widget that forms the medium for comparative analysis of graphical results among dispersed or co-located users. The incident report widget enables meeting participants to visualize the results of incidents marked on different dates simultaneously within a shared map interface. These incidents are geo-tagged by different participants using two types of component interfaces (i.e., Co-PPGIS Android devices-based application and map-sharing Web interface).

Figure 4.29 shows a report that is published based on incident marking, such as need for parks, existing parks, and children ratio to go in parks.

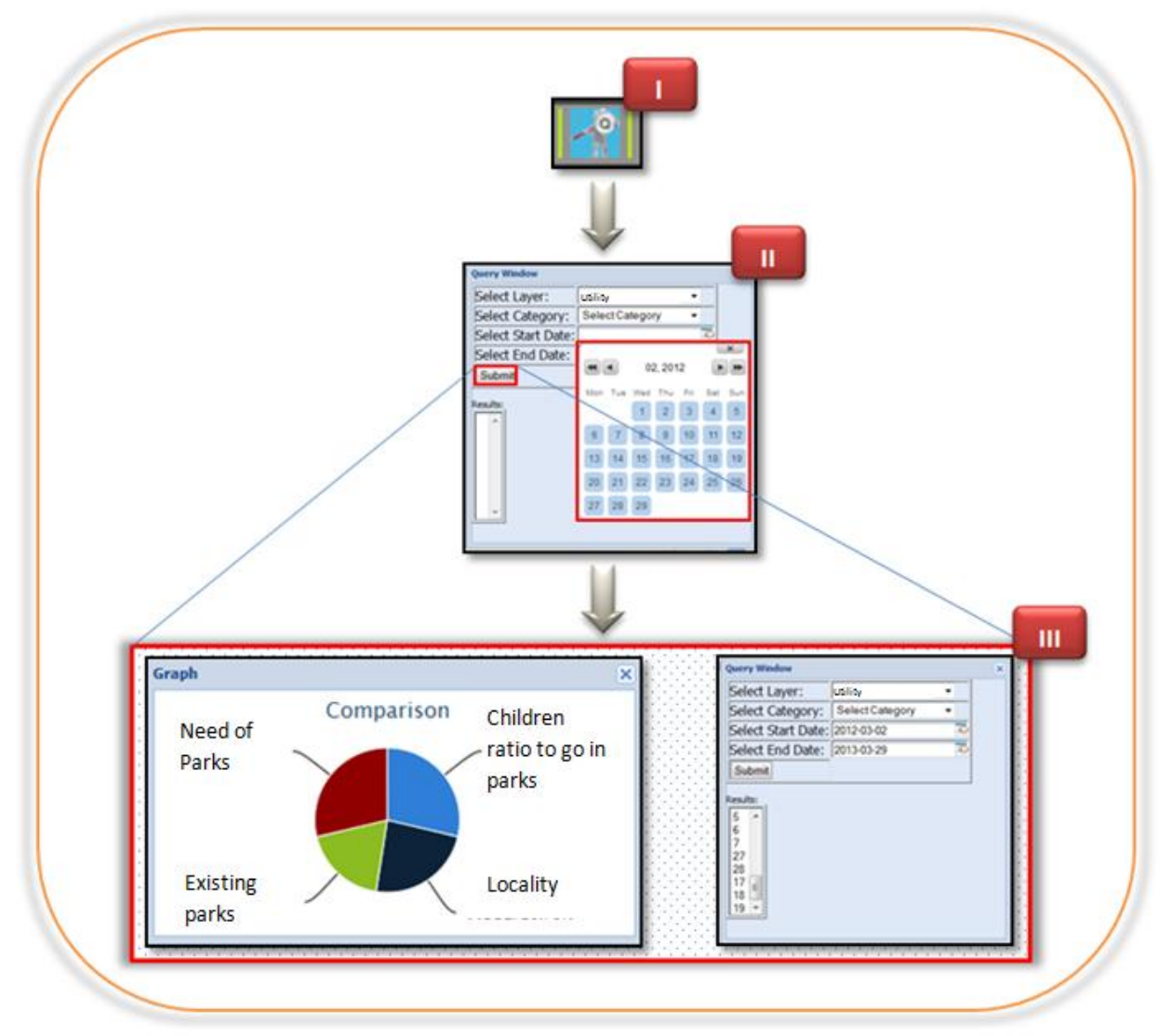

Figure 4.29: Process of Showing Incidents Report Builder 


\section{ix) Live Video Sharing Widget}

The live video sharing widget is an add-on to the Co-PPGIS map-sharing participatory environment, which is developed to provide a real-time, multi-user video conferencing environment that offers live video communication among different participants. The video sharing widget is designed from the ground-up to take better advantage of Flex, JAVA and Red5 open source video streaming server technologies. This modular implementation allows and/or shares live videos of geographically diverse participants, which results in a very dynamic, interactive, and communicative Web interface (Figure 4.30). This component can be enabled or disabled during the spatiallyenabled discussions using the Co-PPGIS synchronous and asynchronous map-sharing participatory approaches.

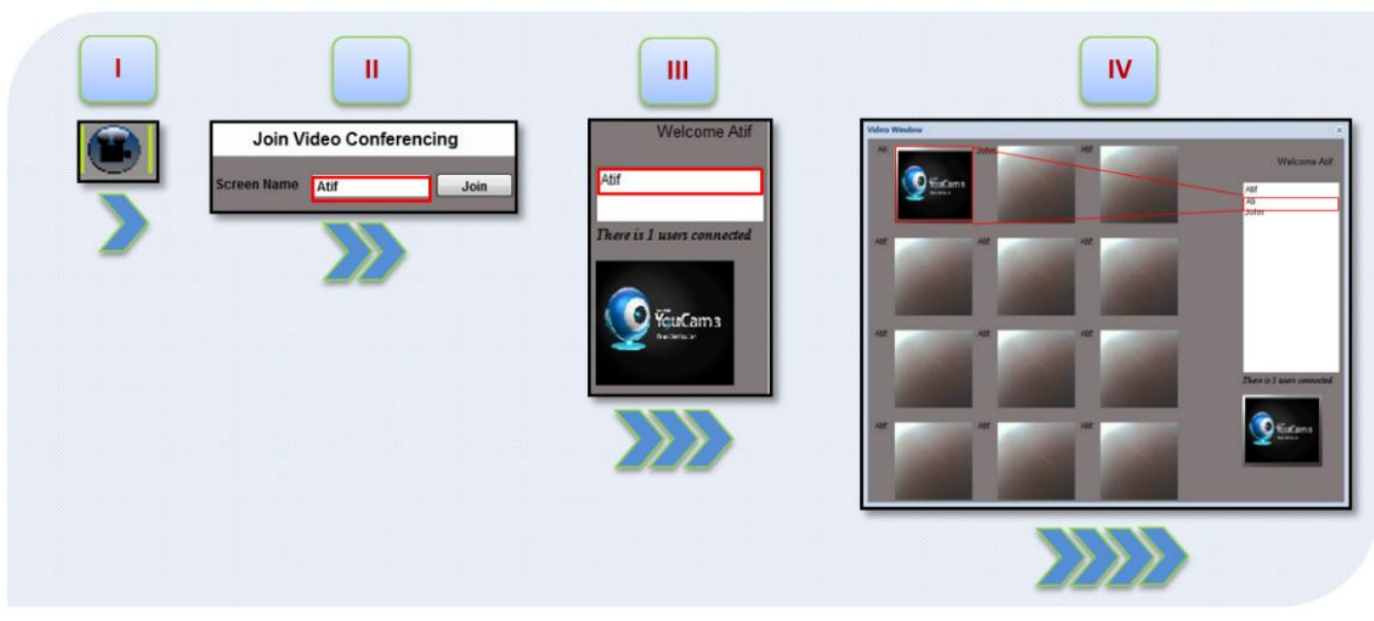

Figure 4.30: Process of Enabling Video Camera Popup during Co-PPGIS Map-sharing Participatory Environment

\section{x) Login and Room Console Management}

To integrate an authentication method a new login process is designed. The login process is required to support a room mechanism as well as registered user authentication through a local database (of PostGreSQL). If the user is not registered, manual registration can be done; however, valid credentials are required to access this virtual room interface. The main objective of creating different sets of rooms is to sort out the discussion according to the topic. For example, the moderator can set up a town planning related discussion in room $\mathrm{A}$, while a discussion related to a region amendment can be set up in room $\mathrm{B}$. The login and room process mechanism ${ }^{11}$ of Co-PPGIS is developed using Flex, ActionScript, and PHP programming languages.

\footnotetext{
${ }^{11}$ Prototype developed using a login and room process mechanism can be accessed using URL: http://coppgis.com/coppgis

For example, username: admin password: admin has the privilege to enter in any room whereas username: test password: test has the privilege to enter in room 3
} 


\subsection{Demonstration of the Prototype using Mock-up Scenarios - Co-PPGIS Implementation in Planning and Management related Activities}

The Co-PPGIS concept can be used to resolve a wide range of current global problems. The system may be applied to cope with decision-making in the planning and management of resource allocation. For instance, it may be a helpful to many applications, such as public health security, environment monitoring, and municipal planning and management. This is only possible when a system like Co-PPGIS imparts information on a 24/7 basis. It is then managed and dispersed to every responsible authority through a virtually linked network.

In the following sections, the Co-PPGIS prototype is applied to different areas of study (e.g., affordable housing, land use planning, and parks and recreation). The examples come from mock cases or scenarios developed in different regions of the world.

Scenario 1: Co-PPGIS - Provides Measures for Transparency in Private Affordable Housing Schemes and Sustainable Master/ Land-use Planning

The colonization of completely developed housing schemes can contribute not only to meeting the housing backlog in major cities of the world, but also to effective utilization of infrastructural services supplied several years ago. Such growth may lead to irregular urban sprawl, ultimately affecting living standards in terms of provisions and infrastructure. The provision of better quality of life can be improved through private housing schemes that play a pivotal role.

To regularize schemes according to land use rules, authorities keep development Master Plans in mind. Private housing schemes are enforced to register before launching their service and are compelled to abide by rules approved by the competent authority. In the current scenario, meetings are called upon on short notices. Stakeholders are congregated at specific offices to examine housing schemes at different times depending upon the suitability. This may be a waste of costly time for both beneficiaries and decision makers.

The idea of implementing Co-PPGIS as a Web-based synchronous and asynchronous map-sharing solution may result in a unique and rapid way of real-time communication for geographically diverse stakeholders. Only short training can make them adapt to the system. Participants are able to share views as they would in normal meeting sessions by identifying specific events that can be viewed by all. Specific layer options like housing boundaries can also be made active for the discussion (only one layer can be active and analyzed at a time, as at this stage tool is not supported for multi-criteria analysis). It carries information at plot level and gives information about the town name, owner name, picture of the house, plot number and concerned authority name. Status of all existing and ongoing 
projects in the housing sector in the form of tables, thematic maps and reports may also be included. Teleconferences in both media (audio/ video) can be conducted. Figure 4.31 epitomizes the private housing scheme. Blemishes are identified with the help of the development authority and visualized with the help of the Co-PPGIS component for decision making.

Figure 4.31 depicts the private housing schemes data on a shared map view using Co-PPGIS component, which is developed for municipal planning and management related activities.

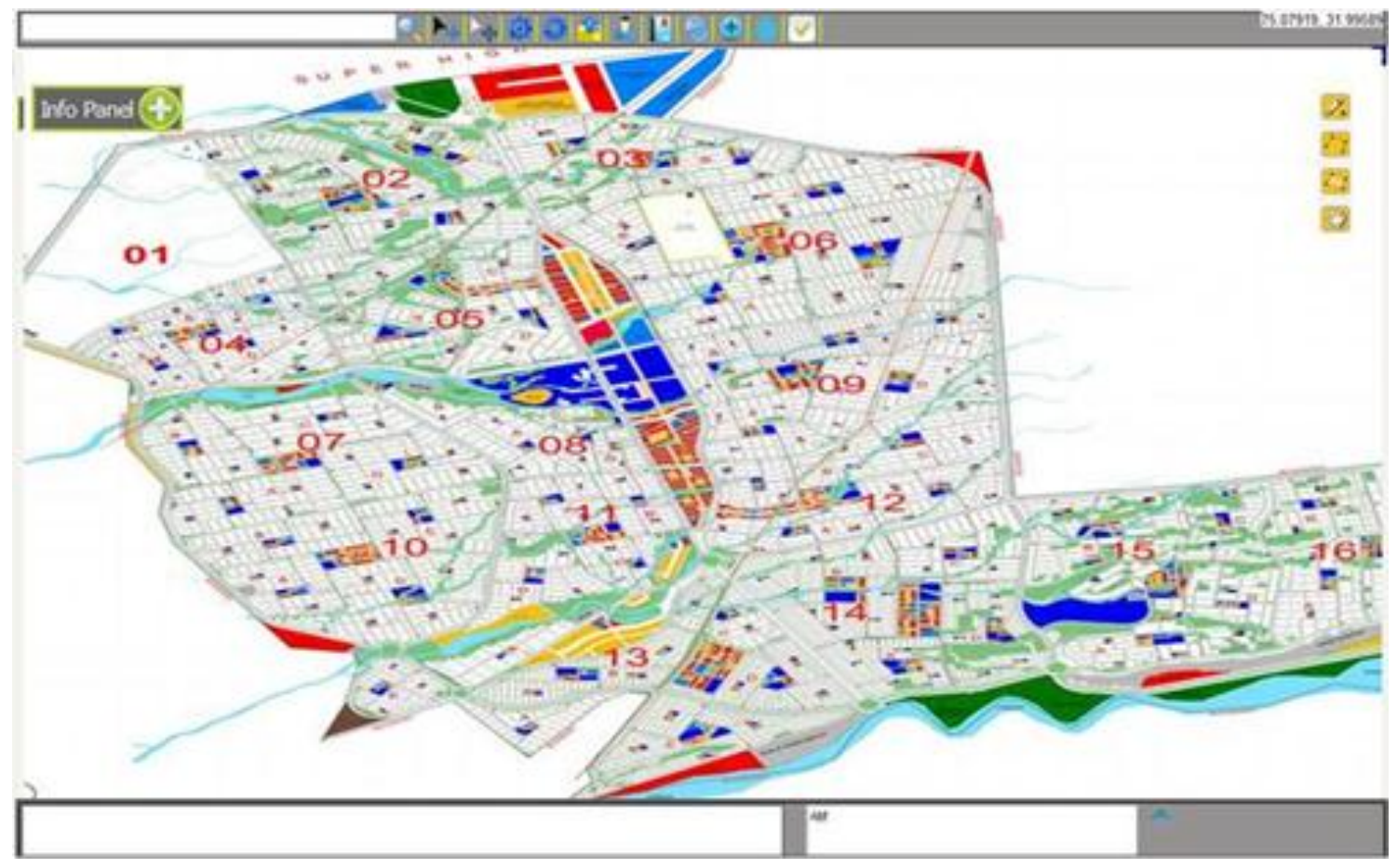

Figure 4.31: Co-PPGIS for Municipal Planning and Management

High-resolution satellite imagery related to the study area can be added for a better understanding of the housing scheme as a map layer. Data provided by the Development Authority can be added as a WMS (a base map layer) in Co-PPGIS, on which meeting attendees can discuss and present their results regarding housing schemes, development of new schemes, number of occupants and so forth. Different color symbols can be used for this purpose. For example, red shows commercial, blue shows residential and brown shows the vacant areas on the map where a housing scheme has been approved. An area labeled as ' 01 ' shows a proposed extension for a scheme in the future. An Android field survey can be helpful for individual plot identification. These points are then displayed on the base map with its location, picture and tabular data for discussion. 
Likewise, a master plan is the basis for the planning and construction of population centers. The ever increasing population and limited space within municipality jurisdiction cause challenges for concerned development authorities in terms of sustainable land use. Planning ahead for balanced land use needs is crucial for developing nations. Long-term strategic plans and detailed local plan preparation require a comprehensive, centralized and integrated planning approach to be adopted by higher officials in the light of public concerns. Sharing of land information, along with public agency requirements, act as base for preparing a master land use and local-area plan (see Figure 4.32). In the project team for master planning, several departments have to be organized with experts on subjects of spatial, transportation, social and environmental planning.

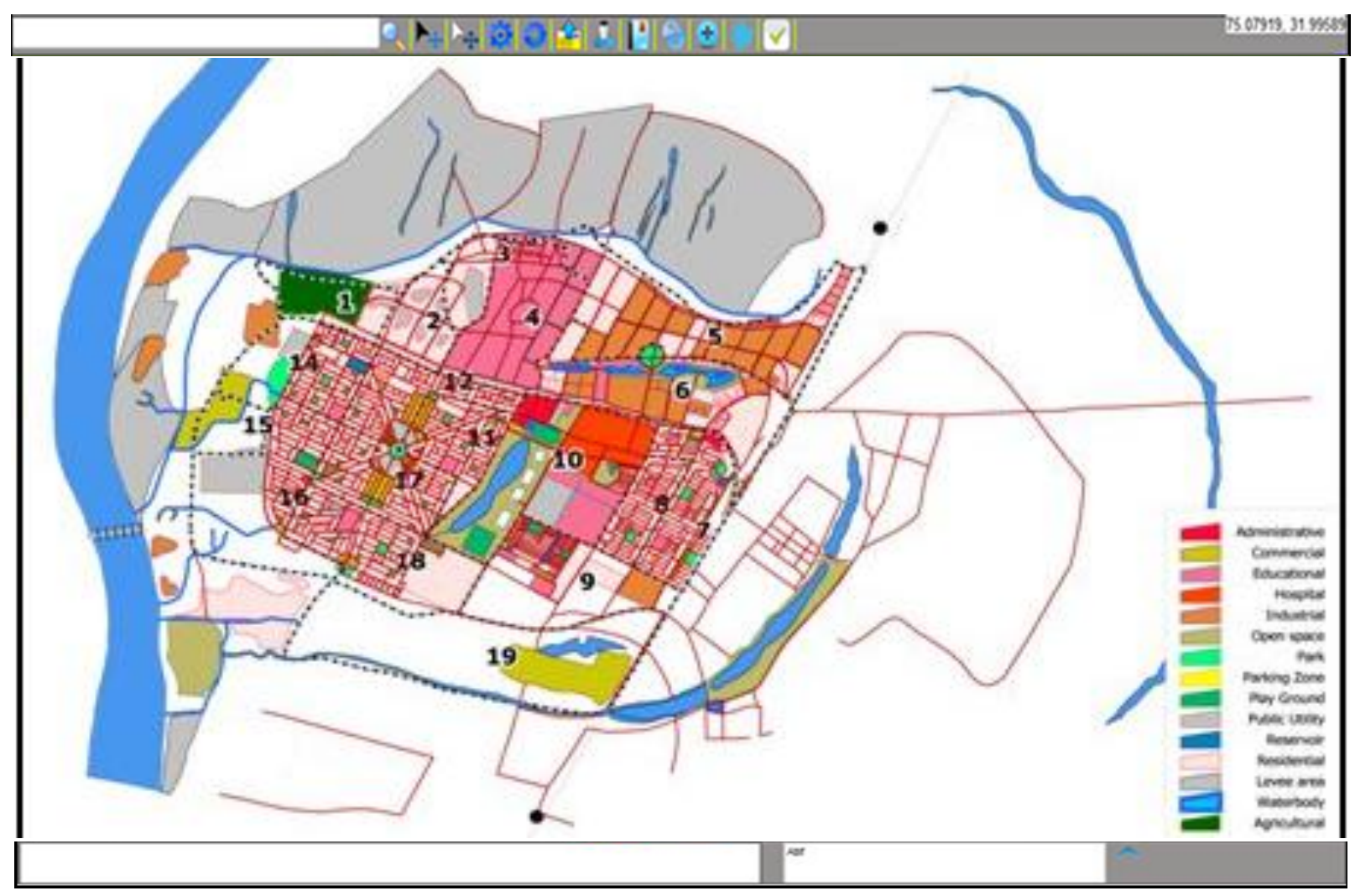

Figure 4.32: Co-PPGIS for Preparing Master/Land use Plan through Collaborative Approach

Using the shared geospatial data repository, along with Co-PPGIS, development authorities can create and edit the plan as well as publish the plan on the collaborative platform for viewing by general public. Online publishing of map to invite public consultation as easy access to master plan allows easy and effective participation in the land use 
planning process. The master plan can be improved after incorporating relevant feedback and suggestions by the public and stakeholders.

\section{Scenario 2: Co-PPGIS - How Well Municipalities are Meeting the Need for Parks}

Parks are usually characterized by a large public green area in a town that is used for recreation. Parks are important to communities because nearby opportunities to exercise and experience nature are essential for physical and mental well-being. Studies show that parks encourage physical activity, revitalize local economies, and help bring neighborhoods together.

There is a need to sort out park areas; and in turn, improve local living standards and lifestyle. The government is responsible for identifying and creating easy access to necessities to life-like national parks, green infrastructure, recreation and culture. Distributed park data is available and is being maintained by government bodies; however, they are insufficient in order to meet the current challenges.

A Web-based system has been launched to handle and share data in real-time. Co-PPGIS is a way to have collaborative meeting sessions at regular intervals. A set of tools like point helps to identify park areas and underlying base map information about infrastructure. Geo-teleconference and/or video or audio-based map-sharing makes it possible for decision-makers to make a decision quickly. All the map feature editing related to the identification of potential park areas, which is made by the presenter, can be shown to all participants at the same time in order to facilitate collaborative decision-making.

The presenter can mark a place, as well as add comments and information regarding a citywide analysis of park access by age and income level. They can also add detailed information about each city park, including: data and analysis pinpointing where parks are needed most critically and identifying which improvements would provide the greatest benefit to the local park system. The boundary of a park area can also be attained and shared real-time with all participants. 
Figure 4.33 represents the identified areas to show the levels of park need and/or levels of satisfaction with existing services a Park District provides.

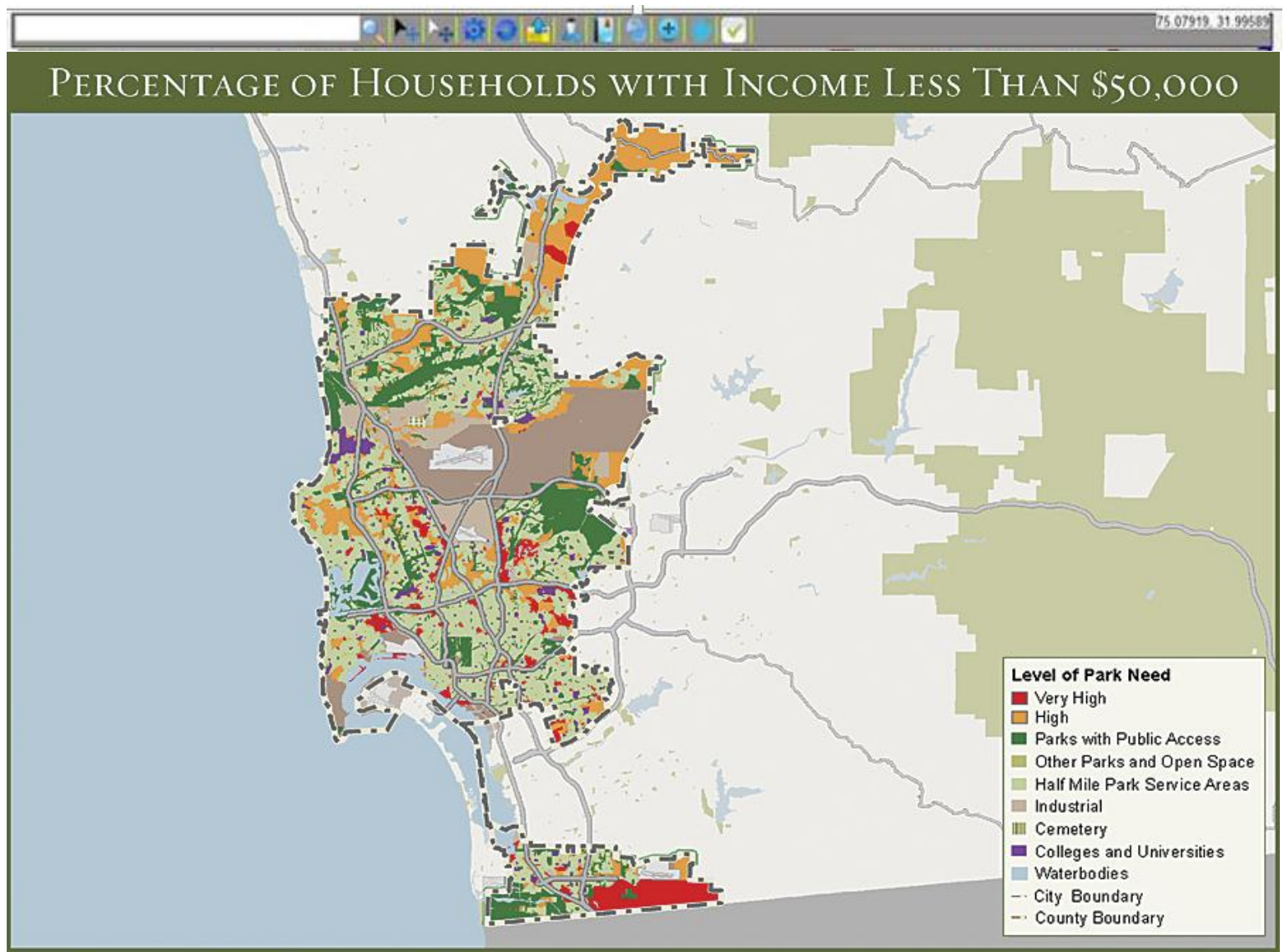

Figure 4.33: Co-PPGIS for Suitable Park Area Identification

Park areas are identified (as shown by points) using GIS land use survey techniques. Android-based techniques can be used for park point collection in the current scenario, which is a built-in functionality of the Co-PPGIS android component. This Web and android voting-based marking information is saved into a database and can be retrieved to make a thematic map (see Figure 4.33, in which red area indicates the high level of park demands). In addition, high-resolution satellite imagery is used for discussion as a base map so that parks can be easily viewed in high resolution imagery.

The Co-PPGIS environment creates a platform where stakeholders can share and implement their views related to area upgrades, including: providing better utilities, facilities, and living standards to residents. 


\subsection{Discussion}

This research study introduces collaborative methods and processes to encourage participation approaches that enhance public participation during the planning, development, and management situations. Co-PPGIS is an innovation based on technologies, such as groupware, CSCW and a real-time map-sharing mechanism, which support better decision-making through its innovative CSPE and CAPE-based map-sharing component technology. Co-PPGIS is a Web-centered system application that facilitates real-time public participation for group decisionmaking that ultimately brings limpidity in any system of e-governance, planning, and management. Co-PPGIS virtually connects public response and/or feedback with government agencies/bodies, such as municipal/urban planners, the ministry of regional development and public works, and the ministry of public health and safety. Consequently, Co-PPGIS provides a virtual platform for collaborative participation, where possibly many stakeholders can share their knowledge to build a consensus and to improve public participation. In brief, public response is the key to increase participation for better decision-making.

The incremental design and interoperable development approach to demonstrate the success of the Co-PPGIS-based concept introduced in this research study provides a guide for further research. There are some key design and implementation issues related to the Co-PPGIS prototype, which were identified and resolved during this research study. Some of the problems and issues are discussed briefly as follows:

\subsubsection{Intervening Problems and Issues}

Some of the social, design and implementation issues faced during the research and prototype's development are discussed in the following paragraphs. One of the main implementation problems is to find the role of an administrator/person/group before the deployment of the prototype in a real-world scenario. Ideally, such PPGIS applications should be run and maintained by the planning and development authorities, municipal government, and consultants in order to load required data and information, and the government agencies remain responsible for broadcasting public notification and for public meetings. However, this issue has not been dealt with in the current research; therefore, there is an immense need to actively involve end-users/ stakeholders at later stages of the research for further investigation.

The research also considers some sociological issues and forthcoming risks, which may occur after the system deployment. For example, stakeholders having difficulties accepting and responding to the Web GIS, multimedia and groupware technologies in connection with traditional participatory practice may be an issue. These potential hindrances will also lead to the use of the modular/component-based design implementation, which may be helpful 
in acquainting stakeholders to the new technologies. However, this issue might require further research and inspection during the later stages of the study.

There is also a need to deal with the issue related to access to information and confidentiality during the development process. It necessitates the implementation of protocols that manage the confidentiality of municipal project data (see Section 1.1.2) that is protected under copyright law. Setting the rights and privileges of the stakeholders at different levels of their interaction within the system may resolve this issue.

One of the forthcoming societal issues related to system prototype development is to accommodate stakeholders with disabilities. Addressing such an issue may require comprehensive research on its own, which has not been dealt with in the current research. Further discussion will be included in recommendations for future work to outline a potential expansion of the current research.

\subsubsection{Technological Issues}

As a research prototype is an integration effort from a technological perspective, some key design issues related to technologies used during the development of the prototype are described as follows:

During system development, special attention was taken during the selection of enabling technologies as it played an important role for increasing the throughput (quick response) and design/maintenance cost of the system. For example, spatial data was handled by an open-source PostgreSQL, object Relational Database Management System (RDBMS) that supported broad scale-interoperability with spatial data handling using a PostGIS extension (Sano et al. 2003; Wangmutitakul et al. 2003; Wangmutitakul et al. 2004; Wuttiwat et al. 2003). Although at this stage not all the prototype's components were demonstrated by using spatial database (PostGIS) due to the lack of availability of the spatial data related to municipality planning and development projects.

The collaborative map-sharing component has initially been designed for enabling a "spatial" virtual meeting space for public participation along with Web GIS support. This component has been developed using Google Map API, Adobe Flex, and Flash Collaboration Service technologies. The Adobe Flex platform provides and builds real-time collaborative applications rapidly as it offers a complete Software-Development Kit (SDK) that contains ready-touse components designed with collaborative workflow. The first issue at this moment is that the flash collaboration service in the Adobe platform does not support screen or media sharing protocols (another design challenge, which was not considered important during the initial development). The purpose of this function is to record the session 
history during the public meeting seminars or conferences so that it can be replayed and shared later for future events/discussions. However, this issue might need further investigation.

The interactive virtual public meeting component, which has combined functionality of collaborative map and document sharing, has been designed and developed using JAVA and Adobe Flex platforms. Integration of these two platforms was a big challenge for their suitability, connectivity and scalability in the beginning of the development. For instance, Flex code requires a flash player to run or execute; whereas Java requires Java Virtual Machine (JVM) to execute the compiled code. Other problems common to all online application developments are the compatibility of different web browsers (i.e., Internet Explorer, Safari, Mozilla and Google Chrome, etc.) and Internet connection speed. Using the JAVA platform will resolve the issue related to the screen and video sharing mechanisms of Adobe flash collaboration services. Therefore, the share screen module has been developed and added as a recording function along with a meeting planner component using JAVA as a part of the virtual public meeting interface.

Handling map-sharing features, such as mouse synchronization, was a big challenge as some of the open-source modules are not fully supported by each type of browsers. This issue might require further investigation and expertbased testing.

Initially, the mouse pointer was synchronized with pixel value that uses screen coordinates for pointer movement on a map component. The technique works pretty well for the same resolution, but when it comes to higher or lower resolutions the pointer on the participant's screen shows the wrong geographical area. This issue was resolved by justifying the map panel to the left only. However, the problem remained the same when placed in the center and/or right alignment, so the screen coordinates were made to initialize from the top left corner, and will remain the same for all screen sizes. Furthermore, another problem arose when we aligned the map-sharing panel on the left-hand side of the screen, there was a lot of vacant space generated on the right-hand side when participants used a larger screen resolution. This issue was resolved as the mouse pointer was synchronized with a map coordinate transformation technique instead of using screen coordinates, and then, each participant got the same geographical area as the moderator.

If a discussion with more than one participant is in progress in a virtual public meeting scenario, there might be a chance that they will change the map extent (area of interest in map) from its default location according to the need of the discussion. Later, if a third participant joins, he gets the default extent shared by the two initial participants. In the beginning, the prototype was designed by implementing a rule of thumb that once the meeting starts, no one was 
allowed to join the meeting session. Therefore, the default extent of every participant was set to be the same deliberately. Afterwards, it was realized that it would be better if we let late comers in and give them a chance to participate. This creates the need to share the last saved extent between different participants. In this way, saving previous extent state becomes another functional requirement recognized during the in-house version management of the Co-PPGIS application. This issue can be resolved by making an extent.txt file, which saves the last extent (latitude, longitude and zoom level) used by the participants already in the discussion. Thus, by retrieving coordinates parameters from the extent.txt file, when a new user joins the Co-PPGIS session, he/ she gets the same extent as the other participants.

A floor control tool was developed that grants/revokes permission to users to participate during the virtual meeting. In this prototype, everyone can be a moderator and can disable other participants' toolbar. A similar kind of issue is found among the participants who join late. The issue arises when participants are in discussion session and one of them is a moderator who has disabled the other participants' toolbar. Meanwhile, a new participant joins the discussion but his/ her toolbar is found enabled as there is no scenario programmatically handled for late comers. Initially, the prototype was designed without any specific moderator. Everyone could become a moderator and communicate manually by sending a chat message to other participants (e.g., "I am going to become a central moderator, and you all can just watch and don't need to interact until I finish my discussion"). It works fine, but it was noticed during the prototype in-house testing that the floor control moderation should be automated. To resolve this problem, a new field was added in the database with the name 'status flag' in which the status of every participant is stored. 'Enable' represents an active participation mode and 'disable' means that participation mode is in a deactivated state. Programming logic is designed so that the system checks a status flag on new participant's arrival.

A browser's CSS compatibility is one of the issues faced during the development. 'Chat window' and 'user list panel' work properly in Internet Explorer and Firefox; whereas it was not displayed in Google Chrome due to some unidentified error related to CSS. This issue might require further investigation and expert-based testing.

The editing toolbar is the main component of Co-PPGIS. Previously, annotations/shapes were not visualized immediately or simply vanished. This was a problem of Web Mapping Service (WMS) of Geo Server. It did not allow the geometry to be visualized properly. As a solution, WMS layer was refreshed using open layers "redraw layer" function by applying a JavaScript timer method. It refreshes the database after every drawn feature to make it visible to all participants. A consistency check service was developed to provide uniformity in sharing map extent to all participants involved in the discussion and retrieval of spatial context. During the map-sharing collaborative 
virtual session, map extent parameters such as longitude, latitude, and zoom level are recorded in the system file. The system file with these parameters is later shared with the newcomers so that they can see the synchronized geographical area as well.

\subsection{Summary}

This chapter starts with a brief discussion related to the Co-PPGIS prototype applications by highlighting its synchronous and asynchronous participatory approaches. The Co-PPGIS advanced map-sharing component along with a walkthrough of its component services are discussed followed by the demonstration of the prototype using mock case study scenarios and discussion summary.

Next, Chapter 5 covers a complete process of workflows/lifecycle that were adopted during the usability testing and evaluation of the prototype's framework through a case study of the York region in Toronto, Canada. 


\section{Chapter 5 Usability Evaluation of Co-PPGIS using Mock Case Study}

This chapter evaluates the prototype's implementation based on a number of aspects, including intended use, interactivity of user interface, exchange of information with GIS-based mapping functionalities, educational use, and feedback. A real-time collaboration application requires steady usability evaluation to ensure its workability for real planning situations. For a system such as Co-PPGIS, with real-time synchronous and asynchronous collaboration and integrated and developed with Internet-based PPGIS and open source technologies, its usability determines how effective it can help enhance collaborative participation as well as improve the decision-making process in any planning and management workflows. The chapter starts with a brief explanation of the proposed usability evaluation methodology, followed by a discussion of a mock case study.

\subsection{Proposed Usability Evaluation Methodology for Co-PPGIS}

The evaluation of the usability of Co-PPGIS prototype follows the following five methods: i) design and development phase testing or designer/developer testing approach, ii) intended user testing approach/user-based testing, iii) questionnaire/interview-based testing, iv) analytical testing, and v) statistical testing. Figure 5.1 depicts flow diagram for the usability evaluation of a Co-PPGIS.

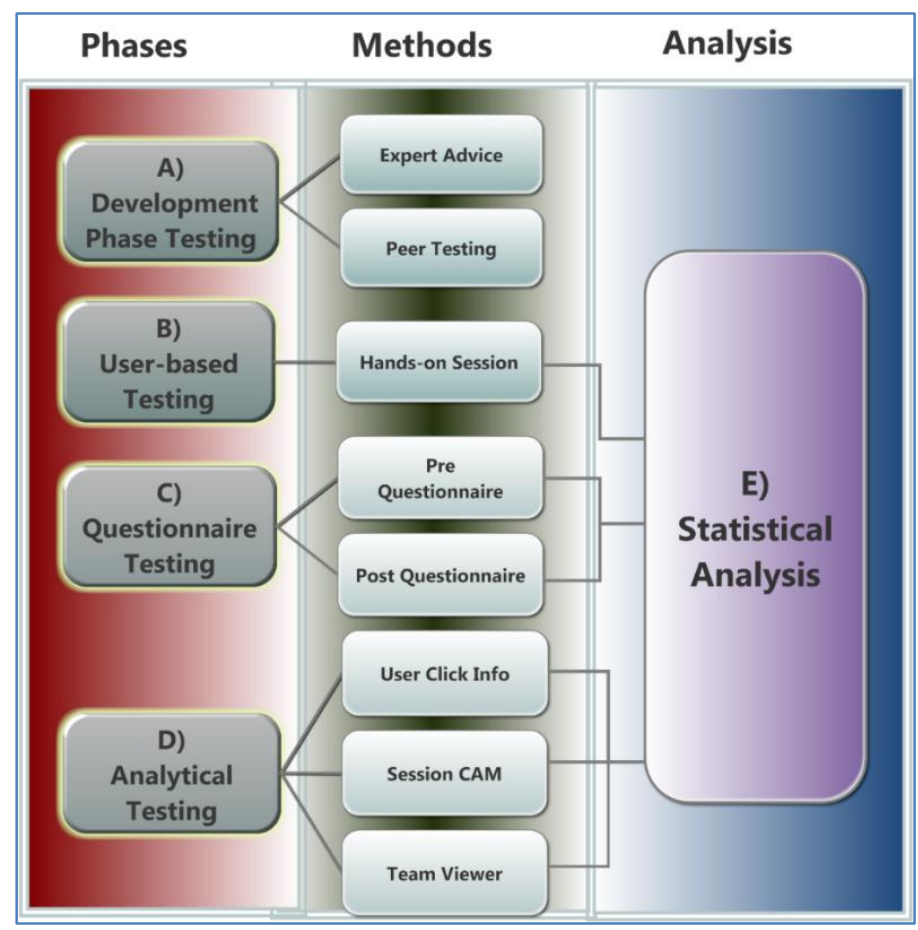

Figure 5.1: Usability Evaluation Flow Diagram for the Assessment of a Co-PPGIS Prototype Application 
Figure 5.1 also shows the sequence of steps adopted during the usability evaluation for a Co-PPGIS prototype application that are used in order to measure whether or not the prototype passes the test based on the selected evaluation criteria. These five steps are briefly explained below:

\section{Development/Design Phase Testing}

This testing approach is usually adopted by each software developer or designer during the Software-Development Life Cycle (SDLC). It is also referred to as an inspection and heuristic evaluation approach in which a cognitive walk-through is performed by examining different functionalities of the developed system. This helps the software developer to determine the usability of a product. The team comprising the system developer and Quality Assurance (QA), is involved in in performing this task; whereas the role of the end user is limited to inspection (Banati et al. 2006, Meng and Malczewski 2009). According to Matera et al. (2005), such technique's assessors examine usabilityrelated aspects of an application, trying to detect violations of established usability principles and then provide feedback to developers about possible design improvements. As shown in Figure 5.1, this method is used as a preusability evaluation during the development and implementation of the Co-PPGIS; therefore, its outcome was not used at the time of performing statistical analysis.

\section{Questionnaire Testing}

Questionnaires are used to analyze the opinion of the users. A preliminary questionnaire and a post-test questionnaire are designed for this purpose.

\section{Preliminary Questionnaire}

A preliminary questionnaire contains queries related to the demographic and socio-economic characteristics of the proposed users. Queries are further designed to ascertain the interaction between the user and the prototype system. The key significance is information pertaining to a user's knowledge and work experience with Web GIS applications, participatory planning, educational and previous web surfing. This information makes it possible to interpret the respective responses and reactions from different participants, as well as helps to set limits to the complexity of the prototype application (Zhao 2007).

The data about the level of participant education was collected using a scale ranging from 1 to $5(1=$ high school diploma, 2 = college diploma, $3=$ university degree, $4=$ Master's Degree and $5=$ Ph.D. degree). Gender, previous experience with GIS and the user's participatory planning experience are coded in a binary form $(1=$ male, $0=$ female; $1=$ user has previous GIS experience, $0=$ not used GIS; $1=$ user has participatory planning experience, $0=$ user with no participatory planning experience). 


\section{Post/Secondary Questionnaire}

An effective usability process should ideally combine the questionnaire and user-based testing approaches. At the end of the online participation period (walk-through of system functionalities), a second questionnaire was designed to gauge the participants' opinions about the ease of use, the clarity of functions, and the design and layout of prototype interfaces.

\section{User-based Testing}

Similar to other testing methodologies, the main aim of user-based testing is to identify the deficiencies in the prototype in order to improve the quality of the concerned system. User-based testing approaches entail data gathering during an active session. The objective is to find the users' mistakes (rate of errors), time taken to become familiar with the prototype, retention over time, and overall performance (measuring speed of performance) while using the system (Butler 1996, Shneiderman 1998, Zhao 2007). Prior to the commencement of the session, the author explained the purpose and expectations of the usability test. All participants were exposed to the same videobased presentation ${ }^{12}$ regarding the prototype system's purposes and functionalities.

\section{$\underline{\text { Task-Based Scenarios }}$}

The user-based test demonstrates how the users accomplish a task and reflects whether the Web interface simplifies the completion of the selected scenario. Usability tasks should be selected to be as representative as possible and to deliver a reasonable exposure of the most significant parts of the Web interface and the system functions (Nielsen 1993). Several task-based scenarios are prepared for the users to walk-through using the Co-PPGIS prototype Web interface. Samples of some of the example tasks ${ }^{13}$ are described as follows (similar tasks were prepared for the participants during the mock case study scenario, as discussed later in Section 5.3):

Example Task 1: This task explores how the Co-PPGIS prototype facilitates a map-based discussion amongst participants during planning and management activities. The discussion pertains to identifying a suitable site. For example, a new student center near Ryerson University, Toronto was proposed. This task hopes to measure the system's efficiency with reference to the number of annotations/arguments posted by different participants in specific period of time.

\footnotetext{
12 One of the CSPE-based map-sharing component functionality is demonstrated to the participants using video-based tutorial, which can be viewed online at: http://coppgis.com/video

${ }^{13}$ Video-based presentations of example tasks can either be downloaded or viewed online at: http://coppgis.com/tasks/ExampleTask1/, http://coppgis.com/tasks/ExampleTask2/ , and http://coppgis.com/tasks/ExampleTask3/ 
Example Task 2: This task examines how Co-PPGIS facilitates the identification of suitable sites for parking in the Dundas and Yong Street intersection in downtown Toronto, Canada. It proposes to measure effectiveness with reference to the frequency of functions that are used to perform the task.

Example Task 3: This task may be used to explore particular incident place marking (e.g., new amendment area in the York regions at Markham, Ontario, Canada). Participants were asked to add a WMS layer related to the new plan of a municipality for discussion. It is required one to draw out a proposed university area in that particular place and add one's comments. The goal is to measure 'usefulness' and 'ease of use' with reference to the occurrence of functions in a specific time frame.

After the completion of the tasks, the results/outcomes were calculated from the number of selected evaluation aspects such as time, accuracy, overall success, and satisfaction measures. Results of the performance perspective (time, accuracy, and success) were considered more important than satisfaction in order to test the prototype against measurable usability goals. For example, in some cases, if testers gave the prototype interface low ratings, the interface needed to be fixed. If testers gave the prototype interface high ratings, a true picture may not have been captured. In usability testing, testers often give high satisfaction ratings even when they have faced serious performance problems. They may blame themselves for the problems. They may not want to hurt the feelings of a prototype developer. They may be polite rather than saying what they really think. Participants' Website tracking, moves and other statistics are observed, recorded and logged using Google analytic, Hit sniffer, Session cam and TeamViewer. These analytical methods are discussed briefly in next section.

\section{Analytical Evaluation and Statistical Testing}

Participant feedback is further evaluated with software tools (e.g., user's screen capturing, video session recording, mouse eye tracking, audio recording and camera-based monitoring techniques). Analytical evaluation was another method adopted during the usability evaluation of Co-PPGIS, which provides the real-time statistics of the user interaction with the system. Hit Sniffer/Google Analytics software-based Website user tracking and other real-time monitoring tools, such as TeamViewer, Clicktale, and Userfly, make it possible to collect detailed and useful data about the actual usage of the prototype and its components. For instance, to assess the degree of public participation on the online Co-PPGIS map-sharing interfaces every move of the user needs to be recorded. For analyzing and understanding how users perform tasks with the computer; the complete actions and moves of the participants can be captured in the interactive sessions by Session Cam and TeamViewer. It tracks actions, such as mouse movements, scrolling a page, or filling out a form, in a specific order. Events, such as opening a website, logging into the system and clicking a button, can easily be stored in a database. Website monitoring tools are useful and helpful in measuring the effectiveness and usability of website performance. These tools are highly valuable for evaluating the 
usability of Co-PPGIS and PPGIS applications. Moreover, by using these tools, evaluators can watch users stream live and instantly in order to see what is happening on the prototype website. The discussion about the statistical testing phase is not covered in this section. It is described in the mock-up case study in Section 5.3.

\subsection{Generating Usability Matrix}

The two methods, questionnaire and analytical testing, are also useful in the development of a design's performance measurement matrix (measuring criteria matrix) for prototype application.

Table 5.1: Specification of Usability Measuring Criteria Matrix

\begin{tabular}{|l|l|l|}
\hline \multicolumn{1}{|c|}{$\begin{array}{c}\text { Usability } \\
\text { Attribute }\end{array}$} & \multicolumn{1}{c|}{$\begin{array}{c}\text { Measuring } \\
\text { Technique }\end{array}$} & \multicolumn{1}{c|}{ Value to be Measured } \\
\hline Intended users & $\begin{array}{l}\text { Pre Questionnaire/ } \\
\text { Hit Sniffer }\end{array}$ & No. of Questionnaire/Hit Sniffer \\
\hline Usefulness & Post questionnaire & Average score (Q1-Q3) \\
\hline Ease of use & Post Questionnaire & Average score (Q7-Q10) \\
\hline Efficiency & Observation & \# of posted arguments/ Hit Sniffer \\
\hline Initial Cost & Absent & Open Source \\
\hline Interactivity/HCI & Post Questionnaire & Average score (Q13-Q17) \\
\hline
\end{tabular}

Table 5.1 describes the techniques used for measuring the specific usability attributes (for questionnaires see Appendix 1). In it, measuring techniques (i.e., pre-questionnaire, Hit Sniffer and/or Google Analytics) are used to find the intended users as a usability attribute with their demographics (i.e., age, education, gender and geographic location) for evaluating the application usability. Similarly, usability attributes (i.e., usefulness, ease of use and interactivity) can be measured from the post-test questionnaire and/or Website monitoring software-based observation (TeamViewer, SessionCam, and UserFly).

Table 5.1 adopted several measures from previous research, including Meng and Malczewski (2009), Rinner (2007), Zhao (2007) and Nielsen (1993, 2000). Usability aspects listed in Table 5.1 were used during the mock case study scenario discussed in Section 5.3. Furthermore, a generic list of usability measuring criteria in relation to the social and practical adoption of the Co-PPGIS prototype interfaces are generated and described in the Tables I and II (see Appendix 7). The ultimate goal of generating the usability matrices and measuring benchmarks of usability is to evaluate synchronous and asynchronous map-sharing mechanisms in order to enhance public participation and provide a user-friendly prototype that includes accurate and interactive interfaces. In this way, a participant can 
communicate effectively and get real-time responses from other participants during a participatory planning related discussion.

\subsection{Case Study: Evaluating the Usability of Co-PPGIS Map-sharing System - As a Participatory Municipal Planning Support Tool}

This section presents the usability in detail. The participants were invited to join the discussion in order to identify planning and development-related concerns of the urban expansion in the York Region. Twenty-five university students were selected, and an interactive work environment was provided to them to introduce the participatory map-sharing mechanism. Their behaviour and response were observed and recorded using software and questionnaire. This data was tabulated and statistically analyzed, proving the system's efficiency, ease of use, understanding and level of user satisfaction. The objective of this study was to test the usability of the prototype by providing a mock case study relevant to municipal planning in the York Region, Canada under its ministry of planning and development.

The planning department of the Regional Municipality of York has selected three potential sites for amendments in the town of East Gwillimbury, Markham and the City of Vaughn in York Region according to its Regional Official Plans of Amendments (ROPA). Hard copy maps related to the ROPA were acquired from the municipality site. Spatial data related to amendments were digitized and prepared using Google Earth in shape file formats, which is loaded as a WMS layer into GeoServer to display as a vector data layer on a map-sharing interface (see Google map in Figure 5.2). In addition, this spatial data was used during the usability evaluation of the Co-PPGIS map-sharing Web interfaces (as described in Section 5.3.3).

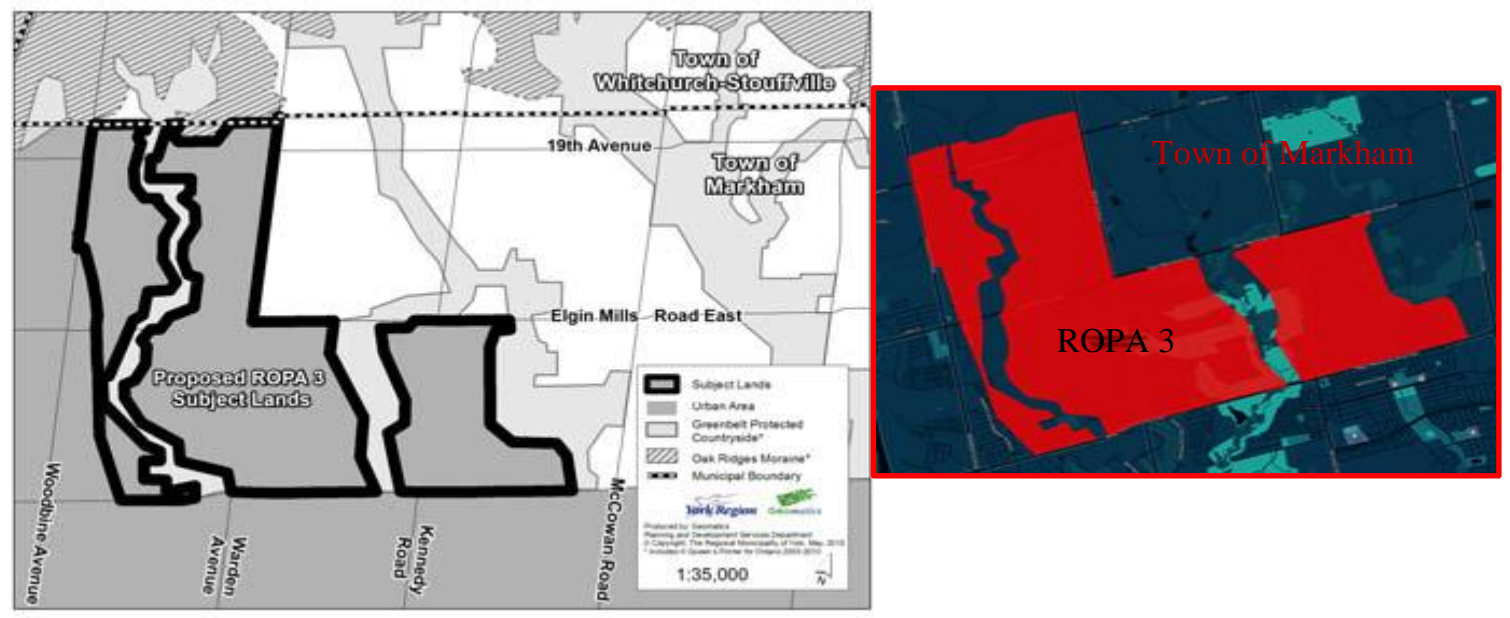

Figure 5.2: Hard Copy Map (on Left) and Google Map (on Right) (Source of Hard Copy Map: http://www.york.ca) 


\subsubsection{Selected Co-PPGIS Prototype Web Interfaces for Scenario-based Testing}

The Co-PPGIS map-sharing mechanism supported synchronous and asynchronous public participation approaches that are selected for the scenario based testing, which are discussed briefly as follows:

Co-PPGIS Synchronous Map-sharing Approach: The Co-PPGIS prototype with a synchronous map-sharing approach can be accessed using any typical Web browser. The main interface of the Co-PPGIS prototype with synchronous map-sharing environment is shown in Figure 5.3.

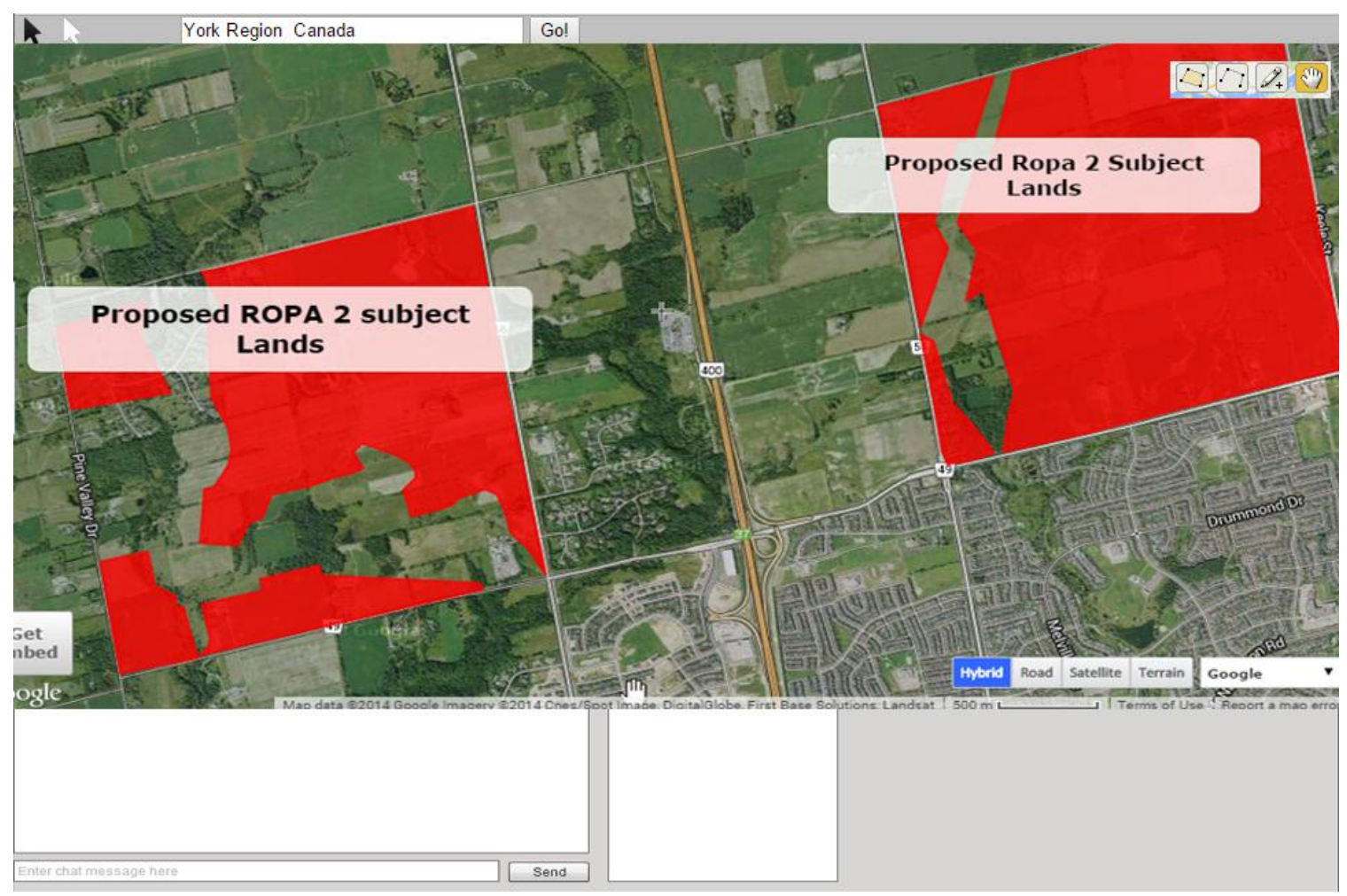

Figure 5.3: Co-PPGIS Map-sharing Interface Overlaid the York Region Proposed Amendment Plan (ROPA 2) in the City of Vaughan. Web URL to Access Synchronous Co-PPGIS Map-sharing Web Interface: http://coppgis.com/geomeet1

Co-PPGIS Asynchronous Map-sharing Prototype: The prototype's Web blog interface provides the participants with the ability to contribute to and/or be involved in using the asynchronous map-sharing approach during planning and development workflows. 
The Co-PPGIS prototype's blog interface with the York Regional Municipality (http://www.york.ca) amendment plan information (the Google map and municipality vector data) is shown in Figure 5.4.

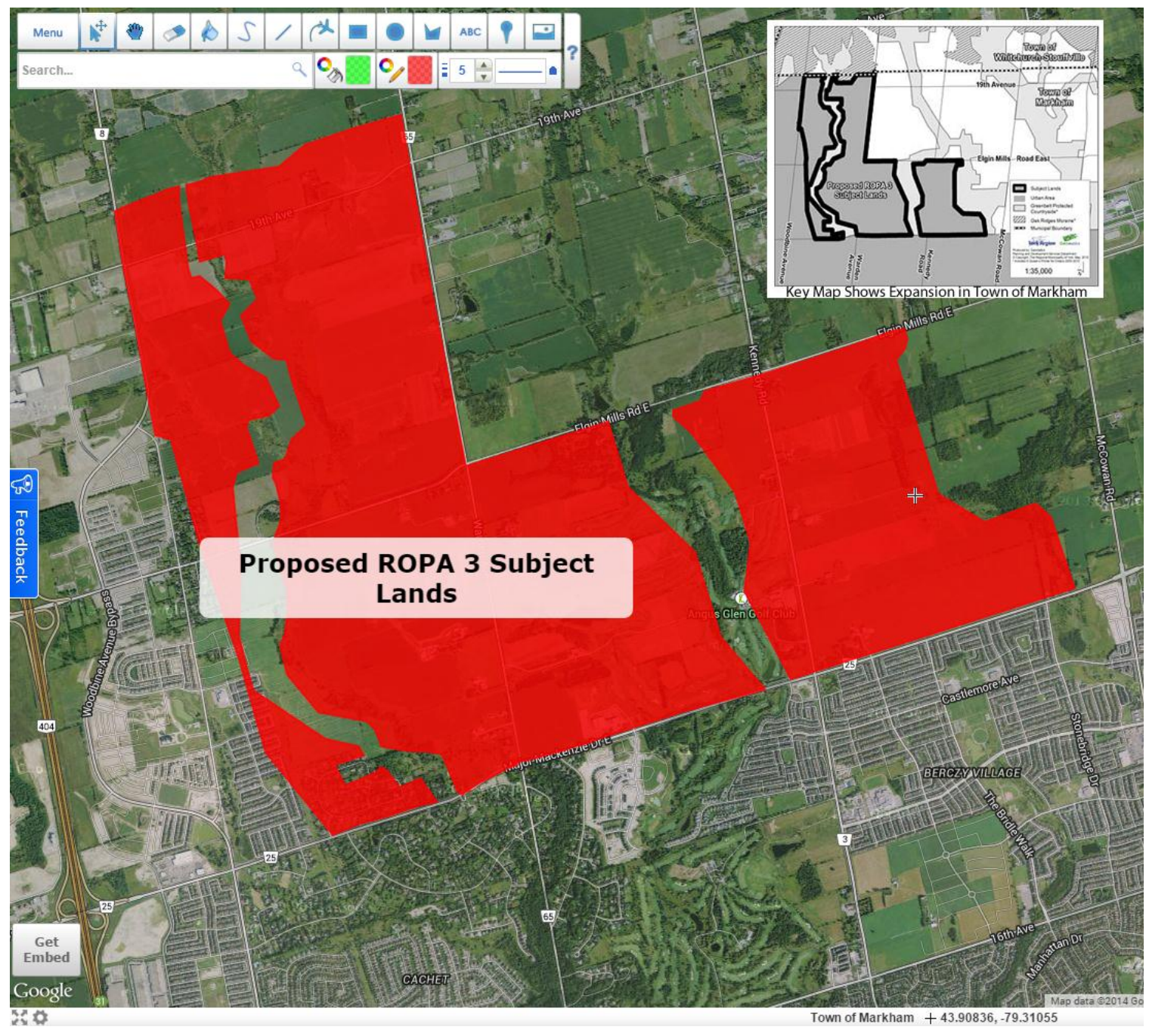

Figure 5.4: Spatial Data of York Region (Town Markham Amendment Plan) Overlaid on the Google Map (Base Layer) Using Co-PPGIS Component Discussed in Section 4.2.1, URL to Access CAPE Blog: http://coppgis.com/wolpgis-f/?p=42

\subsubsection{Usability Evaluation Criteria of Co-PPGIS}

The perspective of the case study is to assess and evaluate a Co-PPGIS map-sharing mechanism that is developed for responding to various municipal planning and management situations. This system is designed in such a way that participants/attendees working in different departments associated with planning and management workflows shall participate and play their respective roles. 
The criterion factors may include effortless use of the real-time collaboration, competence, the initial and monitoring cost of the prototype, and intercommunication or Human-Computer Interaction (HCI) of the prototype in the long run. In brief, the usability criteria have to be carefully selected. Another critical factor is to include the user's requirements and needs for the development of an application. In this mock case study, the following aspects (already discussed in Table 5.1 and in Appendix 7) are selected:

- Usefulness/ Effortless Use: The prototype can be effortlessly utilized by stakeholders for planning, sharing maps and other spatial information in real-time. This was monitored by the number of clicks done to perform a single task during the testing period.

- Efficiency/ Competence/ Understanding: Effective participation deals with the application's functionality, observation of user's performance and experience of tasks. The public participation requirements should be addressed in a more efficient manner. The user's performance was judged by the efficient completion of the given assignments within the allotted time frame.

- Initial Cost/ System Cost: The system cost was evaluated in terms of the design and development of the prototype. In Co-PPGIS, the prototype is recommended to be developed using open-source software and GIS technologies; and therefore, renders no charges.

- Interactivity/ User Contentment/Ease-of-Use: Co-PPGIS will provide a user friendly, efficient, accurate and interactive interface, so that a participant can quickly communicate and get a response from other participants about their inquiry. No doubt, a quicker response time improves interactivity and satisfaction among users.

\subsubsection{Usability Evaluation Methodology for Co-PPGIS Map-sharing Interface}

The following four methods have been selected from usability evaluation framework and applied during the usability evaluation of the Co-PPGIS map-sharing mechanism.
A. User-based testing
B. Questionnaire-based testing
C. Analytical Testing
D. Statistical Analysis 
Figure 5.5 describes the framework of the usability evaluation that was carried out to judge the user's interaction with this Co-PPGIS prototype.

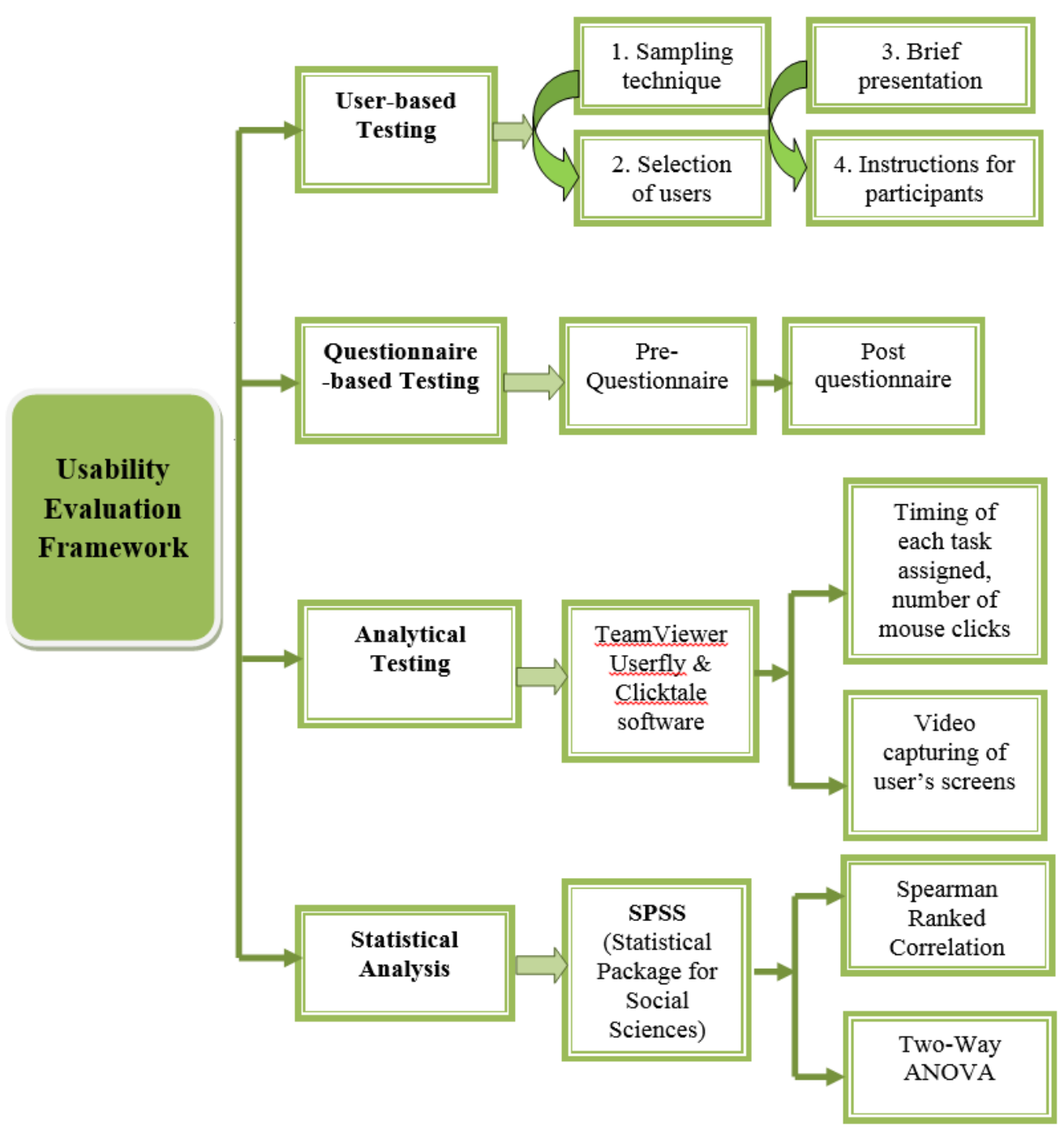

Figure 5.5: Usability Evaluation Framework Adopted for Co-PPGIS Prototype

The user's movements regarding the work assigned were recorded through e-monitoring software. The output data collected with the help of TeamViewer and Session Cam software, along with feedback forms, were entered into the statistical software (SPSS) for further analysis. Each of these stages is discussed in detail in the later sections. 


\section{User-based Testing}

The user-based testing method was employed in this study to determine the shortcomings and to improve the functionality of the prototype. The user testing method employs techniques for collecting data, engaging users from different backgrounds to interact with the system and perform assigned tasks. In this case, usability can be specified in terms of users' performance and satisfaction when interacting with the system (Butler 1996). The following describes the various steps of usability evaluation.

\section{Sampling Technique}

A stratified sampling methodology was adopted in this study for testing and evaluating the Co-PPGIS application map-sharing mechanism. A stratified sample is obtained by independently selecting a separate random sample from each stratum. The target population was divided into five different groups based on enrolled programs in the Ryerson University.

\section{Selection of Users}

The usability testing in which users were directly involved in was conducted in three stages: Pre-test questionnaire, user's monitoring and post-test questionnaire (all three techniques are designed using online Web technologies in order to facilitate the participants). In the first stage, a pre-test questionnaire was given to collect each user's basic characteristics, educational background, and familiarity with GIS and IT applications (see Appendix 1 and 2 or see Web source at http://coppgis.com/feedback). Since this is the mock-up testing scenario, the preference was to invite a multidisciplinary group of participants, who work in science and related professions. Five groups were formed among different categories of students who were selected from Ryerson University. Students were asked to volunteer for the usability testing of the Co-PPGIS prototype application.

The selected groups of university students were as follows:

Group I: Urban Planning

Group II: Environmental Engineering

Group III: Geography/Civil Engineering with GIS background

Group IV: Management

Group V: English Literature

\section{Brief Presentation}

Some university students used their own laptops at their own convenience (classroom, home, student centres) and some students were taken to the library, which was ready with desktop computers equipped with a high-speed 
Internet. Session cam is a built-in plug-in of the Co-PPGIS blog application, which can be used only in an asynchronous map-sharing environment to record each user's move. TeamViewer software was installed on the host and participant computer to monitor and record each user's interaction with the interface. Both software provide a similar kind of monitoring function, but TeamViewer provides a more user-friendly environment that can be used in both a Co-PPGIS synchronous and asynchronous map-sharing environment. Therefore, it was mostly used during the testing and monitoring of each user's moves in order to analyse their interaction with the application interfaces.

At the start of the evaluation, students were requested and required to watch a twenty minute demonstration video (see http://coopgis.com/video) on the usage and background of the Co-PPGIS map-sharing application. The purpose was to introduce and highlight the ways of public participation, collaborative decision-making and other important functional elements. Furthermore, this video-based tutorial was intended to increase the performance in achieving tasks, especially for participants who have less knowledge of GIS and IT. It also was beneficial in identifying the performance criteria indicators, discussed later in the Usability Indicators section.

\section{Tasks / Instructions for Participants}

Usability evaluation tasks or instructions for participants were designed to help them understand the idea of decision-making during planning and management situations. The usability evaluation test showed how easily the public (Ryerson University students in this case) can participate in the municipal planning process.

The evaluation tasks ${ }^{14} /$ instructions are briefly explained below:

1. After filling out the online pre-test questionnaire form, the users were asked to log in the Co-PPGIS Web interface by entering their name. After login, they were asked to search for a location on the York Region's amendment plans or any place related to the study area. This task was performed by accessing two CoPPGIS application components (see Figure 5.3 and 5.4) that are designed to support the synchronous and asynchronous map-sharing mechanism.

2. They were then asked to use the whiteboard tools to draw on the map of the selected region. This task includes navigation, marking a place in context of planning, and commenting on a shared map view.

3. Users were asked to use the chat panel, which opens in a separate window.

4. Users were then provided with different photographs, videos and documents downloaded from York Region's website, and asked to upload them, so the fellow users who can see and suggest a solution for that particular problem.

\footnotetext{
${ }^{14}$ Video-based presentation of these tasks (Task 1 to Task 5) can either be downloaded or viewed online at URL: http://coppgis.com/tasks
} 
5. Users were asked to mark the new area for a health facility in the city of Vaughan of York Regions on the map. Participants were asked to enable a WMS layer related to the new amendment plan of a municipality in the York Region for discussion. This task required them to draw out a polygon in order to indicate a proposed health facility area and add their comments.

In the end, users were redirected to a web page to complete an online feedback form (post-test questionnaire at http://coppgis.com/feedback1

\section{Questionnaire-based Testing}

At the beginning of the evaluation session, users were asked to fill-out an online pre-test questionnaire (see Appendix 1) comprised of socio-demographic and technical knowledge indicators. A pre-test questionnaire was prepared to understand the influence of usability indicators such as education, gender, major area of specialization, and IT/GIS knowledge with the Co-PPGIS application interface. At the end of the Web-based discussion session, users were asked to fill-out the online post-test questionnaire (see Appendix 2). In this questionnaire, indicators, such as effortless use, competence, innovative, user contentment, learnability, efficiency, content relevance, participatory planning, and communication ways for using the Co-PPGIS prototype, were incorporated. In brief, a post-test questionnaire was prepared and developed to identify the user's contentment with the Co-PPGIS interface.

\section{Analytical Testing}

As mentioned earlier, the second part of the evaluation was the user's interaction with the Co-PPGIS system. The participants were asked to interact with Co-PPGIS Web application. This Web-based virtual discussion was monitored and judged with the help of TeamViewer, Clicktale, Userfly, and Session Cam software. The Session Cam, Userfly, and clicktale capture every mouse click (user click info), scroll and keystroke that a user made to perform a task, and then sends this information back to the servers in a highly compressed package. These software plugins were helpful, but could only be used in CAPE-based prototype interface usability testing, due to its less support for real-time CSPE-based application.

On the other hand, TeamViewer can be used in both CAPE and CSPE-based prototype interfaces. Moreover, the TeamViewer software captures user's screens in a video form and records mouse movements and time to finish the task of every participants. This technique is convincing, but little inflexible in relation to the counts of mouse clicks, as moderator requires to count each mouse click manually by watching the video of every participants. Also, using TeamViewer recording tool, it is easier for the moderator to track and analyze the participant's movements and their level of understanding in using the Co-PPGIS Web interfaces. 


\section{$\underline{\text { Usability Indicators }}$}

Usability Indicators were selected by keeping in mind the nature and number of tasks assigned to the participants. Figure 5.6 presents an overview of the usability indicators that were chosen to assess each participant's ease in interaction with the Co-PPGIS prototype interfaces.
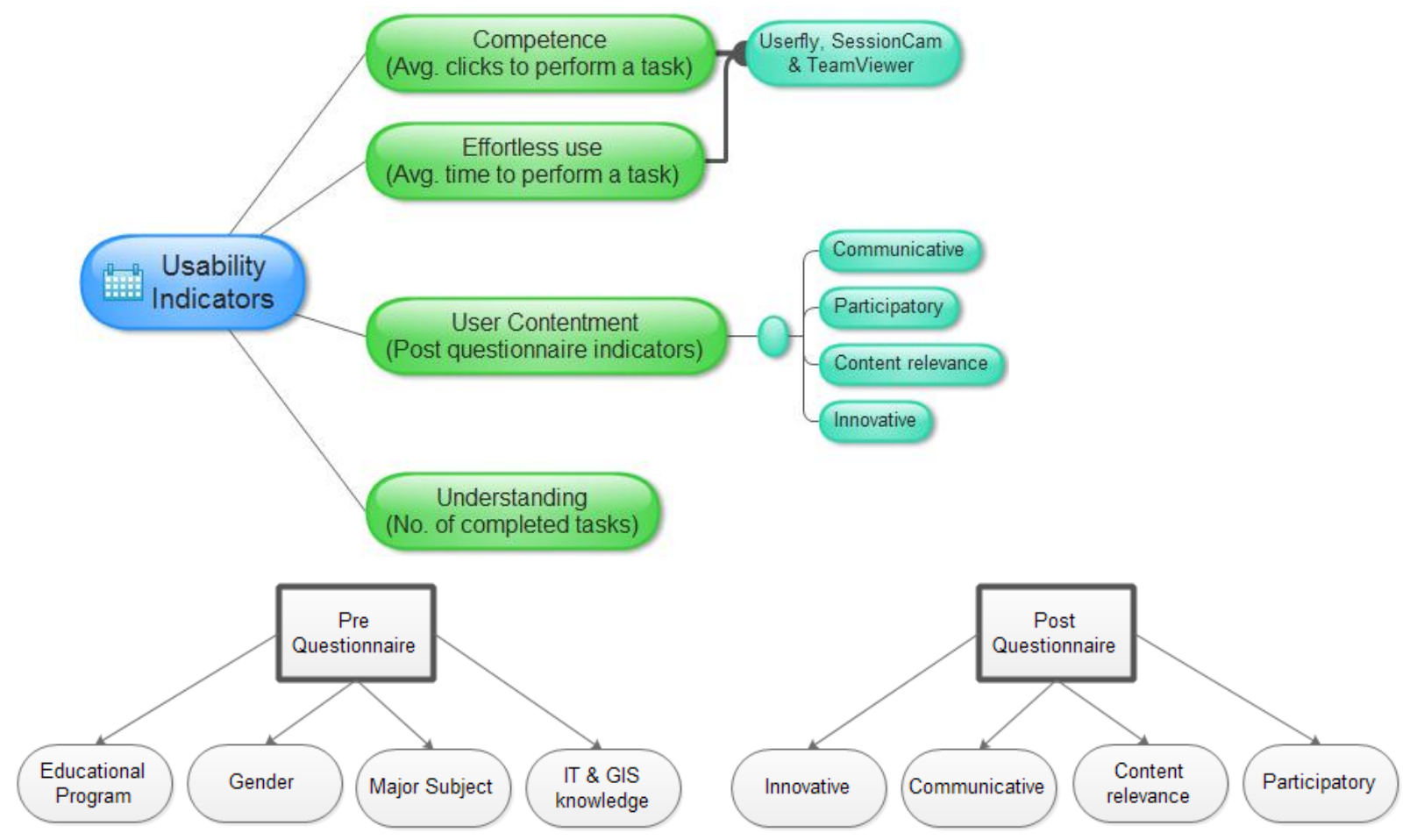

Figure 5.6: Measurement Tools Used in This Study to Determine Usability Indicators

As each task required a certain number of clicks, and time needed to complete, performance criteria were established based on time limits and number of clicks to complete a task. Pre-testing tasks were given to the participants by selecting a random number of students from each group in order to compute the required number of clicks and time to perform a single task. Preferably, the time limit was set according to the nature and complexity of the given task. A total of thirty-forty minutes were given to understand the instructions and perform all five assignments, which may require unequal time of duration. In addition, each task was pre-performed by the moderator ${ }^{15}$ in order to provide a benchmark for number of clicks and time limits, with some flexibility for non-IT and non-GIS

\footnotetext{
${ }^{15}$ Video-based tutorial in relation to each task can either be downloaded or watched online at URL: http://coppigs.com/video
} 
professionals. The time taken for each participant to finish an assignment was recorded with the help of TeamViewer and Session Cam software. Performance criteria were also established as:

○ Ideal click by a user to perform each task has direct relationship to the complexity of the task (e.g., a more complex task requires more clicks).

- Similarly, the ideal time to finish each task depends upon the nature of the task (e.g., a lengthy task requires more time).

Nielsen (1993) suggested that efficiency, learnability, fewer errors, understanding and contentment are the main components of usability. The usability was evaluated by recording the number of clicks for a given task (calculating user's competence), number of tasks performed within the given time (measuring user's understanding of the system), time to perform a single given task (effortless use of the Web-application) and contentment (measured through these variables, i.e. relevance of content, innovative, communicative and participatory).

Since usability is primarily a user-oriented concept, the term user contentment has been included. This term implies that the user was able to satisfactorily perform the task which he/she wanted to perform. During the study, it was observed that a few users were sitting idle and just clicking the mouse. The reason for this behaviour is that perhaps they "got stuck" causing them not to be able to complete the given assignment in the given time period.

Statistical analysis and results based on these usability indicators are described in the next section.

\section{$\underline{\text { Statistical Analysis }}$}

Statistical analysis was performed on the user performance data, which was collected from Web- based pre-post activity questionnaires and analytical software. All data was fed into the Statistical Package for Social Sciences (SPSS) for statistical analysis ${ }^{16}$. The results are discussed in this section.

Moreover, the statistical analysis section covers the following components:

a) Participant's Characteristics

b) Usability Indicators

c) Correlation Interpretation

d) ANOVA Interpretation

${ }^{16}$ Some statistical results can be viewed and compared online using feedback component (through admin panel, which was developed as a part of this component) using these online URL: http://coppgis.com/feedback/admin and/or http://coppgis.com/feedback1/admin, provide username $=$ admin password $=1234$. 
Participant's Characteristics: The gender ratio of male to female of the selected users was 9:16. The percentage of graduate (undergraduate) and post-graduate (masters) students was about the same (48\%), while a single doctorate student (4\%) was presented in the sample population (see Appendix 3). Judging the IT and GIS skills of the students from the pre-questionnaire, it was observed that $60 \%$ had a good level of computer (IT) knowledge while $36 \%$ had beginner level skills in GIS. The majority of the respondents were familiar with computer (IT) knowledge, having Internet navigating proficiency and basic GIS skills. Four of the 25 participants had expert-level computer skills and were familiar with web applications and the basics of using GIS as well. Five of the six respondents from the GIS program had expert-level web surfing and usage skills. As for educational level, $90 \%$ of the group were postgraduate degree holders.

The graphs presented in Figure 5.7 depict the users' characteristics in percentages. IT and GIS knowledge area marked on a scale of $0-4$, with $0=$ nil, $1=$ beginner, $2=$ moderate, $3=$ good, and $4=$ very good levels.
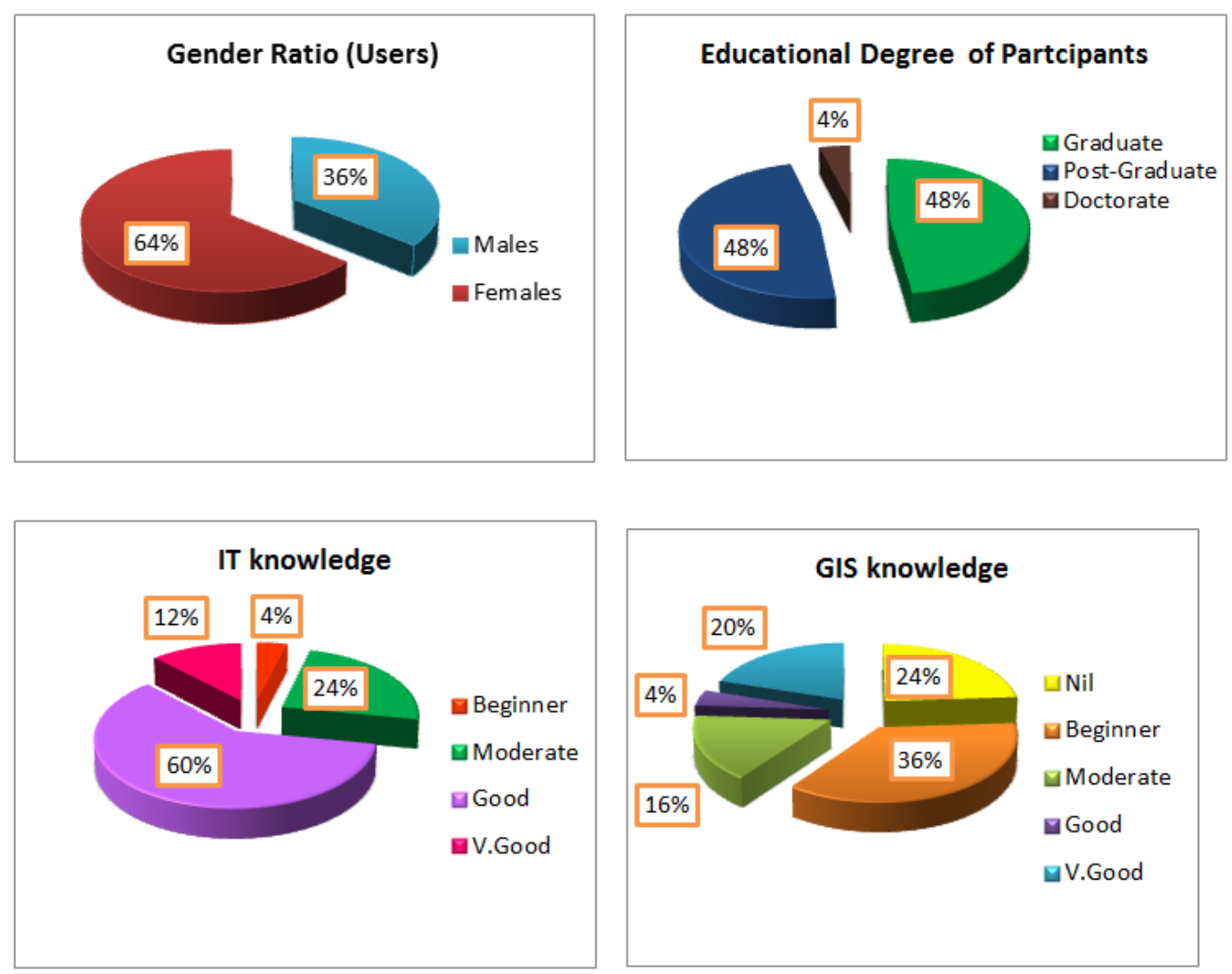

Figure 5.7: Graphical Analysis of User Characteristics 
Usability Indicators: The results collected from the software and feedback (post-test questionnaire) at the end of the testing session are graphically presented in Figure 5.8:

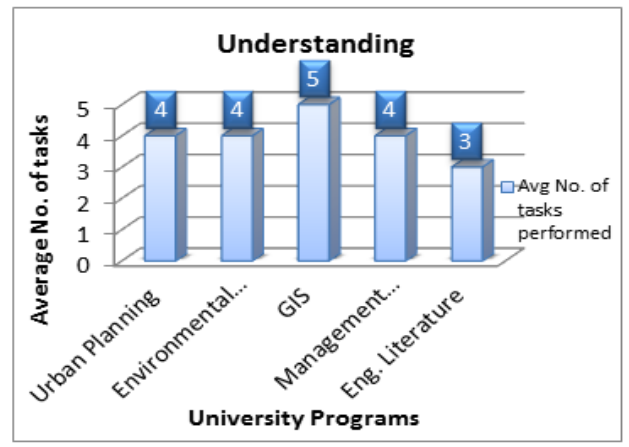

Graph 1

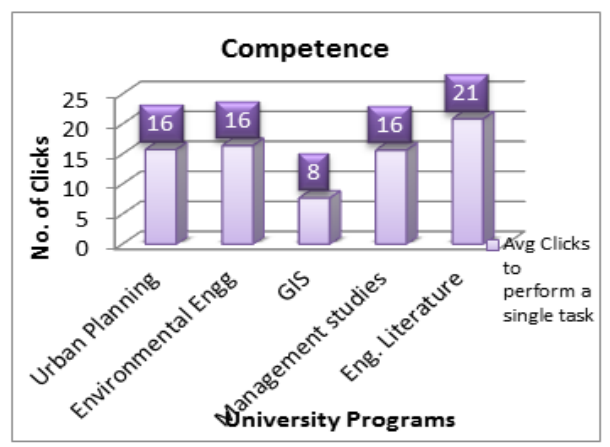

Graph 2

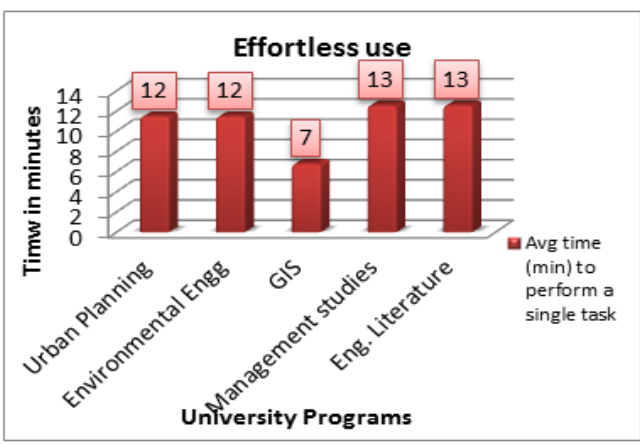

Graph 3

Figure 5.8: Graphical Analysis of Usability Indicators Derived from User Performance

Graph 1 of Figure 5.8 represents the understanding of the Co-PPGIS prototype interfaces by the participants based on tasks performed. Each group, consisting of five participants, performed a varied number of tasks from which an average was taken. Graph 2 depicts the competence, which is measured by the average number of clicks taken by each group to perform a single task. Since effortless use was determined by the average amount of time taken to complete a single task by the group members, Graph 3 represents time in minutes to complete a task showing ease of use for the user.

These results demonstrate that the GIS group of university students had an edge over the other groups as they had better GIS knowledge and computer skills. The graphs displayed in Figure 5.8 clearly indicate that the GIS group took less time to complete the given tasks as compared with the rest of the students. Urban planning, environmental 
engineering and management studies groups also performed well in the tasks. The English literature group was a little behind the others and took relatively more time and clicks to understand and accomplish the tasks.

Correlation Interpretation (Spearman Rank Correlation): Spearman's correlation helps to identify whether the correlation is positive or negative. As with the simple correlation coefficient, Spearman's correlation coefficient also obeys $-1 \leqslant r \leqslant 1$ where $r=1$ corresponds to a perfect positive correlation and $r=-1$ to a perfect negative correlation. The result should always be between +1 and -1 . Spearman's rank correlation works by converting each variable into ranks. Once the two variables are converted into ranks, it is easy to perform a correlation analysis on the ranks. Its values are calculated using SPSS, which provide output up to three decimal places, in order to measure results (i.e., no of tasks performed, average clicks, and average time to perform a task) with more precision and sensitivity and to find significant differences.

Details of the results of Table 5.2 are discussed in next section against each usability indicator and user characteristic. The data collected through usability software (TeamViewer and User click info), and the post-test questionnaire were given ranks ranging on a scale of 1 to 5. Table 5.2 (values are given to 3 decimal places) displays positive and negative correlations between the usability indicators (obtained through user's screen monitoring) and user's characteristic variables (obtained through questionnaires).

Table 5.2: Spearman Correlation between Usability Indicators and User Characteristics

\begin{tabular}{|c|l|l|l|l|}
\hline \multirow{2}{*}{ Sr no. } & \multicolumn{1}{|c|}{ Usability Indicators } & \multicolumn{3}{c|}{ User Characteristics } \\
\cline { 3 - 5 } & \multicolumn{1}{|c|}{$\begin{array}{c}\text { GIS } \\
\text { Knowledge }\end{array}$} & $\begin{array}{c}\text { IT } \\
\text { knowledge }\end{array}$ & Education \\
\hline $\mathbf{1}$ & $\begin{array}{l}\text { Competence: } \\
\text { Avg Clicks to perform a single } \\
\text { task }\end{array}$ & -0.675 & -0.587 & 0.256 \\
\hline $\mathbf{2}$ & $\begin{array}{l}\text { Understanding: } \\
\text { No of tasks performed }\end{array}$ & 0.714 & 0.626 & -0.143 \\
\hline $\mathbf{3}$ & $\begin{array}{l}\text { Ease of Use: } \\
\text { Avg time (min) to perform a } \\
\text { single task }\end{array}$ & -0.695 & -0.541 & -0.054 \\
\hline
\end{tabular}

(See Appendix 4 to find detail of these calculated values) 


\section{Interpretation of Usability Indicators Correlated with User Characteristics of Table 5.2:}

In correlation, +-0.5 or greater shows a strong correlation. Values which are near to 0 show a weak correlation.

\section{Competence}

The correlation results showed that there was a negative relationship between GIS and competence (-0.675) and IT knowledge of the users and competence (-0.587). These values indicate that there is an inverse relationship between the two indicators, meaning that the group with good GIS and computer knowledge should have less number of clicks to accomplish a single assigned task. The correlation between education of users and average clicks to perform a task is weakly positive (0.256), meaning that even an educated user can get confused regarding the application usage and randomly start clicking the wrong tabs on the site.

\section{$\underline{\text { Understanding }}$}

The correlation between GIS knowledge and understanding and between IT knowledge and understanding is 0.714 and 0.626 , respectively, confirming that users that are more knowledgeable may perform more tasks in certain time. On the contrary, a weakly negative relationship (-0.143) exists between educational level and number of tasks performed, indicating that the less educated user can still perform the given Web-application usability tasks (see Figure 5.9).
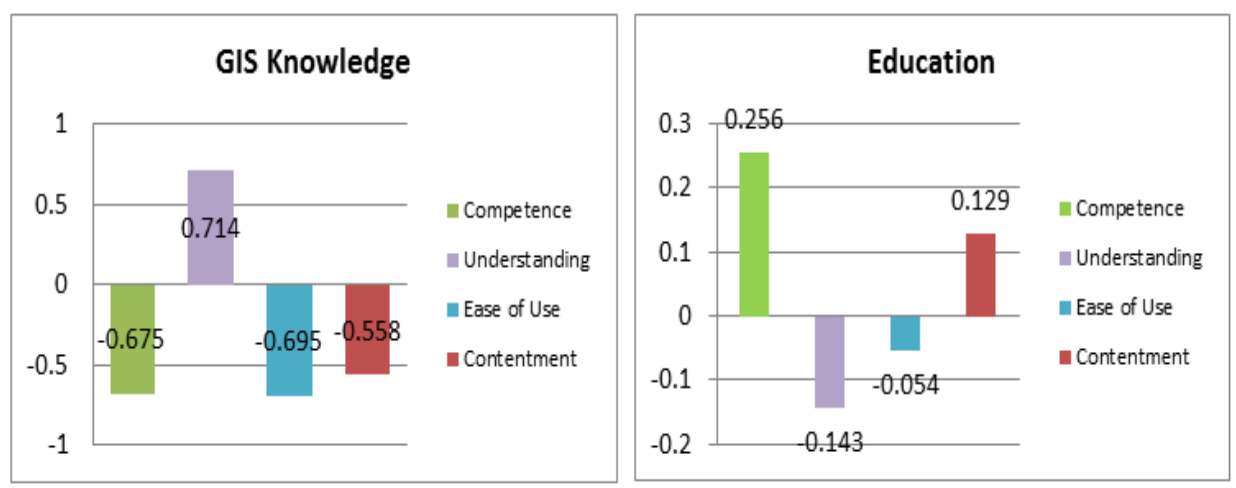

Figure 5.9: Graphical Analysis of Correlation Results of User Characteristics with Usability Indicators

\section{Effortless / Ease of Use}

In the case of time consumed by the users on the tasks, a strong negative correlation can be observed between ease of use and three user characteristics: GIS knowledge (-0.695), IT (-0.541), and education (-0.054), showing that the 
users having a good level of computer know-how tend to finish the given tasks in less time. Correlation of IT knowledge with usability indicators are illustrated in Figure 5.10.

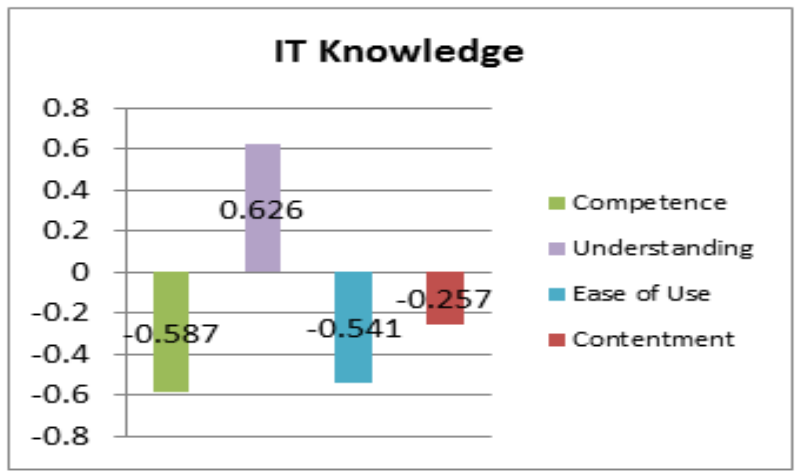

Figure 5.10: Correlation (Graph) of IT Knowledge with Usability Indicators

\section{User Contentment}

Table 5.3 presents the results of correlation analysis between user contentment indicators and the four indicators in the post-test questionnaire (innovative, relevance of content, communicative, and participatory).

Table 5.3: Spearman Rank Correlation between User Characteristics and Contentment Indicators

\begin{tabular}{|l|l|l|l|}
\hline $\begin{array}{l}\text { User Contentment } \\
\text { Indicators }\end{array}$ & \multicolumn{1}{|c|}{ Gender } & \multicolumn{1}{|c|}{$\begin{array}{c}\text { Group of } \\
\text { users }\end{array}$} & Education \\
\hline Innovative & 0.178 & 0.241 & 0.335 \\
\hline Content Relevance & -0.368 & 0.445 & 0.006 \\
\hline Communicative & -0.326 & 0.083 & -0.187 \\
\hline Participatory & 0.393 & -0.417 & -0.127 \\
\hline
\end{tabular}

Positive correlation of $0.178,0.241$, and 0.241 is observed between "Innovative" indicator and gender, group of users, and education, respectively, showing that all the participants found the web-based application somehow innovative. Similarly, there is a weak positive correlation (0.083) between the group of users and the attribute of communication. A positive correlation exists between the gender (0.393) and the participation attribute, illustrating that they all found the prototype participative. A negative correlation exists between the group of users and the participation attribute (-0.417), illustrating that non-GIS groups do not found the prototype participative. A negative correlation of -0.368 and -0.326 is observed between content relevance and communicative with gender showing that the prototype interface is less content relevance and communicative. In addition, a positive correlation (0.445) is 
observed in group of users with content relevance, which means that group of users found interface more relevant to its content.

Interpretation of Two-Way ANOVA: The reason for doing a Two-way Analysis of Variance (ANOVA) is to see if there is any difference between groups on one variable. The four main usability indicators, total time consumed, total clicks, time spent on each task and total tasks completed, have been studied in relation to group performance by applying a two-way ANOVA. The 2-way ANOVA (Tukey HSD) results presented in Tables 5.4 and 5.5 (see details in Appendix 5) show the comparative ranking of different group performance on 'average clicks to perform a single task' indicator.

Table 5.4: Tukey HSD - Average Clicks to Perform a Single Task (All Groups)

\begin{tabular}{|l|l|l|l|l|}
\hline Sr & Groups & Ranking & Mean & Mean difference w.r.t former level \\
\hline $\mathbf{1}$ & GIS & 1 & 7.640 & n-a \\
\hline $\mathbf{2}$ & Management studies & 2 & 15.680 & 8.04 \\
\hline $\mathbf{3}$ & Urban Planning & 3 & 15.740 & 0.06 \\
\hline $\mathbf{4}$ & Environmental Engineering & 4 & 16.420 & 0.68 \\
\hline $\mathbf{5}$ & English Literature & 5 & 20.860 & 4.44 \\
\hline
\end{tabular}

The group that has the least mean difference performed a single task with minimal clicks. The GIS group is the most efficient group in comparison to the other groups and the Management Studies group, which is second in ranking and has the double mean difference compared with GIS group. It clear that the GIS group has a significantly higher ranking (1) and that the English Literature group with (5) has the lowest ranking in terms of average clicks; thus, showing greater efficiency of the former as compared with the latter group. The GIS group is the most efficient in performance as this group has the required skills to carry out the tasks on time. The difference between the rests of the groups in terms of efficiency is small. The mean difference between Management Studies and Urban Planning is 0.06, and between Urban Planning and Environmental Engineering is 0.680 while it is 4.44 between Environment Engineering and English Literature. The English Literature group comes in last, with a large difference between its mean average and that of the $4^{\text {th }}$ ranking group, Environment Engineering. 
The rest of the non-GIS groups perform relatively well, which shows that system is user-friendly and was understood by non-GIS users as well. Furthermore, relationship between GIS and other groups is shown in Table 5.5.

Table 5.5: Tukey HSD - Average Clicks to Perform a Single Task (GIS vs. Other Groups)

\begin{tabular}{|l|l|l|}
\hline (A) Group & \multicolumn{1}{|c|}{ (B) Groups } & $\begin{array}{l}\text { Mean difference } \\
\text { (A-B) }\end{array}$ \\
\hline GIS & English Literature & -13.220 \\
\hline & Urban Planning & -8.100 \\
\hline & Environmental Engineering & -8.780 \\
\hline & Management Studies & -8.040 \\
\hline
\end{tabular}

From the results in Table 5.5, it can be seen that the mean difference between the highest scoring (GIS) and lowest group (Eng. Lit) is about 13. The mean differences between GIS and other groups are about 8 . This indicates an unfavourable behaviour between GIS and English Literature groups, which means English Literature group has less required skills (with GIS/IT knowledge) to carry out the tasks on specific time.

\subsubsection{Summary}

This chapter starts with a brief discussion related to the proposed usability evaluation methodology for Co-PPGIS. Five methods are discussed followed by a discussion related to the case study (i.e., evaluating the usability of CoPPGIS - as a participatory municipal planning support tool). In the end, statistical analysis along with Spearman and ANOVA correlation-based interpretations are discussed.

Next, Chapter 6 describes conclusions and recommendations for this research study. 


\section{Chapter 6 Conclusions and Recommendations}

This thesis study is an attempt to integrate functionality of CSCW, groupware, OSGIS, and other OSS tools and technologies in order to develop synchronous and asynchronous collaborative map-sharing capabilities to let public participate in the municipal planning and management-related activities. The focus was to develop an understanding of the existing participation practices and PPGIS requirements, and to fit Co-PPGIS with groupware functions into municipal planning processes with the purpose of increasing public participation, supporting real-time collaborative decision-making, increasing municipal project notification and coordination, and promoting collaborative activities. A Co-PPGIS framework and a research prototype have been developed based on the UML-based requirement modeling and evaluated in terms of the usability of the prototype in a mock case study scenario. This chapter summarizes the work done during the thesis study, presents the key research and development outcomes and discusses some future works.

\subsection{Realization of Research Objectives}

The research study was aimed at enhancing synchronous and asynchronous collaboration capabilities into a municipal planning practices and PPGIS environment. More specifically, it examined OOSE methods along with enabling technologies to design a real-time Co-PPGIS, including a conceptual model, an architecture model, and an implementation model to support public participation and collaborative decision-making. To realize these objectives, this research was carried out in the following steps:

\section{Requirements Modeling for Developing a Co-PPGIS Concept}

Preliminary research objective (i.e., requirements modeling of Co-PPGIS) was to study and model the requirements engineering and analysis of a Co-PPGIS concept, which relates to the basic research questions: How can Co-PPGIS complement existing planning and development-related workflows? What can Co-PPGIS do if it has real-time mapsharing capability?, and how can Co-PPGIS provide a virtual meeting solution to increase public participation and collaborative decision-making, which in turn, considerably improve the efficiency of geographically diverse stakeholders and decision-making authorities? The requirements were collected through the literature review, evaluation and comparison of existing PPGIS and closely related research models, review of concepts related to CSCW and groupware system, and usability studies of some existing PPGIS.

\section{To Design a Real-time Co-PPGIS That Can Employ OSS Tools and Groupware Technologies}

The first objective of this research was to design a Co-PPGIS and its use for supporting public participation to accomplish an enhanced collaborative decision-oriented response during municipality management situations. 
Therefore, the research made a significant effort to design a Co-PPGIS model in order to support synchronous and/ or asynchronous participation approaches along with OSS-based CSCW/groupware technologies integration. This helps in refining the issues related to municipal planning, to improve public input in the existing participatory practices, and to enhance current PPGIS frameworks. A Co-PPGIS design concept includes: (a) a conceptual model, (b) a Co-PPGIS meeting infrastructure, (c) a context model, (d) high-level architecture; and (e) data flow model to facilitate the design of the Co-PPGIS (discussed in Chapter 3). To achieve this objective, an OOSE-based methodology was adopted and the existing municipal planning practice was modeled using UML as well as public participation requirements necessary to support Co-PPGIS environment.

\section{To Develop and Implement a Prototype Based on the Co-PPGIS Concept}

This was aimed at fulfilling the second research objective, i.e., an implementation of Co-PPGIS framework to facilitate the development of the collaborative PPGIS. A modular and incremental development approach was adopted for this purpose. This research combined the collaborative GIS, groupware, the Web and other web-based information technologies, into an online virtual meeting environment and a complementary solution to existing public participation practices was developed to enhance public input and better decision-making. The prototype of Co-PPGIS provided a web-based synchronous and asynchronous map-sharing platform that encouraged more people to participate in the planning and development processes. To achieve this objective, the suitable OSS-based enabling technologies were examined, evaluated, and selected for the development and implementation of Co-PPGIS prototype. The prototype components were developed to support two major participatory environments (i.e., CAPE and CSPE). A map-sharing module was developed to support both the participatory environments. This module provided some major widgets services. An expert level of programming skills and a significant amount of work (i.e., a thousand lines of code in MXML, Java, and JavaScript programming languages) was done during this research study in order to develop and implement the required Co-PPGIS map-sharing services.

Furthermore, the selected enabling technologies were studied, integrated, configured and managed, which especially supported the real-time collaborative map-sharing behaviour of the CSPE and CAPE. These technologies were integrated using Red5, JAVA Servlets, and Adobe Blazeds. The research study and the project work related to the implementation of Co-PPGIS have been proven an outstanding learning endeavour for future research work in the relevant areas.

\section{To Evaluate the Usability of Co-PPGIS Prototype Using a Case Study Scenario}

The third objective of this research study was to provide an insight into the usability evaluation of the developed CoPPGIS prototype interfaces. The scope of this evaluation was however limited due to the lack of actual municipal 
projects related data. The evaluation was conducted in five parts: 1) a pre-test questionnaire with queries about the user's background, computers expertise and GIS knowledge; 2) user-based testing, in which different tasks related to planning scenarios were performed by the participants; 3) collection of user performance related data (i.e., total number of clicks, number of tasks completed, and time to perform a single task) using an analytical method with the help of Google Analytics, TeamViewer, and Session Cam tools; 4) a post-test questionnaire/Co-PPGIS polling component for collecting feedback concerning the usability of the prototype; and 5) statistical analysis (interpretation and/or conclusion of statistical results). The procedure is described in detail in Chapter 5.

The case study used a valuable mock-up scenario and helped to explain how prototype applications were understood and used by university students enrolled in different programs, having different levels of expertise and Web participation experiences. The statistical results showed that the prototype system satisfied the objective of the research. The results also showed that the Co-PPGIS Web-based environment is user-friendly and can be easily understandable to non-GIS participants by interpreting correlation results among the usability aspects (i.e., competence, understanding, ease of use, user's contentment and/or satisfaction).

\subsection{Discussion of Thesis Outcomes}

The work presented in this thesis represents a new approach to support public participation and collaborative decision-making. A Co-PPGIS concept supports both synchronous and asynchronous map-sharing participation mechanisms with the integration of GIS and groupware technologies, which are useful for spatial discussions about municipal planning and development-related concerns. The Co-PPGIS has an advantage over the traditional PPGIS and existing participation practices as it allows the collaboration of various stakeholders and the integration of groupware technologies, municipal project information, and data resources. The public from distant locations can work together and co-share not only local data sources, but also knowledge from other users, which has the potential of improving the decision-making process and time and cost efficiency. Similar to other PPGIS, Co-PPGIS does not make the decision itself, but can help improve participation and collaborative decision-making processes. In brief, the research study indicates that the Co-PPGIS concept complements the existing participation practices; and therefore, increases the public participation for bringing about efficiency in planning and decision-related tasks.

As a part of this research, this thesis incorporates requirement engineering standards and UML to the domain of Web online Co-PPGIS, which demonstrates a primary effort in the field of PPGIS design modeling using OOSE. The idea is to provide a contextualized approach at the requirement engineering stage that can contribute to its

effectiveness and widespread acceptance and adoption. A comprehensive elicitation and analysis model is presented, which can be used to identify and elaborate requirements, bring forth the implicit and hidden knowledge of the Co- 
PPGIS concept, discover potential sources of requirements, address needs of all stakeholders and mitigate risks and conflicts from the RE point of view. In addition, this approach presents an extension of UML, called the UWE (UML Web based Engineering) framework for effective analysis of the web navigation and presentation aspects of the Co-PPGIS application. This research provides a clear sequence of activities that can be performed by business analysts/requirement engineers, starting from the vision statement to the correct articulation and analysis of the entire problem. The Co-PPGIS perspective is highlighted throughout this process, from stakeholder identification to context model. The OOSE-based UML approach, described in this research study, should be valuable and suitable as a framework for any other similar efforts.

The Co-PPGIS concept is developed and implemented using OSS-based technologies such as Adobe Flex, Java, PostGreSql/PostGIS, Red5, Blazeds and GeoExt; therefore, it minimizes the potential cost (i.e., a huge early investment and high licensing cost) required to implement the system. Moreover, Co-PPGIS provides cost-effective solutions for smaller municipalities with limited or inadequate financial resources. There is a debate among some groups of people about the negative aspects (e.g., stability, scalability, maintenance and reliability, etc.) of OSS technologies. These aspects were considered during the selection of enabling technologies that were used for the development of the research prototype. In brief, it has been anticipated that with the enhanced functionality, lower cost and more effective communication channels, the research would help to increase the degree to which citizens, municipal bodies, and decision-making authorities can work together using a single platform at the same time.

As far as this prototype is concerned, there are also many limitations in terms of performance and functionality measures because of the limited work force, time, and Internet infrastructure. For example, in CSPE, when many participants are working at the same time, significant Internet speed/ bandwidth is required, which could result in reduced performance due to actual network traffic conditions and unexpected network interruptions. In order to overcome the problem, better data transmission techniques need to be developed and implemented to handle message and data communication between real-time collaborating clients.

This research study was focussed on developing, integrating, and implementing the Co-PPGIS environment along with map-sharing support, in order to facilitate online collaborative discussions within the spatial planning and management context. Component or modular-based design principles were followed in the CSPE and CAPE concepts implementation for this research. The Co-PPGIS integrates social collaboration tools, Web-mapping functionalities, and CSCW/groupware tools and techniques. The research results showed difficulties in finding appropriate software modules that employ groupware and CSCW principles. During system development, special consideration was taken in the selection of enabling technologies as they could increase the throughput (quick 
response) and design/maintenance cost of the system. However, the integration of these tools and technologies was not an easy task, which required proficient programming skills and substantial effort in order to put them together.

Finally, the research also developed the usability evaluation criteria and measured the usability from user's perspective to evaluate quality and performance-based measures of the Co-PPGIS prototype interfaces. This was important before Co-PPGIS prototype deployment in the real-world scenario. Co-PPGIS emphasizes that experts and non-technical users may explore the geographical context of a municipality using GIS. Often, these characteristics necessitate that the systems are accessible, interactive, efficient and user-friendly. The principles of HCI provide motivation regarding the usability of PPGIS applications without compromising the endusers/stakeholders requirements (user's perspective). The current PPGIS application's functions are not usable, to some extent, for non-GIS experts (Haklay and Tobon, 2003). Therefore, the Co-PPGIS prototype components have been designed using HCI principles. Future usability evaluation within real-world scenarios (e.g., testing the system by deploying it in municipal planning and management-related scenarios to identify the need of parking lots in downtown or to find an affordable housing units for low-income groups) will further verify whether or not the CoPPGIS design is usable. Since usability evaluation was not performed in a real municipal project environment, it was difficult to assess the efficiency and satisfaction of decision-making for geographically-spread stakeholders and to measure the time and cost efficiencies achieved through Co-PPGIS.

The research brought together OSS, the Internet, WebGIS, OSGIS, ICT, PPGIS, and groupware, and enhanced current PPGIS frameworks. The outcomes from this research indicate that OSS tools with integration of groupware technologies can be used effectively to facilitate Co-PPGIS environment to support public participation and applied them into real-world municipal planning practices.

\subsection{Contribution and Significance}

This research has addressed the technical and social issues in the design and development of a Co-PPGIS, which little attention has been paid in the field over the last few years. The research outcomes are expected to provide a better understanding of such system in terms of the complexity of the enabling technologies, system design, and the social and human factors involved. In particular, this thesis makes the following contributions:

The thesis is an effort to model a conceptual framework describing the important functional, technological and social aspects for designing and developing collaborative PPGIS. The framework is based on a broad understanding related to existing municipal planning practices, existing PPGIS frameworks, and the special requirements of technologies. Theoretically, the Co-PPGIS framework was developed to identify and define spatial and non-spatial 
data structure, objects and sources needed in a virtual meeting environment along with their required characteristics, such as: composing, connecting and influencing elements. These elements can be divided into: 1) composing elements that include data, software, and mechanism, 2) connecting elements that describe the ways of communication, and 3) influencing elements that address cultural, social, technological or technical aspects.

The current PPGIS does not allow synchronous map-sharing to explore the spatial context related to municipal planning, development, and management projects among participants and decision-making authorities. The present research study is the first effort to integrate both synchronous and asynchronous GIS-based collaboration into PPGIS to facilitate public participation and collaborative decision making during municipal planning related workflows. Such a mechanism will also be helpful in the design and development of emergency response spatial decision support system to support collaborative decision making during disaster risk reduction and/or management.

The study developed a workable, Co-PPGIS research prototype, which integrates multiple open source solutions into a unified virtual meeting environment. One of the unique features of the Co-PPGIS prototype is the integration of a real-time synchronous map sharing component that can facilitate map-based discussions during public meetings. The developed Co-PPGIS prototype also demonstrates the possibility of incorporating groupware, CSCW techniques, OOS technologies, spatial database and map-sharing capabilities onto the WWW infrastructure to support municipal planning and management requirements.

The outcomes/testing results obtained from the usability evaluation of Co-PPGIS provide preliminary measures of implementing user-friendly and efficient interfaces for the stakeholders (i.e., municipal bodies, public, and researchers) that are involved in municipality management related activities. The Co-PPGIS prototype walkthrough, documentation, and understanding about its processes will lead to further research, development, and testing. In addition, the outcome of this research thesis will help other researchers during the advancement of Web-based PPGIS frameworks. 


\subsection{Recommendation for Further Studies}

Several issues related to the Co-PPGIS concept, model and the research prototype may need further work in order to provide alternative solutions for municipal project management, and to support existing planning practices and PPGIS applications. Some of these issues are discussed as follows:

\section{Co-PPGIS Distributed Data Repository}

All spatial and/or non-spatial data involved in the municipal planning-related projects should be stored and managed in a centrally distributed GIS database. However, there are issues with its implementation such as security/ privacy to access the municipal data. For example, the staff access can be limited to only their portion of the database. There is also a need to reduce bandwidth costs with reduced network traffic, and to increase the performance for better and easy handling and management of multiple versions of datasets. The research delivers a valuable starting point towards this Co-PPGIS distributed data-management concept; therefore, further work must be done in evaluation and enhancement.

\section{Usability Evaluation in Real-world Planning and Management Environments}

The research indicated that testing the prototype is needed in a real municipal planning context. Usability evaluation of the Co-PPGIS infrastructure in real-world scenarios is important, especially in real-time environment. The next step of the research will refine the requirements and prototype to work with various real work scenarios. For example, the evaluation of Co-PPGIS for public health and safety, pre-disaster activities, hazardous management, emergency response, mapping the crimes within the city, crop monitoring are all alternative testing scenarios.

\section{Co-PPGIS Implementation and Interpretation as a Mobile-based PPGIS}

As more and more people move to mobile devices, use of mobile-based technologies to make the Co-PPGIS more accessible (as communication channel among public, planner, decision makers, and higher authorities) in real-time during the municipal planning and management activities, e.g., town planning, epidemic control and/or emergency management, becomes next natural step of this kind of participation system. The expansion to support mobile platform will be more technical, but nevertheless requires a lot effort on working around technological hurdles such as technology incompatibility, interoperability, and user interface redesign.

\section{Co-PPGIS Hybrid Mechanism (Offline and Online Views Synchronization)}

Future research is required to develop a hybrid (offline and online views synchronization) mapping mechanism so that some layers are available for all users and spatial datasets can be created and/or viewed even without Internet 
connection. A hybrid framework will help users to create data using application functions without being online and share their local knowledge and data sources in real-time when they have Internet access.

\section{$\underline{\text { Accessible Co-PPIGS }}$}

There is a need to considerably improve, especially the public participation module, to enhance or to accommodate stakeholders with disabilities as they have equal rights to participate in planning and development-related activities. Addressing such an accessibility issue may require comprehensive research on its own, which has not been dealt with in the current study. Avenues of further research for potential expansion of the present research need to be explored. 


\section{References}

- Abdalla R, Li J (2010) Towards effective application of geospatial technologies for disaster management. Int. Journal Applied Earth Observation Geo-information 12:405-407

- Akporiaye O, Ellul C, Cheng T (2010) A Comparison of Spatial Database Functionality, http://wwwresearch.cege.ucl.ac.uk/Posters/2010PosterFair/052-Akporiaye_Omasan.pdf. Last accessed Dec, 2012

- Alexander I, Robertson S (2004) Understanding Project Sociology by Modeling Stakeholders, IEEE Software, 21, No. 1, January/February 2004, 23-27.

- Anderson G, Moreno-Sanchez R 2003, Building web-based spatial information solutions around open specifications and open source software. Transactions in GIS 7(4): 447-466.

- Allen E (2003) Obstacles to community adoption of PPGIS Tools. Proceedings of the 2nd Annual Public Participated GIS Conference, 20-22 July 2003, Portland, Oregon

- Antunes P, Zurita G, Baloian N (2009) A model for designing geocollaborative artifacts and applications. Groupware: Design, Implementation, and Use, 278-294

- Armstrong MP (1994) Requirement for the development of GIS-based group decision support systems. J Am Soc Inf Sci 45:667-677

- Atterer R, Schmidt A, Wnuk M (2007) A Proxy-based Infrastructure for Web Application Sharing and Remote Collaboration on Web Pages. Proceedings of the 11th International Conference on HumanComputer Interaction, Rio de Janeiro, Brazil, September 2007, 74-87, http://murx.medien.ifi.lmu.de/ albrecht/pdf/interact2007-usaproxy.pdf . Accessed 04 April 2009

- Arnstein S (1969) A ladder of citizen participation. Journal of the American Institute of Planners 35: 216224

- Baecker RM (1993) Reading in groupware and computer supported cooperative work: assisting humanhuman collaboration. Morgan Kaufman, San Francisco

- Baker WH, Addams HL, Davis B (2005) Critical factors for enhancing municipal public hearings. Public Adm Rev 65(4):490-499

- Balram S, Dragicevic S (2004) A Web GIS collaborative framework to structure and manage distributed planning processes. J Geogr Syst 6(2):133-153. doi: 10.1007/s10109-004-0130-7

- Ban YU (2003) Public-Participatory Land use decision-making system using Web GIS. Proceedings of the 2nd Annual Public Participated GIS Conference, 20-22 July 2003, Portland, Oregon

- Balram S, Dragicevic S (2006a) Collaborative geographic information systems. Hershey, PA: IGI

- Balram S, Dragicevic S (2006b) Modeling collaborative GIS processes using soft systems theory, UML and object oriented design. Transactions in GIS 10: 199-218

- Balram S, Dragicevic S (2004) A Web GIS collaborative framework to structure and manage distributed planning processes. Journal of Geographical Systems 6(2):133-153 doi:10.1007/s10109-004-0130-7

- Balram S, Dragicevic S, Meredith T (2003) Achieving effectiveness in stakeholder participation using the GIS-based collaborative spatial Delphi methodology. Journal of Environmental Assessment Policy and Management 5: 365-94

- Banati H, Bedi P, Grover PS (2006) Evaluating Web usability from the user's perspective. Journal of Computer Science 2(4): 314-317

- Bevan N (1995) Measuring usability as quality of use. Journal of Software Quality 4(2):115-130

- Bennett S, McRobb S, Farmer R (2005) Object-oriented systems analysis and design using UML. McGraw Hill Higher Education. http://www.greymatter.com/133000

- Begole J, Struble CA, Shaffer C, Smith RB (1999) System resource sharing for synchronous collaboration. Technical Report TR-99-11, Computer Science, Virginia Tech. http://eprints.cs.vt.edu:8000/archive/00000524/01/BetEtA199b.pdf. Accessed Oct 2011 
- Bill R, Korduan P (2004) Internet-GIS Development for Municipalities and Counties based on Open Source Software. International Society for Photogrammetry and Remote Sensing. XXth ISPRS Congress, 12-23 July 2004 Istanbul, Turkey

- Blaschke T (2004) Participatory GIS for spatial decision support systems critically revisited. In GIScience 2004. Ed. M. Egenhofer, C. Freksa, H. Miller. University of Maryland Conference Center, October 20-23, 2004. pp. 257-261 http://map3.salzburgresearch.at/docs/GI_Science_Blaschke.pdf. Accessed August 05

- Booch G, Rumbaugh J, Jacobson I (2005) The unified modeling language user guide, Upper Saddle River, New Jersey, $475 \mathrm{p}$

- Boroushaki S and Malczewski J (2009) ParcitipatoryGIS.com: A WebGIS-based Collaborative Multicriteria Decision Analysis, Submitted to URISA Journal, http://www.urisa.org/files/Boroushaki\%20\%20ParcitipatoryGIS\%20com\%20(2).pdf (last visited April 29, 2009)

- Boroushaki S, Malczewski J (2010) Measuring consensus for collaborative decision-making: a GIS-based approach. Computer Environment Urban System 34:322-332

- Boulos M, Warren J, Jianya G, Peng Y (2010) Web GIS in practice VIII: HTML5 and the canvas element for interactive online mapping. Int J Health Geography 9:14-26

- Brail RK, Klosterman RE (2001) Planning support systems: integrating geographic information systems, models, and visualization tools. ESRI, Redlands

- Bryant L, Wilcox D (2006) Some lessons from Web 2.0 for participation and democracy. http://www.headshift.com/archives/002787.cfm. Accessed June 2006

- Butler KA (1996) Usability engineering turns 10. Interactions 3(1):58-75

- Butt MA, Li S (2015) UML-based requirement modeling of Web online synchronous collaborative public participatory GIS. Journal of Applied Geomatics. DOI 10.1007/s12518-015-0154-3

- Butt MA, Li S (2014) Usability evaluation of collaborative PPGIS-GeoCWMI for supporting public participation during municipal planning and management services. Journal of Applied Geomatics (2014): $1-23$

- Butt MA, Li S (2012) Open source based online map-sharing to support real-time collaboration, OSGeo Journal 2012 (10): 5-14

- Carver S (2003) The future of participatory approaches using geographic information: Developing a Research Agenda for the 21st Century. Journal of Urban and Regional Information System, Special Issue, 15, Access and Participatory Approaches

- Carver S (2001) Participation and geographical information: a position paper. Workshop on Access to Geographic Information and Participatory Approaches Using Geographic Information, Spoleto, 6-8 December 2001

- Chang Z (2010) Synchronous collaborative 3D GIS with agent support. Ph.D. Thesis, Ryerson University, Toronto, Canada

- Chua YL and Wong S (2002) Data intermediation and beyond: how the Web modifies the dissemination of GIS information, Proceedings of the 2nd Public Participation GIS Conference, New Brunswick, U.S.A, July 2002, http://www.iapad.org/publications/ppgis/swong.pdf (last visited April 29, 2009)

- Chung G, Jeffay K, Abdel-Wahab H (1994) Dynamic participation in computer-based conferencing system. J Comput Commun 17(1):7-16

- Churcher N, Churcher C (1999) Realtime conferencing in GIS. Trans GIS 3(1):23-30

- Churcher N, Churcher C (1996) GroupARC - a collaborative approach to GIS, proceedings of 8th annual colloquium of the spatial information research center, University of Otago, New Zealand, July 9-11, 1996, pp. $156-163$

- CIAC (2008) Putting the people into planning: a primer on public participation in planning. DLCD. Salem, OR: Oregon's Citizen Involvement Advisory Committee 
- Connor DM (1996) Public participation in Canada: development, current status and trends, URL: http://www.connor.bc.ca/ppcanada/ppcanada.html, Victoria, BC (last date accessed: 05 March 2007)

- Craig WJ, Elwood SA (1998) How and why community groups use maps and geographic information. Cartogr Geogr Inf Syst 25(2):95-104

- Craig WJ, Harris TM, Weiner D (eds) (2002) Community participation and geographic information science. Taylor and Francis, London

- Dix A, Finlay J, Abowd G, Beale R (1998) Human-Computer Interaction (Second Edition) Prentice Hall.

- De Meo I, Cantiani MG, Ferretti F, Paletto A (2011). Stakeholders' perception as support for forest landscape planning. International Journal of Ecology, Article ID 685708. - doi: 10.1155/2011/685708

- Dragicevic S (2004) The potential of Web-based GIS. J Geogr Syst 6:79-81

- Dragicevic S, Balram S (2004) A Web GIS collaborative framework to structure and manage distributed planning processes. J Geogr Syst 6:133-153

- Evans A, Kingston R, Carver S, Turton I (1999) Web-based GIS used to enhance public democratic involvement. Geocomp'99 Conference Proceedings, Mary Washington College, Virginia, USA, July 27-28 1999

- Faber BG, Wallace WW, Johnson GE (1998) Active response GIS: for resource management spatial decision support systems. Photogrammetric Engineering and Remote Sensing 64:7-11

- Fiedrich F, Burghardt P (2007) Agent-based systems for disaster management. Commun ACM 50:41-42

- Fortin N (2008) The gender wage gap among young adults in the United States, Journal of Human Resources 43 884-918

- Ghose R (2001) Use of information technology for community empowerment: Transforming geographic information systems into community information systems. Transactions in GIS 5:141-63

- Grassmuck, Volker (2004) Freie Software. Zwischen Privat- und Gemeineigentum, Bundeszentrale für politische Bildung Schriftenreihe Band 458, 2. Auflage, Bonn 2004

- Gu L (2004) Development of an internet-based synchronous GIS for collaborative spatial decision-making. Master Thesis, Ryerson University, Toronto, Canada.

- Hackney's GIS Team (2004) Map Hackney, U.K. http://www.map.hackney.gov.uk/. Accessed August 2004

- Hall GB, Leahy MG (2008) Design and implementation of a map-centred synchronous collaboration tool using open source components: the MapChat project. In G. Brent Hall and Michael G. Leahy (eds.) Open Source approaches to spatial data handling. Springer, Berlin, pp. 221-245

- Hall GB, Leahy MG (2006) Internet-based spatial decision support using Open Source tools. In: Balram S, Dragicevic S (eds) Collaborative geographic information systems, Idea Group Publishing, Hershey, pp 237-262

- Haklay M, Tobón C (2003) Usability evaluation and PPGIS: towards a user-centered design approach. Int J Geogr Inf Sci 17(6):577-592

- Haklay M, Zafiri A (2008) Usability engineering for GIS: learning from a screenshot. Cartogr J 45(2):8797

- Healey P (1997) Collaborative planning: shaping places in fragmented societies. Macmillan, London

- Hopkins LD, Twidale M and Pallathucheril VG (2004) Interface devices and public participation, Proceedings of the 3rd Annual PPGIS Conference of Urban and Regional Information Systems Association, Madison, United States, 71-83

- Huxhold W (1991) An introduction to urban geographic information systems. Oxford University Press, New York

- ISO (International Organization for Standardization) (1998) Ergonomic Requirements for Office Work with Visual Display Terminals -- Part 11: Guidance on Usability, ISO 
- Jankowski P, Nyerges T (2001a) Geographic information systems for group decision making: Towards a Participatory Geographic Information Science. New York, Taylor and Francis

- Jankowski P, Nyerges T (2001b) GIS-Supported collaborative decision making: Results of an experiment. Annals of the Association of American Geographers 91: 48-70

- Jankowski P, Nyerges T (2003) Toward a framework for research on geographic information-supported participatory decision-making. URISA Journal 15 (1):9-17

- Jankowski P (2009) Towards participatory geographic information systems for community-based environmental decision-making. Journal of Environmental Management 90(6):1966-1971

- Jankowski P, Nyerges T, Smith A, Moore TJ, Horvath E (1997) Spatial group choice: a SDSS tool for collaborative spatial decision making. Int. Journal of Geography Information System 11(6):577-602

- Jarke M, Pohl K (1994) Requirements Engineering in 2001: (virtually) managing a changing reality. Software Engineering Journal (November 1994)

- Jiansheng G (2007) Mashing up web-based participatory GIS: Potential for future e-government services. Master Thesis, Ryerson University, Toronto, Canada

- Johansen R (1988) Groupware: Computer Support for Business Teams. The Free Press.

- Jones RM, Copas CV, Edmonds EA (1997) GIS support for distributed group-work in regional planning. Int J Geogr Inf Sci 11(1):53-71

- Jordan PW (1998) An introduction to usability. CRC, Boca Raton

- Kao Y and Lin G (1998) Development of a collaborative CAD/CAM system, Robotics and Computer integrated Manufacturing, 14 (1998), pp. 55 - 6

- Keßler C (2004) Design and implementation of argumentation maps. Master's thesis, Institute for Geoinformatics, Westfälische Wilhelms-Universität Münster, Germany. http://www.carstenkessler.de/argumap. Accessed 30 June 2005

- Keßler C, Rinner C, Raubal M (2005) An argumentation map prototype to support decision making in spatial planning. Proceedings of the 8th Association of Geographic Information Laboratories for Europe (AGILE) Conference on GIScience, Estoril, Portugal: 26-8

- Kingston R (2002) Web-based PPGIS in the United Kingdom. In: Craig WJ, Trevor TM, Weiner D (eds) Community participation and geographic information systems. Taylor \& Francis, London, pp 101-112

- Kingston R, Carver S, Evans A, Turton I (2000) Web-based public participation geographical information systems: an aid to local environmental decision-making. Computers, Environment and Urban Systems 24 (2): $109-125$

- Kingston R (2007) Public participation in local policy decision making: The role of Web-based mapping. The Cartographic Journal 44(2): 138-44

- Klosterman RE (2001) Planning support systems. ESRI, Redlands, pp 1-23

- Lakhani K, Von HE (2003) How open source software works: "Free" user-to-user assistance. Research Policy (forthcoming)

- Laurini R (2004) Computer systems for public participation. Available online from http://www.gisig.it/vpc_sommet/CD_Sommet/ws3/articololaurini.pdf

- Lemos M (2006) PHP Clases blog: Is PHP ready for Web 2.0. http://www.phpclasses.org/blog/post/53-IsPHP-ready-for-Web-20.html. Accessed 31 May 2014

- Li S, Guo X, Ma X, Chang Z (2007) Towards GIS-enabled virtual public meeting space for public participation, Photogrammetric Engineering \& Remote Sensing 73(6)641-650

- Li S, Ma X (2006) An open source GIS solution for supporting public participation in municipal developments, Proceedings of the ISPRS Commission IV Symposium on Geospatial Databases for Sustainable Development, 27-30 September 2006, Goa, India, pp. 1056-1060 
- Li S, Chang Z, Yi R (2004) GIS-based internet notice board to facilitate public participation in municipal developments, Proceedings of the 20th ISPRS Annual Congress, 12-23 July 2004, Istanbul, Turkey, pp. 269-274

- Li S (2002) Design and development of an internet collaboration system to support distributed GIS data production management, Ph.D. Dissertation, University of New Brunswick, Fredericton, N.B., Canada, 275 $\mathrm{p}$

- Longley P, Goodchild M, Maguire DJ, Rhind DW (2010) Geographic information systems and science. John Wiley \& Sons.

- Lowndes V, Pratchett L, Stoker G (2001) Trends in public participation: part 1-citizen's perspectives. Public Adm 79(2):445-455

- Lynn FM, Busenberg GJ (1995) Citizen advisory committees and environmental policy: what we know, what's left to discover. Risk Anal 15(2):147-162

- Ma X (2006) A prototype of Web-based PPGIS for municipal planning using open source software. Master Thesis, Ryerson University, Toronto, Canada

- MacEachren AM, Brewer I, Steiner E (2001) Geo-visualization to mediate collaborative work: Tools to support different-place knowledge construction and decision-making. http://hero.geog.psu.edu/products/ICC16009amm.pdf. Accessed 5 June 2010

- MacEachren AM (2000) Cartography and GIS: facilitating collaboration. Prog Hum Geogr 24(1):445-456

- Macintosh A (2004) Characterizing e-participation in policy-making. Proceedings of the 37th Annual Hawaii International Conference on System Sciences (HICSS-37), Big Island, Hawaii January 5 - 82004

- Maguire M, Kirakowski J, Vereker N (1998) Respect: user-centred requirements handbook. HUSAT Research Centre, Loughborough

- Matera M, Rizzo F, Carughi GT (2005) Web usability: principles and evaluation methods. In: Web engineering. Springer, Heidelberg, pp 109-142

- McClure DL (2000) Statement of David L. McClure, U.S. general accounting office, before the subcommittee on Government management, information and technology, committee on Government reform, House of Representatives. Available: http://www.gao.gov

- Meng Y, Malczewski J (2009) Usability evaluation for A Web-based public participatory GIS: A case study in Canmore, Alberta' Cybergeo: European Journal of Geography Article 483, http://www.cybergeo.eu/index22849.html

- Meredith TC (2000) Community participation in environmental information management: exploring tools for developing an impact assessment preparedness program, a report from Canadian environmental assessment agency, URL: http://www.ceaa.gc.ca/015/0002/0016/print-version_e.htm. Accessed 25 April 2004

- Newton P, Zwart P, Cavil M (1995) Networking Spatial Information Systems, Revised Edition, John Wiley $\&$ Sons Ltd., England, UK

- Nielsen J (1993) Usability Engineering. Academic Press, Boston, MA.

- Nielsen J (2000) Designing web usability. Indiana: New Riders Publishing.

- Obermeyer NJ (1998) The evolution of public participation GIS. Cartography and Geographic Information Systems 25 (2):65-66

- Orlando GIS (2004) Orange county INFOMAP interactive mapping. http://ocgis1.ocfl.net/imf/imf.jsp?site=orangecFL. Accessed July 2004

- OSI (2007) The Open Source Initiative. http://opensource.org/2007. Last accessed Dec 2012

- Pang A, Fernandez D (1995) REINAS instrumentation and visualization. Proceedings, OCEANS'95. MTS/IEEE. Challenges of our changing global environment, San Diego, Oct. 9-15, pp. 1892-1899

- Peng ZR (2001) Internet GIS for public participation. Environment and Planning B: Planning and Design 2001,28: 889-905 
- Pohl K (1994) Requirements Engineering: An Overview. Encyclopaedia of Computer Science and Technology, A. Kent, J. Williams, and C.M. Hall, eds., M. Dekker, New York, vol. 36, 345-386

- Preece J (1993) A guide to usability: human factors in computing. Addison-Wesley, Boston

- Preece J, Yvonne R, Helen S (2002) Interaction design: beyond human-computer interaction. Wiley, New York

- Rinner C, Kebler C, Andrulis S (2008) The use of Web 2.0 concepts to support deliberation in spatial decision-making. Computers, Environment and Urban System 32(5): 386-395

- Rinner C (2006a) Mapping in collaborative spatial decision making. Collaborative geographic information systems. Idea Group Publishing, Hershey, pp 85-102

- Rinner C (2006b) Argumentation mapping in collaborative spatial decision making. In: Balram S, Dragicevic S (eds) Collaborative geographic information systems. Idea Group Publishing, Hershey, pp 85102

- Rinner C (2001) Argumentation Maps - GIS-based discussion support for online planning, Environment and Planning B: Planning and Design 28(6) 847-863

- Rinner C (1999) Argumentation maps - GIS-based discussion support for online planning, Ph.D. Dissertation, University of Bonn, Germany

- Roche S, Mericskay B, Batita W, Bach M, Rondeau M (2012) WikiGIS Basic Concepts: Web 2.0 for Geospatial Collaboration. Future Internet 4: (265-284) doi:10.3390/fi4010265

- Rojas E and Songer A (1999) Web-centric systems: a new paradigm for collaborative engineering, Journal of Management in Engineering, 15(1), Jan/Feb

- Sadagopan GD (2000) Web-based geographic information system: public participation in virtual decision making environment. Master Thesis of Virginia Polytechnic Institute and State University

- Sano J, Wanalertlak N, Maki A, Minoura T (2003) Benefits of web-based GIS/database applications. Proceedings of 2nd Annual Public Participation GIS Conference, Portland, Oregon, July 2003

- Scientific A (1990) Special issue on communications. Comput Netw 265(3)

- Schwedes S, Werner W (2010) Manual for participatory land use planning facilitators. http://www.iapad.org/publications/ppgis/Manual_PLUP\%20Namibia_final_01_09.pdf. Accessed 25 April 2012

- Schlossberg MA (1998) Asset mapping and community development planning with GIS: a look at the heart of West Michigan United Way's innovative approach. Association for Research on Nonprofit Organizations and Voluntary Action, Annual Conference, Nov 5-7. Retrieved August 20, 2003, from http://www.uoregon.edu/ schlossb/PPPM/gis/gis_uw.pdf.

- Shneiderman B (1998) Designing the user interface. Addison-Wesley Longman, Boston

- Shipley R, Utz S (2012) Making It Count: A Review of the Value and Techniques for Public Consultation. Journal of Planning Literature 27 (1) (January 17): 22-42. doi:10.1177/0885412211413133. http://jpl.sagepub.com/cgi/doi/10.1177/0885412211413133.

- Simão A, Densham PJ, Haklay M (2009) Web-based GIS for collaborative planning and public participation: an application to the strategic planning of wind farm sites. Journal of Environmental Management 90(6): 2027-2040

- Sieber R (2006) Public participation geographic information systems: A literature review and framework. Annals of the Association of American Geographers 96 (3):491-507

- Sidlar C, Rinner C (2007) Analyzing the Usability of an Argumentation Map as a Participatory Spatial Decision Support Tool URISA Journal, Vol.19, No.1, 47-55.

- Smotritsky Y (2004) Running GIS on Open Source, Connect: Information Technology at NYU, Fall/Winter 2004, 17-20. 
- Sullivan JM, Hall R, Hilgers M, Luna R, Buechler MR, Lawrence WT (2005) Iterative Usability Evaluation Methods Applied to Learning Technology Development. In World Conference on Educational Multimedia, Hypermedia and Telecommunications (EDMEDIA), 2005

- Sullivan EJ (1998) Remarks to university of Oregon symposium marking the 25th anniversary of SB 100.

- Sweeney M, Maguire M, Shackel B (1993) Evaluating user-computer interaction: a framework. Int J ManMach Stud 38:689-711

- Tang T (2006) Design and implementation of a GIS-enabled online discussion forum for participatory planning. M.Sc.E. thesis, Department of Geodesy and Geomatics Engineering Technical Report No. 244, University of New Brunswick, Fredericton, New Brunswick, Canada, 151 pp

- Tang MY, Coleman DJ (2005) Design of a GIS-enabled online discussion forum for participatory community planning, Proceedings of the 98th Annual Conference of the Canadian Institute of Geomatics, Ottawa, Canada, June 2005

- Tang T, Zhao J, Coleman DJ (2005) Design of a GIS-enabled online discussion forum for participatory planning. In Proceedings of the 4th Annual Public Participation GIS Conference, Cleveland, Ohio, USA, July 2005

- Talen E (1999) Constructing neighbourhoods from the bottom up: The case for resident-generated GIS. Environment and Planning B: Planning and Design 26(4): 533-54.

- Thomas C, Bevan N (1996) Usability context analysis: a practical guide. Serco Usability Services, Teddington

- Tritter JQ, McCallum A (2006) The snakes and ladders of user involvement: moving beyond Arnstein. Health Policy, 76:156-168.

- Tsou MH, Smith J (2011) Free and Open Source Software for GIS education, A White Paper.http://www.iapad.org/publications/ppgis/tsou_free-GIS-foreducators-whitepaper.pdf. Last accessed Nov 2012

- Turner A 2005 GIS Options: RELU Upland Moorland Scoping Study Project. University of Leeds, UK. [Online]. Available from: http://www.geog.leeds.ac.uk/people/a.turner/publications/GIS\%20Options\%20Working\%20Paper.pdf. Accessed: March 25, 2012.

- Vari A (1996) Citizens' advisory committee as a model for public participation: a multiple-criteria evaluation. In: Renn O, Webler T, Wiedemann P (eds) Fairness and competence in citizen participation: evaluating models for environmental discourse. Kluwer Academic Publishers, Dordrecht, pp 103-116

- Ventura SJ, Niemann BJ, Sutphin TL, Chenoweth RE (2002) GIS enhanced land use planning. In: Craig WJ, Harris TM, Weiner D (eds) Community participation and geographic information systems. Taylor \& Francis, London, pp 113-124

- Voss A, Denisovich I, Gatalsky P, Gavouchidis K, Klotz A, Roeder S, Voss H (2004) Evolution of a participatory GIS. Computers, Environment and Urban Systems 28 (6):635-651

- Wang D, Xu K (2010) Red5 Flash server analysis and video call service implementation, Web Society (SWS), 2010 I.E. 2nd Symposium on 16-17 Aug. 2010

- Warren-Kretzschmar B, Tiedtke S (2005) What Role Does Visualization Play in Communication with Citizens? A Field Study from the Interactive Landscape Plan. In Trends in Real-Time Landscape Visualization and Participation, edited by E. Buhmann, 156-167. Heidelberg: Herbert Wichmann Verlag

- Wangmutitakul P, Li L, Minoura T (2003) User participatory Web-based GIS/database application. Proceedings of Geotech Event Conference, Vancouver, British Columbia, March 16-19 2003

- Wangmutitakul P et al. (2004) WebGD: framework for Web-based GIS/database applications. J Object Technol 3(4):209-225

- Wiedemann PM, Femers S (1993) Public participation in waste management decision making: analysis and management of conflicts. J Hazard Mater 33(3):355-368 
- Wong S, Chua YL (2001) Data intermediation and beyond: issues for web-based PPGIS. Cartographica 38(3):4

- Wuttiwat T, Minoura T, Steiner J (2003) Using digital orthographic aerial images as user interfaces. Proc. of ASPRS Annual Conference, Anchorage, Alaska, May 2003

- Zhao J, Coleman DJ (2006) GeoDF: Towards a SDI-based PPGIS application for e-governance. Proceedings of the GSDI 9 Conference, Santiago, Chile. http://www.gsdidocs.org/gsdiconf/GSDI9/papers/TS9.3paper.pdf. Accessed 7 Dec 2010

- Zhao J (2007) An empirical usability evaluation of a Web-based public participation geographic information system and discussion forum. M.Sc.E. thesis, Department of Geodesy and Geomatics Engineering Technical Report No. 250, University of New Brunswick, Fredericton, New Brunswick, Canada, 155 pp session Tool 


\section{APPENDIX 1 Participant's Basic Information Pre-Test Questionnaire}

Note: This questionnaire is designed for testing and evaluating Co-PPGIS Map-sharing System application usability which is solely for research purposes. The information thus gathered from the users will be kept confidential. Thank you for your cooperation.

1. What educational program you're enrolled in?

- Master degree

- M.Phil. degree

- Doctoral degree

2. What is your Major subject?

3. Gender:

- Male

- Female

4. How would you rank your knowledge level of computer/Internet?

- None

- Basics

- Moderate

- Good

- Very good

5. How would you rank your knowledge level of GIS?

- None

- Basics

- Moderate

- Good

- Very good

6. Have you heard or used any of the following web-mapping systems?

- Google Maps

- Google Earth

- Yahoo Maps

- None of the above

- Other, please specify 


\section{APPENDIX 2 Feedback Questionnaire (Post-Test Questionnaire)}

Note: Kindly mark your responses on how did you find this new web-based application designed for the purpose of Web-based Co-PPGIS Map-sharing System.

1. Do you think you have gained web-based GIS knowledge after working on Co-PPGIS?
Agree
(2)
Disagree (1)
Neutral (0)

2. Is the Co-PPGIS web-application a common platform for public participation?
Agree
(2)
Disagree (1)
Neutral (0)

3. Did you find the instructions easy enough to explore the Co-PPGIS web-based application?
Agree
(2)
Disagree (1)
Neutral (0)

4. Was it easy for you to complete the given assignment on time?
Agree
(2)
Disagree (1)
Neutral (0)

5. Did you find the mapping sharing tool relevant to this forum?
Agree
(2)
Disagree (1)
Neutral (0)

6. Co-PPGIS allowed you to find locations on map, comment, draw and chat simultaneously with others?
Agree
(2)
Disagree (1)
Neutral (0)

7. Did you find the online discussion forum of this application effective?
Agree
(2)
Disagree (1)
Neutral (0)

8. Did you feel distracted (lost interest at some stage) while performing the tasks?
Agree
(2)
Disagree (1)
Neutral (0)

9. Did you find the web-based application innovative and simple to operate?
Agree
(2)
Disagree (1)
Neutral (0)

10. Did you find this web-based application helpful in mutual cooperation?
Agree
(2)
Disagree (1)
Neutral (0) 
APPENDIX 3 Tabular Display of Results Depicting User Profile and Performance

Table I: Results Showing Participant Profile Obtained Through Pre-Questionnaire

\begin{tabular}{|l|l|}
\hline \multicolumn{2}{|c|}{ Gender Ratio (Students) } \\
\hline Males & Females \\
\hline 9 & 16 \\
\hline
\end{tabular}

\begin{tabular}{|l|l|}
\hline Educational Degree & $\begin{array}{l}\text { No. of } \\
\text { Participants }\end{array}$ \\
\hline Graduate & 12 \\
\hline Post-Graduate & 12 \\
\hline Doctorate & 1 \\
\hline
\end{tabular}

\begin{tabular}{|l|l|}
\hline GIS knowledge & $\begin{array}{l}\text { No. of } \\
\text { Participants }\end{array}$ \\
\hline Nil & 6 \\
\hline Beginner & 9 \\
\hline Moderate & 4 \\
\hline Good & 1 \\
\hline V. Good & 5 \\
\hline
\end{tabular}

\begin{tabular}{|l|l|}
\hline IT Knowledge & $\begin{array}{l}\text { No. of } \\
\text { Participants }\end{array}$ \\
\hline Nil & 0 \\
\hline Beginner & 1 \\
\hline Moderate & 6 \\
\hline Good & 15 \\
\hline V. Good & 3 \\
\hline
\end{tabular}

Table II: Group-wise Results of User Performance

\begin{tabular}{|l|l|l|l|l|}
\hline Groups & $\begin{array}{l}\text { Total No. } \\
\text { of Clicks }\end{array}$ & $\begin{array}{l}\text { Avg. Time } \\
\text { (min) to } \\
\text { Perform a } \\
\text { Single Task }\end{array}$ & $\begin{array}{l}\text { Avg. Clicks } \\
\text { to Perform a } \\
\text { Single Task }\end{array}$ & $\begin{array}{l}\text { No. of Tasks } \\
\text { Performed }\end{array}$ \\
\hline Urban Planning & 57 & 12 & 16 & 4 \\
\hline Environmental Engineering & 54 & 12 & 16 & 4 \\
\hline GIS & 38 & 7 & 8 & 5 \\
\hline Management Studies & 58 & 13 & 16 & 4 \\
\hline Eng. Literature & 60 & 13 & 21 & 3 \\
\hline
\end{tabular}




\section{APPENDIX 4 Spearman Rank Correlation}

Table I: Correlations Between User Profile and User Performance

\begin{tabular}{|c|c|c|c|c|c|c|c|c|c|c|}
\hline & & & Education & $\begin{array}{l}\text { GIS } \\
\text { Knowledge }\end{array}$ & $\begin{array}{l}\text { IT } \\
\text { Knowledge }\end{array}$ & $\begin{array}{l}\text { Total Time } \\
\text { Consumed (min) }\end{array}$ & $\begin{array}{l}\text { Total No. of } \\
\text { Clicks }\end{array}$ & $\begin{array}{l}\text { Avg. Time (min) to } \\
\text { Perform a Single } \\
\text { Task }\end{array}$ & $\begin{array}{l}\text { No. of Tasks } \\
\text { Performed }\end{array}$ & $\begin{array}{l}\text { User } \\
\text { Contentment }\end{array}$ \\
\hline \multirow{18}{*}{$\begin{array}{l}\text { Spearman's } \\
\text { rho }\end{array}$} & \multirow[t]{3}{*}{ Education } & Correlation Coefficient & 1.000 & -.087 & -.180 & -.207 & .184 & -.054 & -.143 & .129 \\
\hline & & Sig. (2-tailed) & & .678 & .389 & .320 & .378 & .799 & .495 & .540 \\
\hline & & $\mathrm{N}$ & 25 & 25 & 25 & 25 & 25 & 25 & 25 & 25 \\
\hline & \multirow[t]{3}{*}{ GIS Knowledge } & Correlation Coefficient & -.087 & 1.000 & $.593^{* *}$ & $-.738^{* *}$ & $-.634^{* *}$ & $-.695^{* *}$ & $.714^{* *}$ & $-.558^{* *}$ \\
\hline & & Sig. (2-tailed) & .678 & . & .002 & .000 & .001 & .000 & .000 & .004 \\
\hline & & $\mathrm{N}$ & 25 & 25 & 25 & 25 & 25 & 25 & 25 & 25 \\
\hline & \multirow[t]{3}{*}{ IT Knowledge } & Correlation Coefficient & -.180 & $.593^{* *}$ & 1.000 & -.389 & $-.499^{*}$ & $-.541^{* * *}$ & $.626^{* *}$ & -.257 \\
\hline & & Sig. (2-tailed) & .389 & .002 & 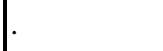 & .055 & .011 & .005 & .001 & .215 \\
\hline & & $\mathrm{N}$ & 25 & 25 & 25 & 25 & 25 & 25 & 25 & 25 \\
\hline & \multirow{3}{*}{$\begin{array}{l}\text { Total Time } \\
\text { Consumed (min) }\end{array}$} & Correlation Coefficient & -.207 & $-.738^{* *}$ & -.389 & 1.000 & $.421^{*}$ & $.670^{* *}$ & $-.490^{*}$ & $.463^{*}$ \\
\hline & & Sig. (2-tailed) & .320 & .000 & .055 & . & .036 & .000 & .013 & .020 \\
\hline & & $\mathrm{N}$ & 25 & 25 & 25 & 25 & 25 & 25 & 25 & 25 \\
\hline & \multirow[t]{3}{*}{ Total No. of Clicks } & Correlation Coefficient & .184 & $-.634^{* *}$ & $-.499^{*}$ & $.421^{*}$ & 1.000 & $.580^{* *}$ & $-.599^{* *}$ & .240 \\
\hline & & Sig. (2-tailed) & .378 & .001 & .011 & .036 & & .002 & .002 & .248 \\
\hline & & $\mathrm{N}$ & 25 & 25 & 25 & 25 & 25 & 25 & 25 & 25 \\
\hline & \multirow{3}{*}{$\begin{array}{l}\text { Avg. Time (min) to } \\
\text { Perform a Single } \\
\text { Task }\end{array}$} & Correlation Coefficient & -.054 & $-.695^{* *}$ & $-.541^{* * *}$ & $.670^{* *}$ & $.580^{* *}$ & 1.000 & $-.795^{* *}$ & $.436^{*}$ \\
\hline & & Sig. (2-tailed) & .799 & .000 & .005 & .000 & .002 & & .000 & .029 \\
\hline & & $\mathrm{N}$ & 25 & 25 & 25 & 25 & 25 & 25 & 25 & 25 \\
\hline
\end{tabular}




\begin{tabular}{|c|c|c|c|c|c|c|c|c|c|}
\hline $\begin{array}{l}\text { No. of Tasks } \\
\text { Performed }\end{array}$ & $\begin{array}{l}\text { Correlation Coefficient } \\
\text { Sig. (2-tailed) } \\
\mathrm{N}\end{array}$ & $\begin{array}{l}-.143 \\
.495 \\
25\end{array}$ & $\begin{array}{l}.714^{* *} \\
.000 \\
25\end{array}$ & $\begin{array}{l}.626^{* *} \\
.001 \\
25 \\
\end{array}$ & $\begin{array}{l}-.490^{*} \\
.013 \\
25\end{array}$ & $\begin{array}{l}-.599^{* *} \\
.002 \\
25\end{array}$ & $\begin{array}{l}-.795^{* *} \\
.000 \\
25\end{array}$ & $\begin{array}{l}1.000 \\
25\end{array}$ & $\begin{array}{l}-.306 \\
.137 \\
25\end{array}$ \\
\hline User Contentment & $\begin{array}{l}\text { Correlation Coefficient } \\
\text { Sig. (2-tailed) } \\
\mathrm{N}\end{array}$ & $\begin{array}{l}.129 \\
.540 \\
25\end{array}$ & $\begin{array}{l}-.558^{* *} \\
.004 \\
25\end{array}$ & $\begin{array}{l}-.257 \\
.215 \\
25\end{array}$ & $\begin{array}{l}.463^{*} \\
.020 \\
25\end{array}$ & $\begin{array}{l}.240 \\
.248 \\
25\end{array}$ & $\begin{array}{l}.436^{*} \\
.029 \\
25\end{array}$ & $\begin{array}{l}-.306 \\
.137 \\
25\end{array}$ & $\begin{array}{l}1.000 \\
25\end{array}$ \\
\hline
\end{tabular}

*. Correlation is significant at the 0.05 level (2-tailed)

**. Correlation is significant at the 0.01 level (2-tailed).

Table II: Correlation Between Variables of User Satisfaction and User Performance

\begin{tabular}{|c|c|c|c|c|c|c|c|c|c|c|c|c|c|}
\hline . & 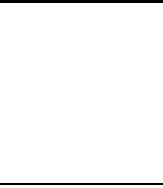 & Innovative & Gender & $\begin{array}{l}\text { Group } \\
\text { of User }\end{array}$ & Education & $\begin{array}{l}\text { Total } \\
\text { Time } \\
\text { Consumed } \\
\text { (min) }\end{array}$ & $\begin{array}{l}\text { Total } \\
\text { No. of } \\
\text { Clicks }\end{array}$ & $\begin{array}{l}\text { Avg. } \\
\text { Clicks to } \\
\text { Perform } \\
\text { a Single } \\
\text { Task }\end{array}$ & $\begin{array}{l}\text { No. of } \\
\text { Tasks } \\
\text { Performed }\end{array}$ & $\begin{array}{l}\text { Learn- } \\
\text { ability }\end{array}$ & $\begin{array}{l}\text { Content } \\
\text { Relevance }\end{array}$ & $\begin{array}{l}\text { Communica- } \\
\text { tive }\end{array}$ & $\begin{array}{l}\text { Partici- } \\
\text { patory }\end{array}$ \\
\hline \multirow{3}{*}{ Innovative } & $\begin{array}{l}\text { Correlation } \\
\text { Coefficient }\end{array}$ & 1 & 0.178 & 0.241 & 0.335 & 0.263 & 0.201 & 0.204 & -0.154 & & -0.33 & 0.025 & -0.269 \\
\hline & $\begin{array}{l}\text { Sig. (2- } \\
\text { tailed) }\end{array}$ & . & 0.396 & 0.246 & 0.101 & 0.204 & 0.334 & 0.327 & 0.463 & & 0.107 & 0.905 & 0.193 \\
\hline & $\mathrm{N}$ & 25 & 25 & 25 & 25 & 25 & 25 & 25 & 25 & 25 & 25 & 25 & 25 \\
\hline \multirow{3}{*}{ Gender } & $\begin{array}{l}\text { Correlation } \\
\text { Coefficient } \\
\end{array}$ & 0.178 & 1 & $-.589^{* *}$ & 0.366 & -0.224 & -0.185 & -0.098 & 0.128 & & -0.368 & -0.326 & 0.393 \\
\hline & $\begin{array}{l}\text { Sig. (2- } \\
\text { tailed) }\end{array}$ & 0.396 & & 0.002 & 0.072 & 0.283 & 0.376 & 0.64 & 0.542 & & 0.07 & 0.112 & 0.052 \\
\hline & $\mathrm{N}$ & 25 & 25 & 25 & 25 & 25 & 25 & 25 & 25 & 25 & 25 & 25 & 25 \\
\hline Education & $\begin{array}{l}\text { Correlation } \\
\text { Coefficient }\end{array}$ & 0.335 & 0.366 & -0.049 & 1 & -0.207 & 0.184 & 0.256 & -0.143 & & 0.006 & -0.187 & -0.127 \\
\hline
\end{tabular}




\begin{tabular}{|c|c|c|c|c|c|c|c|c|c|c|c|c|c|}
\hline & $\begin{array}{l}\text { Sig. (2- } \\
\text { tailed) }\end{array}$ & 0.101 & 0.072 & 0.817 & . & 0.32 & 0.378 & 0.216 & 0.495 & . & 0.979 & 0.371 & 0.544 \\
\hline & $\mathrm{N}$ & 25 & 25 & 25 & 25 & 25 & 25 & 25 & 25 & 25 & 25 & 25 & 25 \\
\hline \multirow{3}{*}{$\begin{array}{l}\text { Total Time } \\
\text { Consumed (min) }\end{array}$} & $\begin{array}{l}\text { Correlation } \\
\text { Coefficient }\end{array}$ & 0.263 & -0.224 & 0.369 & -0.207 & 1 & $.421^{*}$ & $.432^{*}$ & $-.490^{*}$ & . & 0.055 & $.475^{*}$ & -0.203 \\
\hline & $\begin{array}{l}\text { Sig. (2- } \\
\text { tailed) }\end{array}$ & 0.204 & 0.283 & 0.069 & 0.32 & . & 0.036 & 0.031 & 0.013 & . & 0.794 & 0.016 & 0.33 \\
\hline & $\mathrm{N}$ & 25 & 25 & 25 & 25 & 25 & 25 & 25 & 25 & 25 & 25 & 25 & 25 \\
\hline \multirow{3}{*}{$\begin{array}{l}\text { Total No. of } \\
\text { Clicks }\end{array}$} & $\begin{array}{l}\text { Correlation } \\
\text { Coefficient }\end{array}$ & 0.201 & -0.185 & 0.103 & 0.184 & $.421^{*}$ & 1 & $.860^{* * *}$ & $-.599^{* *}$ & . & 0.123 & 0.12 & -0.169 \\
\hline & $\begin{array}{l}\text { Sig. (2- } \\
\text { tailed) } \\
\end{array}$ & 0.334 & 0.376 & 0.623 & 0.378 & 0.036 & . & 0 & 0.002 & . & 0.558 & 0.568 & 0.419 \\
\hline & $\mathrm{N}$ & 25 & 25 & 25 & 25 & 25 & 25 & 25 & 25 & 25 & 25 & 25 & 25 \\
\hline \multirow{3}{*}{$\begin{array}{l}\text { Avg. Clicks to } \\
\text { Perform a Single } \\
\text { Task }\end{array}$} & $\begin{array}{l}\text { Correlation } \\
\text { Coefficient }\end{array}$ & 0.204 & -0.098 & 0.181 & 0.256 & $.432^{*}$ & $.860^{* *}$ & 1 & $-.838^{* *}$ & . & 0.185 & 0.185 & -0.225 \\
\hline & $\begin{array}{l}\text { Sig. (2- } \\
\text { tailed) }\end{array}$ & 0.327 & 0.64 & 0.388 & 0.216 & 0.031 & 0 & . & 0 & . & 0.376 & 0.377 & 0.279 \\
\hline & $\mathrm{N}$ & 25 & 25 & 25 & 25 & 25 & 25 & 25 & 25 & 25 & 25 & 25 & 25 \\
\hline \multirow{3}{*}{$\begin{array}{l}\text { No of Tasks } \\
\text { Performed }\end{array}$} & $\begin{array}{l}\text { Correlation } \\
\text { Coefficient }\end{array}$ & -0.154 & 0.128 & -0.217 & -0.143 & $-.490^{*}$ & $\begin{array}{l}.599^{* *} \\
\end{array}$ & $-.838^{* *}$ & 1 & . & -0.196 & -0.297 & 0.226 \\
\hline & $\begin{array}{l}\text { Sig. (2- } \\
\text { tailed) }\end{array}$ & 0.463 & 0.542 & 0.297 & 0.495 & 0.013 & 0.002 & 0 & . & . & 0.348 & 0.149 & 0.277 \\
\hline & $\mathrm{N}$ & 25 & 25 & 25 & 25 & 25 & 25 & 25 & 25 & 25 & 25 & 25 & 25 \\
\hline Learnability & $\begin{array}{l}\text { Correlation } \\
\text { Coefficient }\end{array}$ & & & & & & & & . & . & . & . & . \\
\hline
\end{tabular}




\begin{tabular}{|c|c|c|c|c|c|c|c|c|c|c|c|c|c|}
\hline & $\begin{array}{l}\begin{array}{l}\text { Sig. (2- } \\
\text { tailed) }\end{array} \\
\end{array}$ & . & & & & . & . & . & & . & . & . & . \\
\hline & $\mathrm{N}$ & 25 & 25 & 25 & 25 & 25 & 25 & 25 & 25 & 25 & 25 & 25 & 25 \\
\hline \multirow{3}{*}{$\begin{array}{l}\text { Content } \\
\text { Relevance }\end{array}$} & $\begin{array}{l}\text { Correlation } \\
\text { Coefficient }\end{array}$ & -0.33 & -0.368 & $.445^{*}$ & 0.006 & 0.055 & 0.123 & 0.185 & -0.196 & . & 1 & 0.011 & -0.252 \\
\hline & $\begin{array}{l}\text { Sig. (2- } \\
\text { tailed) } \\
\end{array}$ & 0.107 & 0.07 & 0.026 & 0.979 & 0.794 & 0.558 & 0.376 & 0.348 & . & . & 0.957 & 0.225 \\
\hline & $\mathrm{N}$ & 25 & 25 & 25 & 25 & 25 & 25 & 25 & 25 & 25 & 25 & 25 & 25 \\
\hline \multirow{3}{*}{ Communicative } & $\begin{array}{l}\text { Correlation } \\
\text { Coefficient } \\
\end{array}$ & 0.025 & -0.326 & 0.083 & -0.187 & $.475^{*}$ & 0.12 & 0.185 & -0.297 & . & 0.011 & 1 & -0.128 \\
\hline & \begin{tabular}{|l}
$\begin{array}{l}\text { Sig. (2- } \\
\text { tailed) }\end{array}$ \\
\end{tabular} & 0.905 & 0.112 & 0.693 & 0.371 & 0.016 & 0.568 & 0.377 & 0.149 & . & 0.957 & . & 0.541 \\
\hline & $\mathrm{N}$ & 25 & 25 & 25 & 25 & 25 & 25 & 25 & 25 & 25 & 25 & 25 & 25 \\
\hline
\end{tabular}

*. Correlation is significant at the 0.05 level (2-tailed).

**. Correlation is significant at the 0.01 level (2-tailed). 


\section{APPENDIX 5 Two-Way ANOVA}

Table I: Table Showing Mean, Standard Deviation for the Variable of User Performance: Avg. Clicks to Perform a Single Task

\begin{tabular}{|l|l|l|l|l|}
\hline \multirow{2}{*}{ Group of User } & \multirow{2}{*}{} & \multirow{2}{*}{ 95\% Confidence Interval } \\
\cline { 5 - 5 } & Mean & Std. Error & Lower Bound & Upper Bound \\
\hline Urban Planning & 15.740 & 2.586 & 10.258 & 21.222 \\
Environmental Engineering & 14.688 & 3.233 & 7.835 & 21.540 \\
GIS & 7.250 & 3.233 & .397 & 14.103 \\
Management Studies & 15.942 & 2.639 & 10.346 & 21.537 \\
English Literature & 19.150 & 3.233 & 12.297 & 26.003 \\
\hline
\end{tabular}

Based on modified population marginal mean.

\section{Estimated Marginal Means of Avg Clicks to perform a single task}

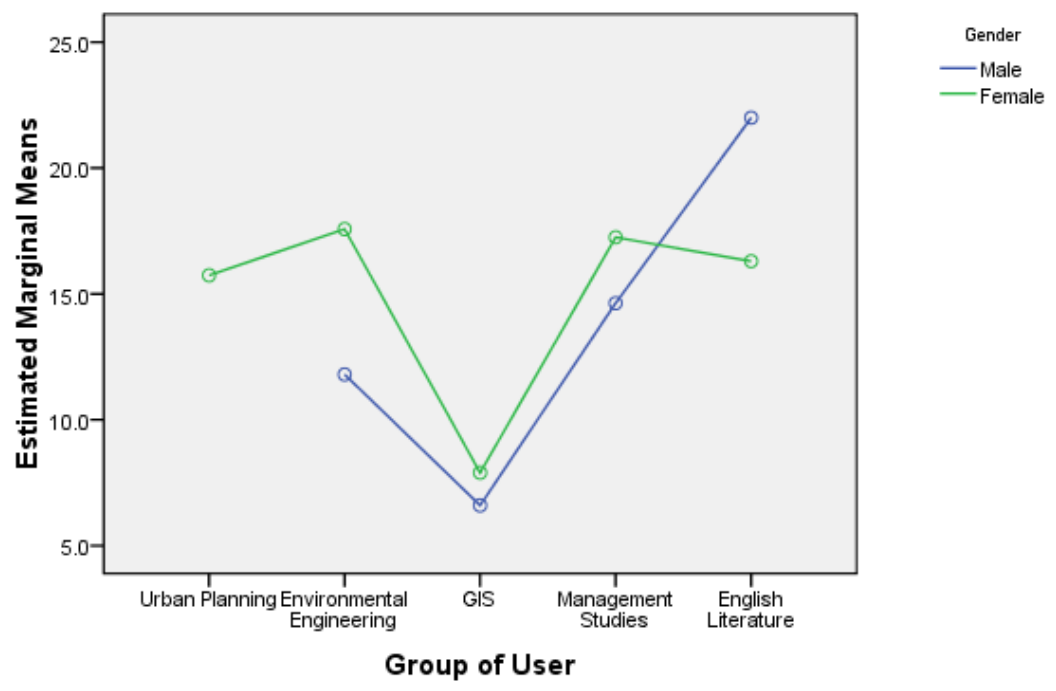

Non-estimable means are not plotted

Table II: User Group-wise Comparison, Mean Difference and Significance for the Performance Variable: Average Clicks to Perform a Single Task

Tukey HSD

\begin{tabular}{|c|c|c|c|c|c|c|}
\hline \multirow{2}{*}{$\begin{array}{l}\text { (I) Group of } \\
\text { User }\end{array}$} & \multirow[b]{2}{*}{ (J) Group of User } & \multirow{2}{*}{$\begin{array}{l}\text { Mean Difference } \\
(\mathrm{I}-\mathrm{J})\end{array}$} & \multirow[b]{2}{*}{ Std. Error } & \multirow[b]{2}{*}{ Sig. } & \multicolumn{2}{|c|}{$95 \%$ Confidence Interval } \\
\hline & & & & & Lower Bound & Upper Bound \\
\hline \multirow{3}{*}{$\begin{array}{l}\text { Urban } \\
\text { Planning }\end{array}$} & Environmental Engineering & -.680 & 3.6573 & 1.000 & -11.885 & 10.525 \\
\hline & GIS & 8.100 & 3.6573 & .224 & -3.105 & 19.305 \\
\hline & Management Studies & .060 & 3.6573 & 1.000 & -11.145 & 11.265 \\
\hline
\end{tabular}




\begin{tabular}{|ll|l|l|l|l|l|}
\hline \multicolumn{2}{|c|}{ English Literature } & -5.120 & 3.6573 & .636 & -16.325 & 6.085 \\
\hline Environmental & Urban Planning & .680 & 3.6573 & 1.000 & -10.525 & 11.885 \\
& GIS & 8.780 & 3.6573 & .165 & -2.425 & 19.985 \\
& Management Studies & .740 & 3.6573 & 1.000 & -10.465 & 11.945 \\
& English Literature & -4.440 & 3.6573 & .744 & -15.645 & 6.765 \\
\hline GIS & Urban Planning & -8.100 & 3.6573 & .224 & -19.305 & 3.105 \\
& Environmental Engineering & -8.780 & 3.6573 & .165 & -19.985 & 2.425 \\
& Management Studies & -8.040 & 3.6573 & .230 & -19.245 & 3.165 \\
& English Literature & $-13.220^{*}$ & 3.6573 & .017 & -24.425 & -2.015 \\
\hline $\begin{array}{l}\text { Management } \\
\text { Studies }\end{array}$ & Urban Planning & -.060 & 3.6573 & 1.000 & -11.265 & 11.145 \\
& Environmental Engineering & -.740 & 3.6573 & 1.000 & -11.945 & 10.465 \\
& GIS & 8.040 & 3.6573 & .230 & -3.165 & 19.245 \\
& English Literature & -5.180 & 3.6573 & .627 & -16.385 & 6.025 \\
\hline English & Urban Planning & 5.120 & 3.6573 & .636 & -6.085 & 16.325 \\
Literature & Environmental Engineering & 4.440 & 3.6573 & .744 & -6.765 & 15.645 \\
& GIS & $13.220^{*}$ & 3.6573 & .017 & 2.015 & 24.425 \\
& Management Studies & 5.180 & 3.6573 & .627 & -6.025 & 16.385 \\
\hline
\end{tabular}

Based on observed means.

The error term is Mean Square (Error) =

33.439

*. The mean difference is significant at the .05 level.

Table III: User Group-wise Mean and Significance for the Performance Variable: Average Clicks to Perform a Single Task

Tukey HSD

\begin{tabular}{|l|l|l|l|}
\hline \multirow{2}{*}{ Group of User } & \multirow{2}{*}{ N } & \multicolumn{2}{|l|}{ Subset } \\
\cline { 3 - 4 } & $\mathrm{N}$ & 1 & 2 \\
\hline GIS & 5 & 7.640 & \\
Management Studies & 5 & 15.680 & 15.680 \\
Urban Planning & 5 & 15.740 & 15.740 \\
Environmental Engineering & 5 & 16.420 & 16.420 \\
English Literature & 5 & & 20.860 \\
Sig. & & .165 & .627 \\
\hline
\end{tabular}

Means for groups in homogeneous subsets are displayed.

Based on observed means.

The error term is Mean Square (Error) $=33.439$. 


\section{APPENDIX 6 Sequence Diagrams of Co-PPGIS Services}

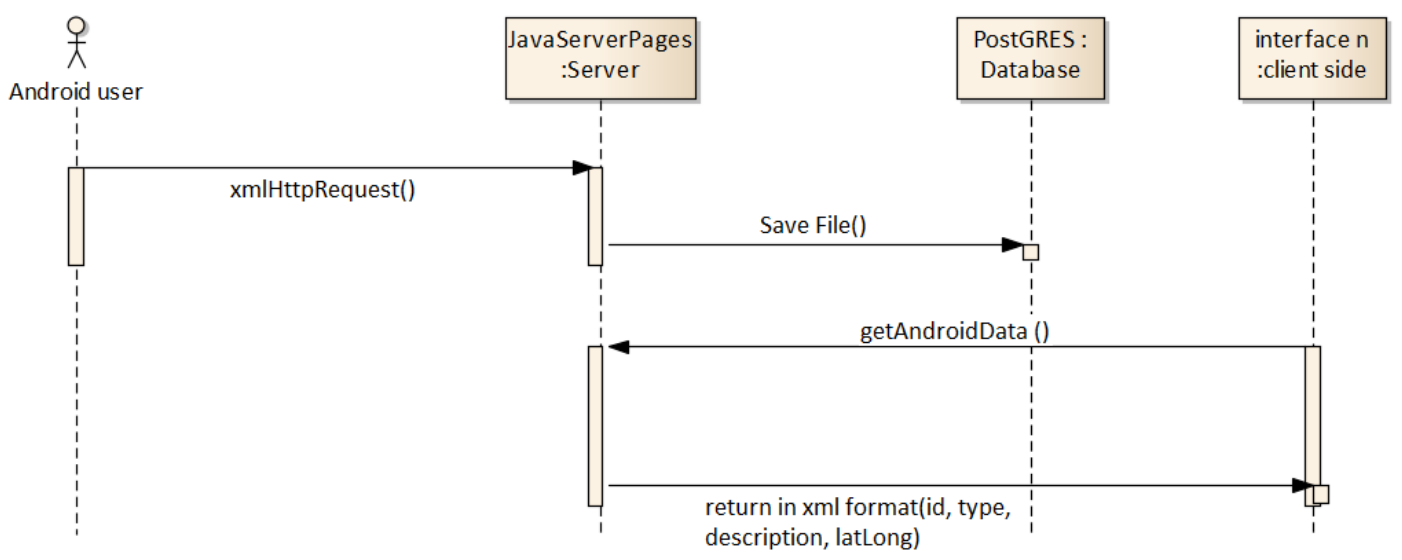

Figure I: Android Service

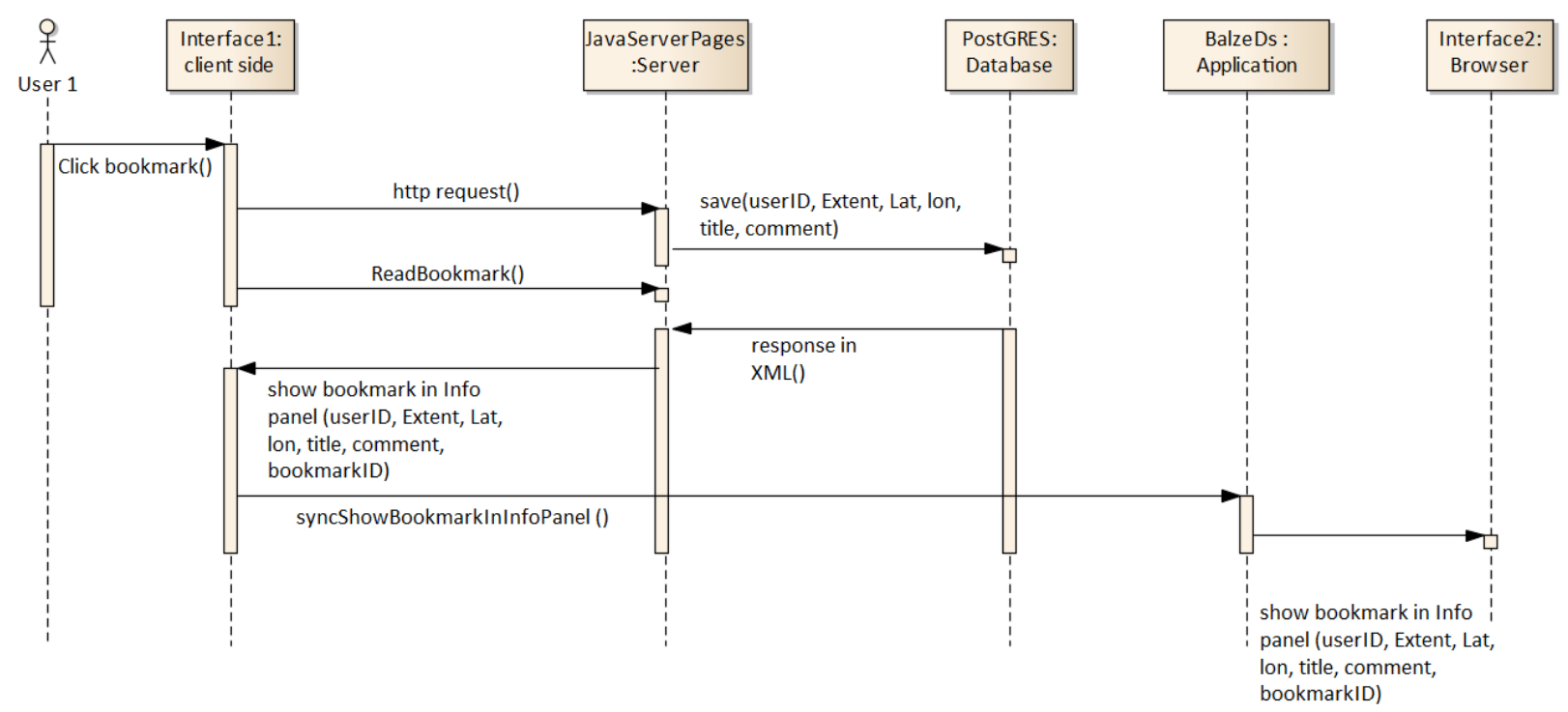

Figure II: Bookmark Service

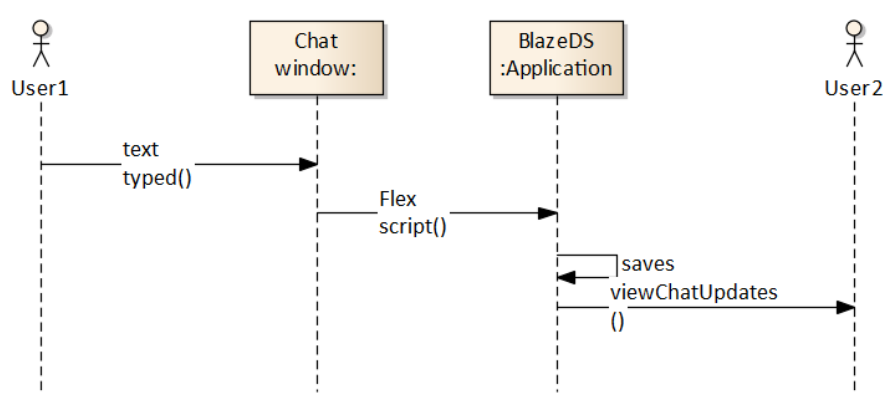

Figure III: Chat Service 


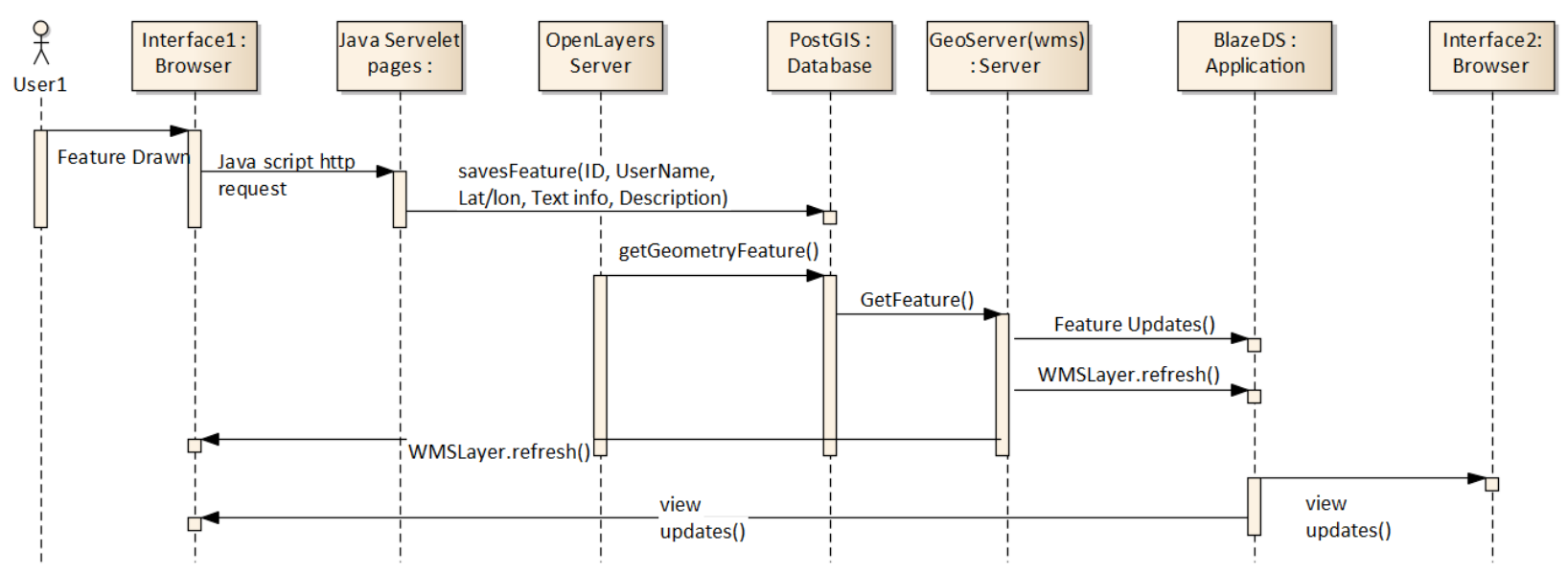

Figure IV: Editing Toolbar Service

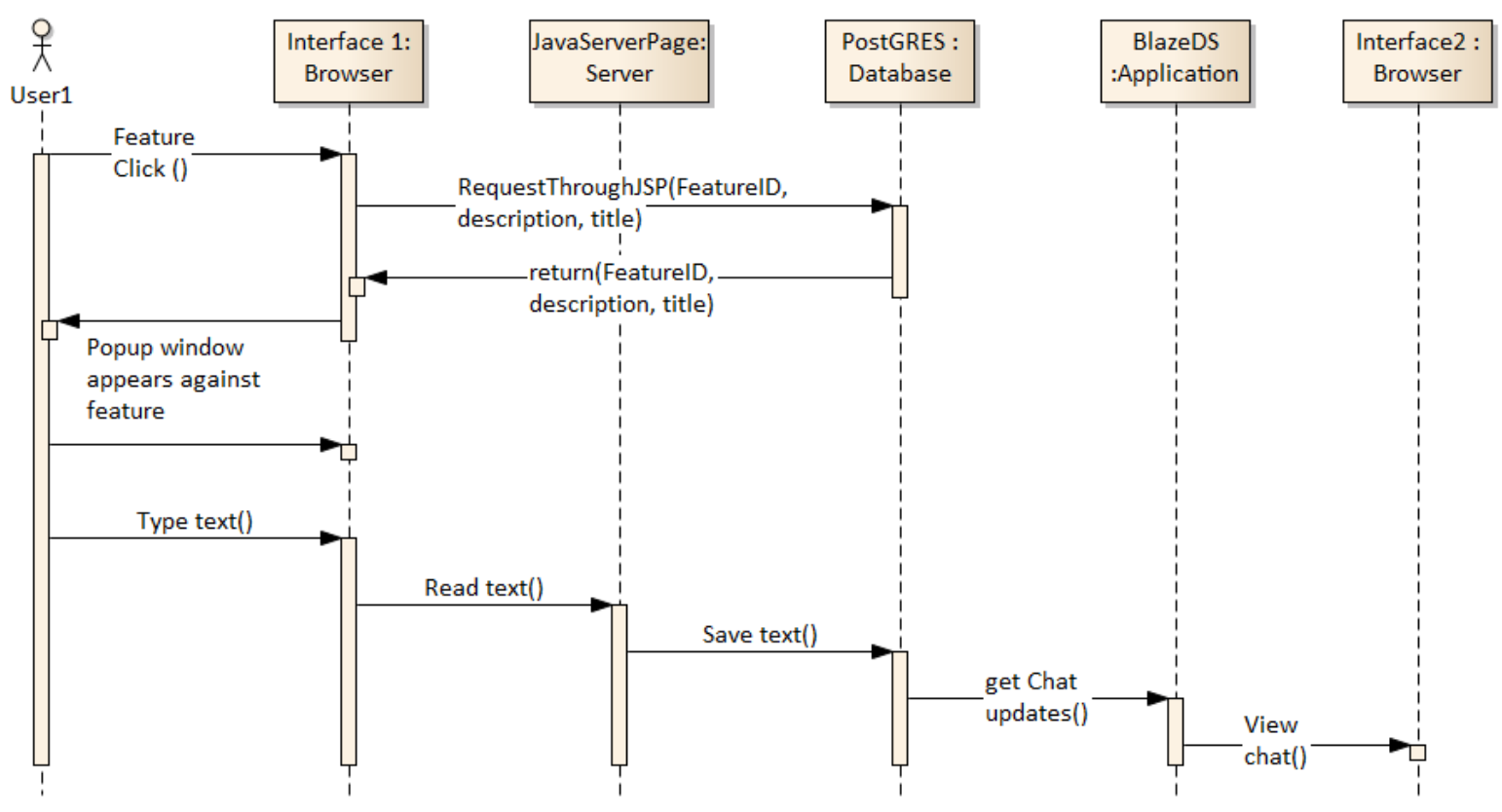

Figure V: Feature-based Chat Service 


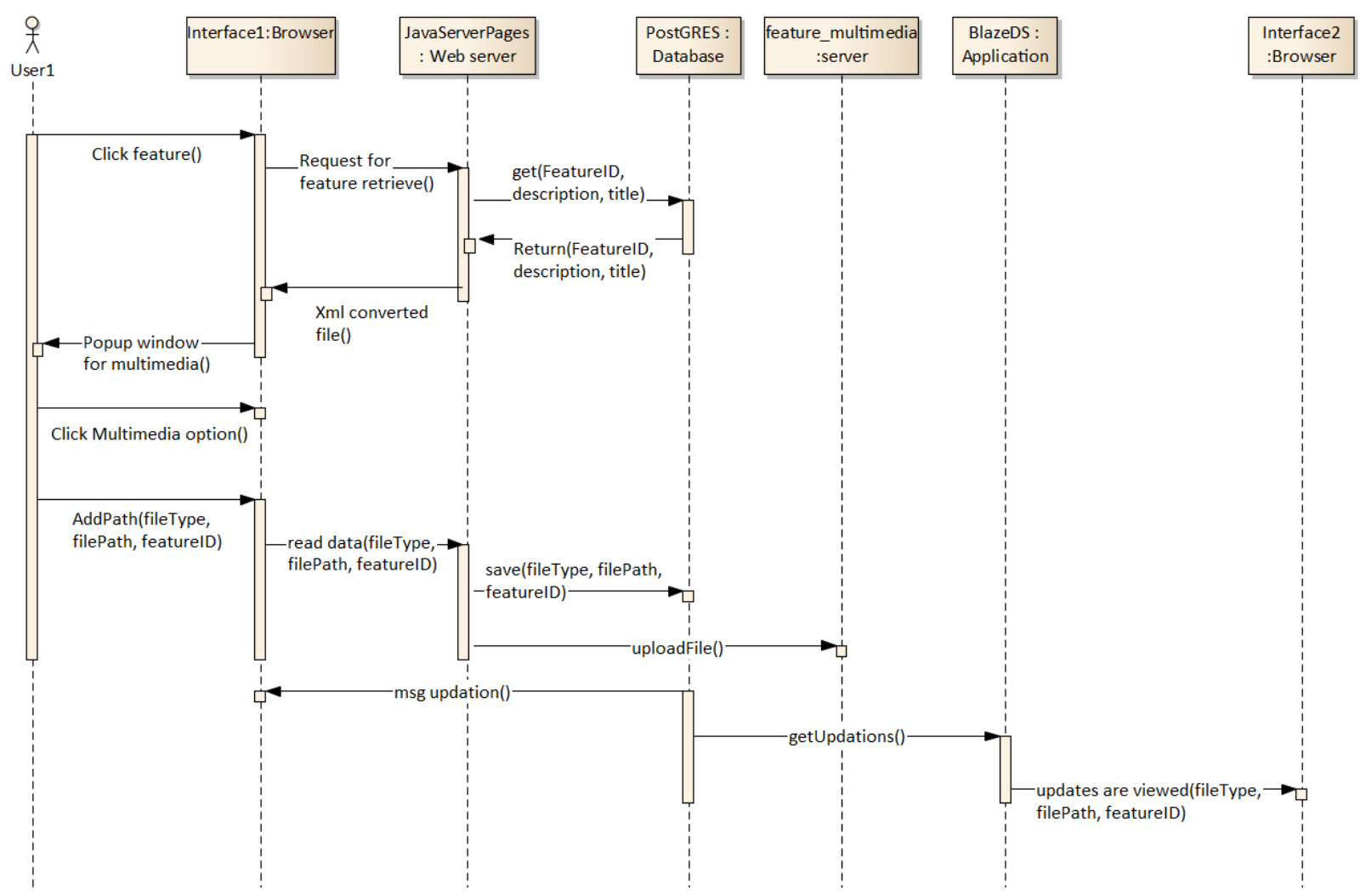

Figure VI: Feature-based Multimedia Service

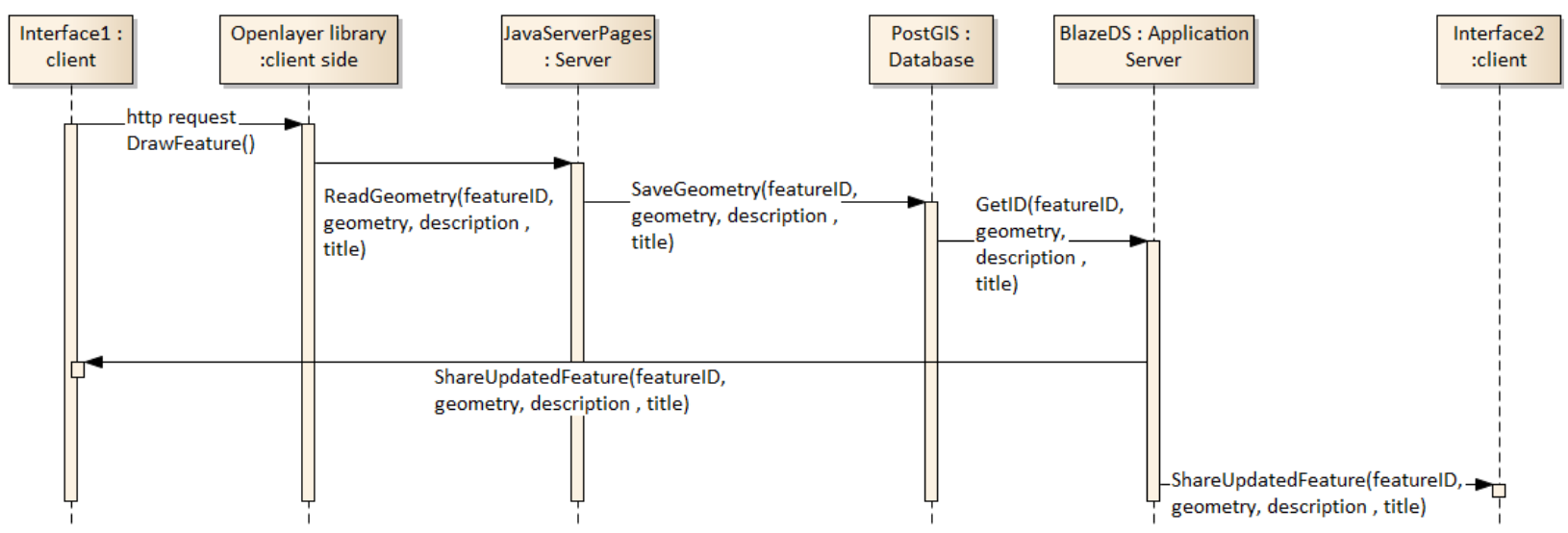

Figure VII: Geometry Feature Service 


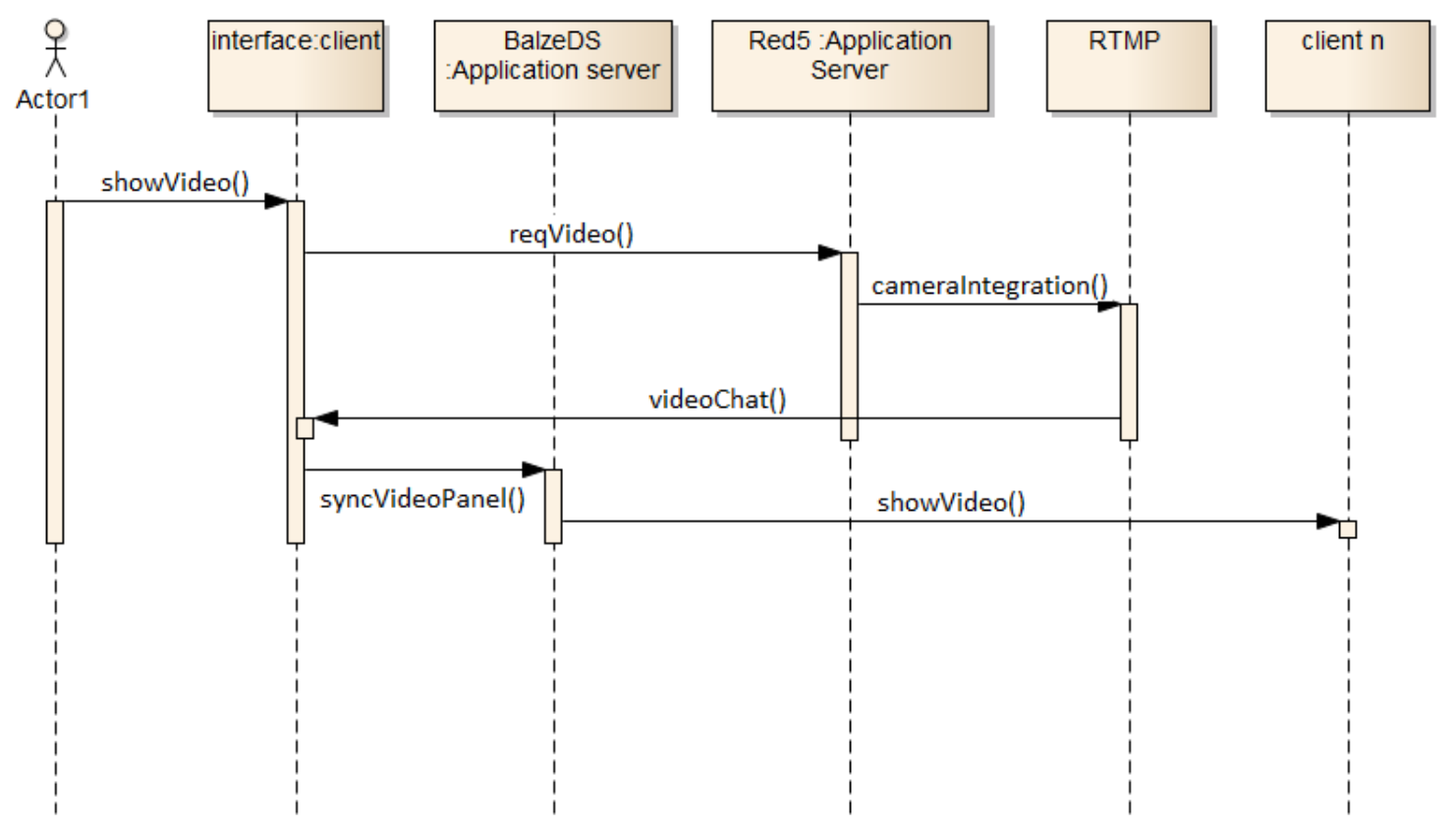

Figure VIII: Live Video Streaming Service

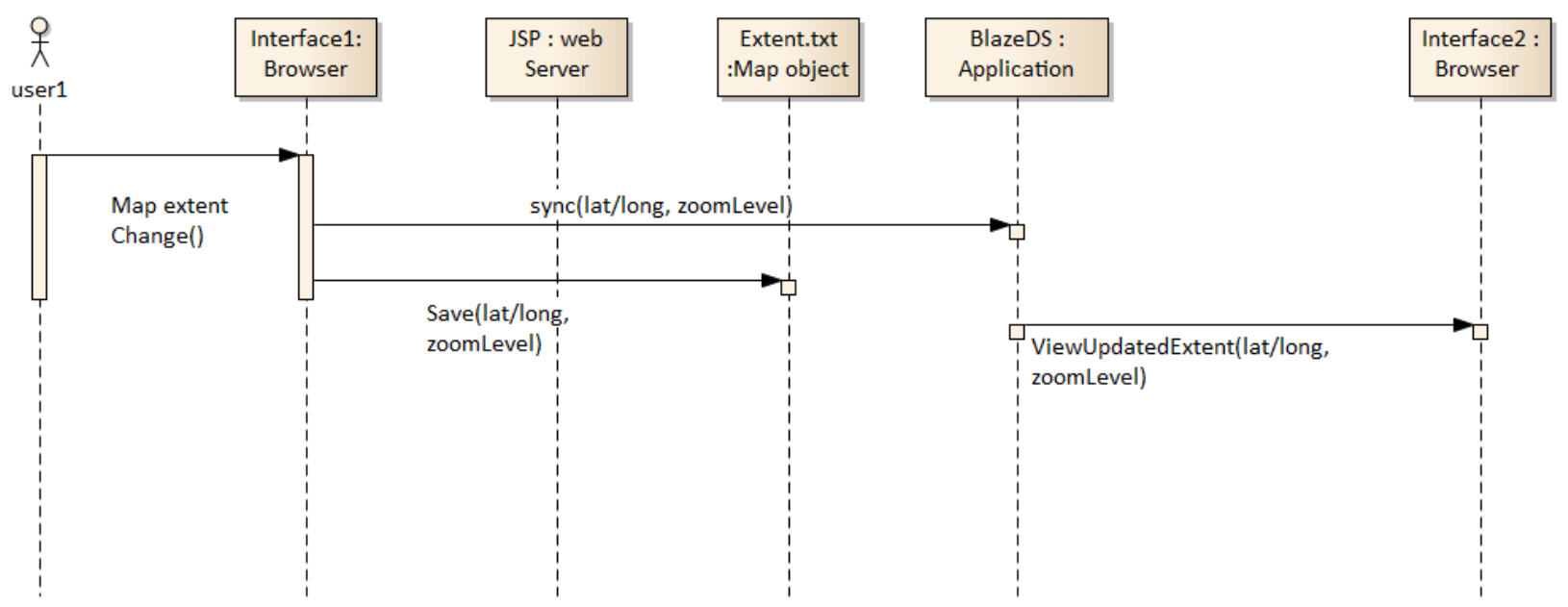

Figure IX: Map-sharing Service 


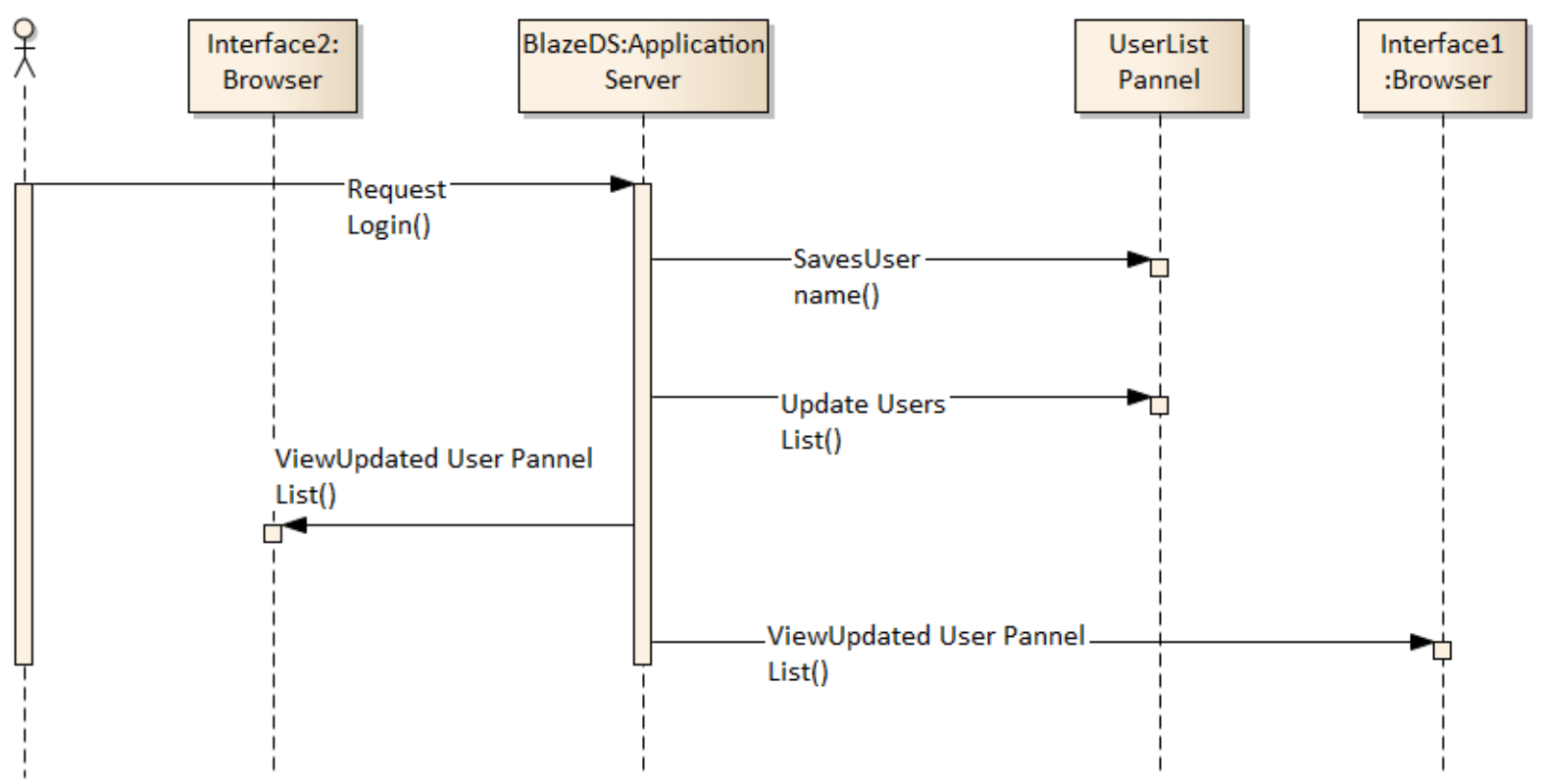

Figure X: Member List Service

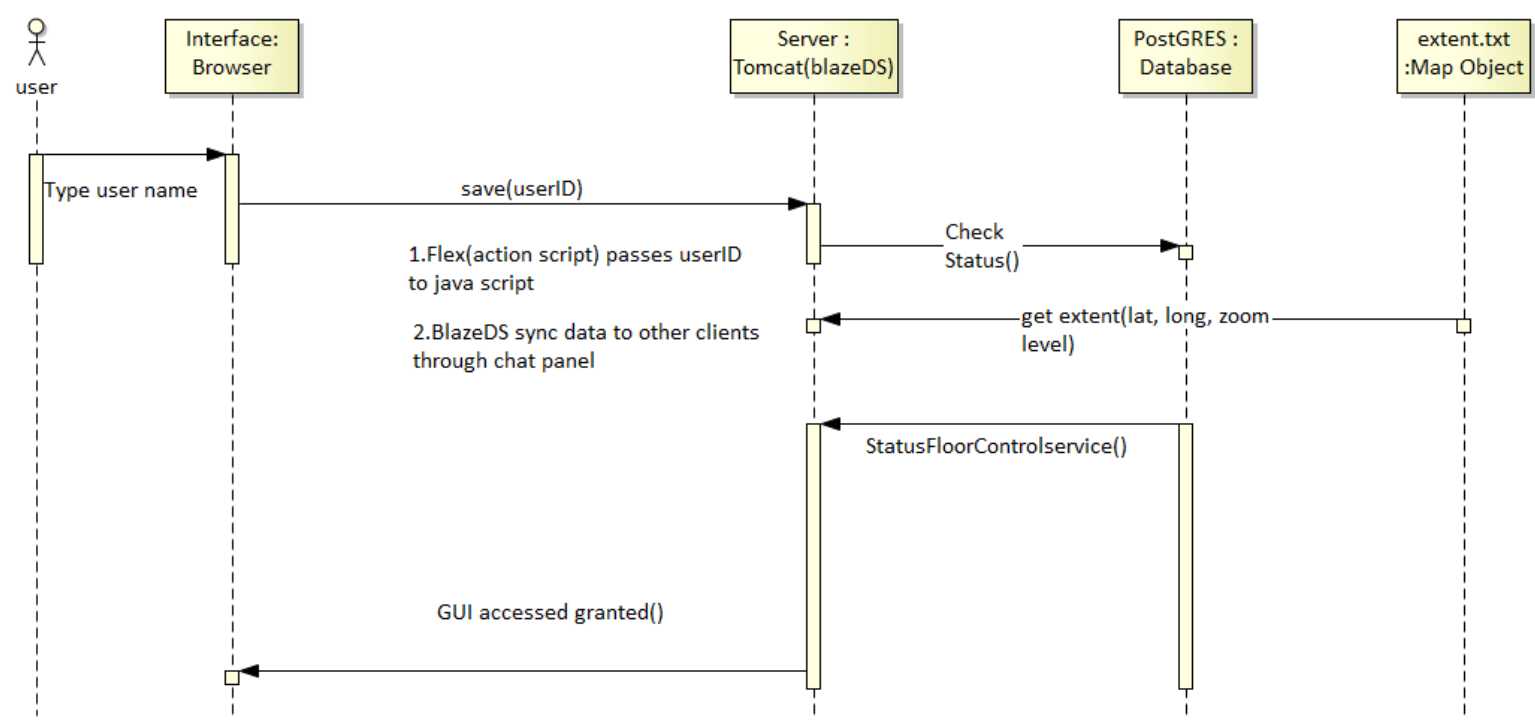

Figure XI: Entering in Common Conference Room 


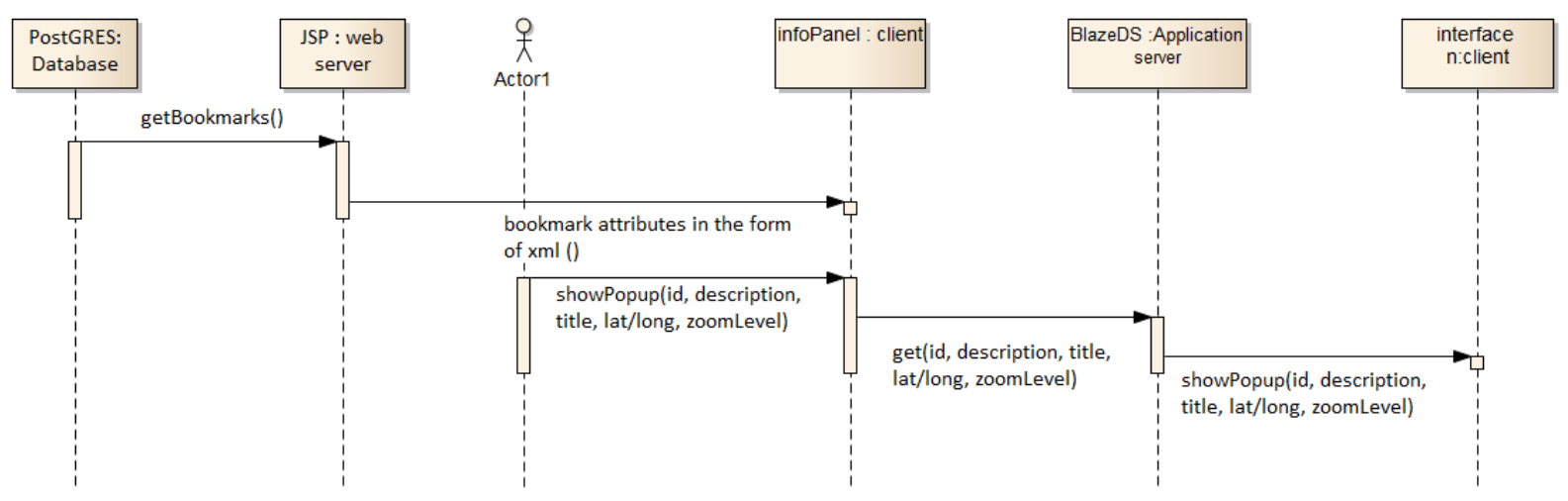

Figure XII: Identify Feature Popup Service

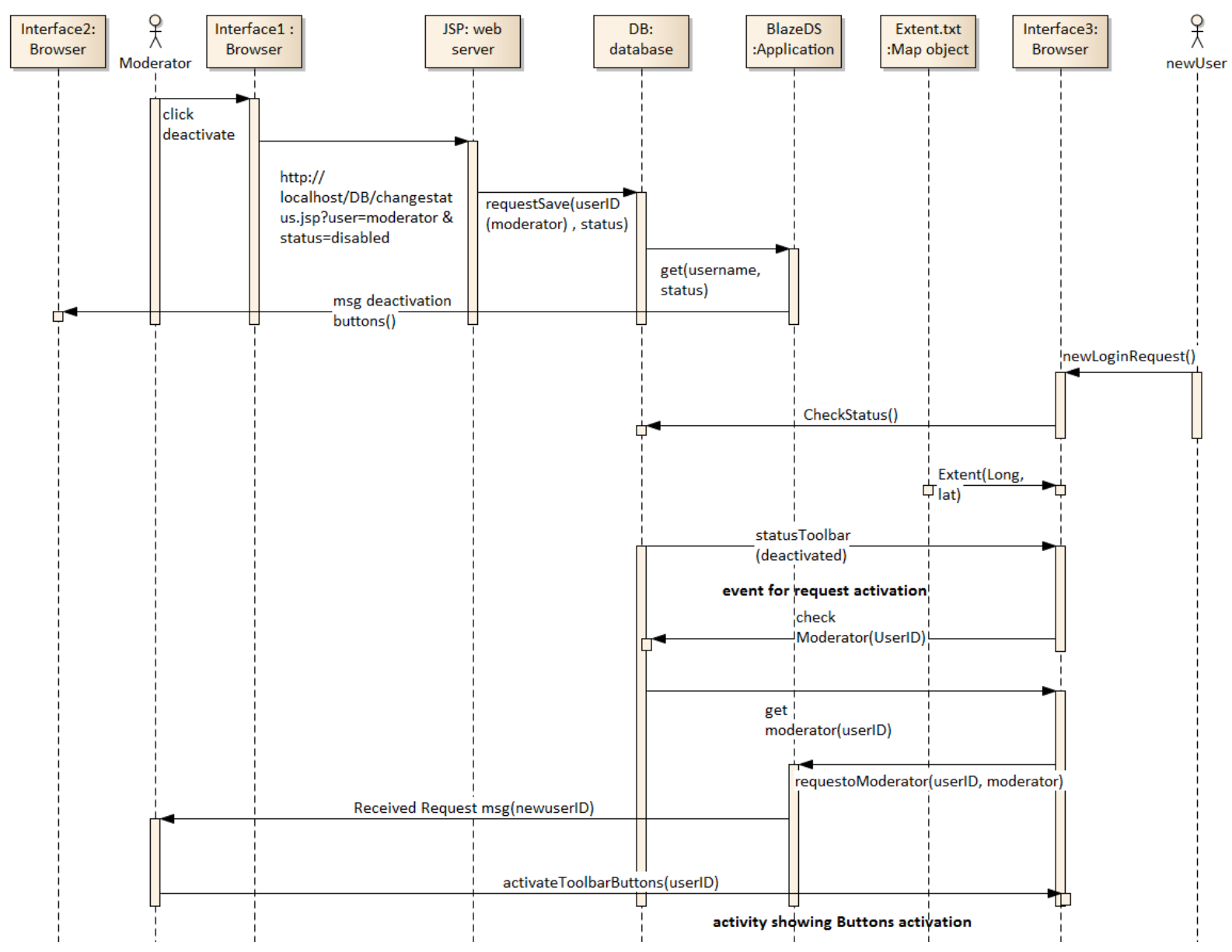

Figure XIII: Floor Control Service 


\title{
APPENDIX 7 Usability Aspects to Measure Social Adoptability of Co-PPGIS
}

\author{
Table I: Usability Aspects to Measure Social Adoptability of Co-PPGIS \\ Social Adoptability of Co-PPGIS \\ Usability Aspects/ \\ Benchmarks \\ Learnability

\section{Co-PPGIS Measuring Aspects/ Measurement of the Benchmark} \\ Task-based scenarios are provided to the new users during the hands-on \\ practice exercise and no immediate assistance is provided. By all means, users \\ have to learn by themselves. Therefore, the time spent on reading and/or \\ exploration the prototype collaborative functions (about how to use the $\mathrm{CO}$ - \\ PPGIS to participate) is used to represent learnability rate. Some of the \\ ergonomics design considerations, in relation to learnability aspect are \\ incorporated during the design and development of the prototype: 1) how easy is \\ it for new users to learn to perform the specific task? and 2)for more complicated \\ tasks, are there sufficient help features such as tutorials, in-line tips and hints, \\ tool tips, etc.? \\ How easy is it to understand and recognize the usefulness of the prototype or \\ the tools in it" (ISO 1998)? It is quantified by the time to learn, e.g., how quickly \\ does a user learn to communicate using the prototype's audio/video component \\ during participatory planning practices?
}

Intuitiveness

Task Efficiency

Preciseness

Fault Tolerance

Affordance

Memorability

Likeability
How obvious and easy is for the user to perform the task in order to accomplish?

Efficiency refers to the system's ability to fulfill its functions and objectives while taking a minimal amount of resources, so that once the user has learned the system, a higher level of productivity is possible (ISO 1998; Zhao 2007). It is calculated by the time to achieve a pre-specified task on first attempt by the participant. Tasks efficiency level can be measured by answering the questions, for example: 1) are users performing tasks optimally? and 2) are there ways to streamline and reduce the time it takes to complete the task?

Preciseness is related to the systems' errors identified and faced to the participants while performing the scenario-based tasks during exploration of the prototype functionalities. This evaluating aspect is helpful in identifying 1) how prone to errors is the task? 2) what are the reasons for any error?, and 3) how can we improve the interface to lower the errors and unneeded repetition?

It is related to users' errors committed while performing the scenario-based testing, e.g., if a user makes a mistake while performing the task, how fast can they recover?

Are interactive elements (such as dropdown menus, buttons, and links and input text boxes) related to the accomplishment of a task interactive and within convenient reach? Is it evident what the results of a user action will be when the user decides to interact with it by clicking, mouse hovering, etc.?

It is related to memorability of the interface, e.g., how easy is for the user to repeat the specific task?

It is related to prototype adoptability, e.g., How easily do the intended users accomplish the system's functionalities to make it adoptable? Goal of the likeability aspect is to provide an adoptable interface that provides a virtual GISbased platform for participation to the users (GIS/skilled professionals, citizens and non-professional) in the ongoing discussion of spatially-related issues. 

achieve their goals" (ISO 1998). It is measured by number of power tasks (e.g., exploring map-sharing function or making annotation on the map) completed successfully on first attempt without committing any mistake. How many less number of unproductive navigation and searches do users perform?

Table II: Usability Aspects to Measure Practical Adoptability of Co-PPGIS

Practical Adoptability of Co-PPGIS

Usability Benchmarks/Sub Categories Aspects

HCI

Efficiency/

Response

time$$
\begin{aligned}
& \text { i) } \begin{array}{l}
\text { Human Computer } \\
\text { Interaction (HCI) } \\
\text { Rules }
\end{array} \\
& \text { i) }
\end{aligned}
$$

i) Findability

ii) Response time

iii) Information

Architecture

iv) Efficiency-of Navigation

Less Error

i) Code Quality

Flexibility

Readability

i) Cross-

ii) Colour Choice

i) Ease-of

\section{Co-PPGIS Measuring Aspects/ Measurement} of the Benchmark

Is the system designed by adopting $\mathrm{HCI}$ rules, i.e., strive for consistency, offer informative feedback and error prevention?

Are there adequate features such as search boxes, links and navigation menus that aid in finding relevant webpages?

How fast does it take to load the entire webpage? The contents/elements of the prototype interface should not be heavy.

How well are the pages of prototype Web interface categorized and organized? How well are navigational features (menus) constructed?

How many number of clicks or keyboard stroke does it take to get the user to the page of interest?

Does the prototype Web interface design use web development best practices (ISO and IEEE standards, etc.) to maintain code quality in order to produce error less results? browser/Platform

Does the prototype Web interface work in as many browsers (Firefox, Chrome, etc.) as possible? Is the site responsive, flexibly changing the Web layout depending on screen pixel sizes, display resolution and/or how does the user view it?

Are the colours used during the prototype Web interface designing high contrast? Do these colours create any difficulty to people with the disability of colorblindness or poor vision? Comprehensiveness

Do the textual contents of prototype interface easy to understand by the attended user? Are the words being used familiar to the average attended user (public participant) or are they too complex and uncommon? Are sentences and paragraphs as comprehensive as possible? Is the information accurate, of high quality and well-written?

ii) Legibility
Are the fonts (of textual information) of prototype interface big enough? Is there enough contrast between the text and its background? Do 


\begin{tabular}{|c|c|c|c|}
\hline & & & $\begin{array}{l}\text { font characteristics such as size, spacing and } \\
\text { color make reading longer passages easy or do } \\
\text { they strain the eyes? }\end{array}$ \\
\hline Helpfulness & i) & $\begin{array}{l}\text { HTML Accessibility } \\
\text { Features }\end{array}$ & $\begin{array}{l}\text { There are HTML features and techniques } \\
\text { that aid users with visual impairments. Are these } \\
\text { features and techniques being used? }\end{array}$ \\
\hline \multirow[t]{4}{*}{ Satisfaction } & i) & Fulfillment & $\begin{array}{l}\text { Do participants feel satisfied after } \\
\text { interacting with the CO-PPGIS? This is reflected } \\
\text { in the levels of speed, completeness and } \\
\text { correctness in the user performance during the } \\
\text { testing. It is a feedback of users after finishing } \\
\text { interaction with the system. In the polling and } \\
\text { feedback section, the users were asked to rate } \\
\text { their overall experience with CO-PPGIS on a } \\
\text { five point scale ranging from } 1 \text { to } 5 \text {. }\end{array}$ \\
\hline & ii) & Usefulness & $\begin{array}{l}\text { Does the public participant get worthy } \\
\text { feeling after using the prototype? Does the } \\
\text { prototype provide users an easy access to } \\
\text { additional information in the form of projects } \\
\text { documentation, video, pictures, etc., to contribute } \\
\text { in the municipal planning related workflow for } \\
\text { better and collaborative decision-making? What } \\
\text { are the limitations in terms of user friendliness } \\
\text { for functional aspects of the prototype? }\end{array}$ \\
\hline & iii) & Enjoyment & $\begin{array}{l}\text { Is the experience of being exploring the } \\
\text { prototype functionalities fun and positive } \\
\text { emotions not troublesome? Do users appreciate } \\
\text { and enjoy the prototype while participating on } \\
\text { the map and/or exploring collaboratively spatial } \\
\text { contents of municipality project related data? }\end{array}$ \\
\hline & iv) & Positive Response & $\begin{array}{l}\text { It refers to "freedom from discomfort and } \\
\text { positive attitudes to the use of the product" (ISO } \\
\text { 1998). Do users feel pleased, enthusiastic, } \\
\text { satisfied, etc., when they interact with the } \\
\text { prototype? }\end{array}$ \\
\hline Initial Cost & i) & Cost Effectiveness & $\begin{array}{l}\text { This factor is related to practical adoptability } \\
\text { of the prototype in relation to its financial } \\
\text { budgeting, e.g., smaller municipalities have less } \\
\text { financial resources and therefore, prefer to } \\
\text { acquire a low cost system. Moreover, the initial } \\
\text { cost refers to end-user investments on } \\
\text { development of prototype, which is almost } \\
\text { absent, because prototype is developed using } \\
\text { open source software, components/modules and } \\
\text { open source GIS technologies. }\end{array}$ \\
\hline
\end{tabular}




\section{APPENDIX 8 List of Selected Application for Evaluation}

\begin{tabular}{|c|c|c|c|}
\hline No & \multicolumn{2}{|c|}{ Application $\quad$ Description } & URL \\
\hline $1 * *$ & $\begin{array}{l}\text { Virtual } \\
\text { Slaithwaite } \\
\text { United Kingdom }\end{array}$ & $\begin{array}{l}\text { The Slaithwaite Public Participation Geographical } \\
\text { Information System is redesigned using JAVA in } \\
\text { 2003. This system was designed to accumulate } \\
\text { citizens' ideas and comments. The users had the } \\
\text { window (of opportunity) to write their ideas as well } \\
\text { as thoughts on any point or location on the map } \\
\text { using add text tool. These comments were } \\
\text { represented with small dots (flags) and can be } \\
\text { viewed by any user for participation (Evans et al. } \\
\text { 1999). The user can use some other GIS mapping } \\
\text { tools' functionalities, for example, map navigation } \\
\text { can be performed with zoom and pan tools. }\end{array}$ & $\begin{array}{l}\text { http://www.ccg.leeds.a } \\
\text { c.uk/slaithwaite/ }\end{array}$ \\
\hline $2 * *$ & $\begin{array}{l}\text { MapChat } \\
\text { (University of } \\
\text { Waterloo, } \\
\text { Canada) }\end{array}$ & $\begin{array}{l}\text { MapChat is a Web-based application that consists of } \\
\text { real-time protocols of sharing maps and imagery } \\
\text { among geographically spread participants and/or } \\
\text { decision makers (originally designed by Hall 2006). } \\
\text { Participants can discuss freely in question and } \\
\text { answer format using its real-time chatting } \\
\text { (geochatting) feature. The intended use of this } \\
\text { PPGIS application was to facilitate the citizens } \\
\text { during municipal planning and management-related } \\
\text { activities. This MapChat application has multi-way } \\
\text { discussion forum support using its real-time chatting } \\
\text { feature. The MapChat was designed using a full open } \\
\text { source geospatial stack merging with the Web } 2.0 \\
\text { technologies for instance, the database PostGIS, the } \\
\text { server GeoServer, the client with OpenLayers and } \\
\text { MapFish/GeoExt which is a toolkit to build } \\
\text { OpenLayers application based on the Ext JS } \\
\text { framework. The system (MapChat) offers access to } \\
\text { several types of property, land use, and other } \\
\text { information. }\end{array}$ & $\begin{array}{l}\text { source URL: } \\
\text { http://www.mapchat.ca }\end{array}$ \\
\hline $3 * *$ & $\begin{array}{l}\text { Interactive } \\
\text { Landscape Plan } \\
\text { Königslutter am } \\
\text { Elm (Lower } \\
\text { Saxony, } \\
\text { Germany) }\end{array}$ & $\begin{array}{l}\text { The interactive landscape planning system } \\
\text { "Königslutter am Elm" is an online public } \\
\text { participation geographic information system } \\
\text { sponsored by the Federal Agency for Nature } \\
\text { Protection (FANP, Lower Saxony, Germany; } \\
\text { http://thuja.land.uni-hannover.de/). This online } \\
\text { PPGIS provides access to the public with several } \\
\text { types of information regarding landscape planning } \\
\text { for the area Königslutter am Elm. It was developed } \\
\text { to complement the traditional public participatory } \\
\text { approach. } \\
\text { The intended use of this PPGIS application was to } \\
\text { facilitate the citizens as well as non-professionals. } \\
\text { Although this GIS-based application has almost the }\end{array}$ & $\begin{array}{l}\text { http://thuja.land.uni- } \\
\text { hannover.de/ } \\
\text { notrently, the demo is } \\
\text { accessed in Dec } 2010\end{array}$ \\
\hline
\end{tabular}




\begin{tabular}{|c|c|c|c|}
\hline & & $\begin{array}{l}\text { same GIS functions as to those of the system } \\
\text { discussed earlier (i.e., INFOMAP etc.), it still has } \\
\text { more creditability due to its cost-effective feature. } \\
\text { This system was designed using open source } \\
\text { software (OOS) technologies. University of } \\
\text { Minnesota (UMN) MapServer generates maps and } \\
\text { simple images on the map layer and MySQL } \\
\text { database server handles textual and spatial data. }\end{array}$ & \\
\hline $4 * *$ & $\begin{array}{l}\text { Argumentation } \\
\text { Map (ArgooMap) }\end{array}$ & $\begin{array}{l}\text { Conceptual model of argumentation model was } \\
\text { introduced by Rinner (1999). A prototype system to } \\
\text { support discussion based on maps. Discussion } \\
\text { contributions are structured to facilitate users } \\
\text { understand the evolution of ideas. Annotations are } \\
\text { geographically referenced and linked to geographic } \\
\text { features. }\end{array}$ & $\begin{array}{l}\text { Downloaded at: } \\
\text { http://sourceforge.net/p } \\
\text { rojects/argoomap/ } \\
\text { Demo is not available } \\
\text { online } \\
\text { http://141.117.104.183/ } \\
\text { argoomap/test/about.ht } \\
\mathrm{m}\end{array}$ \\
\hline $5 * *$ & GeoDF & $\begin{array}{l}\text { The GIS-enabled Online Discussion Forum (GeoDF) } \\
\text { has been introduced by Tang as a prototype system } \\
\text { for spatially-related discussions (Tang 2006). The } \\
\text { software intends to facilitate the general public to } \\
\text { participate in the ongoing discussion of spatially- } \\
\text { related issues. Discussion contributions are } \\
\text { structured to facilitate users understand the evolution } \\
\text { of ideas. They are geographically referenced and } \\
\text { linked to geographic features. }\end{array}$ & $\begin{array}{l}\text { http://www.vrm.ca/doc } \\
\text { uments/ACFAS09_tan } \\
\text { g.pdf } \\
\text { Demo is not available } \\
\text { online; evaluation is } \\
\text { based at available } \\
\text { literature on the Web. }\end{array}$ \\
\hline 6 & GeoLink & $\begin{array}{l}\text { A synchronous approach is developed to support } \\
\text { collaboration among users (Chang 2010); Prototype } \\
\text { was introduced by Chang in } 2005 \text { and redesigned in } \\
2010 \text { with more } 3 \text { d-based functionalities. GeoLink is } \\
\text { a light-weight, object-oriented and extensible } \\
\text { application. This application support two clients } \\
\text { work collaboratively in one machine on real-time } \\
\text { basis. }\end{array}$ & $\begin{array}{l}\text { http://www.simal.ryers } \\
\text { on.ca } \\
\text { Demo is not available } \\
\text { online; evaluation is } \\
\text { based at available } \\
\text { literature on the Web. }\end{array}$ \\
\hline
\end{tabular}




\begin{tabular}{|c|c|c|c|}
\hline 7 & $\begin{array}{l}\text { Shaping Dane's } \\
\text { Future, U.S. }\end{array}$ & $\begin{array}{l}\text { A prototype system that allows users to use the } \\
\text { system to visualize maps of the town of Verona } \\
\text { and/or download GIS data onto participant's } \\
\text { computer to create maps or further analysis of the } \\
\text { spatial data. }\end{array}$ & $\begin{array}{l}\text { http://www.lic.wisc.ed } \\
\text { u/shapingdane/resource } \\
\text { s/resources-home.htm }\end{array}$ \\
\hline 8 & CartoVista & $\begin{array}{l}\text { CartoVista is a flexible architecture that makes data } \\
\text { publishing and management very simple while } \\
\text { allowing you to produce rich mapping applications. }\end{array}$ & $\begin{array}{l}\text { http://www.cartovista.c } \\
\text { om/cartovista3/Montre } \\
\text { al.html }\end{array}$ \\
\hline 9 & GeoNB & $\begin{array}{l}\text { GeoNB is a collaborative project led by Service New } \\
\text { Brunswick's Land Information Secretariat. The } \\
\text { system objective is to facilitate the sharing of } \\
\text { provincial geographic data and services among } \\
\text { government departments, industry, organizations, } \\
\text { academia, and the general public. }\end{array}$ & $\begin{array}{l}\text { http://www.snb.ca/geo } \\
\text { nb2/index.html }\end{array}$ \\
\hline 10 & $\begin{array}{l}\text { INFOMAP } \\
\text { Orange County } \\
\text { Interactive } \\
\text { Mapping }\end{array}$ & $\begin{array}{l}\text { The system (InfoMap) offers access to several types } \\
\text { of property, land use, and other information for } \\
\text { Orange County in Florida, USA (Steinmann et al. } \\
\text { 2004). The intended use of this PPGIS application } \\
\text { was to facilitate the citizens. The system was } \\
\text { developed as PPGIS to identify the citizens' views or } \\
\text { comments regarding their county. In this way, } \\
\text { municipal government got a brief idea of their } \\
\text { citizens' concerns (i.e., future coming plans related } \\
\text { to municipal planning and development). }\end{array}$ & $\begin{array}{l}\text { WNAP-MP } \\
\text { http://ocgis1.ocfl.net/G } \\
\text { eocortex/Essentials/We } \\
\text { b/Viewer.aspx?Site=In } \\
\text { fomapPublic }\end{array}$ \\
\hline
\end{tabular}

**Applications (1-5) were selected and considered for the evaluation purposes that meet the selection criteria adopted by the author 